\title{
Renewable cyclic monomers for high-performance biobased polyesters
}

Citation for published version (APA):

Noordzij, G. J. (2020). Renewable cyclic monomers for high-performance biobased polyesters. [Doctoral Thesis, Maastricht University]. Maastricht University. https://doi.org/10.26481/dis.20200925gn

Document status and date:

Published: 01/01/2020

DOI:

10.26481/dis.20200925gn

Document Version:

Publisher's PDF, also known as Version of record

\section{Please check the document version of this publication:}

- A submitted manuscript is the version of the article upon submission and before peer-review. There can be important differences between the submitted version and the official published version of record.

People interested in the research are advised to contact the author for the final version of the publication, or visit the DOI to the publisher's website.

- The final author version and the galley proof are versions of the publication after peer review.

- The final published version features the final layout of the paper including the volume, issue and page numbers.

Link to publication

\footnotetext{
General rights rights.

- You may freely distribute the URL identifying the publication in the public portal. please follow below link for the End User Agreement:

www.umlib.nl/taverne-license

Take down policy

If you believe that this document breaches copyright please contact us at:

repository@maastrichtuniversity.nl

providing details and we will investigate your claim.
}

Copyright and moral rights for the publications made accessible in the public portal are retained by the authors and/or other copyright owners and it is a condition of accessing publications that users recognise and abide by the legal requirements associated with these

- Users may download and print one copy of any publication from the public portal for the purpose of private study or research.

- You may not further distribute the material or use it for any profit-making activity or commercial gain

If the publication is distributed under the terms of Article $25 \mathrm{fa}$ of the Dutch Copyright Act, indicated by the "Taverne" license above, 
Renewable cyclic monomers

for high-performance

biobased polyesters

Geert J. Noordzij

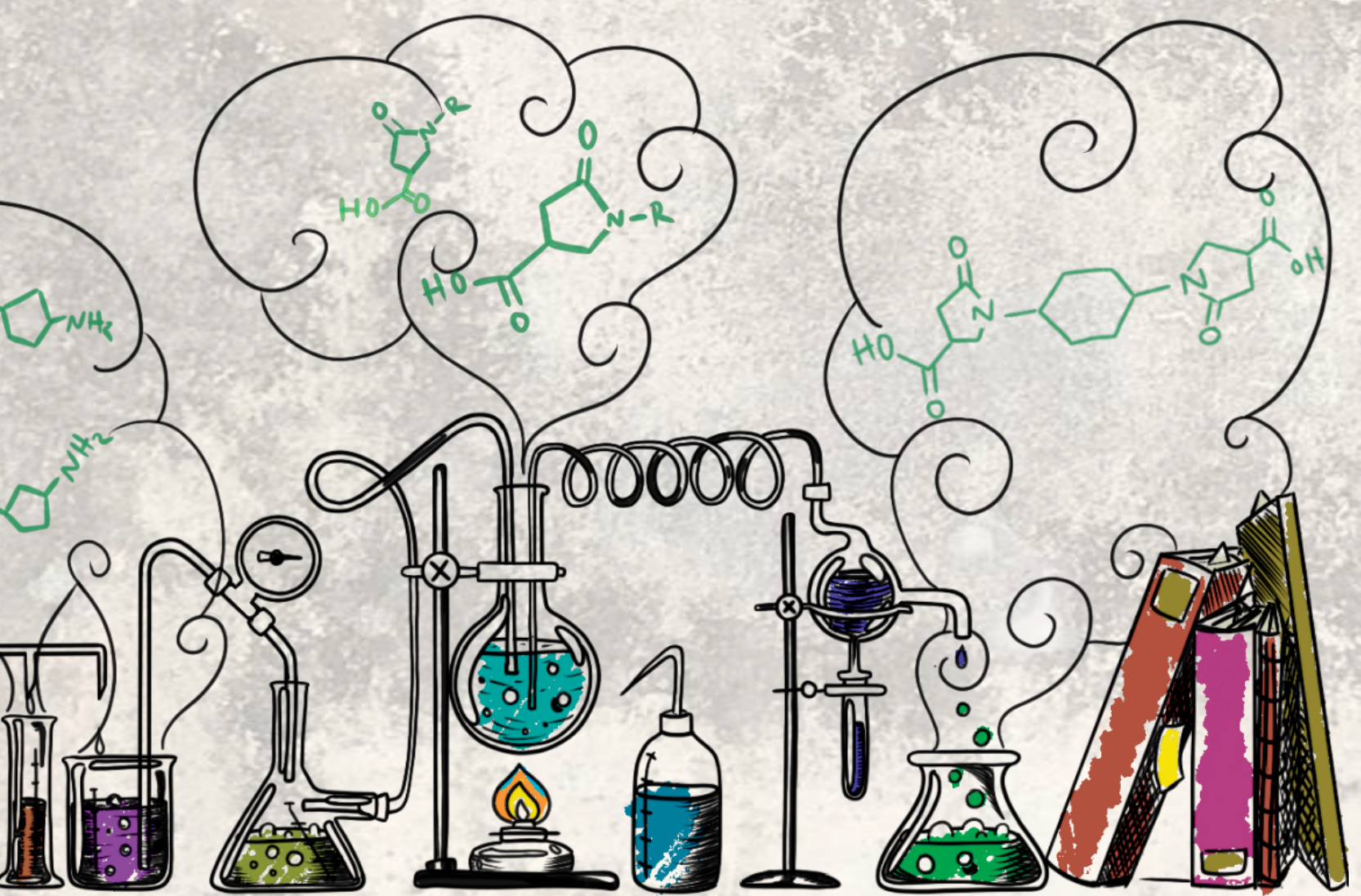




\title{
Renewable cyclic monomers for high- performance biobased polyesters.
}

\author{
PROEFSCHRIFT
}

\author{
ter verkrijging van de graad van doctor \\ aan de Universiteit Maastricht, \\ op gezag van Rector Magnificius, Prof. dr. Rianne M. Letschert, \\ volgens het besluit van het college van Decanen, \\ in het openbaar te verdedigen op \\ vrijdag 01 mei 2020 om 14.00 uur
} door

Geert J. Noordzij 


\section{Promotor}

Prof. dr. S. Rastogi

\section{Co-promotor}

Dr. Ir. C.H.R.M. Wilsens

\section{Beoordelingscommissie}

Prof. dr. Maarten Honing (Voorzitter)

Dr. Matthew Baker

Prof. dr. Rint Sijbesma (Eindhoven University of Technology)

Prof. dr. Stefan Bon (Warwick University) 
Renewable cyclic monomers for highperformance biobased polyesters.

Geert J. Noordzij 
Renewable cyclic monomers for high-performance biobased polyesters.

G.J. Noordzij, Maastricht University, 2020.

ISBN: 9789464021929

Cover art by Cris García Saravia Ortiz de Montellano

Design and layout by Geert Noordzij

This work was performed under the framework of Chemelot InSciTe and is supported by contributions from the European Regional Development Fund (ERDF) within the framework of OP-Zuid and with contributions from the province of Brabant and Limburg and the Dutch Ministry of Economy. 


\section{Table of Contents}

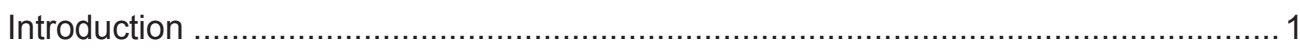

Chapter 1 Small-scale screening of novel biobased monomers.......................... 12

Chapter 2 The curious case of 1,3-cyclopentanediol.................................... 38

Chapter 3 1,3-cyclopentanediol as comonomer in poly(ethylene furanoate) (PEF)... 64

Chapter 4 Cascade aza-Michael addition-cyclizations ................................. 80

Chapter 5 The Aza-Michael reaction: Towards semi-crystalline polymers from renewable itaconic acid and diamines ......................................... 108

Chapter 6 On the mechanical performance of semi-crystalline bis(pyrrolidone) based polymers derived from itaconic acid........................................ 144

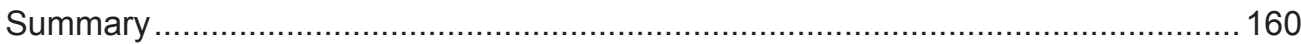

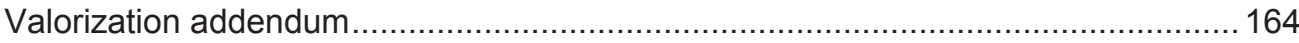

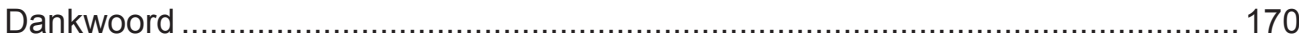

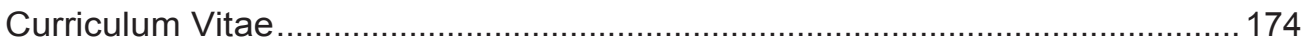

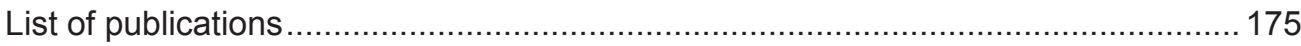





\section{Introduction}

I remember clearly that when I first started doing research in sustainability in chemistry, and more specifically sustainable polymer chemistry, that most publications, PhD defenses and (public) lectures started with arguing why we need to make plastics from renewable resources rather than from nonrenewable fossil resources. The main argument usually was something along the lines of: "due to dwindling supplies of oil", or "it is inevitable that we will run out of oil". Which is de facto a true argument: IF we ever run out of oil (we're probably still 'good' for another 100 years), then yes we cannot make these plastics anymore, and therefore we need to do research in alternative resources. However, this also implies that by the time we run out of oil, we still use the same technology to prepare our polymer materials, which is unlikely. Another argument used to be regarding the "ever increasing" oil price, (then at $90 \$ /$ barrel) makes conventional plastic expensive, and in the future renewable resources will be cheaper. Back then it was estimated that an oil price of approximately $110 \$ /$ barrel was needed for renewable resources to become price competitive. Well the latter was quickly proven "wrong" as the oil price plummeted to $33 \$$ /barrel in 2014 , and is still well below those high prices. ${ }^{1}$ The fact, and the only reason that truly matters, is that we should stop using fossil resources (not limited to the production of plastics), because we need to stop putting more carbon dioxide $\left(\mathrm{CO}_{2}\right)$ in the atmosphere. And the timeline to stop doing so is much much shorter than running out of fossil resources to burn.

The $\mathrm{CO}_{2}$ comes directly from burning fossil fuel resources for our need for energy and indirectly from the production of materials (and plastics). These fossil fuels have been formed over the course of millions of years by decomposing plant matter and microorganisms (not T-Rex), and have been stored underground, away from the current atmospheric cycle. However by using the fossil resources we are adding extra $\mathrm{CO}_{2}$ (anthropogenic carbon emissions) in the atmosphere, on top of existing natural cycle of $\mathrm{CO}_{2}$ currently in place. ${ }^{2}$ The anthropogenic carbon emissions have led to a manmade climate change, which we are currently already experiencing in changing weather patterns ${ }^{3}$; e.g. heat- and cool-fronts in unlikely places, droughts and floods in places and at frequencies never seen before. These changing weather patterns are affecting crop growth, directly affecting the food source affecting people world-wide. ${ }^{4}$ This knowledge is the basis for climate change activists already since the 1970 's ${ }^{5}$, and the International Panel for Climate Change (IPCC) since 1988 to warn and advice on policies regarding fossil fuels. Climate models predict that with the continuation of the current use of resources (business as usual), we are facing a potential climate change and global warming of $3-5{ }^{\circ} \mathrm{C}$ (Figure 1)..$^{6,7}$ The predicted rise in temperature will likely cause harsh changes for the natural ecosystem. To attempt to limit the consequences, during the Paris Agreement in 2016 it was decided we should aim for a maximum of $1.5-2{ }^{\circ} \mathrm{C}$ heating 
to stay at "safe" levels. ${ }^{8}$ However, since this Paris Agreement there is still no slowing down in the amount of $\mathrm{CO}_{2}$ we are pumping in the atmosphere.

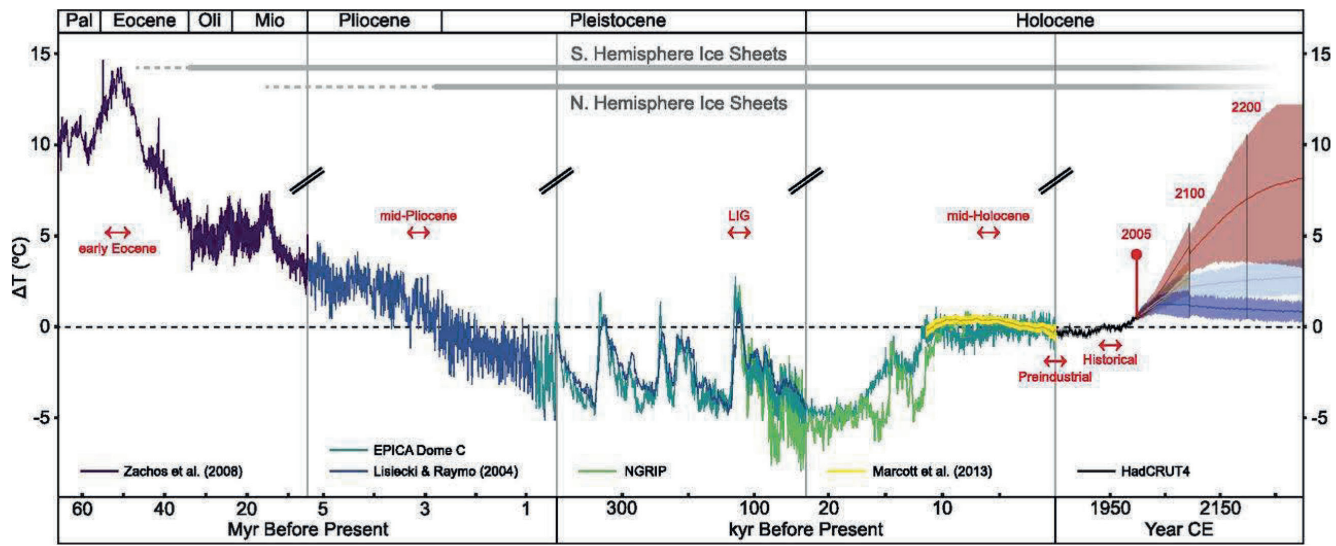

Figure 1. Average worldwide temperature trends over the past 65 million years. Visible is the relatively stable temperature during the mid-Holocene (ca. 12000 BC until present), during which the human civilization was able to settle worldwide. Global warming predictions induced by human activities show a predicted average increase in temperature by $3-5^{\circ} \mathrm{C}$ with by the year 2100 (right, red line) if we continue like this. The temperature goals of the Paris Agreement (right, blue) with an average increase of $1.5-2{ }^{\circ} \mathrm{C}$, and the projected temperature pathway if we revert global warming (right, purple). Either way it is clearly visible how the earth is rapidly warming, and worst case (with business as usual) to potentially average temperatures not seen in 50 million years. ${ }^{6}$ The change in average global temperatures will change weather patterns and strongly influence which type of flora and fauna can thrive, and such human induced climate change directly influences all ecosystems and living species on this planet.

The vast majority of the extracted fossil fuels are directly burned for energy; e.g. coal at power stations, petrol/diesel/kerosene for transportation, and gas for heat and cooking at home. So if anything, in terms of going towards a more sustainable future with limited climate change, an energy transition is highly needed. ${ }^{9}$ Apart from the need for an energy transition to use less fossil fuels, about $15 \%$ of fossil fuel (mostly oil) are used in the petrochemical industry, of which about $8 \%$ end up in the production of plastics. Though this $15 \%$ of petrochemicals may not sound much, it still is totaling to 900 million tons of oil, out of which approximately 400 million tons of plastics are currently made per year. With an expected growth to 1200 million tons of plastic produced per year in 2050. ${ }^{10}$ These petrochemicals and plastics, inevitably, will eventually all break down to $\mathrm{CO}_{2}$. So also here, in terms of sustainability regarding anthropogenic carbon, there is a significant gain to be had by looking at other sources to produce plastic materials.

But if fossil oil can't be used as starting material, then what can be used at such scale which will not disrupt the natural $\mathrm{CO}_{2}$ balance? Here, as carbon source, we should go back to what is annually supplied as steady carbon source, without adding new $\mathrm{CO}_{2}$ to the atmosphere: natural growth of biomass. It is estimated that the annual growth in biomass due to photosynthesis (including crops, grasses, trees, algae) is about 150 $190 \times 10^{9}$ tons. ${ }^{11}$ Further estimates from the World Bioenergy Association is that plants convert solar energy and carbon dioxide from the atmosphere into biomass, at a rate roughly 80 times the global energy demand. ${ }^{12-14}$ Currently roughly $10 \%$ of the annual biomass growth is from agriculture ${ }^{13}$, of which $32 \%$ is for food, $60 \%$ for animal feed, $4 \%$ material use, and $4 \%$ energy use. ${ }^{15}$ Furthermore, from the feed and food production, the 
actual food (corn, wheat, rice, citrus, etc.) is typically around $50 \%$ of the entire plant dry weight (e.g. corn $^{16}$, citrus ${ }^{17}$, wheat $\left.{ }^{18}\right)$. The (left-over) plant consists mostly out of cellulose (40-50\%), hemi-cellulose (15-25\%), lignin (20-25\%), and other components (5-10\%). ${ }^{19}$ Though the ratios can differ; e.g. woody biomass has a higher lignin concentration than herbaceous biomass.

In principle all these plant structures can be used as chemical feedstock to produce biobased monomers and polymers. However, currently mostly sugar and starch based produce are used to produce commercially available biobased plastics such as polylactide (PLA), poly(ethylene furanoate) (PEF) and bio-polyethylene (bio-PE). Whether this direct use from the food feedstock is good or bad is under constant debate $^{20}$, however presently only about $0.02 \%$ of the arable land area is used to produce the current bio-based plastics (1-2\% of all plastics made). ${ }^{21,22}$ If all plastics would be made from biobased resources, the demand for feedstock would be in the order of $5 \%$ of all agricultural biomass produced. ${ }^{23}$ This is still a relatively small portion on the food feedstock. However, this $5 \%$ demand is not likely to happen since there is continues technology development to use secondary biomass sources for chemical building blocks. ${ }^{23,24}$ For example cellulose (secondary biomass) can be used to prepare glucose as a chemical feedstock to produce the same biobased plastics such as PLA, PEF and bio-PE without interfering with the food feedstock. ${ }^{25}$ But also the other sugars present in hemi-cellulose ${ }^{26}$, and potential building blocks from lignin ${ }^{26,27}$ will most likely drive the biobased monomer market, without competition for food. ${ }^{15}$

The chemical structure and complexity differs per plant component. From a chemical point of view first generation biomass (food biomass) is the best source for production of chemicals because the starting material is very pure. For example, sugar from sugar beet or cane yields high quality sucrose-syrup which is industrially easy to process in pure sucrose (a disaccharide). Starch-produce as potatoes, rice and wheat are mostly amylose and amylopectin (a polysaccharide), both polymeric forms of $\alpha$-glucose, which is industrially easy to process in pure glucose (a monosaccharide). The rest of the plant (stems, stalks, roots, etc.) is used as second generation biomass (lignocellulosic biomass), this biomass is chemically more diverse, as is depicted in Figure $2 .{ }^{28}$ The structural components of the plant, the cell wall, consist of microfibrils (Figure 2, middle), which are intertwined structures of cellulose, hemi-cellulose and lignin. Cellulose is a semi-crystalline polysaccharide of $\beta$-glucose (Figure 2 , middle right), hemi-cellulose is polysaccharide consisting out of a mixture of $\mathrm{C}_{5}$ and $\mathrm{C}_{6}$ sugars (Figure 2, bottom). Lastly, lignin is a complex mixture of several aromatic/phenolic building blocks (Figure 2, middle left). Since the structural components are intertwined, it is more difficult to directly isolate these components into chemically pure streams, which typically is desired by the chemical industry. Though a lot of effort is made in valorizing the lignocellulosic biomass, focusing on extracting the individual components ${ }^{11,29-31}$, or complete fermentation in biorefineries. ${ }^{17,32,33}$ 


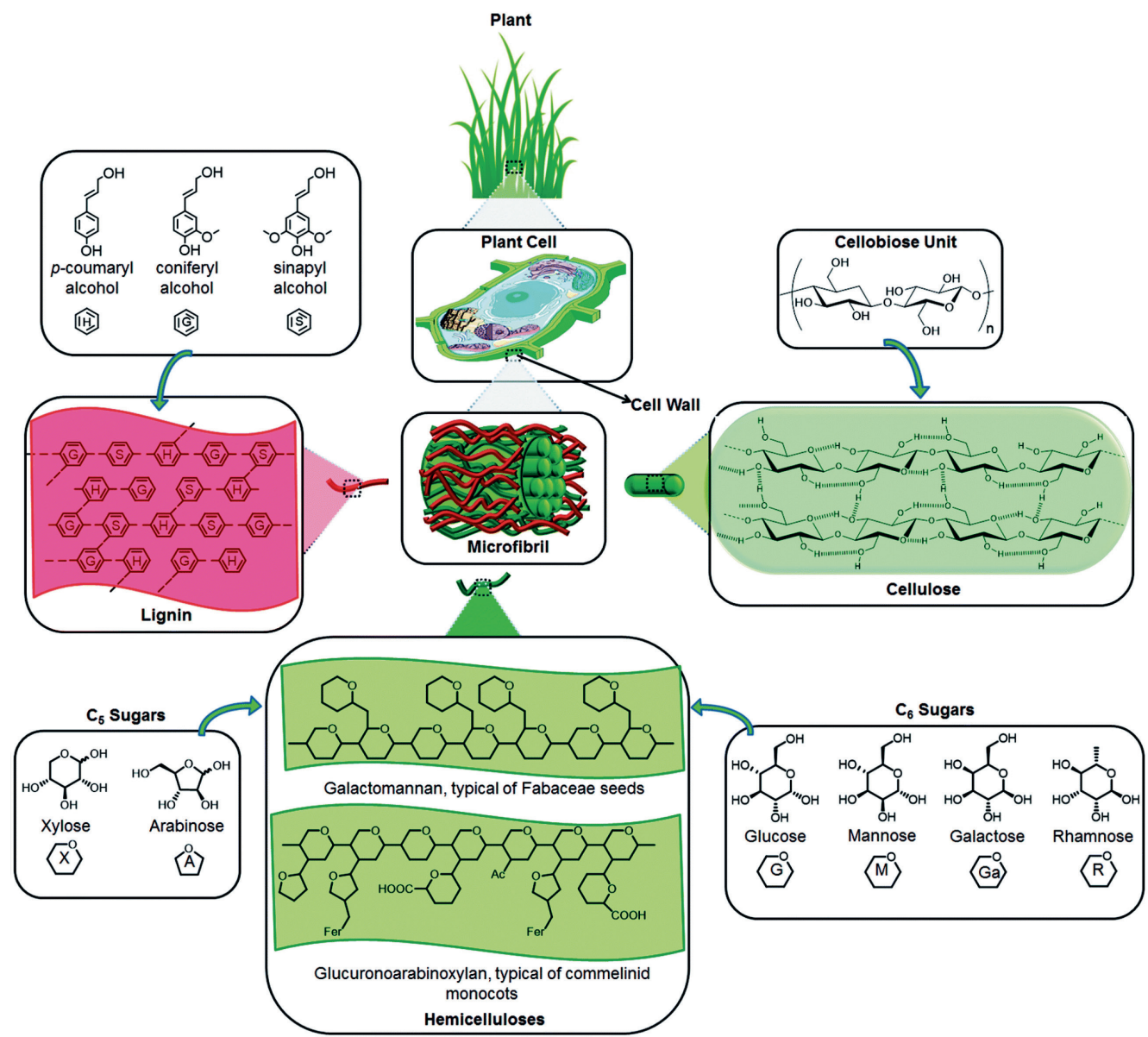

Figure 2. Schematic overview of the structural components of lignocellulosic biomass: Cellulose ( $\beta$-glucose), hemicellulose (mix of $\mathrm{C}_{5}$ and $\mathrm{C}_{6}$ sugars), and lignin (a complex macromolecule of aromatic and phenolics). Image from Isikgor and Becer. ${ }^{28}$

The raw material available from the lignocellulosic feedstock are composed out of molecules with a strong chemical diversity as compared to petrochemical building blocks. The biobased molecules contains many hetero-atoms, reactive and sensitive groups, and stereochemistry ${ }^{34}$, which are often not (directly) compatible with the existing chemical plants. ${ }^{26}$ The challenge remains on how to use the lignocellulosic feedstock to be compatible with the existing infrastructure of the chemical industry and downstream processing. ${ }^{35}$ This is partially circumvented by transforming biomass into the same building blocks as can be obtained from petrochemical resources to produce "drop-in" chemicals. This can for example be found in the development of biobased PE and PET. However, in these processes often a large part of the original molecule is "lost", leading to a low atom efficiency, having a negative impact on the overall sustainability. ${ }^{36}$ With a low atom efficiency relatively more biomass feedstock is required, which can lead a higher strain on crop growth leading to a relatively high load on e.g. water needed and ecotoxicity scores, as is the case for biobased $\mathrm{PE}^{37}$ and $\mathrm{PET}^{38}$. So even though important 
commodity plastics can be made from renewable biomass, they are not by definition more sustainable, and a careful balance needs to be made to decide on the right path to take in the long run. ${ }^{39}$

Alternatively, a large part of chemical research is focused on using the chemically diverse starting materials towards novel functional materials, without changing the original structure too much. Such new building blocks can give rise to completely new classes of bio-based polymers, as is seen in the development of biobased polymers such as PEF and PLA. Chemical transformation of the lignocellulosic feedstock is mostly done via biotechnology to produce new building blocks such as lactic acid and itaconic acid from glucose and other sugars. However, catalytic chemical transformations are also used to produce novel monomers such as 2,5-FDCA from glucose and fructose. Lignin based feedstock are often depolymerized in functional monomers via microbial conversion, oxidation, or pyrolysis, each leading to a different subset of monomers. ${ }^{11}$ There is a vast amount published research on newly developed monomers from lignocellulosic biomass, which have been extensively reviewed over the past years. ${ }^{28,39-43}$ With the most complete dataset running up to 435 different chemicals and materials ${ }^{44}$, it is well out of the scope of this thesis to attempt to summarize them. Though to give a flavor, a schematic overview produced by the nova-Institute is given which displays several key intermediates on going from biomass to polymers (Figure 3). ${ }^{1}$ What is interesting to note is that glucose is at the center stage for most of the intermediates leading to a wide variety of polymeric end-products. Both starch and sacharose (sucrose), as well as lignocellulose lead to glucose, so both primary and secondary biomass sources can be used to make the same polymeric end-products via glucose.

After extraction of high purity streams from the lignocellulosic biomass, any remaining or "too complex" streams can still be utilized. Such complex waste streams can further be processed into value added streams via fractionation processes, where the large complex structures are "cracked" to smaller fractions or molecules. ${ }^{27,45,46}$ Examples of such processes are liquefraction of lignin to produce lignin crude oil ${ }^{47}$, from which either useful building blocks can be extracted, or be used as fuel. ${ }^{46}$ Woody biomass can be fractionated via torrefraction into BTX building blocks. ${ }^{48}$ Further fractionation (higher temperatures) via gassefraction can break down the biomass to small molecules which can result in the production of e.g. methane and syngas, the latter of which can be used again in the production of bulk chemicals ethene and propene, but also fuels. ${ }^{33}$ An interesting side note is that the side-streams of these fractionating processes can be used to process heat (e.g. burning of methane). These processes ensure that as much value is obtained from "left-over" biomass. 


\section{FROM BIOMASS TO POLYMERS}

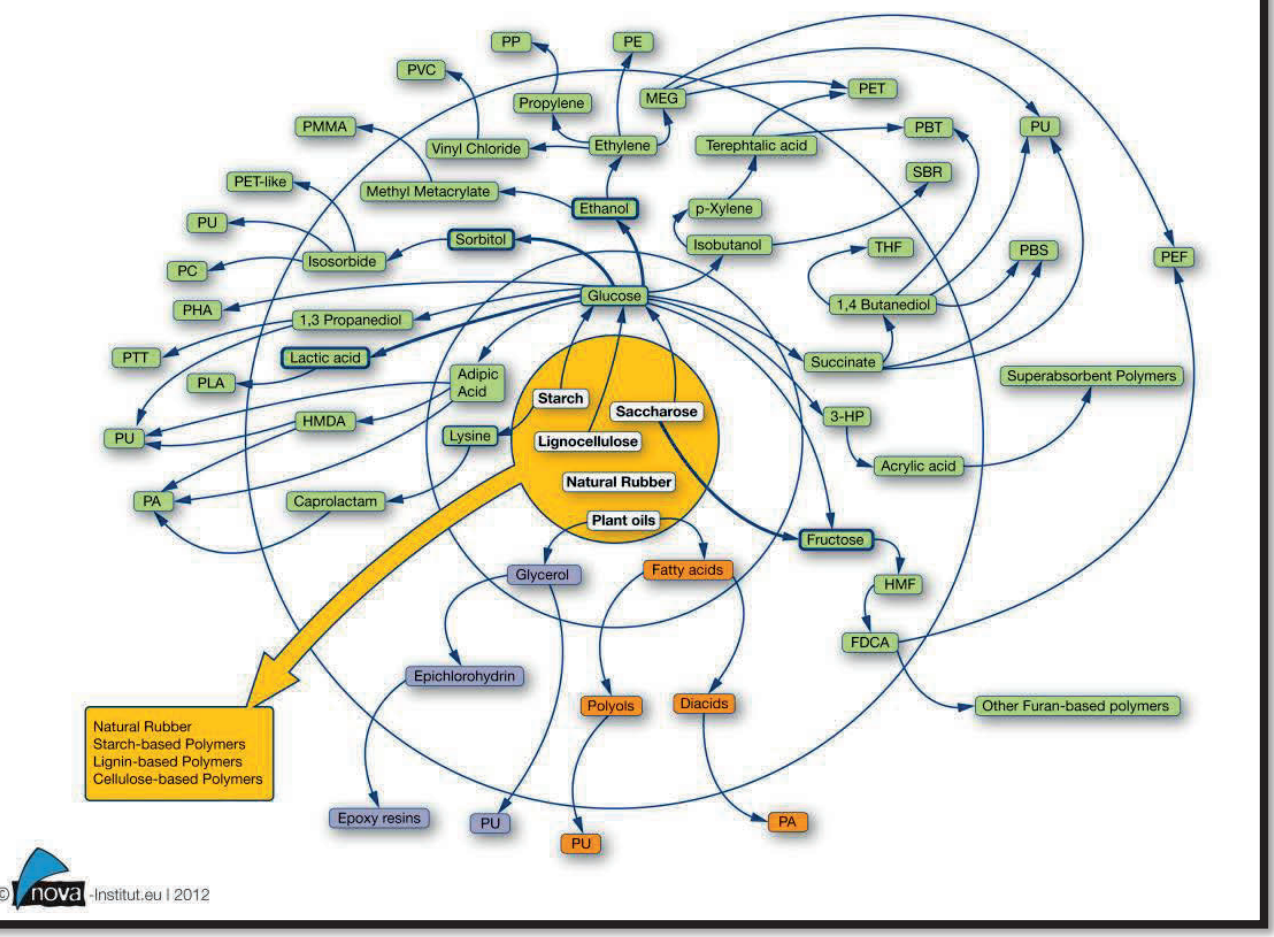

Figure 3. Schematic overview getting going from biomass to intermediates, to chemical building blocks, to polymeric materials. It is important to note that glucose as at the center stage to get to most building blocks for biobased polymeric materials. Glucose can be obtained from both first generation (starch, sugar) and second generation (lignocellulosic) biomass. Image from nova-Institute. ${ }^{1}$

Overall, there are many different routes possible to go from plant biomass to (new) biobased building blocks or renewable energy to (partially) distance ourselves from the fossil fuel dependency. However, for each application a careful consideration needs to be done via a life cycle assessment to assess which pathway is indeed the most sustainable. Moreover, it may be the case that for some applications fossil oil-based plastics will still be the most sustainable choice for some time to come. Nevertheless, with all the ongoing and upcoming research activities and upscaling processes, there will also be growth to be able to supply the growing demand for biobased plastics, both in volume, price, and sustainability. ${ }^{49}$ So indeed the future looks bright and green. 


\section{Introduction InSciTe HORIZONTAL.}

My PhD research was performed under the framework of Chemelot InSciTe, within the HORIZONTAL project. Chemelot InSciTe is a public-private research institute founded by a collaboration between Maastricht University, Eindhoven University of Technology, DSM, and the province of Limburg. The research institute focusses on two major challenges: How to sustain the health of a growing population (biomedical), and how to sustainably produce chemicals, materials, and energy for this growing population (biobased). ${ }^{50}$ Within the biobased program the HORIZONTAL project had simultaneously started with $5 \mathrm{PhD}$ researchers to develop a new route to go from (hemi)cellulosic biomass to specialty monomers for materials (Figure 4). The research starts with catalytic conversion of (hemi)cellulosic biomass (glucose and fructose) and solvent engineering to produce and isolate the intermediates 5-hydroxymethylfurfural (HMF) and furfural (FF). ${ }^{51-53}$ This, and subsequent reactions in the spinning disk reactor was performed by colleagues at the Technical University of Eindhoven. The catalytic conversion of furfural to the intermediate 4-hydroxy-2-cyclopenten-1-one, and the subsequent conversion to either cyclopentanone, or 1,3-cyclopentanediol and 1,3-cyclopentanediamine is performed by a colleague at Maastricht University. The subsequent use and practical applications of 1,3-cyclopentanediol and 1,3-cyclopentanediamine in novel biobased polymers is described in this thesis.

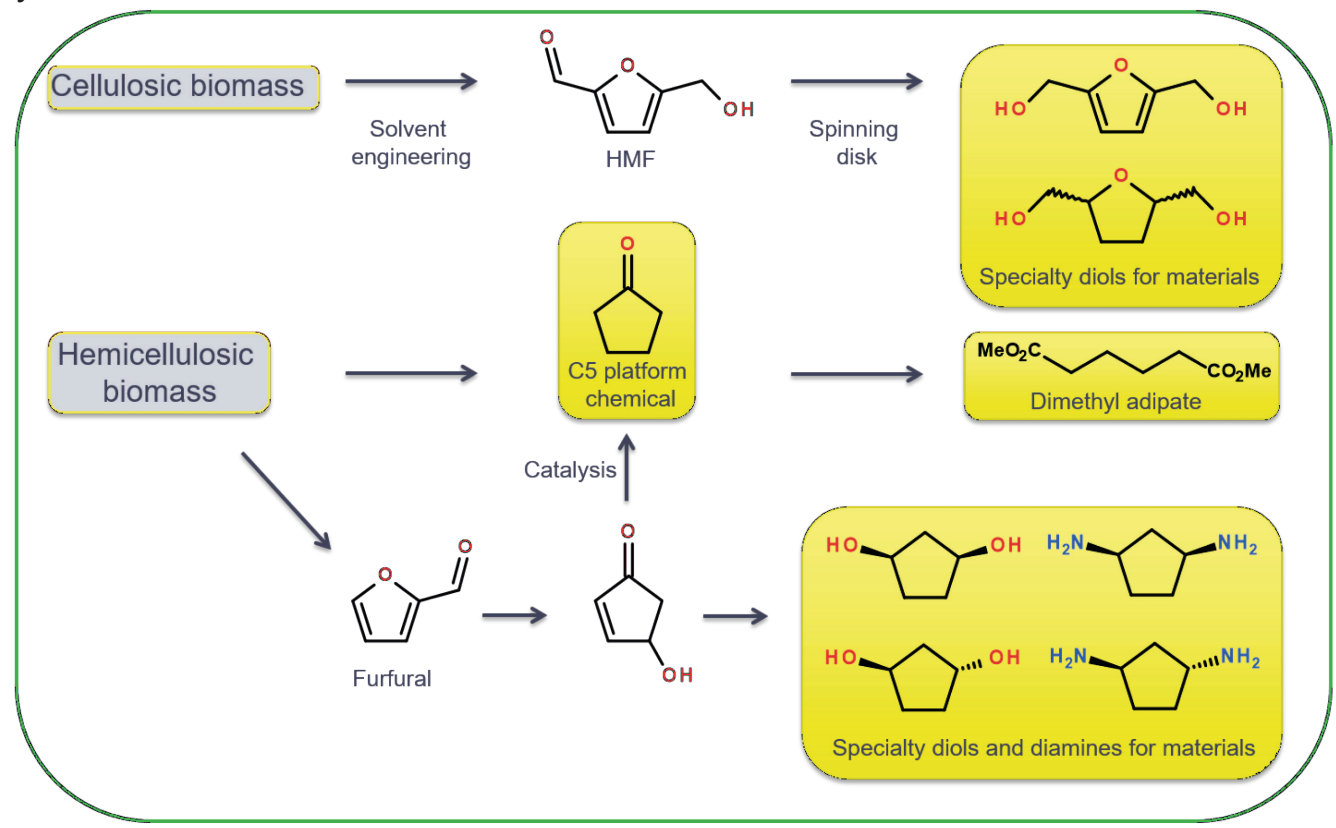

Figure 4. Schematic overview of the HORIZONTAL project of Chemelot InSciTe: Developing a novel production and isolation route from (hemi)cellulosic biomass (glucose, fructose) to key-intermediates. Followed by catalytic conversion to specialty diols and diamines and their subsequent use in novel biobased polymers, of which the latter is described in this thesis. 
As is shown previously in the introduction, the potential intermediates and building blocks which can be produced from ligno-cellulosic, and especially (hemi)cellulosic biomass is enormous. Sometimes the intermediates or products are clear targets, such as drop-in chemicals for the existing petrochemical industry. Often the targeted intermediates or building blocks are products of design based on known and/or hypothetical (bio)chemical transformations, and can lead to a plethora of new materials. However, it is often unknown if such building blocks are also useful, especially in the field of polymer materials. Not only whether new building blocks are easy or hard to polymerize, but also -when polymerized- what the associated polymer properties are. With smart product design of the envisioned building blocks to obtain desired properties several factors are taken into account: chemical functionality, stereochemistry, rigidity and stability. Often, the currently well-developed building blocks or polymers are taken as reference to assess what should be targeted. This can for example be seen in the production of biobased 2,5furandicarboxylic acid, which is regarded as the biobased alternative for terephthalic acid. And the polymer thereof, poly(ethylene furanoate) (PEF) is regarded as the biobased alternative for poly(ethylene terephthalate) $(P E T)^{54}$, which is currently under development by Avantium (NL).

The common goal for the polymers envisioned in the HORIZONTAL project, and synthesized and described in this work, is to prepare novel biobased high-performance polymers. 1,3-Cyclopentanediol and 1,3-cyclopentanediamine are chosen because of their structural similarity to industrially important cyclic monomers such as 1,4-cyclohexanediol, 1,4-cyclohexanedimethanol, and 1,4-cyclohexanediamine. The polymerization and comparison of these monomers leads to a good understanding of the structure-property relationship between monomer and polymer properties, wich is a common theme throughout this thesis. The low availability of starting material is common in the development of new biobased building blocks, and this issue is being addressed in chapter 1 in this thesis with the development of a small-scale high-throughput polycondensation reactor. This way the structure-property relationship of 1,3-cyclopentanediol could be fully characterized (chapters $1 \& 2$ ) with just a few grams of starting material. The most promising (co-)polyesters out of this small-scale study were polymerized in larger scale, from which polymeric films were made and subsequently tested for gas-barrier properties (chapter 3 ). The second part of this thesis (chapters 46) is focused on 1,3-cyclopentanediamine, which proved to be too (thermally) unstable to use in direct polycondensation reactions. Therefore, the diamines are transformed into new intermediates via a cascade aza-Michael - cyclization (chapter 4) with the renewable monomer itaconic acid. The resulting di-acid intermediate is polymerized with a variety of diols into new polyesters to perform a new structure-property relationship study (chapter 5). From which the most promising polyesters were synthesized on larger scale to study the effect of processing and crystallinity on the mechanical performance (chapter 6). 


\section{References}

1. Dammer, L., Carus, M., Raschka, A. \& Scholz, L. Market Developments of and Opportunities for biobased products and chemicals. Markets and Trends of Bio-based Products (2013).

2. Ciais, P. et al. Carbon and other biogeochemical cycles. Clim. Chang. 2013 Phys. Sci. Basis Work. Gr. I Contrib. to Fifth Assess. Rep. Intergov. Panel Clim. Chang. 9781107057, 465-570 (2013).

3. R.K. Pachauri, L. A. M. and C. W. T. Climate Change 2013 - The Physical Science Basis. Ipcc (Cambridge University Press, 2014). doi:10.1017/CBO9781107415324

4. Melillo, J. M., Richmond, T. C. \& Yohe, G. W. Climate Change Impacts in the United States: The Third National Climate Assessment. (2014). doi:10.7930/J0Z31WJ2

5. Meadows, D. H., Meadows, D. L., Randers, J. \& Behrens, W. W. The limits to growth. (Universe Books, 1972).

6. Burke, K. D. et al. Pliocene and Eocene provide best analogs for near-future climates. Proc. Natl. Acad. Sci. 115, 1328813293 (2018).

7. $\mathrm{CO} 2$ and other greenhouse gas emissions. Available at: https://ourworldindata.org/co2-and-other-greenhouse-gasemissions. (Accessed: 30th October 2019)

8. EC. Paris Agreement. Available at: https://ec.europa.eu/clima/policies/international/negotiations/paris_en. (Accessed: 30th October 2019)

9. Kern, F. \& Smith, A. Restructuring energy systems for sustainability? Energy transition policy in the Netherlands. Energy Policy 36, 4093-4103 (2008).

10. WEF. The new plastics economy. Rethinking the future of plastics. (2016).

11. Hodásová, L., Jablonsky, M., Andrea, S. \& Haz, A. Lignin, potential products and their market value. Wood Res. 60, 973-986 (2015).

12. WBA. GLOBAL Biomass Potential Towards 2035. (2016).

13. WBA. WBA Global Bioenergy Statistics 2018. (2018).

14. Tursi, A. A review on biomass: importance, chemistry, classification, and conversion. Biofuel Res. J. 6, 962-979 (2019).

15. Carus, M. \& Dammer, L. Food or non-food: Which agricultural feedstocks are best for industrial uses? (2013). doi:10.1089/ind.2013.1580

16. Roth, G. Corn stover for biofuel production. (2019). Available at: https://farm-energy.extension.org/corn-stover-forbiofuel-production/. (Accessed: 28th October 2019)

17. Pfaltzgraff, L. A., De bruyn, M., Cooper, E. C., Budarin, V. \& Clark, J. H. Food waste biomass: a resource for high-value chemicals. Green Chem. 15, 307 (2013).

18. Sun, R.-C. Cereal Straw as a Resource for Sustainable Biomaterials and Biofuels ... - RunCang Sun - Google Books. (Elsevier, 2010).

19. Zeng, Y., Himmel, M. E. \& Ding, S.-Y. Visualizing chemical functionality in plant cell walls. Biotechnol. Biofuels 10, 263 (2017).

20. Philp, J. C. Biobased Chemicals and Bioplastics: Finding the Right Policy Balance. Ind. Biotechnol. 10, 379-383 (2014).

21. EB. Bioplastics - facts and figures. (2019).

22. EB. Biobased plastics - Fostering a resource efficient circular economy. (2019).

23. van den Oever, M., Molenveld, K., van der Zee, M. \& Bos, H. Bio-based and biodegradable plastics : facts and figures : focus on food packaging in the Netherlands. Residue Reviews (2017). doi:10.18174/408350

24. Bos, H. L. \& Sanders, J. P. M. Raw material demand and sourcing options for the development of a bio-based chemical industry in Europe. Biofuels, Bioprod. Biorefining 7, 246-259 (2013).

25. Deloitte. Opportunities for the fermentation-based chemical industry. (2014).

26. Kühlborn, J., Groß, J. \& Opatz, T. Making natural products from renewable feedstocks: back to the roots? Nat. Prod. Rep. (2019). doi:10.1039/C9NP00040B

27. Schutyser, W. et al. Chemicals from lignin: an interplay of lignocellulose fractionation, depolymerisation, and upgrading. Chem. Soc. Rev. 47, 852-908 (2018).

28. Isikgor, F. H. \& Becer, C. R. Lignocellulosic biomass: a sustainable platform for the production of bio-based chemicals and polymers. Polym. Chem. 6, 4497-4559 (2015).

29. Sun, Z., Fridrich, B., de Santi, A., Elangovan, S. \& Barta, K. Bright Side of Lignin Depolymerization: Toward New Platform Chemicals. Chem. Rev. 118, 614-678 (2018).

30. Chaturvedi, V. \& Verma, P. An overview of key pretreatment processes employed for bioconversion of lignocellulosic biomass into biofuels and value added products. 3 Biotech 3, 415-431 (2013).

31. Sheldon, R. A. The Road to Biorenewables: Carbohydrates to Commodity Chemicals. ACS Sustain. Chem. Eng. 6, 4464-4480 (2018).

32. Coma, M. et al. Organic waste as a sustainable feedstock for platform chemicals. Faraday Discuss. 202, 175-195 (2017).

33. Erickson, B., Nelson \& Winters, P. Perspective on opportunities in industrial biotechnology in renewable chemicals. Biotechnol. J. 7, 176-185 (2012).

34. Hülsey, M. J., Yang, H. \& Yan, N. Sustainable Routes for the Synthesis of Renewable Heteroatom-Containing Chemicals. ACS Sustain. Chem. Eng. 6, 5694-5707 (2018).

35. Jang, Y.-S. et al. Bio-based production of C2-C6 platform chemicals. Biotechnol. Bioeng. 109, 2437-2459 (2012).

36. Sara, M., Brar, S. K. \& Blais, J. F. Production of Drop-In and Novel Bio-Based Platform Chemicals. in Platform Chemical Biorefinery 249-283 (Elsevier, 2016). doi:10.1016/B978-0-12-802980-0.00014-6

37. Belboom, S. \& Léonard, A. Does biobased polymer achieve better environmental impacts than fossil polymer? Comparison of fossil HDPE and biobased HDPE produced from sugar beet and wheat. Biomass and Bioenergy $\mathbf{8 5}$, 159-167 (2016). 
38. Chen, L., Pelton, R. E. O. \& Smith, T. M. Comparative life cycle assessment of fossil and bio-based polyethylene terephthalate (PET) bottles. J. Clean. Prod. 137, 667-676 (2016).

39. Llevot, A. et al. Renewability is not Enough: Recent Advances in the Sustainable Synthesis of Biomass-Derived Monomers and Polymers. Chem. - A Eur. J. 22, 11510-11521 (2016).

40. Nakajima, H., Dijkstra, P. \& Loos, K. The Recent Developments in Biobased Polymers toward General and Engineering Applications: Polymers that are Upgraded from Biodegradable Polymers, Analogous to Petroleum-Derived Polymers, and Newly Developed. Polymers (Basel). 9, 523 (2017).

41. Delidovich, I. et al. Alternative Monomers Based on Lignocellulose and Their Use for Polymer Production. Chem. Rev. acs.chemrev.5b00354 (2015). doi:10.1021/acs.chemrev.5b00354

42. Gandini, A. \& Lacerda, T. M. From monomers to polymers from renewable resources: Recent advances. Prog. Polym. Sci. 48, 1-39 (2015).

43. Corma Canos, A., Iborra, S. \& Velty, A. Chemical routes for the transformation of biomass into chemicals. Chem. Rev. 107, 2411-2502 (2007).

44. Lee, S. Y. et al. A comprehensive metabolic map for production of bio-based chemicals. Nat. Catal. 2, 18-33 (2019).

45. de Wild, P. J. Biomass pyrolysis for chemicals. (Rijksuniversiteit Groningen, 2011).

46. Mohan, D., Pittman, C. U. \& Steele, P. H. Pyrolysis of Wood/Biomass for Bio-oil: A Critical Review. Energy \& Fuels 20, 848-889 (2006).

47. Vertoto - Lignin Crude Oil. Available at: wwww.vertoro.com. (Accessed: 30th October 2019)

48. BioBTX. Available at: www.biobtx.com. (Accessed: 30th October 2019)

49. Bomtempo, J.-V., Chaves Alves, F. \& de Almeida Oroski, F. Developing new platform chemicals: what is required for a new bio-based molecule to become a platform chemical in the bioeconomy? Faraday Discuss. 202, 213-225 (2017).

50. Chemelot InSciTe. Available at: https://www.chemelot-inscite.com/en/about-us/why-we-do-it. (Accessed: 29th October 2019)

51. Papaioannou, M., Kleijwegt, R. J. T., van der Schaaf, J. \& Neira d'Angelo, M. F. Furfural Production by Continuous Reactive Extraction in a Millireactor under the Taylor Flow Regime. Ind. Eng. Chem. Res. 58, 16106-16115 (2019).

52. Dietz, C. H. J. T., Kroon, M. C., van Sint Annaland, M. \& Gallucci, F. Thermophysical Properties and Solubility of Different Sugar-Derived Molecules in Deep Eutectic Solvents. J. Chem. Eng. Data 62, 3633-3641 (2017).

53. Krzelj, V., van Kampen, J., van der Schaaf, J. \& Neira d'Angelo, M. F. Furfural Production by Reactive Stripping: Process Optimization by a Combined Modeling and Experimental Approach. Ind. Eng. Chem. Res. 58, 16126-16137 (2019).

54. Eerhart, A. J. J. E., Faaij, A. P. C. \& Patel, M. K. Replacing fossil based PET with biobased PEF; process analysis, energy and GHG balance. Energy Environ. Sci. 5, 6407 (2012). 


\section{Chapter 1 Small-scale screening of novel biobased monomers}

This chapter is based on the publication:

Small-scale screening of novel biobased monomers: the curious case of 1,3-cyclopentanediol.

G.J. Noordzija,b, C.H.J.T. Dietz ${ }^{c, b}$, N. Leonéa ${ }^{a}$ C.H.R.M. Wilsens ${ }^{a}$, S. Rastogia

RSC Advances, 2018, 8, 39818

DOI: $10.1039 / c 8 r a 08811 \mathrm{j}$

a) Aachen-Maastricht Institute of Biobased Materials (AMIBM), Faculty of Science and Engineering, Maastricht University, Brightlands Chemelot Campus, 6167 RD Geleen, The Netherlands.

b) Chemelot InSciTe, Urmonderbaan 20F, NL-6167 RD Geleen, The Netherlands.

c) Inorganic Membranes and Membrane Reactors, Dept. Chemical Engineering and Chemistry, Eindhoven University of Technology, PO Box 513, Eindhoven, The Netherlands.

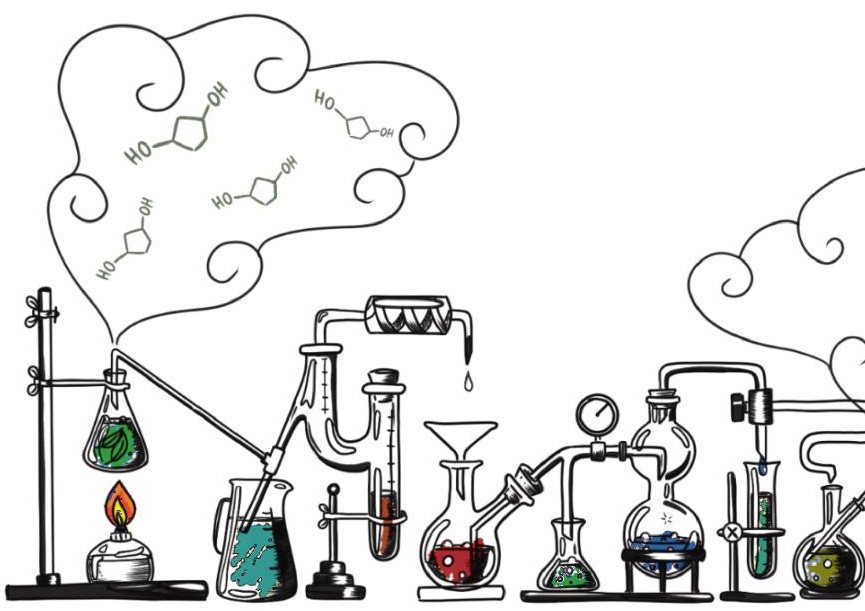




\section{Abstract}

In this work, we report on a novel method for small-scale (<10 mg) polycondensation reactions in a 57-parallel batch-reactor, and consecutive analysis of novel polyesters based on the potentially renewable 1,3-cyclopentanediol (CPdiol). Trimer pre-polyesters have been synthesized from the corresponding acid-chlorides with diol monomers, which in turn were successfully used in thin-film polycondensation reactions, without suffering from monomer evaporation and sublimation otherwise experienced in thin-film polycondensation reactions. TGA analysis was used to screen for the optimal polymerization temperature and catalyst for polymerization in the batch-reactor. The described method was used to synthesize a range of novel (co-)polyesters based on CPdiol at $180-210{ }^{\circ} \mathrm{C}$, yielding molecular weights well above 10 $\mathrm{kg} / \mathrm{mol}$. Furthermore, CPdiol as potential new biobased building block was compared to the commercially relevant cycloaliphatic diols 1,4-cyclohexanediol (CHdiol) and 1,4cyclohexanedimethanol (CHdmol). Polyesters with CPdiol exhibited a lower $T_{\mathrm{g}}$ and $T_{\mathrm{m}}$ as compared to the CHdiol counterpart, but strikingly similar to polyesters with the $\mathrm{CHdmol}$ counterpart, albeit with a somewhat hampered crystallization with the short chain $\left(\mathrm{C}_{6}\right)$ aliphatic spacer. Lastly, MALDI-ToF-MS analysis showed that random copolyesters could readily be synthesized by controlling the stoichiometry of trimer pre-polyester mix at the start of the thinfilm polycondensation.

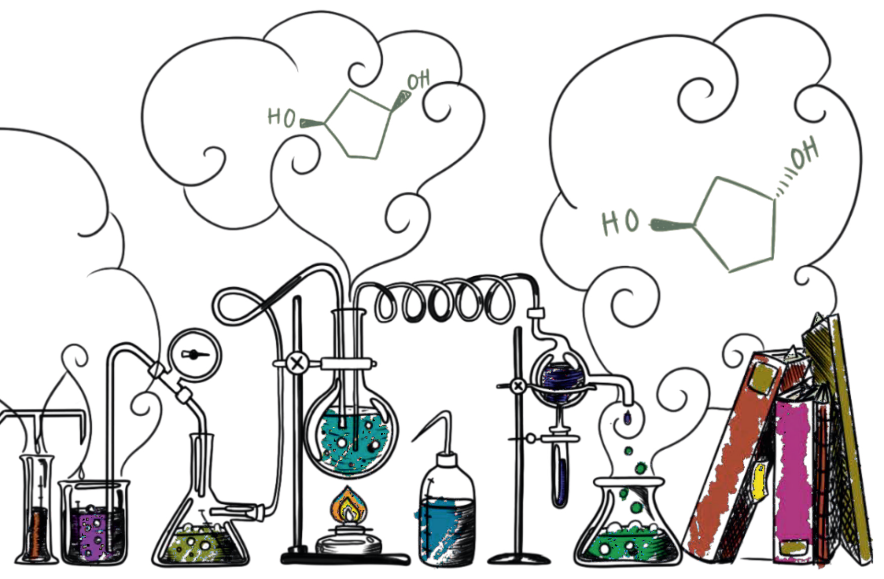




\section{Introduction}

Worldwide strenuous efforts are taking place to replace oil-based monomers with renewable biomass-based monomers, for both "drop-in" purposes and as new monomers. ${ }^{1-5}$ However, in particular for new building blocks, it is not assured that these monomers -or the resulting polymeric materials- will function as desired. In order to fully assess such monomers and polymers, generally large amounts (e.g. several kilos) are required to conduct repeatable processing and characterization tests. Unfortunately, academic research often leads to new monomers obtained via e.g. new (bio)catalytic processes having yields in gram scale, or lower. Another major limitation for translations of lab scale synthesis to large scale are the required revenues: significant investment in both time and money is needed to develop new monomers and polymers on a larger scale. To overcome this mismatch in scale, a viable assessment method is desired to polymerize and qualify monomers on a small scale.

High-throughput screening equipment is a well-known tool in the fast screening of molecules in e.g. organic synthesis, drug-discovery, and cell-experiments. Also in the field of polymer synthesis, semi-automated high-throughput techniques have been used in solution polymerization, ${ }^{6,7}$ olefin polymerization, ${ }^{8}$ and melt-polycondensation. ${ }^{9,10}$ For example, a small-scale high-throughput melt-polycondensation experiment has been described by Gruter et a/ ${ }^{11}$ for the evaluation of catalyst effectivity in the synthesis of poly(ethylene 2,5-furandicarboxylate) (PEF), commonly considered to be the renewable replacement of poly(ethylene terephthalate) (PET). In general, such screening techniques can lead to faster catalyst development, but can also provide systematic identification of structure-property relation in polymers by varying monomer compositions. An added benefit of small-scale screening experiments is a minimal requirement of monomer availability, making this an attractive technique for assessing the potential of new building blocks. Despite the advantages of high-throughput experimentation, full automation for polymerization reactions can still be challenging due to the time restrictions for polymer characterization. For example, important polymer characterization techniques such as gel-permeating chromatography (GPC) or differential scanning calorimetry (DSC) require relatively long analysis times, potentially becoming the bottleneck in high-throughput screening processes.

The group of Zhang et al. ${ }^{12}$ has developed a new industrially scalable and cost-effective synthesis route to 1,3-cyclopentanediol (CPdiol) from biomass. These authors reported that CPdiol, usually obtained from non-renewable cyclopentadiene, could be obtained from the aqueous phase rearrangement of furfuryl alcohol, followed by hydrogenation over RANEY® Ni Scheme 1-1). CPdiol was obtained as a mixture of cis and trans isomers, which could be further purified via fractional distillation under vacuum. 


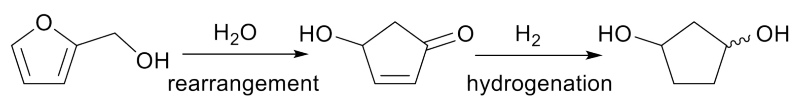

Scheme 1-1. Reaction pathway for the synthesis of 1,3-cyclopentanediol from renewable furfuryl alcohol. ${ }^{12}$

Cycloaliphatic diols such as 1,4-cyclohexanediol (CHdiol), 1,4-cyclohexanedimethanol (CHdmol), and 1,1,3,3-tetramethyl-2,4-cyclobutanediol (CBdiol) are regularly used as (co-)monomer in the (co-)polymerization of polyesters, often with beneficial effects on $T_{\mathrm{g}}$ and $T_{\mathrm{m}}{ }^{13-17}$ Compared to these six-membered rings, the five-membered cyclo-aliphatic ring in CPdiol is expected to suppress rigidity and symmetry. ${ }^{18}$ This is due to the steric configuration of the cyclopentane ring, which cannot adopt the well-known 'boat'conformation found in cyclohexane. ${ }^{19}$ Zhang et al. ${ }^{12}$ polymerized 1,3-CPdiol into polyurethanes, and several (enzymatic) transesterification reactions involving various 1,2- and 1,3-CPdiol structures are known. ${ }^{20-22}$ However, to the best of our knowledge, no polycondensation reactions with 1,3-CPdiol have been reported, which might be attributed to the fact that $1,3-\mathrm{CPdiol}$ is currently only available in limited quantities. Therefore, in this study a novel method to study small-scale polycondensation reactions is reported, based on 1,3-CPdiol, which polymer properties are compared to commercially relevant polyesters with 1,4-CHdiol and 1,4-CHdmol groups.

\section{Experimental section}

\section{Materials}

1,3-Cyclopentanediol (15/85 cis/trans), 1,4-cyclohexanedimethanol (38/62 cis/trans), adipoyl chloride, sebacoyl chloride, terephthaloyl chloride, tin(II) 2-ethyl-hexanoate, and anhydrous chloroform, DMF, THF, and toluene were obtained from Sigma Aldrich. Magnesium (IV) sulfate, 4-dimethylaminopyridine and pyridine were obtained from ACROS. 2,5-furan-dicarboxylic acid (FDCA) was obtained from ABCR. 1,4-Cyclohexanediol (46/54 cis/trans) was obtained from Alfa Aesar. Ethylene glycol was obtained from TCl. Standard laboratory solvents were obtained from Biosolve. Deuterated solvents were obtained from Buchem BV (Netherlands). The purchased compounds were used directly without further purification, unless otherwise specified.

\section{Characterization methods}

${ }^{1} \mathrm{H}-\mathrm{NMR}$ and ${ }^{13} \mathrm{C}-\mathrm{NMR}$ spectra were recorded with a Bruker Ultrashield 300 spectrometer $(300 \mathrm{MHz}$ magnetic field). NMR-samples were prepared by dissolving ca. $10 \mathrm{mg}$ of sample in $0.5 \mathrm{~mL}$ deuterated solvent, including dimethyl sulfoxide (DMSO- $\left.d_{6}\right)$, deuterated chloroform $\left(\mathrm{CDCl}_{3}\right)$, and deuterated trifluoroacetic acid ( $d$-TFA). All spectra were referenced against tetramethylsilane (TMS), or residual solvent peak from the deuterated solvent. Molecular weight $\left(M_{\mathrm{n}}, M_{\mathrm{w}}\right)$ and dispersity $(\Xi)$ of the polymers were calculated after gel permeation chromatography (GPC) on a PSS SECcurity GPC system using Agilent 1260 Infinity instrument technology. The GPC system was equipped with two PFG combination medium micro-columns with $7 \mu \mathrm{m}$ particle size $(4.6 \times 250 \mathrm{~mm}$, separation range 100-1.000.000 Da), a PFG combination medium pre-column with $7 \mu \mathrm{m}$ particle size $(4.6 \times 30 \mathrm{~mm})$, and a Refractive Index detector (RI). Distilled 1,1,1,3,3,3-hexafluoroisopropanol (HFIP) containing $0.019 \%$ sodium trifluoroacetate was used as mobile phase at $40{ }^{\circ} \mathrm{C}$, with a $0.3 \mathrm{~mL} / \mathrm{min}$ flow rate. The GPC apparatus was calibrated with poly(methyl methacrylate) standards obtained from PSS. GPC samples were prepared by dissolving $5 \mathrm{mg}$ 
of polymer in $1.5 \mathrm{~mL} \mathrm{HFIP}$ overnight under constant shaking, the samples were filtered over a $0.2 \mu \mathrm{m}$ PTFE syringe filter prior to injection. Thermal stability of compounds and screening of polymerization conditions were performed via thermogravimetric analysis (TGA) using a TA Instruments Q500. Experiments were performed under a nitrogen atmosphere with a heating rate of $10{ }^{\circ} \mathrm{C} / \mathrm{min}$, from room temperature (RT) up to $700{ }^{\circ} \mathrm{C}$. Thermal transition temperatures of the polymers were analyzed via differential scanning calorimetry (DSC) using a TA Instruments DSC Q2000. Typically, two heating and cooling runs were performed at $10^{\circ} \mathrm{C} / \mathrm{min}$, where the first heating was used to erase any thermal history in the samples. The glass-transition $\left(T_{\mathrm{g}}\right)$, melt-transition $\left(T_{\mathrm{m}}\right)$, crystallization $\left(T_{\mathrm{c}}\right)$, and cold-crystallization $\left(T_{\mathrm{cc}}\right)$ temperatures were obtained from the second heating and cooling run. DSC samples were prepared by loading 3-5 mg oven-dried samples in Tzero Hermetic Aluminium pans. Polarized optical microscopy (POM) images were recorded on an Olympus BX53DP 26, equipped with a Linkam HFSX350 Hotstage to heat or cool the sample with a rate of $10^{\circ} \mathrm{C} / \mathrm{min}$. POM was used to determine melt-temperatures of samples which could not be measured via DSC (e.g. because of degradation upon melting). The melt-temperature or range was determined by visual aid of the samples becoming isotropic in nature. Matrix assisted laser desorption/ionization-time of flight-mass spectroscopy (MALDI-ToF-MS) analysis was recorded on a Voyager DESTR from Applied Biosystems (laser frequency $20 \mathrm{~Hz}, 337 \mathrm{~nm}$ and a voltage of $25 \mathrm{kV}$ ). The matrix material was DCTB $(40 \mathrm{mg} / \mathrm{mL}$ in HFIP). Potassium trifluoroacetate was added as cationic ionization agent $(5 \mathrm{mg} / \mathrm{mL}$ in HFIP). The polymer sample was dissolved in HFIP $(1 \mathrm{mg} / \mathrm{mL})$, to which the matrix material and the ionization agent were added $(5: 1: 5)$. If the samples were not fully dissolved, the mixture was filtered over a $0.2 \mu \mathrm{m}$ PTFE syringe filter prior to placing on the target plate.

\section{Synthesis methods}

\section{Synthesis of FDCA-Cl}

2,5-Furandicarboxylic acid $(16.5 \mathrm{~g}, 0.11 \mathrm{~mol})$ was loaded in a $100 \mathrm{~mL}$ 3-neck round-bottom flask, equipped with a N2-inlet and a condenser, and was purged 3 times with argon/vacuum cycles. Thionyl chloride (27.5 $\mathrm{mL}, 0.38 \mathrm{~mol})$ and DMF $(100 \mu \mathrm{L}$, catalytic) were added, and the reaction mixture was refluxed for 4 hours at $85{ }^{\circ} \mathrm{C}$, until the reaction mixture was a clear solution. A N2-flow over the reaction ensured the formed gasses to pass through a gas-washing bottle $(250 \mathrm{~mL} 0.2 \mathrm{M}$ aq. $\mathrm{NaOH})$ attached via the top of the condenser. After cooling, the excess thionyl chloride was removed in vacuo, the product was isolated in several recrystallization steps from toluene as white crystals $(15.2 \mathrm{~g}, 75 \%$ yield).

\section{General synthesis of trimer pre-polyesters}

A generally applicable synthesis method for the preparation of trimer pre-polyesters has been developed (Scheme 1-2). The syntheses were performed in anhydrous conditions. A solution of $9 \mathrm{mmol}$ diol, $7 \mathrm{mmol}$ pyridine, and catalytic amount of DMAP in $5 \mathrm{~mL}$ anhydrous THF was stirred in an ice bath in a $25 \mathrm{~mL}$ 2-neck round-bottom flask equipped with a condenser. At $0{ }^{\circ} \mathrm{C}$, under $\mathrm{N}_{2}$-flow, a solution of $3 \mathrm{mmol}$ di-acid chloride in $3 \mathrm{~mL}$ anhydrous THF was added dropwise. After stirring the reaction mixture overnight, the THF was removed in vacuo. The product was extracted with $\mathrm{CHCl}_{3}(3 \times 50 \mathrm{~mL})$ and washed $10 \mathrm{w} \%$ aq. $\mathrm{Cu}_{2} \mathrm{SO}_{3}$ (3x $50 \mathrm{~mL}$ ), and with $0.01 \mathrm{M}$ aq. $\mathrm{HCl}\left(3 \times 50 \mathrm{~mL}\right.$ ). The $\mathrm{CHCl}_{3}$ extract was dried with $\mathrm{MgSO}_{4}$, filtered, and reduced in vacuo. Generally, the extracts could be used as is, however some required further purification by precipitation from $\mathrm{CHCl}_{3}$ in ether. Typically the trimers from FDCA and terephthalic acid were obtained as white solids. Purity was confirmed by NMR and/or LC-MS. The pre-polyesters were mostly isolated as trimers, however also some pentamers and heptamers are present. ${ }^{1} \mathrm{H}-\mathrm{NMR}$ analysis of trimers with $1,3-$ CPdiol is shown in the supplementary section for this chapter. 


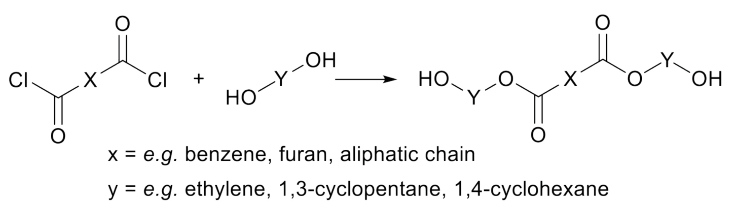

Scheme 1-2. General synthesis route to obtain trimer pre-polyesters.

\section{Trimer 1,2-ethyleneglycol-terephthalate (trimer PET)}

The product was obtained as white solids $\left(0.9 \mathrm{~g}, 72 \%\right.$ yield). ${ }^{1} \mathrm{H} \mathrm{NMR}\left(\mathrm{CDCl}_{3}, 300 \mathrm{MHz}\right): \delta 8.11$ (bs, $\left.4 \mathrm{H}\right)$, $4.95(\mathrm{~m}, 0.93 \mathrm{H}), 4.69(\mathrm{~m}, 2.11 \mathrm{H}), 4.31(\mathrm{~m}, 1.80 \mathrm{H}), 3.70(\mathrm{~m}, 1.81 \mathrm{H}) .{ }^{13} \mathrm{C} \mathrm{NMR}\left(\mathrm{CDCl}_{3}, 300 \mathrm{MHz}\right): \delta 165.5$ $(C=\mathrm{O}), 134.4 \& 133.79(\mathrm{O}=\mathrm{C}-\mathrm{C}), 130.1 \& 130.0(\mathrm{C}=\mathrm{C}), 67.5\left(\mathrm{HO}-\mathrm{CH}_{2}-\mathrm{CH}_{2}-\mathrm{O}-\mathrm{C}\right), 63.7\left(\mathrm{C}-\mathrm{O}-\mathrm{CH}_{2}-\mathrm{CH}_{2}-\mathrm{O}-\right.$ C), $59.4\left(\mathrm{HO}-\mathrm{CH}_{2}-\mathrm{CH}_{2}-\mathrm{O}-\mathrm{C}\right)$.

\section{Trimer 1,3-cyclopentanediol-terephthalate (CP-T)}

The product was obtained as white solids $\left(5.05 \mathrm{~g}, 76 \%\right.$ yield). ${ }^{1} \mathrm{H} \mathrm{NMR}\left(\mathrm{CDCl}_{3}, 300 \mathrm{MHz}\right): \delta 8.04(\mathrm{~s}, 4 \mathrm{H})$, 5.52 (bs, 0.28H), 5.39 (bs, 1.59H), 5.23 (bs, $0.23 \mathrm{H}), 4.67$ (bs, $1.77 \mathrm{H}), 2.8(\mathrm{~m}, 2.49 \mathrm{H}), 1.95(\mathrm{~m}, 5.43 \mathrm{H})$, $1.72(\mathrm{~m}, 2.23 \mathrm{H}), 1.55(\mathrm{~m}, 1.73 \mathrm{H}) .{ }^{13} \mathrm{C} \mathrm{NMR}\left(\mathrm{CDCl}_{3}, 300 \mathrm{MHz}\right): \delta 165.2(\mathrm{C}=\mathrm{O}), 77.2(\mathrm{CH}-\mathrm{OR}$ ring $) 70.8$ ( $\mathrm{CH}-\mathrm{OH}$ ring), $42.4\left(\mathrm{CH}_{2}\right.$ ring $), 33.6\left(\mathrm{CH}_{2}\right.$ ring $), 30.5\left(\mathrm{CH}_{2}\right.$ ring $)$.

\section{Trimer 1,3-cyclopentanediol-furanoate (CP-F)}

The product was obtained as white solids $\left(1.99 \mathrm{~g}, 62 \%\right.$ yield). ${ }^{1} \mathrm{H} \mathrm{NMR}\left(\mathrm{CDCl}_{3}, 300 \mathrm{MHz}\right): \delta 7.16(\mathrm{~m}, 2 \mathrm{H})$, $5.53(\mathrm{~m}, 1.90 \mathrm{H}), 5.39(\mathrm{~m}, 0.16 \mathrm{H}), 4.55(\mathrm{~m}, 1.30 \mathrm{H}), 4.40(\mathrm{~m}, 0.16 \mathrm{H}), 2.32(\mathrm{~m}, 2.59 \mathrm{H}), 2.13(\mathrm{~m}, 6.32 \mathrm{H}), 1.87$ $(\mathrm{m}, 2.27 \mathrm{H}), 1.70(\mathrm{~m}, 1.36 \mathrm{H}) .{ }^{13} \mathrm{C} \mathrm{NMR}\left(\mathrm{CDCl}_{3}, 300 \mathrm{MHz}\right): \delta 157.7(\mathrm{C}=\mathrm{O}), 146.7(\mathrm{C}-\mathrm{O}$, furan ring) $118.2(\mathrm{C}-$ $C$, furan ring) $77.4\left(\mathrm{CH}-\mathrm{OR}\right.$ ring) $72.1(\mathrm{CH}-\mathrm{OH}$ ring $), 42.2\left(\mathrm{CH}_{2}\right.$ ring $), 33.5\left(\mathrm{CH}_{2}\right.$ ring $), 30.3\left(\mathrm{CH}_{2}\right.$ ring $)$.

\section{Trimer 1,3-cyclopentanediol-adipate (CP-A)}

The product was obtained as orange oil $\left(5.31 \mathrm{~g}, 86 \%\right.$ yield). ${ }^{1} \mathrm{H}$ NMR $\left(\mathrm{CDCl}_{3}, 300 \mathrm{MHz}\right): \delta 5.27(\mathrm{~m}, 2.20 \mathrm{H})$, $5.17(\mathrm{~m}, 0.32 \mathrm{H}), 4.47(\mathrm{~m}, 1.75 \mathrm{H}), 4.33(\mathrm{~m}, 0.25 \mathrm{H}), 2.27(\mathrm{~m}, 7.73 \mathrm{H}), 1.97(\mathrm{~m}, 10.88 \mathrm{H}), 1.63(\mathrm{~m}, 9.52 \mathrm{H}) .{ }^{13} \mathrm{C}$ $\operatorname{NMR}\left(\mathrm{CDCl}_{3}, 300 \mathrm{MHz}\right): \delta 173.1(\mathrm{C}=\mathrm{O}), 75.5\left(\mathrm{CH}-\mathrm{OR}\right.$ ring) $72.3(\mathrm{CH}-\mathrm{OH}$ ring $), 42.4 \& 33.6 \& 30.5\left(\mathrm{CH}_{2}\right.$ ring), $34.1 \& 24.4\left(\mathrm{CH}_{2}\right.$ adipate $)$.

\section{Trimer 1,3-cyclopentanediol-sebacate (CP-S)}

The product was obtained light orange oil $(6.42 \mathrm{~g}, 88 \%$ yield $) .{ }^{1} \mathrm{H} \mathrm{NMR}\left(\mathrm{CDCl}_{3}, 300 \mathrm{MHz}\right): \delta 5.28(\mathrm{~m}$, 2.44H), $5.16(\mathrm{~m}, 0.31 \mathrm{H}), 4.47(\mathrm{~m}, 1.78 \mathrm{H}), 4.32(\mathrm{~m}, 0.22 \mathrm{H}), 2.24(\mathrm{~m}, 10.89 \mathrm{H}), 1.96(\mathrm{~m}, 8.12 \mathrm{H}), 1.59(\mathrm{~m}$, $10.97 \mathrm{H}), 1.29$ (bs, 11.89H). ${ }^{13} \mathrm{C} \mathrm{NMR}\left(\mathrm{CDCl}_{3}, 300 \mathrm{MHz}\right): \delta 173.6(\mathrm{C}=\mathrm{O}), 75.4(\mathrm{CH}-\mathrm{OR}$ ring $) 72.3(\mathrm{CH}-\mathrm{OH}$ ring), $42.4 \& 33.6$ \& $30.4\left(\mathrm{CH}_{2}\right.$ ring $), 34.5 \& 29.0 \& 24.9\left(\mathrm{CH}_{2}\right.$ sebacate $)$.

\section{Trimer 1,4-cyclohexanediol-terephthalate (CH-T)}

The product was obtained as white solids $(0.89 \mathrm{~g}, 50 \%$ yield $) .{ }^{1} \mathrm{H} \mathrm{NMR}\left(\mathrm{CDCl}_{3}, 300 \mathrm{MHz}\right): \delta 8.09(\mathrm{~m}, 4 \mathrm{H})$, 5.17 (bs, $1.24 \mathrm{H}), 5.04(\mathrm{bs} .076 \mathrm{H}), 3.83(\mathrm{~m}, 1.56 \mathrm{H}), 2.05(\mathrm{~m}, 6.09 \mathrm{H}), 1.71(\mathrm{~m}, 10.28 \mathrm{H}) .{ }^{13} \mathrm{C} \mathrm{NMR}\left(\mathrm{CDCl}_{3}\right.$, $300 \mathrm{MHz}$ ): $\delta 165.2(\mathrm{C}=\mathrm{O}$ ), 129.5 ( $\mathrm{C}=\mathrm{C}$ terephthalic ring), 72.7 (cis- $\mathrm{CH}-\mathrm{OR}$ ), 70.8 (trans- $\mathrm{CH}-\mathrm{OR}$ ), 68.7 \& $67.8(\mathrm{CH}-\mathrm{OH}), 32.0 \& 30.5 \& 28.3 \& 27.5\left(\mathrm{CH}_{2}\right.$ cyclohexane $)$. 


\section{Trimer 1,4-cyclohexanediol-adipate (CH-A)}

The product was obtained as a yellow oil $\left(1.78 \mathrm{~g}, 95 \%\right.$ yield). ${ }^{1} \mathrm{H} \mathrm{NMR}\left(\mathrm{CDCl}_{3}, 300 \mathrm{MHz}\right): \delta 4.74$ (ds,

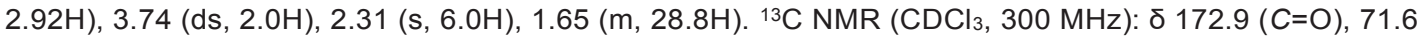
\& $69.7(\mathrm{CH}-\mathrm{OR}), 68.8$ \& $67.8(\mathrm{CH}-\mathrm{OH}), 34.1 \& 24.3\left(\mathrm{CH}_{2}\right.$ adipate $), 32.1 \& 30.5 \& 28.6 \& 27.4\left(\mathrm{CH}_{2}\right.$ cyclohexane).

\section{Trimer 1,4-cyclohexanediol-sebacate (CH-S)}

The product was obtained as white waxy solids (1.64 g, 98\% yield). ${ }^{1} \mathrm{H} \mathrm{NMR}\left(\mathrm{CDCl}_{3}, 300 \mathrm{MHz}\right): \delta 4.87$ (ds,

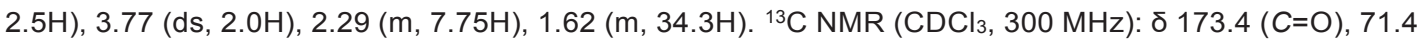
\& $69.4(\mathrm{CH}-\mathrm{OR}), 68.9 \& 67.9(\mathrm{CH}-\mathrm{OH}), 34.7 \& 25.0\left(\mathrm{CH}_{2}\right.$ adipate $), 32.2 \& 30.5 \& 29.0 \& 27.4\left(\mathrm{CH}_{2}\right.$ cyclohexane).

\section{Trimer 1,4-cyclohexanedimethanol-terephthalate (CHdm-T)}

The product was obtained as white solids $\left(1.36 \mathrm{~g}, 66 \%\right.$ yield). ${ }^{1} \mathrm{H} \mathrm{NMR}\left(\mathrm{CDCl}_{3}, 300 \mathrm{MHz}\right): \delta 8.10$ (bs, $6.06 \mathrm{H}), 4.29(\mathrm{ds}, 2.07 \mathrm{H}), 4.19(\mathrm{ds}, 3.70 \mathrm{H}), 3.56(\mathrm{ds}, 1.35 \mathrm{H}), 3.50(\mathrm{ds}, 2.64 \mathrm{H}), 1.90(\mathrm{~m}, 21.6 \mathrm{H}), 1.11(\mathrm{~m}$, $6.65 \mathrm{H}) .{ }^{13} \mathrm{C} \mathrm{NMR}\left(\mathrm{CDCl}_{3}, 300 \mathrm{MHz}\right): \delta 165.9(\mathrm{C}=\mathrm{O}), 134.2(\mathrm{O}=\mathrm{C}-\mathrm{C}$, terephthalic ring), $129.5(\mathrm{C}=\mathrm{C}$ terephthalic ring), $70.3 \& 68.0\left(\mathrm{CH}_{2}-\mathrm{OR}\right), 68.4 \& 66.1\left(\mathrm{CH}_{2}-\mathrm{OH}\right), 40.4+37.4(\mathrm{CH}-\mathrm{OH}), 37.4 \& 34.6(\mathrm{CH}-$ OR), $29.1 \& 28.7 \& 25.6 \& 25.1\left(\mathrm{CH}_{2}\right.$ cyclohexane ring).

\section{Trimer 1,4-cyclohexanedimethanol-adipate (CHdm-A)}

The product was obtained light yellow oil $\left(1.94 \mathrm{~g}, 89 \%\right.$ yield). ${ }^{1} \mathrm{H}$ NMR $\left(\mathrm{CDCl}_{3}, 300 \mathrm{MHz}\right): \delta 3.93$ (ds, $1.22 \mathrm{H}), 3.84(\mathrm{ds}, 2.49 \mathrm{H}), 3.48(\mathrm{ds}, 0.65 \mathrm{H}), 3.40(\mathrm{ds}, 1.35 \mathrm{H}), 2.26(\mathrm{bs}, 3.75 \mathrm{H}), 1.60(\mathrm{~m}, 15.85 \mathrm{H}), 0.93(\mathrm{~m}$, 3.93H). ${ }^{13} \mathrm{C} \mathrm{NMR}\left(\mathrm{CDCl}_{3}, 300 \mathrm{MHz}\right): \delta 173.4(\mathrm{C}=\mathrm{O}), 69.3 \& 67.1\left(\mathrm{CH}_{2}-\mathrm{OR}\right), 68.4 \& 66.0\left(\mathrm{CH}_{2}-\mathrm{OH}\right), 40.3+$ $37.3(\mathrm{CH}-\mathrm{OH}), 37.3 \& 34.5(\mathrm{CH}-\mathrm{OR}), 33.9 \& 24.4\left(\mathrm{CH}_{2}\right.$, adipate), $29.0 \& 28.8 \& 28.7 \& 25.5 \& 25.3 \& 25.1$ $\left(\mathrm{CH}_{2}\right.$ cyclohexane ring).

\section{Trimer 1,4-cyclohexanedimethanol-sebacate (CHdm-S)}

The product was obtained colorless oil $\left(1.74 \mathrm{~g}, 91 \%\right.$ yield). ${ }^{1} \mathrm{H} \mathrm{NMR}\left(\mathrm{CDCl}_{3}, 300 \mathrm{MHz}\right): \delta 3.98(\mathrm{ds}, 1.86 \mathrm{H})$, $3.90(\mathrm{ds}, 3.96 \mathrm{H}), 2.90(2.53 \mathrm{H}), 2.29(\mathrm{~m}, 6.78 \mathrm{H}), 1.82(\mathrm{bs}, 7.95 \mathrm{H}), 1.43(\mathrm{~m}, 31.10 \mathrm{H}), 1.00(\mathrm{~m}, 6.81 \mathrm{H}) .{ }^{13} \mathrm{C}$ $\operatorname{NMR}\left(\mathrm{CDCl}_{3}, 300 \mathrm{MHz}\right): \delta 174.0(\mathrm{C}=\mathrm{O}), 69.3 \& 67.0\left(\mathrm{CH}_{2}-\mathrm{OR}\right), 68.4 \& 66.1\left(\mathrm{CH}_{2}-\mathrm{OH}\right), 40.4+37.3(\mathrm{CH}-$ $\mathrm{OH}), 37.3 \& 34.3(\mathrm{CH}-\mathrm{OR}), 34.3 \& 25.1\left(\mathrm{CH}_{2}\right.$, sebacate), $29.0 \& 28.7 \& 25.5\left(\mathrm{CH}_{2}\right.$ cyclohexane ring).

\section{Polymerization screening methods}

\section{Small scale screening in TGA}

Trimer pre-polyesters were polymerized in the TGA with or without catalyst. Trimers with catalyst were prepared by dissolving the trimer in $\mathrm{CHCl}_{3}$ followed by the addition of $1 \mathrm{~mol} \%$ catalyst solution $(0.01 \mathrm{M}$ in anhydrous $\mathrm{CHCl}_{3}$ ). Next, the $\mathrm{CHCl}_{3}$ was removed in vacuo, and the trimers with catalyst were stored in a desiccator until use. Polymerization in the TGA was performed by loading 5-10 mg of trimer with or without catalyst in a TGA pan, and were heated with a rate of $10^{\circ} \mathrm{C} / \mathrm{min}$ to the desired polymerization temperature, followed by an isothermal for the desired polymerization time. The obtained polymers were cooled to room temperature and used as such for further analysis. 


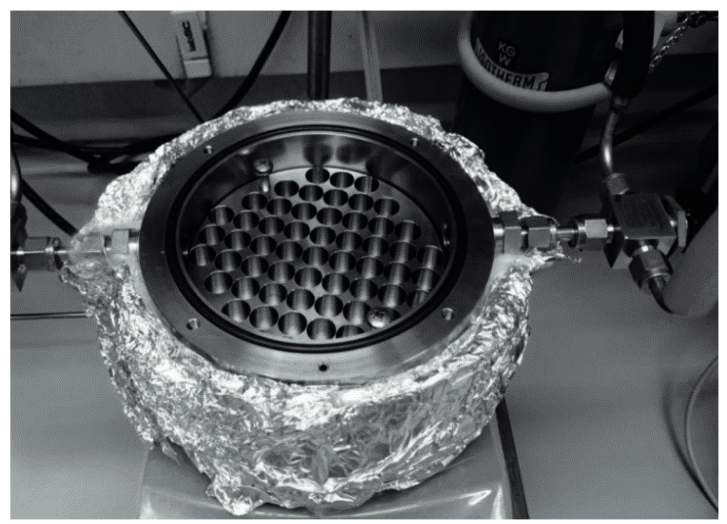

Figure 1-1. The image depicts the used stainless-steel reactor with a block for 57 HPLC vials for polycondensation screening reactions.

\section{Small scale screening reactor design}

The small-scale polycondensation reactions were carried out in a stainless-steel reactor. The design for this reactor (Figure 1-1) is based on earlier work of Gruter et al. ${ }^{11}$ : the stainless steel reactor block is designed to fit on top of a heating plate. To either side an inlet/outlet switch is attached for vacuum/ $\mathrm{N}_{2}$ control. On the inside is an interchangeable reactor block with space for up to 57 HPLC vials. Reactor temperatures were monitored via one temperature sensor in the bottom-middle of the reactor and via a temperature sensor located in the side of the reactor wall. The reactor lid is closed with 4 screws, and a rubber O-ring providing air-tight sealing. Due to the large mass of the reactor block heating from $25{ }^{\circ} \mathrm{C}$ to $200{ }^{\circ} \mathrm{C}$ is achieved in approximately 30 minutes. Temperature calibration experiments were performed using a temperature probe in oil inside the reactor and consistently showed a temperature in between the set temperature, and the temperature measured on the side as is shown in the supplementary section for this chapter.

The reactor is designed for thin-film polymerization reactions, as thin-film polycondensation reactions do not require stirring for effective condensate removal. Therefore the film thickness should be low enough so that diffusion and mass transfer do not limit the rate of polymerization. For the materials used in this study, placement of $4 \mathrm{mg}$ of polymer material in standard HPLC vial results in a thin-film with a thickness of roughly $200 \mu \mathrm{m}$ (taking into account an inner vial radius of $r=2.5 \mathrm{~mm}$ and assuming a polymer density of $\left.\rho=1.0 \mathrm{~g} / \mathrm{mm} 3\left(V=\left(\pi^{*} 2^{*} \mathrm{~h}\right) / \rho\right)\right)$.

\section{General small-scale screening reactor polymerization}

Trimer pre-polyesters were polymerized in the stainless-steel reactor block with or without catalyst. All the vials were weighed before and after reaction to monitor the weight loss during polymerization. Generally, $10 \pm 1 \mathrm{mg}$ of trimer was loaded in each vial, and $1 \mathrm{~mol} \%$ of catalyst solution $\left(0.01 \mathrm{M}\right.$ in anhydrous $\left.\mathrm{CHCl}_{3}\right)$ was added when required. The reactor block was placed in the reactor and next the reactor was closed after which it was purged $3 x$ with a $\mathrm{N}_{2}$ /vacuum cycle. The reactor was heated under a low $\mathrm{N}_{2}$-flow to the desired polymerization temperature, and kept at isothermal conditions for the desired polymerization time. Reduced pressure was applied during the polymerization when required. Polymerization was stopped by switching from vacuum to $\mathrm{N}_{2}$, followed by cooling of the reactor. After cooling down, the vials were weighed and the visible color and state of the polymer materials were noted. 


\section{Results and discussion}

\section{Trimer synthesis}

Initial thin-film polycondensation screening starting with a mixture of diols and dimethylesters of the desired trimers suffered from a high rate of evaporation and/or sublimation of the starting material. To overcome this problem, trimer pre-polyesters have been synthesized which are expected to be less volatile due to their higher mass and hence their higher boiling point. Following previous work of Sweileh et $\mathrm{al}^{23}$, a general synthesis method for the preparation of trimer pre-polyesters, shown in Scheme 1-2, was developed. The trimers are named by the combined abbreviations of the diol and the diacid: for examples the trimer of 1,3-CPdiol and 2,5-FDCA is abbreviated as CP-F. Endgroup analysis with ${ }^{1} \mathrm{H}-\mathrm{NMR}$ on trimer $\mathrm{CP}-\mathrm{F}$ (Figure 1-2, left) was obtained by subtracting the integral of end-group 6 of the integral of 2 , which leaves an integration of 0.47 protons. These extra protons of signal $\mathbf{2}$ come from non-end group CPdiol which can be found in higher oligomers such as pentamers $(n=2)$ and heptamers $(n=3)$. Since in higher oligomers $2 \mathrm{CH}$ contribute to signal 2, the ratio of heptamer is $0.47 / 2=0.235$ to 1 of trimer. LC-MS analysis (Figure 1-2, right) confirms the presence of higher oligomers in the form of pentamers and small amounts of heptamers. All trimers synthesized in this work were obtained with a small amount of higher oligomers, though the presence of higher oligomers is not expected to negatively affect the polymerization screening.
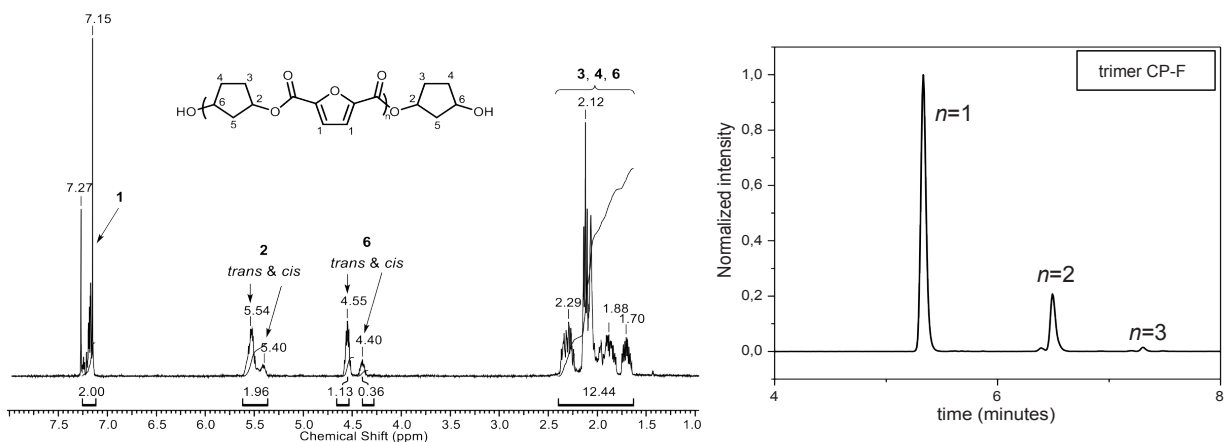

Figure 1-2. The image on the left depicts the ${ }^{1} \mathrm{H}-\mathrm{NMR}$ spectrum of CP-F trimer after synthesis. End-group analysis (signal 6 vs. signal 4) indicate a ratio of trimers vs. higher oligomers of 1 to 0.235 . LC-MS analysis (right) confirms the presence of higher oligomers in the form of pentamers and small amounts of heptamers.

Although the use of acid chlorides is not sustainable, it allows for synthesis of trimer prepolyesters, which is essential for the use in thin-film polycondensation conditions. However, for bulk polymerizations the use of acid chlorides is unnecessary as dimethyl esters and diols can readily be used together, since the rate of evaporation and sublimation is much lower. 


\section{Small-scale screening reactor design and validation}

The stainless steel reactor used for the small-scale polycondensation reactions (Figure 1-1) was first validated with the polycondensation of a well-known polymer; poly(ethylene terephthalate) (PET). The effect of film thickness, and thereby the use of this reactor a thin-film polycondensation reactor, was studied by loading the HPLC vials varying from $1 \mathrm{mg}$ to $100 \mathrm{mg}$ of PET trimer, with $1 \mathrm{~mol} \%$ of Ti(IV)butoxide as catalyst. The obtained molecular weights ( $M \mathrm{n}$, Figure 1-3 top, blue, and $Đ$, Figure 1-3 bottom, red)) showed a strong correlation with loading mass of trimers. With increasing monomer loading from 1 $\mathrm{mg}$ to $100 \mathrm{mg}$, the $M_{\mathrm{n}}$ values drops from $>25 \mathrm{~kg} / \mathrm{mol}$ to $<5 \mathrm{~kg} / \mathrm{mol}$, respectively. For a monomer loading around $10 \mathrm{mg}, M_{\mathrm{n}}$ values around $15 \mathrm{~kg} / \mathrm{mol}$ were consistently obtained on all runs. Therefore, to ensure repeatability of the polymerization experiments, in combination with ease in weighing, further work with this reactor is based on a monomer loading of $10 \pm 1 \mathrm{mg}$. A detailed overview of the polymerization reactions performed for assessment of the repeatability of the thin-film polymerization reactions is provided in the supplementary section for this chapter.

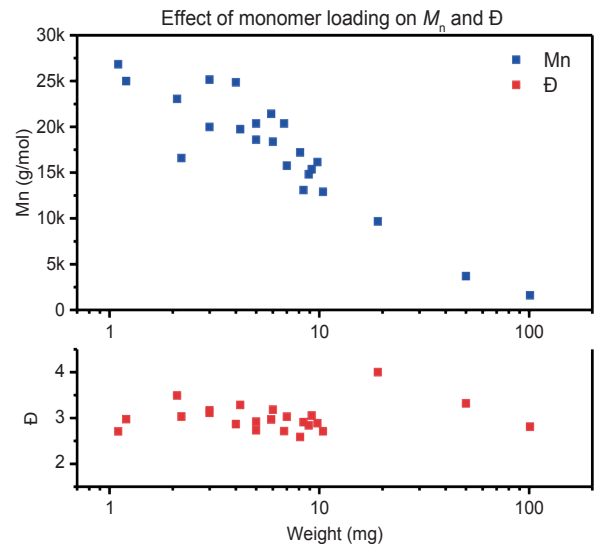

Figure 1-3. Overview of the effect of monomer loading (1-100 $\mathrm{mg}$ ) on $M_{\mathrm{n}}$ (top) and $Ð$ (bottom) for PET polymerized in the stainless steel reactor.

\section{Evaluation and optimization of polymerization conditions in TGA}

TGA studies were performed to ascertain the right polymerization conditions in terms of catalyst and temperature for each trimer. Therefore, TGA analysis was performed with a heating rate of $10{ }^{\circ} \mathrm{C} / \mathrm{min}$ from room temperature to $500{ }^{\circ} \mathrm{C}$ with and without catalyst. Figure 1-4 depicts the heating run of trimer CP-T (left) and CP-A (right) without catalyst (black), with $1 \mathrm{~mol} \% \mathrm{Ti}(\mathrm{IV})$ butoxide (red), and with $1 \mathrm{~mol} \% \mathrm{Sn}$ (II)octanoate (blue). It is clearly visible how both trimers without catalyst show a more classical s-shaped evaporation curve. Whereas the trimers mixed with a catalyst have an earlier onset of weight-loss. Some weight loss at lower temperatures could be the evaporation of remaining solvent $\left(\mathrm{CHCl}_{3}\right)$ from mixing the trimer with catalyst. However, the weight loss at higher temperatures have to be an effect of the added trans-esterification catalyst. Since no evaporation, sublimation, or degradation of the trimers occurs below $200{ }^{\circ} \mathrm{C}$, 
the increase in weight loss can be a sign of transesterification, where the removal of the condensate is observed as extra weight loss. Upon further heating the trimers also exhibit an higher thermal stability, which can be attributed to the formation of higher molecular weight oligomers or polymers. Furthermore, the weight-loss curves show a sort of "stable plateau", especially clear for trimer CP-A with Ti(IV)butoxide (Figure 1-4, right, red) as catalyst, which gives an indication for the optimal polymerization temperature.

To further study the optimal polycondensation temperatures, all trimers with $1 \mathrm{~mol} \%$ of
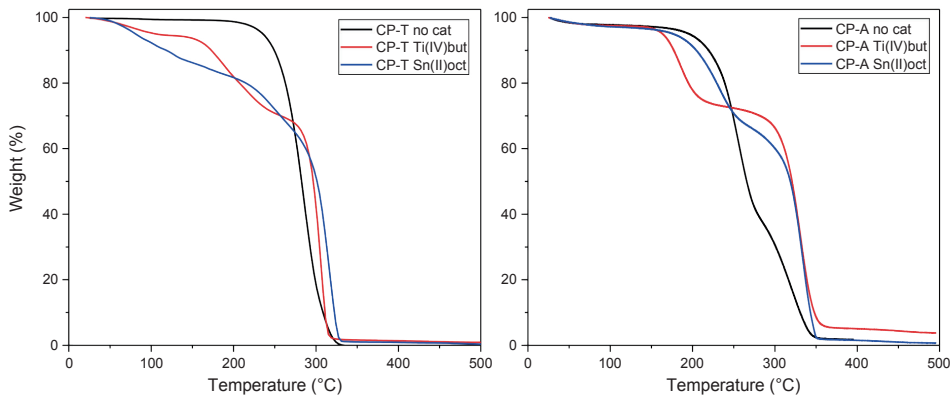

Figure 1-4. TGA studies of trimer CP-T (left) and CP-A (right) without catalyst (black), with $1 \mathrm{~mol} \%$ $\mathrm{Ti}\left(\mathrm{IV}\right.$ )butoxide (red), or $\mathrm{Sn}$ (II)octanoate (blue), with a standard heating rate of $10{ }^{\circ} \mathrm{C} / \mathrm{min}$ from room temperature to $500^{\circ} \mathrm{C}$. The effect of added catalyst is visible by the earlier onset of weight loss, and the subsequent higher heat stability.

catalyst were polymerized in the TGA with an 3-hour isothermal step at varying temperatures (e.g. 180, 210, and $240{ }^{\circ} \mathrm{C}$ ). Exemplified in Figure 1-5 is the temperature screening of all 1,3-CPdiol related trimers: CP-T (top left), CP-A (top right), CP-F (bottom left) and CP-S (bottom right). Most depicted trimers show a rapid onset in weight-loss after reaching the desired polycondensation temperature (15-19 minutes), and reach a near steady plateau for the remainder of the measurement. Most depicted samples run at lower temperatures (150 and $180{ }^{\circ} \mathrm{C}$ ) show a somewhat slow decrease of mass, with on average also a lower total \%weight loss. Additionally, all depicted samples run at 240 ${ }^{\circ} \mathrm{C}$ show a more rapid weight loss over time, suggesting thermal instability. Indeed, the obtained samples at temperatures at $210{ }^{\circ} \mathrm{C}$ or lower were mostly white or opaque in nature, whereas the obtained samples run at $240{ }^{\circ} \mathrm{C}$ were often discolored or even fully black.

Furthermore, the effect of catalyst on the polycondensation rate can be found by comparing the rate of rate loss at a set temperature. Figure 1-6 depicts the difference in weight loss of all 1,3-CPdiol related trimers: from bottom to top: CP-T, CP-A, CP-S, and $\mathrm{CP}-\mathrm{F}$ related to the catalyst used: either $\mathrm{Ti}(\mathrm{IV})$ butoxide (solid lines) or $\mathrm{Sn}$ (II)octanoate (dashed lines) at the polycondensation temperature of $210{ }^{\circ} \mathrm{C}$. It can clearly be seen that all trimers with $\mathrm{Ti}(\mathrm{IV})$ butoxide as catalyst show a rapid drop in weight loss upon reaching the polycondensation temperature ( 18 minutes). At the same time, all timers with $\mathrm{Sn}(\mathrm{II})$ octanoate depicts a more gradual weight loss over time. 

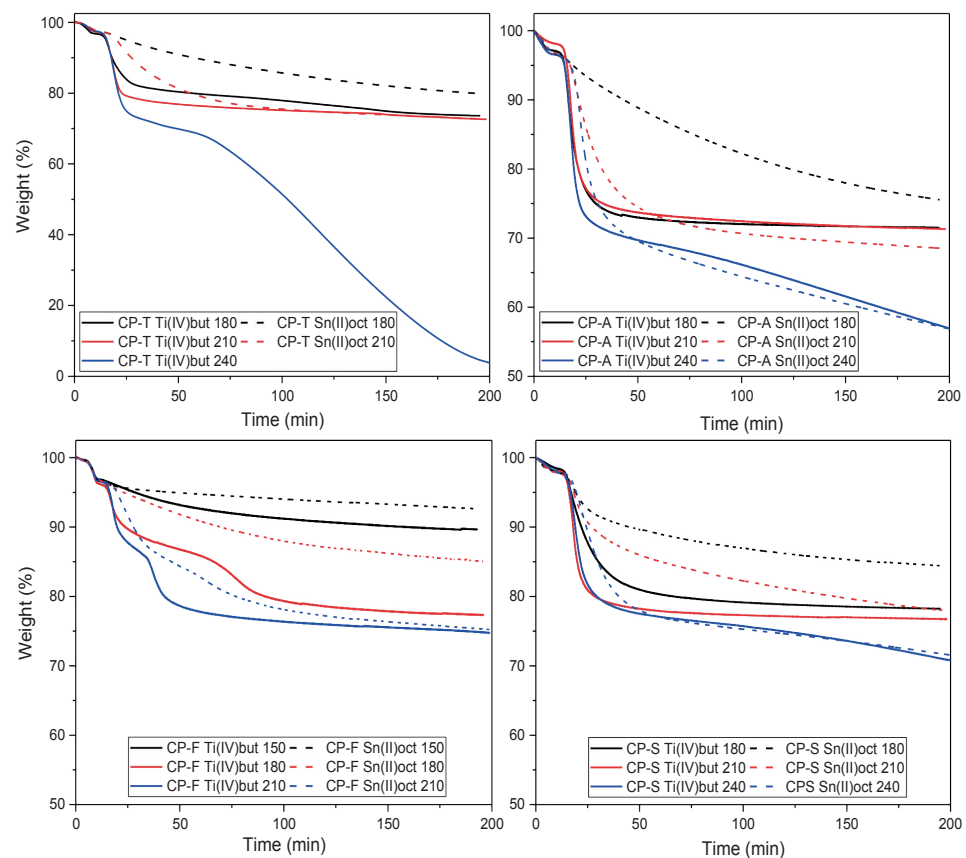

Figure 1-5. Isothermal TGA studies on trimer CP-T (top left), CP-A (top right), CP-F (bottom left), and CP-S (bottom right) with either $1 \mathrm{~mol} \%$ of Ti(IV)butoxide or SnlI)octanoate at different temperatures for 3 hours. The rate, and total, weight loss are indicators for the optimal polycondensation temperature per trimer.

The observed faster weight loss indicates that Ti(IV)butoxide is a more efficient catalyst for transesterification of these trimers under these conditions, and that this method could be used for further catalyst screening.

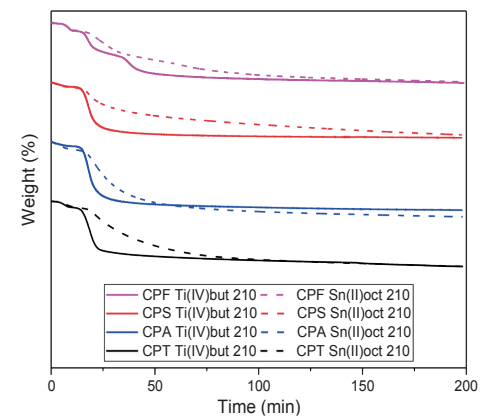

Figure 1-6. Isothermal TGA studies at $210^{\circ} \mathrm{C}$ on (from bottom to top) trimers CP-T, CP-A, CP-S, and CP-F with $1 \mathrm{~mol} \%$ of either Ti(IV)butoxide (solid line) or Sn(II)octanoate (dashed line). The rate of weight loss is an indicator for polycondensation efficiency per catalyst.

Since it is assumed that the mass-loss originates from the loss of a condensate unit, the total observed mass-loss at the optimal polycondensation temperatures could function as a measurement for the degree of polymerization. For example for trimer CP-F, the mass loss at the theoretical maximum conversion equals the mass-loss of one CPdiol 
group of the trimer, which contributes to $31 \%$ of the weight. The polycondensation conditions in the TGA are not considered to be ideal because it lacks a vacuum step, so reaching the theoretical maximum weight loss is not expected. Nevertheless, the \%weight loss can be used as an indicator for the polymerization rate, and thereby efficiency. Subsequent GPC analysis show a rise in molecular weight from trimers to higher molecular weight oligomers (on average $M_{\mathrm{n}}$ 400-2500, $M_{\mathrm{w}}$ 800-9000 g/mol), indicating that polycondensation in TGA is happening, however high molecular weight species are not formed due to the absence of a vacuum step. All trimers were analyzed for the weight loss, optical appearance, and molecular weight, and the data is summarized in Table 1-1. Furthermore, the optical appearance is a good indicator whether the related polymers will be semi-crystalline or not, and roughly what the $T_{\mathrm{g}}$ is for the amorphous polymers.

Table 1-1 Results of small-scale polycondensation reactions performed in TGA for all trimers at the established optimal temperature with $1 \mathrm{~mol} \%$ of $\mathrm{Ti}(\mathrm{IV})$ butoxide as catalyst.

\begin{tabular}{|c|c|c|c|c|c|c|}
\hline Trimer & $\begin{array}{l}\text { Optimal } \\
\text { pol. T }\end{array}$ & $\begin{array}{l}\text { Weight } \\
\text { loss }\end{array}$ & $\begin{array}{l}\text { Theoretical } \\
\text { weight loss }\end{array}$ & Color & $M_{\mathrm{n}}(\mathrm{g} / \mathrm{mol})$ & $M_{\mathrm{w}}(\mathrm{g} / \mathrm{mol})$ \\
\hline$C P-T$ & $210^{\circ} \mathrm{C}$ & $25.0 \%$ & $31 \%$ & white & 1390 & 4120 \\
\hline$C P-F$ & $210^{\circ} \mathrm{C}$ & $21.8 \%$ & $31 \%$ & off-white & n.d. & n.d. \\
\hline$C P-A$ & $180^{\circ} \mathrm{C}$ & $28.7 \%$ & $32 \%$ & transp. yellow & 1970 & 7240 \\
\hline$C P-S$ & $210^{\circ} \mathrm{C}$ & $21.1 \%$ & $28 \%$ & light brown & 980 & 2740 \\
\hline $\mathrm{CH}-\mathrm{T}$ & $230^{\circ} \mathrm{C}$ & $34.8 \%$ & $32 \%$ & light brown & 460 & 1060 \\
\hline $\mathrm{CH}-\mathrm{A}$ & $210^{\circ} \mathrm{C}$ & $29.7 \%$ & $34 \%$ & off-white & n.d. & n.d. \\
\hline $\mathrm{CH}-\mathrm{S}$ & $210^{\circ} \mathrm{C}$ & $24.8 \%$ & $29 \%$ & off-white & 1200 & 3290 \\
\hline$C H d m-T$ & $250^{\circ} \mathrm{C}$ & $24.6 \%$ & $34 \%$ & light brown & 1860 & 6900 \\
\hline$C H d m-A$ & $210^{\circ} \mathrm{C}$ & $24.8 \%$ & $36 \%$ & light yellow & n.d. & n.d. \\
\hline $\mathrm{CHdm}-\mathrm{S}$ & $210^{\circ} \mathrm{C}$ & $22.1 \%$ & $32 \%$ & off-white & 2070 & 5010 \\
\hline
\end{tabular}

\section{Assessment of 1,3-cyclopentanediol as building block}

The obtained optimal polycondensation conditions in terms of temperature and catalyst from the TGA screening discussed in the previous section could directly used for polycondensation reactions in the stainless steel reactor (Figure 1-1). Due to the ability to load 57 samples simultaneously, high-throughput experimentation could be realized for all different trimers. The addition of a vacuum step ensured both a more sterile environment (vacuum vs. TGA chamber) to limit side-reactions, and promoting the removal of the condensate to allow for build-up of higher molecular weight. Several polymerization runs can be found in the appendix of this chapter, providing the weight loss, color, molecular weight, and thermal properties of the obtained polymers.

The aforementioned method was used to assess the polymer properties of the new (potentially) biobased monomer 1,3-CPdiol, and the polymer properties were compared to commercially relevant diols $1,4-\mathrm{CHdiol}$ and $1,4-\mathrm{CHdmol}$. The polymers are 
abbreviated following the abbreviated of the trimer; i.e. the polymer of trimer CP-T is abbreviated as poly(CP-T). The obtained polymer properties are listed in Table 1-2.

Table 1-2. Overview of properties of prepared polymers of cycloaliphatic diols 1,3-cyclopentanediol (CP), 1,4cyclohexanediol $(\mathrm{CH})$, and 1,4-cyclohexanedimethanol $(\mathrm{CHdm})$, bridged by terephthalate $(\mathrm{T})$, adipate $(\mathrm{A})$, and sebacate (S) moieties. ${ }^{\text {a) }}$ Degradation upon melt, b) obtained via polarized optical microscopy.

\begin{tabular}{|c|c|c|c|c|}
\hline Polymer & $M_{\mathrm{n}}(\mathrm{g} / \mathrm{mol})$ & $M_{\mathrm{w}}(\mathrm{g} / \mathrm{mol})$ & $T_{g}\left({ }^{\circ} \mathrm{C}\right)$ & $T_{\mathrm{m}}\left({ }^{\circ} \mathrm{C}\right)$ \\
\hline poly $(C P-T)$ & $5 k$ & $11 \mathrm{k}$ & 65 & $240-300^{a}$ \\
\hline poly $(C P-F)$ & $5.6 \mathrm{k}$ & $11.1 \mathrm{k}$ & 70 & $240-300^{a}$ \\
\hline poly $(C P-A)$ & $17 \mathrm{k}$ & $57 \mathrm{k}$ & -30 & amorphous \\
\hline poly (CP-S) & $25 k$ & $92 k$ & -38 & 45 \\
\hline poly $(\mathrm{CH}-\mathrm{T})$ & $1.1 \mathrm{k}$ & $1.4 \mathrm{k}$ & n.o. & $300^{+b}$ \\
\hline poly $(\mathrm{CH}-\mathrm{A})$ & $13 k$ & $37 \mathrm{k}$ & 5 & 117 \\
\hline poly $(\mathrm{CH}-\mathrm{S})$ & $16 \mathrm{k}$ & $45 k$ & -22 & 75 \\
\hline poly $(\mathrm{CHdm}-\mathrm{T})$ & $4 \mathrm{k}$ & $7 \mathrm{k}$ & n.o. & $240^{+b}$ \\
\hline poly $(\mathrm{CHdm}-\mathrm{A})$ & $12 k$ & $27 \mathrm{k}$ & -30 & 92 \\
\hline poly $(\mathrm{CHdm}-\mathrm{S})$ & $18 \mathrm{k}$ & $42 k$ & -38 & 44 \\
\hline
\end{tabular}

Poly(CP-T) and poly(CP-F) were obtained as semi-crystalline polymers with an unstable melt between $240{ }^{\circ} \mathrm{C}$ and $300{ }^{\circ} \mathrm{C}$. Since the polymerization temperature is below the $T_{\mathrm{m}}$, a full melt-polycondensation is not achieved, leading to crystallization during polymerization. This crystallization can lead to shorter polymer chains, and the formation of small and large crystal domains, leading to a broad range for the first melt. However, an increase in temperature resulted in rapid degradation (observed by bubble-formation and discoloration), hence the polymerization temperature for these trimers was not raised, and the exact $T_{\mathrm{m}}$ could not be determined. Further comparison of polymers with $\mathrm{CPdiol}$ to the $\mathrm{CHdiol}$ and $\mathrm{CHdmol}$ counterparts shows that the thermal properties are very similar to those of $\mathrm{CHdmol}$, i.e. $T_{\mathrm{g}}$ poly $(\mathrm{CP}-\mathrm{A}) \approx \operatorname{poly}(\mathrm{CHdm}-\mathrm{A}) \approx-30{ }^{\circ} \mathrm{C}, T_{\mathrm{g}}$ poly $(\mathrm{CP}$ $\mathrm{S}) \approx \operatorname{poly}(\mathrm{CHdm}-\mathrm{S}) \approx-38^{\circ} \mathrm{C}$, and $T_{\mathrm{m}} \operatorname{poly}(\mathrm{CP}-\mathrm{S}) \approx \operatorname{poly}(\mathrm{CHdm}-\mathrm{S}) \approx 44^{\circ} \mathrm{C}$. The polymers with CPdiol compared to $\mathrm{CHdiol}$ are all lower in $T_{\mathrm{g}}$ and $T_{\mathrm{m}}$, indicating that polymers with $\mathrm{CHdiol}$ are more rigid, and exhibit a better crystal packing than polymers with CPdiol and CHdm. DSC analysis (Figure 1-7) shows that although the thermal transition temperatures of polymers with $\mathrm{CPdiol}$ are similar to those of $\mathrm{CHdmol}$, there are important differences. Poly (CP-A) is fully amorphous, whereas poly $(\mathrm{CHdm}-\mathrm{A})$ is semi-crystalline in nature (Figure 1-7, left), which is an indication that polymers with $\mathrm{CHdmol}$ exhibits better crystallization kinetics. 

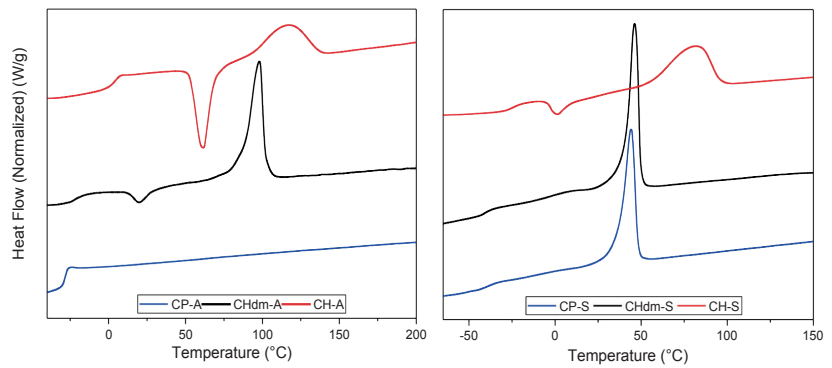

Figure 1-7. DSC curves of prepared polymers of cycloaliphatic diols 1,3-cyclopentanediol (CP, bottom), 1,4-cyclohexanedimethanol ( $\mathrm{CHdm}$, middle), and 1,4-cyclohexanediol $(\mathrm{CH}$, bottom) with soft blocks adipate (A, left) and sebacate (S, right). Heating and cooling performed with $10^{\circ} \mathrm{C} / \mathrm{min}, 2^{\text {nd }}$ heating run is shown.

Overall, these findings suggest that polymers containing CPdiol can be made, and will have similar thermal transition properties as polymers containing $\mathrm{CHdmol}$, albeit with somewhat hampered crystallization. In fact, under the reported conditions, $M \mathrm{n}$ values > $15 \mathrm{~kg} / \mathrm{mol}$ are readily obtained for polymers based on CPdiol and sebacic acid or adipic acid. However, the use of high-melting (>240 ${ }^{\circ} \mathrm{C}$ ) polymers with CPdiol should be avoided due to the observed unstable melt of poly(CP-F) and poly(CP-T), preventing melt processing of such polymers.

\section{Preparation of copolyesters}

Apart from preparing homo-polyesters, also copolyesters could readily be prepared via the aforementioned thin-film polycondensation in the parallel-reactor. As a proof of principle, trimers of CP-T and CP-F were mixed in three ratios (1:3, 1:1 and 3:1) weighed by molar ratio. The polymerization was performed with $1 \mathrm{~mol} \%$ of $\mathrm{Ti}(\mathrm{IV})$ butoxide at 220 ${ }^{\circ} \mathrm{C}$. All polymers were obtained as transparent glassy films, indicating that the furanoate and terephthalate domains cannot co-crystallize. The amorphous nature of these copolyesters could explain why a somewhat higher molecular weight could be obtained (Table 1-3) as compared to the homo-polyesters, since the polymerization temperature of $220^{\circ} \mathrm{C}$ is sufficient to keep everything in the molten state for better mixing and removal of the condensate. Though the broad dispersity caused by the presence of low molecular weight species indicates that the reaction time may need to be increased. DSC analysis was inconclusive to determine the $T_{\mathrm{g}}$, though manual probing of the copolymers during heating indicates the $T_{\mathrm{g}}$ to be between $60-80{ }^{\circ} \mathrm{C}$, as indicated by the copolymers to become soft.

Table 1-3. Obtained molecular weights for the copolymerization of trimer CP-T and CP-F with varying ratios.

\begin{tabular}{|c|c|c|c|c|}
\hline Polymer & Ratio T:F & $M_{\mathrm{n}}(\mathrm{g} / \mathrm{mol})$ & $M_{\mathrm{w}}(\mathrm{g} / \mathrm{mol})$ & $T_{m}\left({ }^{\circ} \mathrm{C}\right)$ \\
\hline poly $(C P-T-c o-F)$ & $3: 1$ & 3600 & 13000 & amorphous \\
\hline poly $(C P-T-c o-F)$ & $1: 1$ & 2600 & 17600 & amorphous \\
\hline $\operatorname{poly}(C P-T-c o-F)$ & $1: 3$ & 2800 & 14200 & amorphous \\
\hline
\end{tabular}


MALDI-ToF-MS analysis was used to determine the actual ratio of the copolymers. Figure 1-8, left, depicts the MALDI-ToF-MS spectrum of poly(CP-T-co-F) (1:1), where the distribution of repeating units $(n)$ can be seen according the degree of polymerization. It can be seen that each $n$ is not obtained as a single peak, but as a further distribution which is caused by the mass difference of 10 dalton between the repeat unit for CP-F $\left(M_{0}=220.2\right.$ dalton $)$ and CP-T $\left(M_{0}=232.2\right.$ dalton $)$.

In Figure 1-8, right, the zoomed in region of $n=9$ is shown, with the distribution for poly(CP-T-co-F) with the ratio $1: 3$ in blue (top), 1:1 in red (middle), and 3:1 in black (bottom). The dashed vertical lines represent the calculated masses for the possible copolymer ratios, which increases with steps of 10 dalton; ranging from 0 units of Terephthalate (T) and 9 units of Furanoate (F) on the left (at the 0 ), to 9 units of $T$ and 0 units of $\mathrm{F}$ at the right (at the 9). The peak intensity represents the distribution of the most common copolymer species, and the distribution curves form a near Gaussian distribution, showing that the copolymerization is truly random, as is expected for transesterification reactions. The highest peak represents the most common copolymer species, and can be taken as the obtained ratio for that specific copolymer. The highest peak for the 1:3 ratio (blue) resides at the dashed line 2, which indicates that 2 out of 9 acid groups are $\mathrm{T}$, and therefore 7 out of 9 acid groups are $\mathrm{F}$. This gives a ratio of $78 \% \mathrm{~F}$ $(7 / 9 * 100)$, which corresponds well to the added ratio of $75 \% \mathrm{~F}(3 /(1+3) * 100)$. Poly $(\mathrm{CP}-\mathrm{T}-$ co-F) $1: 1$ (blue) has the highest distribution at 5 (4 out of 9 acid groups are F), which corresponds to a ratio of $44 \% \mathrm{~F}$, which is close to the added ratio of $50 \% \mathrm{~F}$. And the ratio poly(CP-T-co-F) 3:1 (black) has the highest distribution at 6 (3 out of 9 acid groups are $\mathrm{F}$ ), which corresponds to a ratio of $33 \% \mathrm{~F}$, which is also close to the added ratio of $25 \% \mathrm{~F}$.

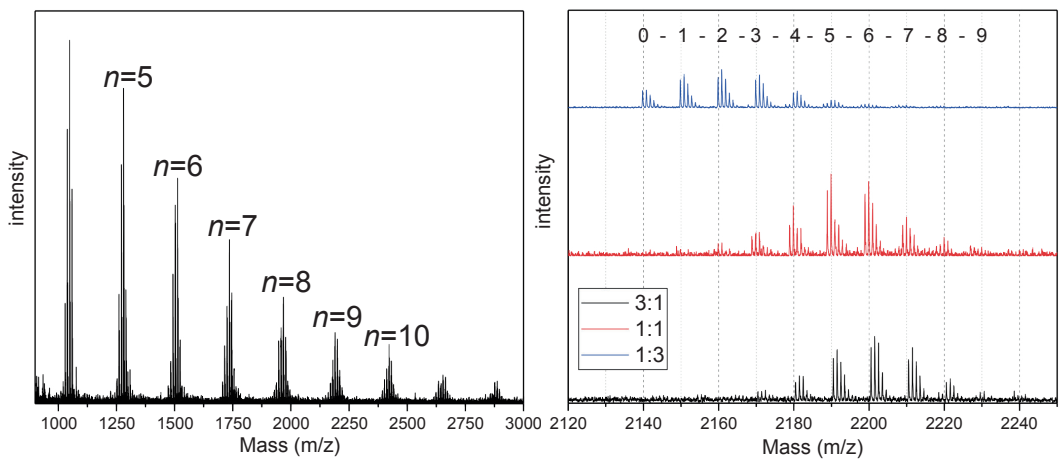

Figure 1-8. Left: MALDI-ToF-ms spectrum of poly(CP-T-co-F) with a 1:1 T:F ratio, the mass of the peaks corresponds to the amount of repeat units of the polymer, labelled $n$. Instead of a single peak + isotopes per repeat unit, a distribution of peaks is found according to the copolymer distribution. Right: Zoomed in area of $n=9$ of the copolymers with varying T:F ratio: top (blue) T:F 1:3, middle (red) T:F 1:1, and bottom (black) T:F 3:1. The vertical dashed lines represent the calculated masses for the copolymer with changing amounts of $\mathrm{T}$ and $\mathrm{F}$, with 0 units of $\mathrm{T}$ on the left, and 9 units of $\mathrm{T}$ on the right. The copolymer distribution is determined by the highest observed peaks. 


\section{Conclusions}

This work describes a novel small-scale screening method for polyesters with new biobased building blocks. With thin-film polycondensation reactions with $10 \mathrm{mg}$ starting, multiple reactions could readily be run which suits the limited availability of novel monomers from biomass. A generic synthesis route has been developed to prepare trimer pre-polyesters from various diols, in particular for 1,3-CPdiol, and di-acid-chloride monomers. These trimers were directly used in small-scale thin-film polycondensation reactions as they do not have the drawback of monomer evaporation. Using this approach, TGA was used as a screening technique to identify the thermal stability, and to determine optimal polymerization conditions in terms of temperature and catalyst for the CPdiol based trimers. Using the optimal polymerization temperature of $180-210{ }^{\circ} \mathrm{C}$, high molecular weight polymers (>15 kg/mol) with 1,3-CPdiol and sebacic acid or adipic acid were successfully made. A comparative study for thermal transition temperatures showed that polyesters having CPdiol exhibit lower $T_{\mathrm{g}}$ and $T_{\mathrm{m}}$ than their $\mathrm{CHdiol}$ counterpart, which can likely be attributed to the lower rigidity and crystallinity of CPdiol caused by the less stable half-boat conformation compared to the stable boatconformation of cyclohexane structures. Instead, the thermal transition temperatures for polyesters with CPdiol were found to be comparable to their $\mathrm{CHdmol}$ counterpart, albeit with slightly hampered crystallization, which is likely caused by the increased flexibility of $\mathrm{CHdmol}$ originating from the two extra methyl spacers.

\section{Acknowledgements}

This work was performed under the framework of Chemelot InSciTe and is supported by contributions from the European Regional Development Fund (ERDF) within the framework of OP-Zuid and with contributions from the province of Brabant and Limburg and the Dutch Ministry of Economy. 


\section{References}

1. Sara, M., Brar, S. K. \& Blais, J. F. Production of Drop-In and Novel Bio-Based Platform Chemicals. in Platform Chemical Biorefinery 249-283 (Elsevier, 2016).

2. Nakajima, H., Dijkstra, P. \& Loos, K. The Recent Developments in Biobased Polymers toward General and Engineering Applications: Polymers that are Upgraded from Biodegradable Polymers, Analogous to Petroleum-Derived Polymers, and Newly Developed. Polymers (Basel). 9, 523 (2017).

3. Hillmyer, M. A. The promise of plastics from plants. Science (80-. ). 358, 868-870 (2017).

4. Zhu, Y., Romain, C. \& Williams, C. K. Sustainable polymers from renewable resources. Nature 540, 354-362 (2016).

5. Bomtempo, J.-V., Chaves Alves, F. \& de Almeida Oroski, F. Developing new platform chemicals: what is required for a new bio-based molecule to become a platform chemical in the bioeconomy? Faraday Discuss. 202, $213-225$ (2017).

6. Hoogenboom, R. \& Schubert, U. S. High-throughput synthesis equipment applied to polymer research. Rev. Sci. Instrum. 76, 062202 (2005).

7. Meier, M. a R., Hoogenboom, R. \& Schubert, U. S. Combinatorial Methods, Automated Synthesis and High-Throughput Screening in Polymer Research: The Evolution Continues. Macromol. Rapid Commun. 25, 21-33 (2004).

8. Murphy, V. et al. High-Throughput Approaches for the Discovery and Optimization of New Olefin Polymerization Catalysts. Chem. Rec. 2, 278-289 (2002).

9. Potyrailo, R. A., Lemmon, J. P. \& Leib, T. K. High-Throughput Screening of Selectivity of Melt Polymerization Catalysts Using Fluorescence Spectroscopy and Two-Wavelength Fluorescence Imaging. Anal. Chem. 75, 4676-4681 (2003).

10. Brocchini, S., James, K., Tangpasuthadol, V. \& Kohn, J. Structure-property correlations in a combinatorial library of degradable biomaterials. J. Biomed. Mater. Res. 42, 66-75 (1998).

11. M. Gruter, G.-J., Sipos, L. \& Adrianus Dam, M. Accelerating Research into Bio-Based FDCA-Polyesters by Using Small Scale Parallel Film Reactors. Comb. Chem. High Throughput Screen. 15, 180-188 (2012).

12. Li, G. et al. Industrially scalable and cost-effective synthesis of 1,3-cyclopentanediol with furfuryl alcohol from lignocellulose. Green Chem. 18, 3607-3613 (2016).

13. Celli, A. et al. Effect of 1,4-cyclohexylene units on thermal properties of poly(1,4-cyclohexylenedimethylene adipate) and similar aliphatic polyesters. Polym. Int. 62, 1210-1217 (2013).

14. Kibler, C. J., Bell, A. \& Smith, J. G. Polyesters of 1,4-cyclohexanedimethanol1. J. Polym. Sci. Part A Gen. Pap. 2, 21152125 (1964).

15. Berti, C. et al. Environmentally Friendly Copolyesters Containing 1,4-Cyclohexane Dicarboxylate Units, 1-Relationships Between Chemical Structure and Thermal Properties. Macromol. Chem. Phys. 211, 1559-1571 (2010).

16. Zhang, M., Moore, R. B. \& Long, T. E. Melt transesterification and characterization of segmented block copolyesters containing 2,2,4,4-tetramethyl-1,3-cyclobutanediol. J. Polym. Sci. Part A Polym. Chem. 50, 3710-3718 (2012).

17. Kelsey, D. R., Scardino, B. M., Grebowicz, J. S. \& Chuah, H. H. High impact, amorphous terephthalate copolyesters of rigid 2,2,4,4-tetramethyl-1,3-cyclobutanediol with flexible diols. Macromolecules 33, 5810-5818 (2000).

18. Sandhya, T. E., Ramesh, C. \& Sivaram, S. Copolyesters Based on Poly(butylene terephthalate)s Containing Cyclohexyl and Cyclopentyl Ring: Effect of Molecular Structure on Thermal and Crystallization Behavior. Macromolecules 40, 69066915 (2007).

19. Abraham, R. J. \& Koniotou, R. Conformational analysis. Part 40: a theoretical and NMR investigation of the conformations ofcis- andtrans-cyclopentane-1,3-diol. Magn. Reson. Chem. 41, 1000-1008 (2003).

20. Mattson, A. et al. Kinetic Resolution of Chiral Auxiliaries with C2-Symmetry by Lipase-Catalyzed Alcoholysis and Aminolysis. Acta Chem. Scand. 50, 918-921 (1996).

21. Fransson, A.-B. L., Xu, Y., Leijondahl, K. \& Bäckvall, J.-E. Enzymatic Resolution, Desymmetrization, and Dynamic Kinetic Asymmetric Transformation of 1,3-Cycloalkanediols. J. Org. Chem. 71, 6309-6316 (2006).

22. Agou, T. et al. Synthesis of a new highly-fluorinated cis -1,2-cyclopentanediol and its application for fluorinated oligoesters. J. Fluor. Chem. 210, 78-82 (2018).

23. Sweileh, B. A., Al-Qalawi, H. R. \& Mohammad, H. A. Synthesis and characterization of poly(ester carbonate)s by meltphase interchange reactions of dihydroxy compounds with alkylene and arylene diphenyl dicarbonates containing ester groups. J. Appl. Polym. Sci. 131, 39904 (2014). 


\section{Supplementary information for Chapter 1}

${ }^{1} \mathrm{H}-\mathrm{NMR}$ spectra of synthesized trimers with 1,3-cyclopentanediol

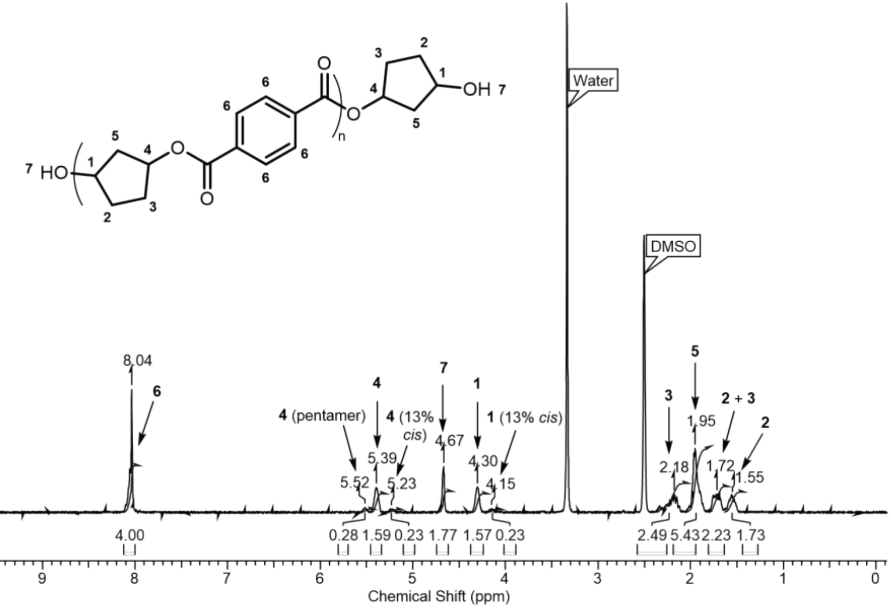

Figure S1 - 1. ${ }^{1} \mathrm{H}$-NMR analysis of trimer 1,3-cyclopentanediol-terephthalate.

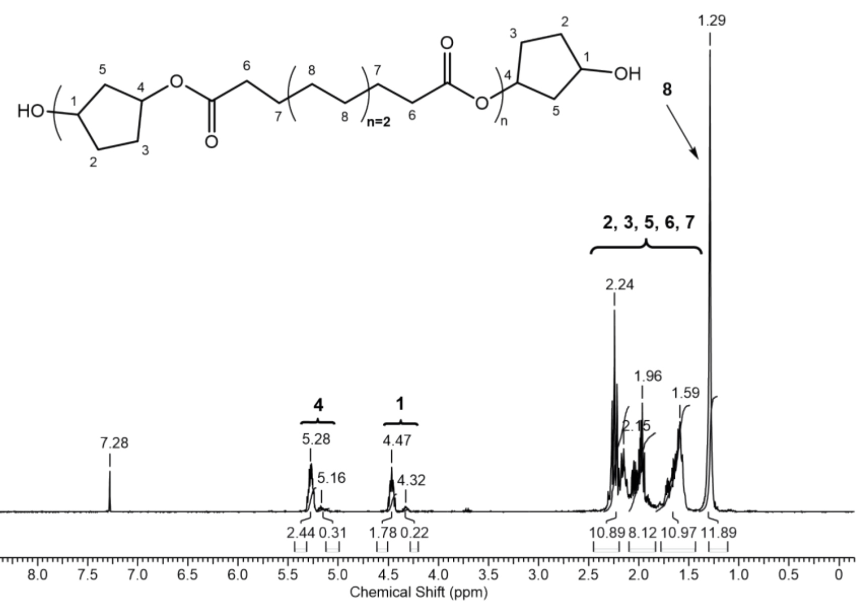

Figure $\mathrm{S} 1$ - 2. ${ }^{1} \mathrm{H}-\mathrm{NMR}$ analysis of trimer 1,3-cyclopentanediol-adipate. 


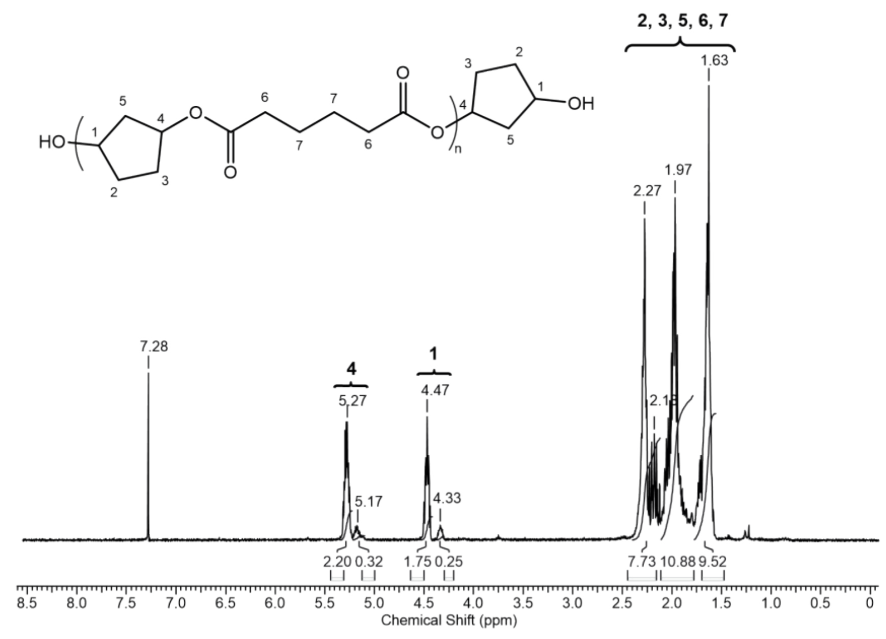

Figure S1 - 3. ${ }^{1} \mathrm{H}-\mathrm{NMR}$ analysis of trimer 1,3-cyclopentanediol-sebacate.

\section{Reactor block design}

The temperature consistency in the reactor was tested by measuring the temperature on 3 different points in the reactor: temperature sensor in the bottom part, in the side part, and inside in oil in a HPLC vial. There was consistently a small deviation in temperature inside the reactor as compared to the set $\&$ bottom temperature, which is likely caused by heat loss of the reactor itself to the surrounding environment. This temperature-loss was compensated for in the settings: i.e. for a desired polycondensation temperature of $220^{\circ} \mathrm{C}$, the temperature was set to $228^{\circ} \mathrm{C}$.

Table S1 - 1. Obtained values for temperature control throughout the stainless steel reactor.

\begin{tabular}{c|c|c|c|} 
Set temp ( $\left.{ }^{\circ} \mathrm{C}\right)$ & Control bottom & Control side & Oil in vials \\
\hline $100{ }^{\circ} \mathrm{C}$ & $98^{\circ} \mathrm{C}$ & $92^{\circ} \mathrm{C}$ & $92^{\circ} \mathrm{C}$ \\
\cline { 2 - 4 } $150^{\circ} \mathrm{C}$ & $148^{\circ} \mathrm{C}$ & $140^{\circ} \mathrm{C}$ & $142^{\circ} \mathrm{C}$ \\
\cline { 2 - 4 } $200^{\circ} \mathrm{C}$ & $198^{\circ} \mathrm{C}$ & $186^{\circ} \mathrm{C}$ & $188^{\circ} \mathrm{C}$ \\
\cline { 2 - 4 } $250^{\circ} \mathrm{C}$ & $246{ }^{\circ} \mathrm{C}$ & $230^{\circ} \mathrm{C}$ & $237^{\circ} \mathrm{C}$ \\
\cline { 2 - 4 } & &
\end{tabular}

The reactor was validated for polycondensation experiments by the polymerization of a known polymer: poly(ethylene terephthalate). First a consistency check was performed by polymerizing batches of $10 \mathrm{mg}$ over 4 runs (Table S1 - 2), and secondly the effect of loading on the polycondensation was checked (Table S1 - 3). Overall, the polycondensation was considered to perform consistently well in order to perform polycondensation reactions on the novel polyesters described in this work. 


\section{Detailed polymerization of small-scale screening}

Table S1 - 2. Details of polymerization of trimer ethylene glycol-terephthalate (E-T) for validation of consistency of small-scale reactor over multiple runs. Weight input is in milligrams.

\begin{tabular}{|c|c|c|c|c|c|c|c|c|c|c|c|c|c|c|c|c|c|}
\hline ه & $\begin{array}{l}0 \\
\infty \\
0\end{array}$ & $\underset{\mathscr{\leftarrow}}{\mathscr{\leftarrow}}$ & 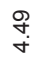 & $\stackrel{\leftrightarrow}{\forall}$ & $\stackrel{\stackrel{9}{\leftarrow}}{+}$ & 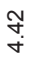 & 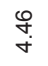 & $\begin{array}{l}\stackrel{0}{N} \\
\text { in }\end{array}$ & $\stackrel{\hat{o}}{\dot{r}}$ & م & $\stackrel{\Sigma}{i}$ & $\underset{\stackrel{\mathscr{D}}{N}}{\stackrel{\mathscr{D}}{ }}$ & $\begin{array}{l}\mathscr{\infty} \\
\stackrel{\infty}{\infty}\end{array}$ & $\begin{array}{l}\infty \\
\infty \\
\infty\end{array}$ & $\stackrel{\infty}{\sim}$ & $\stackrel{\leftrightarrow}{\stackrel{\leftrightarrow}{N}}$ & $\hat{i}$ \\
\hline 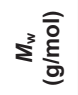 & $\begin{array}{l}8 \\
0 \\
0 \\
6\end{array}$ & $\begin{array}{l}\text { ¿ } \\
\stackrel{0}{\circ}\end{array}$ & 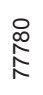 & $\begin{array}{l}\text { ठ্. } \\
\text { ه্口内 }\end{array}$ & 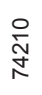 & 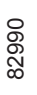 & 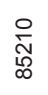 & $\begin{array}{l}\stackrel{8}{\infty} \\
\frac{\infty}{\infty}\end{array}$ & $\begin{array}{l}\text { : } \\
\text { \& } \\
\triangleright\end{array}$ & 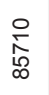 & $\begin{array}{l}\text { P } \\
\text { ơ }\end{array}$ & 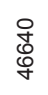 & $\frac{8}{i}$ & $\frac{8}{i}$ & $\stackrel{\circ}{\stackrel{N}{̃ ் ~}}$ & $\begin{array}{l}\text { D } \\
\underset{\sim}{N}\end{array}$ & $\stackrel{8}{\stackrel{8}{ }}$ \\
\hline 乏을 & 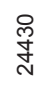 & $\begin{array}{l}\stackrel{0}{\infty} \\
\stackrel{N}{N}\end{array}$ & 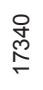 & $\begin{array}{l}\stackrel{\circ}{\circ} \\
\stackrel{\leftrightarrow}{\circ}\end{array}$ & 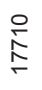 & 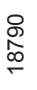 & 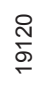 & 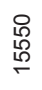 & $\begin{array}{l}\stackrel{\circ}{0} \\
\stackrel{0}{\circ} \\
\stackrel{\circ}{\leftarrow}\end{array}$ & 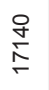 & ষ্ণ & $\stackrel{\circ}{\circ}$ & 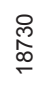 & 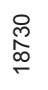 & స్ి & 点 & 웅 \\
\hline$\frac{\grave{0}}{0}$ & $\frac{\Phi}{\frac{\pi}{3}}$ & 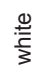 & $\frac{\stackrel{0}{\frac{1}{3}}}{\frac{1}{3}}$ & 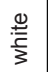 & $\frac{\stackrel{0}{\frac{\pi}{3}}}{\frac{1}{3}}$ & $\frac{9}{\frac{\underline{\pi}}{3}}$ & $\stackrel{\frac{0}{5}}{\frac{ \pm}{3}}$ & $\stackrel{\frac{0}{2}}{\frac{7}{3}}$ & 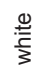 & $\frac{\stackrel{0}{\frac{\pi}{3}}}{\frac{\pi}{3}}$ & $\frac{0}{\frac{ \pm}{3}}$ & $\stackrel{\frac{0}{5}}{\frac{5}{3}}$ & 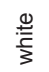 & $\frac{\stackrel{4}{\frac{\pi}{3}}}{\frac{3}{3}}$ & $\frac{9}{\frac{2}{3}}$ & $\frac{\Phi}{\frac{ \pm}{3}}$ & $\frac{\stackrel{0}{\frac{1}{3}}}{\frac{1}{3}}$ \\
\hline 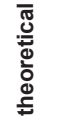 & $\stackrel{\stackrel{̊}{\sim}}{ }$ & $\stackrel{\stackrel{\circ}{\sim}}{\circ}$ & $\stackrel{\stackrel{\circ}{\sim}}{\text { d }}$ & $\stackrel{\circ}{\stackrel{े}{\sim}}$ & $\stackrel{\stackrel{े}{\sim}}{\text { d }}$ & ১ัণ & $\stackrel{\stackrel{̊}{\sim}}{ }$ & $\stackrel{\stackrel{े}{d ~}}{\circ}$ & $\stackrel{\stackrel{̊}{\sim}}{ }$ & $\stackrel{\stackrel{\circ}{\sim}}{\text { d }}$ & よे & $\stackrel{\stackrel{े}{d}}{ }$ & $\stackrel{\stackrel{̊}{\sim ~}}{ }$ & $\stackrel{\stackrel{\circ}{\sim}}{\mathrm{d}}$ & $\stackrel{\stackrel{\circ}{\sim}}{\sim}$ & $\stackrel{\stackrel{\circ}{\sim}}{\sim}$ & $\stackrel{\stackrel{\circ}{\sim}}{\text { d }}$ \\
\hline 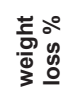 & 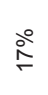 & ळें & ঃें & ஓें & ১ें & ๖े & ̊ें & 운 & 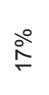 & 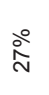 & @̊ & ○ें & $\stackrel{\circ}{\circ}$ & $\stackrel{\circ}{\stackrel{\circ}{\infty}}$ & & & \\
\hline 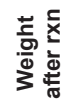 & เ & 0 & $\infty$ & 0 & $\wedge$ & 0 & $\infty$ & $\digamma$ & $\stackrel{\circ}{\circ}$ & $\infty$ & $\stackrel{\curvearrowright}{\curvearrowright}$ & 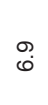 & $\sigma$ & $\sigma$ & & & \\
\hline 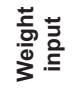 & 0 & $\sigma$ & $\stackrel{\circ}{\sim}$ & $\sigma$ & $\stackrel{\circ}{\sim}$ & $\infty$ & 우 & $\stackrel{m}{\sim}$ & $\stackrel{\simeq}{\simeq}$ & $\mp$ & \begin{tabular}{l}
\multirow{2}{*}{} \\
$\stackrel{0}{\circ}$
\end{tabular} & $\stackrel{\infty}{\infty}$ & $F$ & $\mp$ & $\begin{array}{l}\stackrel{L}{0} \\
\stackrel{0}{0}\end{array}$ & $\begin{array}{l}\stackrel{\circ}{\circ} \\
\stackrel{\circ}{\circ}\end{array}$ & $\check{\circ}$ \\
\hline 苋高 & ○ें & ஃ̊ํํ & ठั̊ & 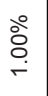 & ठั̊ & ठ̊ํ․ & ঃ̊̊ & ○ें & ○ें & $\begin{array}{l}\stackrel{\circ}{\circ} \\
\stackrel{\circ}{\circ}\end{array}$ & ذ̊̊̊̊口 & ○े & ○ें & ○ें & ○̊ํ․ & $\stackrel{\circ}{\circ}$ & ठั̊ \\
\hline Uే & $\sum_{i=\mid J}^{\stackrel{J}{\rho}}$ & $\sum_{i=}^{\stackrel{J}{\rho}}$ & 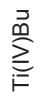 & 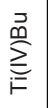 & $\sum_{i=}^{\stackrel{\jmath}{m}}$ & $\sum_{i=}^{\mathcal{M}}$ & 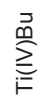 & $\sum_{i=}^{\mathcal{\rho}}$ & $\sum_{i=}^{\stackrel{J}{m}}$ & 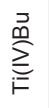 & 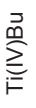 & $\sum_{i=}^{\mathcal{M}}$ & $\sum_{i=}^{\stackrel{g}{m}}$ & $\sum_{i=}^{\stackrel{p}{m}}$ & $\sum_{i=}^{\not{p}}$ & $\sum_{i=}^{\vec{m}}$ & $\sum_{i=}^{\mathcal{M}}$ \\
\hline 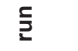 & - & - & - & - & - & - & - & - & - & - & $N$ & $N$ & $\sim$ & $m$ & $\sigma$ & 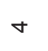 & $\nabla$ \\
\hline 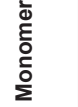 & 卢 & 占 & 它 & 占 & 它 & 岀 & 岀 & 占 & 占 & 它 & 岀 & เૅ & 占 & 岀 & 它 & 它 & 它 \\
\hline
\end{tabular}


Table S1 - 3. Details of polymerization of trimer ethylene glycol-terephthalate (E-T) for study of effect on monomer loading in small-scale reactor. Weight is in milligrams.

\begin{tabular}{|c|c|c|c|c|c|c|c|c|c|c|c|c|c|c|c|c|c|c|c|c|c|c|c|c|}
\hline ه & $\stackrel{\widehat{S}}{\mathrm{~N}}$ & $\underset{i}{i}$ & 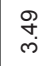 & 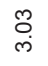 & $\stackrel{\hat{m}}{\dot{m}}$ & $\underset{m}{\check{m}}$ & 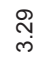 & $\begin{array}{c}\hat{\infty} \\
\stackrel{N}{N}\end{array}$ & 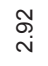 & $\stackrel{\mathcal{M}}{\stackrel{M}{N}}$ & $\stackrel{\infty}{\dot{m}}$ & बे. & $\begin{array}{l}m \\
\dot{m}\end{array}$ & $\stackrel{\Gamma}{i}$ & $\stackrel{\bar{N}}{\mathrm{~N}}$ & $\stackrel{\stackrel{R}{0}}{\mathrm{~N}}$ & 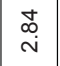 & $\begin{array}{l}\stackrel{0}{0} \\
\ddot{m}\end{array}$ & $\stackrel{\Gamma}{N}$ & $\begin{array}{l}\stackrel{\infty}{\infty} \\
\stackrel{\sim}{N}\end{array}$ & $\begin{array}{l}\infty \\
\infty \\
\infty\end{array}$ & $\nabla$ & $\underset{\widetilde{m}}{\widetilde{\rho}}$ & $\underset{\infty}{i}$ \\
\hline 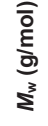 & $\stackrel{\circ}{\stackrel{9}{N}}$ & $\begin{array}{l}0 \\
\stackrel{0}{N} \\
\stackrel{N}{N}\end{array}$ & $\begin{array}{l}0 \\
\text { 㝵 } \\
\text { Oे }\end{array}$ & 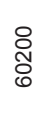 & 尺̊ & 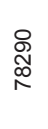 & 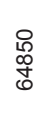 & $\frac{O}{\stackrel{N}{N}}$ & $\begin{array}{l}\stackrel{P}{Y} \\
\text { Hิ }\end{array}$ & $\begin{array}{l}\text { 呆 } \\
\text { 号 } \\
\text { in }\end{array}$ & $\begin{array}{c}P \\
\stackrel{0}{0} \\
i \\
i\end{array}$ & $\begin{array}{l}8 \\
0 \\
o \\
\varnothing\end{array}$ & $\begin{array}{l}0 \\
\stackrel{R}{f} \\
f\end{array}$ & 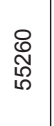 & 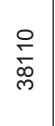 & \begin{tabular}{l}
8 \\
$\stackrel{8}{+}$ \\
\multirow{7}{*}{}
\end{tabular} & 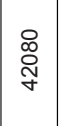 & $\begin{array}{l}\circ \\
\stackrel{\circ}{\circ} \\
\stackrel{\sigma}{\sigma}\end{array}$ & 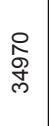 & 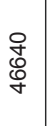 & 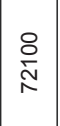 & $\begin{array}{l}\stackrel{8}{0} \\
\stackrel{0}{m}\end{array}$ & 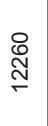 & 号 \\
\hline $\begin{array}{l}\widehat{\bar{े}} \\
\text { 을 } \\
\text { s }\end{array}$ & 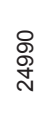 & $\begin{array}{l}\text { D } \\
\infty \\
\stackrel{N}{0}\end{array}$ & 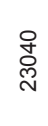 & 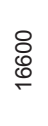 & $\begin{array}{l}\stackrel{\circ}{\circ} \\
\stackrel{\circ}{\circ}\end{array}$ & $\stackrel{\circ}{\stackrel{\circ}{\circ}}$ & $\begin{array}{l}\text { 京 } \\
\text { م }\end{array}$ & $\begin{array}{l}\stackrel{\circ}{\circ} \\
\stackrel{\circ}{\sim} \\
\stackrel{1}{2}\end{array}$ & $\begin{array}{l}\stackrel{8}{\circ} \\
\stackrel{\circ}{\infty} \\
\sim\end{array}$ & 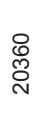 & 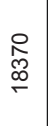 & 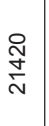 & $\begin{array}{c}8 \\
0 \\
5 \\
⺊\end{array}$ & 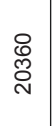 & $\begin{array}{l}\stackrel{8}{\circ} \\
\stackrel{p}{-}\end{array}$ & 옳 & 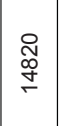 & $\begin{array}{l}8 \\
0 \\
{[0} \\
\end{array}$ & $\begin{array}{l}\text { \& } \\
\stackrel{N}{\sim}\end{array}$ & $\frac{8}{6}$ & $\mid \begin{array}{c}0 \\
\tilde{N} \\
\infty \\
\stackrel{0}{\infty}\end{array}$ & $\begin{array}{l}\stackrel{R}{8} \\
\stackrel{8}{8}\end{array}$ & $\begin{array}{l}\stackrel{8}{8} \\
\text { }\end{array}$ & 응 \\
\hline$\frac{\grave{0}}{\mathrm{~d}}$ & 造 & 造 & $\stackrel{\frac{0}{3}}{\frac{3}{3}}$ & $\stackrel{\frac{9}{2}}{\frac{\pi}{3}}$ & 造 & $\stackrel{\frac{9}{5}}{\frac{3}{3}}$ & $\stackrel{\frac{9}{3}}{\frac{3}{3}}$ & 造 & $\stackrel{\frac{9}{3}}{\frac{3}{3}}$ & $\frac{0}{\frac{\pi}{3}}$ & $\stackrel{\frac{9}{5}}{\frac{\pi}{3}}$ & 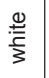 & $\stackrel{\frac{0}{5}}{\frac{\pi}{3}}$ & $\stackrel{\frac{9}{5}}{\frac{2}{3}}$ & $\frac{0}{\frac{2}{3}}$ & 䇋 & $\frac{\mathscr{0}}{\frac{7}{3}}$ & $\stackrel{\frac{9}{\frac{3}{3}}}{\frac{3}{3}}$ & 产 & $\stackrel{\frac{9}{3}}{\frac{1}{3}}$ & 造 & 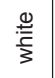 & $\frac{0}{\frac{2}{3}}$ & 黄 \\
\hline 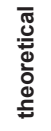 & $\stackrel{\stackrel{\leftrightarrow}{~}}{ }$ & $\stackrel{\stackrel{\leftrightarrow}{~}}{ }$ & $\stackrel{\stackrel{\circ}{\sim}}{\stackrel{0}{*}}$ & $\stackrel{\stackrel{\circ}{~}}{ }$ & $\stackrel{\stackrel{ }{~}}{\text { do }}$ & $\stackrel{\stackrel{̊}{\sim}}{ }$ & $\stackrel{\text { ণ }}{\text { Ò }}$ & $\stackrel{\text { ১̀ }}{\text { do }}$ & $\stackrel{\text { ণ }}{\text { do }}$ & $\stackrel{\stackrel{ }{~}}{ }$ & $\stackrel{\circ}{\stackrel{\sim}{~}}$ & $\stackrel{\circ}{\stackrel{ }{~}}$ & $\stackrel{\text { ̀ }}{\text { d }}$ & $\stackrel{\circ}{\stackrel{d}{~}}$ & 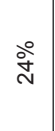 & $\stackrel{\circ}{\stackrel{\circ}{~}}$ & $\stackrel{\stackrel{\circ}{~}}{ }$ & $\stackrel{\stackrel{\circ}{\sim}}{\mathrm{O}}$ & $\stackrel{\circ}{\stackrel{\sim}{~}}$ & ঐ̊ & $\stackrel{\stackrel{\circ}{\sim}}{\sim}$ & $\stackrel{\stackrel{\Im}{\sim ~}}{\circ}$ & $\stackrel{\text { ১̊ }}{\mathrm{d}}$ & $\stackrel{\stackrel{\circ}{\sim}}{\stackrel{\circ}{*}}$ \\
\hline 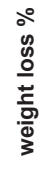 & 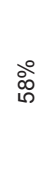 & 命 & 今े & $\stackrel{\stackrel{\circ}{N}}{\stackrel{1}{N}}$ & ळ్లి & ळे & 商 & 峁 & ஓें & ১ें & 今े & ڤ̊ & @̊̀ & ڤ్లి & ১ें & ¿े & ㅇํํ & $\stackrel{\stackrel{\leftrightarrow}{\sim}}{\mathrm{d}}$ & ¿̊ & 今े & $\stackrel{\circ}{\stackrel{\circ}{\circ}}$ & $\stackrel{\circ}{\circ}$ & 今े & ᄋे \\
\hline 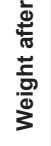 & $\stackrel{\mathscr{L}}{0}$ & L & $\stackrel{t}{r}$ & $\stackrel{0}{\longrightarrow}$ & $N$ & $N$ & $\hat{i}$ & $\stackrel{\leftrightarrow}{\sim}$ & $\stackrel{m}{m}$ & $\stackrel{m}{m}$ & $\stackrel{\leftrightarrow}{\dot{m}}$ & $\stackrel{m}{+}$ & กี & $\begin{array}{l}\mathscr{\bullet} \\
\dot{\leftarrow}\end{array}$ & $\stackrel{\vec{p}}{\mathrm{~b}}$ & 0 & $\hat{\varphi}$ & $\wedge$ & $\stackrel{\hat{N}}{ }$ & తి & $\infty$ & $\stackrel{\oplus}{\circ}$ & q & $\bar{\infty}$ \\
\hline 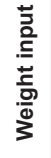 & $\stackrel{\sim}{=}$ & $\check{r}$ & $\bar{i}$ & $\stackrel{N}{N}$ & $m$ & $m$ & $\underset{+}{\stackrel{N}{*}}$ & & เo & in & 0 & i̊. & $\wedge$ & $\begin{array}{l}\infty \\
0 \\
0\end{array}$ & $\stackrel{+}{+}$ & $\bar{\infty}_{\infty}$ & $\begin{array}{l}\infty \\
\infty\end{array}$ & స̃ & $\stackrel{\nabla}{\circ}$ & $\stackrel{\infty}{\sigma}$ & $\mp$ & $\stackrel{9}{\square}$ & 요 & 음 \\
\hline 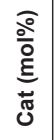 & ○े & ठ̊̊ & $\stackrel{\circ}{\circ}$ & $\stackrel{\circ}{\circ}$ & ঃ̊ & هั & هั & ठ̊ํ. & هั & @̊̀ & ঃ̊̀ & هั̊ & ठ̊̊ & $\begin{array}{l}\text { ̊̊̊ } \\
\stackrel{-}{-}\end{array}$ & $\stackrel{\circ}{\circ}$ & ه̊․ & $\stackrel{\text { ̊̊ }}{\stackrel{\circ}{\circ}}$ & $\stackrel{\text { ̊̊ }}{\dot{\circ}}$ & ஓ̊․ & 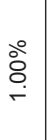 & ○ें & $\stackrel{\text { ̊̊̀ }}{\dot{-}}$ & ठ̊ํ் & $\stackrel{\text { ठ̊ }}{\circ}$ \\
\hline ల̃ & $\sum_{i=}^{\mathcal{M}}$ & $\sum_{i=}^{\mathcal{M}}$ & $\sum_{i=}^{\mathcal{p}}$ & $\sum_{i=}^{\mathcal{p}}$ & $\sum_{i=}^{\mathcal{D}}$ & $\sum_{i=}^{\vec{m}}$ & $\sum_{i=}^{\mathcal{M}}$ & $\sum_{i=}^{\mathcal{M}}$ & $\sum_{i=}^{\mathcal{M}}$ & $\sum_{i=}^{\stackrel{p}{m}}$ & $\sum_{i=}^{\vec{m}}$ & $\sum_{i=}^{\vec{m}}$ & $\sum_{i=}^{\not{m}}$ & $\sum_{i=}^{\vec{p}}$ & 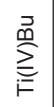 & $\sum_{i=}^{\vec{m}}$ & $\sum_{i=}^{\mathcal{M}}$ & 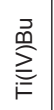 & $\sum_{i=}^{\stackrel{\vec{m}}{\underline{m}}}$ & $\sum_{i=}^{\stackrel{D}{M}}$ & 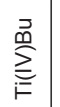 & 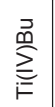 & $\sum_{i=}^{\stackrel{D}{\underline{m}}}$ & $\sum_{i=}^{\stackrel{p}{~}}$ \\
\hline 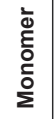 & 岀 & 岀 & 它 & 它 & 岀 & 岀 & 岀 & 它 & 岀 & 岀 & 它 & 它 & 岀 & 岀 & 岀 & 岀 & 它 & 产 & 它 & 它 & 战 & 它 & 它 & 它 \\
\hline
\end{tabular}


Table S1 - 4. Details of polymerization of 1,3-cyclopentanediol (CP) with either terephthalic acid (T), adipic acid(A) or sebacic acid (S) in the small-scale reactor. Catalyst loading is $1 \mathrm{~mol} \%$. Weight is in milligrams.

\begin{tabular}{|c|c|c|c|c|c|c|c|c|c|c|c|c|c|c|c|c|c|c|c|c|c|c|c|c|c|c|c|}
\hline$\underset{⺊}{\stackrel{O}{0}}$ & & & $\stackrel{\mathscr{m}}{\sim}$ & & & $\stackrel{\text { L }}{\sim}$ & & & $\stackrel{\dot{\leftrightarrow}}{\dot{E}}$ & & & 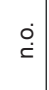 & & & $\begin{array}{l}\dot{\leftrightarrow} \\
\dot{C}\end{array}$ & & & $\begin{array}{l}\dot{\dot{C}} \\
\dot{\boldsymbol{C}}\end{array}$ & & & $\stackrel{\dot{\leftrightarrow}}{\dot{\Phi}}$ & & & $\begin{array}{l}\stackrel{N}{*} \\
\dot{J}\end{array}$ & & & 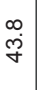 \\
\hline$\underset{0}{0}$ & & & $\begin{array}{l}\stackrel{\circ}{\dot{C}} \\
\stackrel{2}{2}\end{array}$ & & & $\begin{array}{l}\dot{\dot{E}} \\
\dot{I}\end{array}$ & & & $\begin{array}{l}\dot{\dot{\varphi}} \\
\dot{\Sigma}\end{array}$ & & & $\stackrel{\dot{\leftrightarrow}}{\dot{\Sigma}}$ & & & 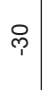 & & & 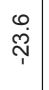 & & & $\begin{array}{l}\dot{\dot{C}} \\
\dot{\Sigma}\end{array}$ & & & $\begin{array}{l}\stackrel{9}{P} \\
\stackrel{p}{p}\end{array}$ & & & 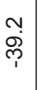 \\
\hline ه & 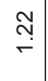 & $\stackrel{\underset{N}{\sim}}{ }$ & & $\hat{\stackrel{N}{i}}$ & 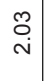 & & 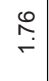 & $\stackrel{R}{\stackrel{R}{r}}$ & & $\stackrel{\substack{m \\
\sim}}{.}$ & $\stackrel{\leftrightarrow}{\leftarrow}$ & & $\underset{\sim}{\stackrel{N}{\sim}}$ & $\stackrel{\vec{m}}{\oplus^{\prime}}$ & & $\stackrel{\bar{n}}{\mathrm{i}}$ & $\stackrel{\leftrightarrow}{\mathrm{N}}$ & & 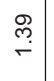 & 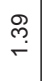 & & $\hat{m}$ & $\hat{m}$ & & $\stackrel{?}{\stackrel{N}{N}}$ & $\stackrel{?}{\sim}$ & \\
\hline 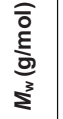 & 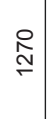 & \&্ণ & & $\begin{array}{l}\stackrel{0}{\infty} \\
\stackrel{0}{\circ}\end{array}$ & $\begin{array}{l}\stackrel{0}{0} \\
\stackrel{0}{\circ} \\
\stackrel{0}{\circ}\end{array}$ & & $\begin{array}{l}0 \\
\hat{0} \\
0\end{array}$ & 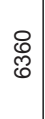 & & 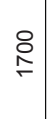 & 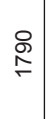 & & 胥 & $\begin{array}{l}\infty \\
\infty \\
\infty \\
\infty \\
\circ\end{array}$ & & 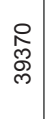 & $\begin{array}{l}\stackrel{0}{0} \\
b \\
b \\
b\end{array}$ & & 응 & 导 & & ঙ্ত & $\begin{array}{l}9 \\
\frac{1}{6} \\
6\end{array}$ & & 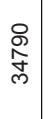 & $\begin{array}{l}8 \\
o \\
0 \\
0 \\
m\end{array}$ & \\
\hline $\begin{array}{l}\overline{\overline{0}} \\
\text { 을 } \\
\text { s. }\end{array}$ & O্ণ & ஜ్̊ㅇ & & 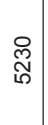 & 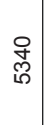 & & $\begin{array}{l}\text { : } \\
\text { ల్ }\end{array}$ & $\begin{array}{l}\text { 员 } \\
\text { 咞 }\end{array}$ & & $\underset{\stackrel{\text { I }}{\sim}}{ }$ & $\stackrel{\circ}{\stackrel{\circ}{\sim}}$ & & $\underset{\infty}{\infty}$ & $\begin{array}{l}\stackrel{P}{p} \\
\stackrel{2}{\circ}\end{array}$ & & $\begin{array}{l}\text { R } \\
0 \\
\stackrel{6}{\leftarrow}\end{array}$ & $\begin{array}{l}0 \\
\stackrel{1}{5} \\
\stackrel{5}{\leftarrow}\end{array}$ & & 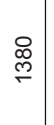 & 官 & & $\stackrel{R}{\stackrel{R}{i n}}$ & 을 & & 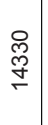 & 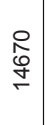 & \\
\hline 흥 & $\begin{array}{l}\frac{0}{\vec{z}} \\
\bar{\sigma} \\
\stackrel{0}{0}\end{array}$ & $\begin{array}{l}\frac{0}{3} \\
\bar{\sigma} \\
\frac{\pi}{0}\end{array}$ & $\begin{array}{l}\stackrel{\infty}{\vec{z}} \\
\stackrel{\mathrm{\sigma}}{0} \\
\stackrel{0}{0}\end{array}$ & $\stackrel{\frac{0}{5}}{\frac{5}{3}}$ & $\stackrel{\frac{0}{5}}{\frac{2}{3}}$ & $\stackrel{\frac{9}{5}}{\frac{2}{3}}$ & 畩 & $\stackrel{\frac{0}{5}}{\frac{2}{3}}$ & $\stackrel{\frac{0}{5}}{\frac{2}{3}}$ & 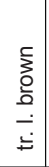 & 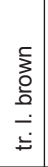 & 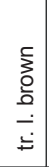 & 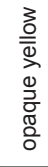 & 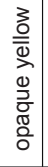 & 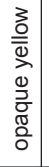 & 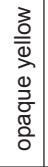 & 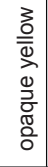 & $\begin{array}{l}3 \\
\frac{3}{\overline{0}} \\
\stackrel{0}{0} \\
\frac{\partial}{\bar{\sigma}} \\
\frac{\sigma}{0} \\
\end{array}$ & $\begin{array}{l}\frac{0}{3} \\
\frac{\sigma}{\sigma} \\
\frac{0}{0}\end{array}$ & $\begin{array}{l}\frac{1}{2} \\
\frac{\sigma}{\sigma} \\
\frac{0}{0}\end{array}$ & $\begin{array}{l}\frac{0}{2} \\
\frac{\sigma}{\sigma} \\
\frac{0}{0}\end{array}$ & $\stackrel{\frac{9}{5}}{\frac{5}{3}}$ & 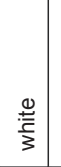 & 隹 & $\frac{0}{\frac{0}{3}}$ & $\stackrel{\frac{0}{5}}{\frac{\stackrel{5}{3}}{3}}$ & $\stackrel{0}{\frac{0}{3}}$ \\
\hline 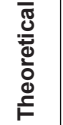 & $\frac{\circ}{\dot{m}}$ & $\stackrel{\circ}{\grave{m}}$ & $\frac{\circ}{m}$ & $\frac{\circ}{m}$ & $\frac{\circ}{\dot{m}}$ & $\frac{\circ}{\grave{m}}$ & $\frac{\circ}{m}$ & $\frac{\circ}{\grave{m}}$ & $\frac{\circ}{\bar{m}}$ & ঐें & ळे & ঐें & ळे & ळें & ๙ें & ळे & 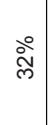 & ळे & $\stackrel{\circ}{\stackrel{\circ}{~}}$ & $\stackrel{\circ}{\stackrel{\circ}{\sim}}$ & $\stackrel{\circ}{\stackrel{\circ}{\sim}}$ & $\stackrel{\circ}{\stackrel{0}{0}}$ & 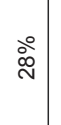 & 今े & œे & $\stackrel{\circ}{\stackrel{\circ}{~}}$ & 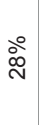 \\
\hline 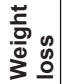 & $\frac{\circ}{6}$ & సิ่ & 今ें & $\stackrel{\text { ڤे }}{\circ}$ & $\stackrel{\circ}{\circ}$ & ڤे̀ & $\stackrel{\circ}{\stackrel{\circ}{\sim}}$ & $\stackrel{\circ}{\stackrel{0}{N}}$ & $\stackrel{\stackrel{\circ}{\sim}}{\mathrm{O}}$ & ঐे & 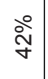 & $\stackrel{\text { ণे }}{\mathrm{\gamma}}$ & ڤें & ळें & ळें & $\stackrel{\circ}{\stackrel{\circ}{\circ}}$ & $\stackrel{\circ}{\grave{N}}$ & $\stackrel{\circ}{\stackrel{\circ}{\circ}}$ & 字 & $\stackrel{\circ}{\stackrel{\circ}{\circ}}$ & 号 & $\stackrel{\circ}{\circ}$ & ஃे & $\stackrel{\circ}{\stackrel{2}{\curvearrowright}}$ & ळें & $\stackrel{\circ}{\grave{\sim}}$ & ڤે̀ \\
\hline 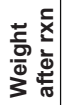 & $\stackrel{\leftrightarrow}{\dot{m}}$ & $\stackrel{\sim}{+}$ & เ & $\stackrel{\infty}{\sim}$ & $\begin{array}{l}\sigma \\
\infty \\
\infty\end{array}$ & $\stackrel{\varphi}{\Gamma}$ & $\stackrel{+}{\stackrel{\sim}{*}}$ & $\wedge$ & $\stackrel{\varphi}{\Gamma}$ & $\begin{array}{l}\infty \\
0 \\
0\end{array}$ & $\bar{\theta}$ & $\begin{array}{c}N \\
0\end{array}$ & $\underset{\infty}{m}$ & $\begin{array}{l}\infty \\
\infty \\
\infty\end{array}$ & $\stackrel{\overbrace{}}{\sim}$ & $\stackrel{\leftrightarrow}{\stackrel{\varphi}{\sim}}$ & $\stackrel{m}{\sim}$ & $\check{\Gamma}$ & $\stackrel{\overrightarrow{0}}{0}$ & $\begin{array}{l}\llcorner \\
\llcorner \\
\llcorner\end{array}$ & $\hat{\circ}$ & Na & $\begin{array}{l}0 \\
\infty\end{array}$ & $\underset{\infty}{N}$ & $\infty$ & $\begin{array}{c}\mathcal{N} \\
\infty\end{array}$ & $\stackrel{\infty}{\sim}$ \\
\hline 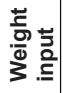 & N̦ & $\stackrel{\infty}{\circ}$ & $\circ$ & $\hat{\sigma}$ & $\begin{array}{l}\stackrel{\circ}{\circ} \\
\stackrel{\circ}{\circ}\end{array}$ & $\begin{array}{l}\omega \\
\infty \\
\infty\end{array}$ & $\stackrel{\infty}{\infty}$ & $\stackrel{\circ}{\circ}$ & 우 & $\stackrel{\infty}{\stackrel{\Gamma}{\leftarrow}}$ & $\begin{array}{l}0 \\
\stackrel{\circ}{\circ}\end{array}$ & $\begin{array}{l}\infty \\
\stackrel{\infty}{\circ}\end{array}$ & $\stackrel{\text { t. }}{\circ}$ & $\mp$ & $\begin{array}{l}\text { No } \\
\stackrel{\circ}{\circ}\end{array}$ & $\stackrel{?}{\stackrel{\rho}{\circ}}$ & $\circ$ & $\stackrel{\leftrightarrow}{\leftrightarrow}$ & $\stackrel{\stackrel{\leftrightarrow}{\rightleftarrows}}{\stackrel{\Gamma}{F}}$ & $\begin{array}{l}\text { 足 } \\
\stackrel{\circ}{\circ}\end{array}$ & $\begin{array}{l}\ddot{0} \\
\stackrel{\circ}{\circ}\end{array}$ & $\begin{array}{l}\infty \\
\stackrel{0}{\circ}\end{array}$ & $\begin{array}{l}\stackrel{0}{\circ} \\
\stackrel{\circ}{\circ}\end{array}$ & $\stackrel{\leftrightarrow}{\circ}$ & $\begin{array}{l}\stackrel{\leftrightarrow}{\circ} \\
\stackrel{\circ}{*}\end{array}$ & $\stackrel{\ominus}{\circ}$ & 우 \\
\hline $\overrightarrow{\tilde{J}}$ & ' & 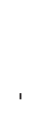 & . & $\sum_{i=}^{\stackrel{p}{m}}$ & 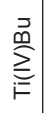 & $\sum_{i=}^{\mathfrak{m}}$ & 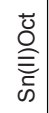 & 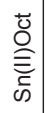 & 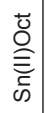 & & & . & 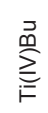 & 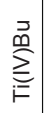 & 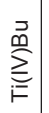 & 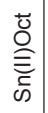 & 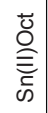 & $\begin{array}{l}\overrightarrow{\mathrm{o}} \\
\stackrel{0}{\hat{ٍ}} \\
\stackrel{c}{\omega}\end{array}$ & & . & , & 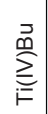 & 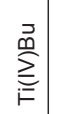 & $\sum_{i=}^{\stackrel{p}{m}}$ & 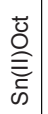 & 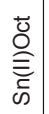 & 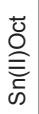 \\
\hline 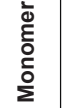 & $\begin{array}{l}t^{\prime} \\
\frac{d^{\prime}}{0}\end{array}$ & $\begin{array}{l}5 \\
\text { í }\end{array}$ & $\begin{array}{l}5 \\
0^{\prime}\end{array}$ & $\begin{array}{l}5 \\
\frac{1}{0}\end{array}$ & $\begin{array}{l}5 \\
\dot{1} \\
0\end{array}$ & $\begin{array}{l}5 \\
\dot{1}_{0}\end{array}$ & $\begin{array}{l}-5 \\
0_{0}^{\prime}\end{array}$ & $\begin{array}{l}-⿱ 亠 \\
\vdots \\
0 \\
0\end{array}$ & 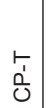 & $\begin{array}{l}\frac{\pi}{1} \\
\text { id }\end{array}$ & $\begin{array}{l}\frac{\pi}{1} \\
\text { in }\end{array}$ & $\begin{array}{l}\frac{\pi}{1} \\
\text { in }\end{array}$ & $\begin{array}{l}\frac{\pi}{1} \\
\text { i }\end{array}$ & $\begin{array}{l}\frac{\pi}{1} \\
\text { í }\end{array}$ & $\begin{array}{l}\frac{\pi}{1} \\
0 \\
0\end{array}$ & $\begin{array}{l}\frac{\pi}{1} \\
\text { ì }\end{array}$ & $\begin{array}{l}\frac{\pi}{1} \\
\text { in }\end{array}$ & $\begin{array}{l}\frac{\pi}{1} \\
\text { í }\end{array}$ & $\begin{array}{l}0 \\
1 \\
0 \\
0\end{array}$ & $\begin{array}{l}0 \\
1 \\
0 \\
0\end{array}$ & $\begin{array}{l}0 \\
1^{\prime} \\
0\end{array}$ & $\begin{array}{l}0 \\
0 \\
0\end{array}$ & $\begin{array}{l}0 \\
\dot{1} \\
0\end{array}$ & $\begin{array}{l}0 \\
1 \\
0 \\
0\end{array}$ & $\begin{array}{l}0 \\
1 \\
0 \\
0\end{array}$ & $\begin{array}{l}0 \\
1^{\prime} \\
0\end{array}$ & $\begin{array}{l}0 \\
1 \\
0 \\
0\end{array}$ \\
\hline
\end{tabular}


Table S1 - 5. Details of polymerization of 1,4-cyclohexanediol $(\mathrm{CH})$ with either terephthalic acid $(\mathrm{T})$, adipic acid(A) or sebacic acid (S) in the small-scale reactor. Catalyst loading is $1 \mathrm{~mol} \%$. Weight is in milligrams.

\begin{tabular}{|c|c|c|c|c|c|c|c|c|c|c|c|c|c|c|c|c|c|c|c|c|c|c|c|c|c|c|c|}
\hline 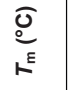 & & & $\begin{array}{l}\dot{\dot{\leftrightarrow}} \\
\dot{x}\end{array}$ & & & 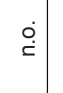 & & & $\begin{array}{l}\dot{\leftrightarrow} \\
\dot{\Sigma}\end{array}$ & & & $\begin{array}{l}\dot{\leftrightarrow} \\
\dot{c}\end{array}$ & & & $\begin{array}{l}\infty \\
\stackrel{\infty}{\oplus} \\
\stackrel{\leftarrow}{=}\end{array}$ & & & $\begin{array}{l}\stackrel{\rho}{\stackrel{\rho}{F}} \\
\stackrel{5}{\digamma}\end{array}$ & & & $\stackrel{n}{\stackrel{m}{m}}$ & & & 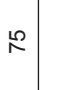 & & & $\frac{0}{\dot{\infty}}$ \\
\hline$\frac{0}{0}$ & & & $\stackrel{\dot{\leftrightarrow}}{\dot{\check{x}}}$ & & & $\stackrel{\dot{\varphi}}{\dot{I}}$ & & & $\stackrel{\dot{\leftrightarrow}}{\dot{\Sigma}}$ & & & $\stackrel{\stackrel{\leftrightarrow}{\dot{C}}}{ }$ & & & $\stackrel{\mathscr{\sigma}}{\dot{\sim}}$ & & & 10 & & & $\stackrel{\infty}{p}$ & & & $\underset{\Upsilon}{*}$ & & & 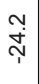 \\
\hline ه & $\stackrel{\infty}{\leftarrow}$ & $\stackrel{\infty}{\leftarrow}$ & & 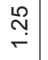 & $\stackrel{\overbrace{}}{\sim}$ & & 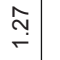 & 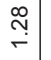 & & 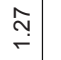 & 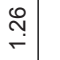 & & $\begin{array}{l}\stackrel{\leftrightarrow}{ } \\
\stackrel{N}{*}\end{array}$ & $\begin{array}{l}\mathscr{\infty} \\
\stackrel{\infty}{N}\end{array}$ & & 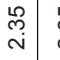 & 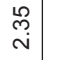 & & 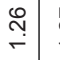 & 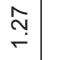 & & $\begin{array}{l}\hat{\oplus} \\
\dot{m}\end{array}$ & $\begin{array}{l}\hat{\omega} \\
\stackrel{N}{*}\end{array}$ & & $\overline{\mathrm{i}}$ & $\stackrel{\mathscr{\Omega}}{\leftarrow}$ & \\
\hline 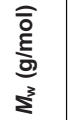 & $\stackrel{\stackrel{\leftrightarrow}{N}}{\sim}$ & $\stackrel{\stackrel{\leftrightarrow}{N}}{\sim}$ & & $\begin{array}{l}\stackrel{\circ}{\infty} \\
\stackrel{m}{\rightleftharpoons}\end{array}$ & $\begin{array}{l}\stackrel{\circ}{\circ} \\
\stackrel{m}{ }\end{array}$ & & 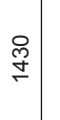 & 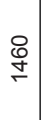 & & $\begin{array}{l}\stackrel{o}{o} \\
\stackrel{\sigma}{\sim}\end{array}$ & $\underset{g}{g}$ & & $\begin{array}{l}\text { : } \\
\text { ò } \\
\text { N. }\end{array}$ & $\begin{array}{l}\text { 导 } \\
\text { ồ } \\
\text { bे }\end{array}$ & & $\begin{array}{l}\stackrel{8}{\circ} \\
\stackrel{\infty}{-}\end{array}$ & 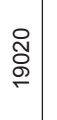 & & $\underset{g}{g}$ & 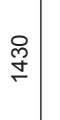 & & $\begin{array}{l}\stackrel{9}{9} \\
6 \\
o\end{array}$ & $\begin{array}{l}8 \\
8 \\
8 \\
\end{array}$ & & 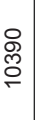 & $\begin{array}{l}\stackrel{\circ}{o} \\
\stackrel{\sigma}{\sigma}\end{array}$ & \\
\hline 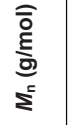 & $\stackrel{\circ}{\circ}$ & $\stackrel{0}{\circ}$ & & $\stackrel{\circ}{\circ}$ & $\stackrel{\circ}{\circ}$ & & $\stackrel{\stackrel{p}{m}}{=}$ & $\stackrel{g}{\stackrel{g}{\rightleftarrows}}$ & & $\stackrel{\text { 吊 }}{=}$ & 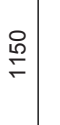 & & $\begin{array}{l}\text { Pै } \\
\text { पे } \\
\stackrel{0}{\circ}\end{array}$ & $\begin{array}{l}\stackrel{8}{\circ} \\
\stackrel{\circ}{\leftarrow}\end{array}$ & & 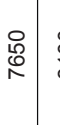 & $\frac{8}{\infty}$ & & $\stackrel{g}{\stackrel{g}{\rightleftarrows}}$ & $\stackrel{\stackrel{ }{ }}{\leftarrow}$ & & 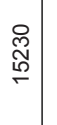 & $\begin{array}{l}\text { 융 } \\
\stackrel{0}{\leftarrow}\end{array}$ & & 弟 & \begin{tabular}{c}
0 \\
\multirow{1}{0}{} \\
1
\end{tabular} & \\
\hline$\frac{\text { 흥 }}{0}$ & 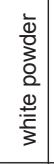 & 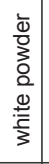 & 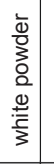 & 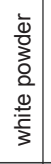 & 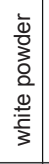 & 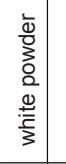 & 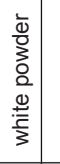 & 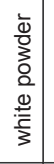 & 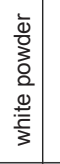 & 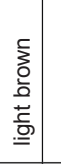 & 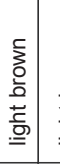 & 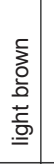 & 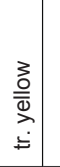 & 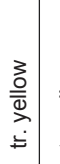 & $\begin{array}{l}\stackrel{3}{\partial} \\
\stackrel{\partial}{\bar{\omega}} \\
\stackrel{ \pm}{=}\end{array}$ & 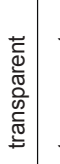 & 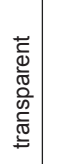 & 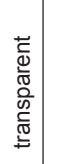 & $\begin{array}{l}\frac{1}{\vec{\sigma}} \\
\frac{\sigma}{\sigma} \\
\stackrel{0}{0}\end{array}$ & $\begin{array}{l}\frac{0}{3} \\
\frac{\sigma}{\sigma} \\
\stackrel{0}{0}\end{array}$ & $\begin{array}{l}\frac{0}{\vec{g}} \\
\frac{\mathrm{\sigma}}{\mathrm{\sigma}} \\
\stackrel{0}{0}\end{array}$ & 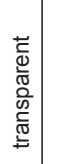 & 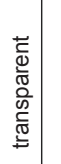 & 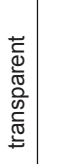 & $\begin{array}{l}0 \\
\frac{0}{\sigma} \\
\frac{\sigma}{0} \\
\stackrel{0}{0}\end{array}$ & 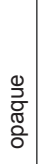 & $\begin{array}{l}\frac{0}{2} \\
\frac{\sigma}{\sigma} \\
\stackrel{0}{0}\end{array}$ \\
\hline 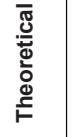 & ঐें & ๙্े & ঐें & ঐें & ๙े̀े & ঐे & ळें & ঐें & ঐें & ஓें & ஓें & ஓें & ১ें & ஓें & ১ें & ஓें & ஓें & $\stackrel{\circ}{\stackrel{े}{े}}$ & ১े & 今े & ळे & $\stackrel{\circ}{\stackrel{\circ}{2}}$ & 今े & ১ั & ळे & @̊ & ळ̊ \\
\hline 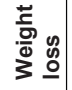 & ○े & ○̊ & ஓें & 今े & $\stackrel{\circ}{\circ}$ & $\stackrel{\circ}{\circ}$ & ○े & ஃ̊ & ঃ̊ & 官 & $\stackrel{\circ}{\stackrel{0}{*}}$ & $\stackrel{\circ}{\stackrel{\vartheta}{\Im}}$ & ঐे & 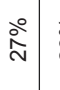 & ¿ें & ০ें & 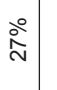 & 今े & ஓें & ○ें & $\stackrel{\circ}{\frac{\sigma}{\sigma}}$ & 今े & ণิ & $\stackrel{\circ}{\grave{N}}$ & ঃ̊ & ১ั & ڤે \\
\hline 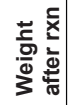 & 음 & $\begin{array}{l}\stackrel{N}{O} \\
\stackrel{0}{2}\end{array}$ & $\stackrel{\infty}{\infty}$ & $\hat{\sigma}$ & $\stackrel{m}{\circ}$ & $\stackrel{+}{\circ}$ & $\stackrel{\infty}{\infty}$ & $\begin{array}{l}\stackrel{0}{\circ} \\
\stackrel{\circ}{0}\end{array}$ & $\stackrel{\leftrightarrow}{\circ}$ & $\hat{\omega}$ & $\stackrel{\infty}{\infty}$ & 0 & $\stackrel{⿱ 亠}{\sim}$ & $\stackrel{?}{\sim}$ & $\stackrel{m}{\sim}$ & $\begin{array}{l}\stackrel{\infty}{0} \\
\dot{0}\end{array}$ & $\wedge$ & $\stackrel{\leftrightarrow}{\stackrel{0}{\sim}}$ & $\begin{array}{l}0 \\
\stackrel{0}{0}\end{array}$ & $\tilde{\omega}$ & $\overline{0}$ & $\stackrel{\leftrightarrow}{\sim}$ & $\check{\infty}_{\infty}^{-}$ & $\bar{\infty}_{\infty}$ & $\stackrel{\circ}{\wedge}$ & 市 & $\stackrel{\leftrightarrow}{\sim}$ \\
\hline 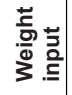 & $\div$ & \begin{tabular}{l}
$\stackrel{\sim}{\circ}$ \\
\hdashline
\end{tabular} & $\check{0}$ & $\hat{\sigma}$ & $\stackrel{\stackrel{\circ}{\circ}}{\sim}$ & $\begin{array}{l}\stackrel{0}{\circ} \\
\stackrel{0}{\circ}\end{array}$ & $\begin{array}{l}\infty \\
\stackrel{\infty}{\infty}\end{array}$ & $\begin{array}{l}\stackrel{0}{0} \\
\stackrel{0}{0}\end{array}$ & $\stackrel{\leftrightarrow}{\circ}$ & $\stackrel{+}{\circ}$ & $\begin{array}{l}m \\
\stackrel{0}{\circ}\end{array}$ & $\begin{array}{l}\stackrel{\leftrightarrow}{\circ} \\
\stackrel{\circ}{\circ}\end{array}$ & $\stackrel{10}{\circ}$ & $\stackrel{\circ}{\circ}$ & $\begin{array}{l}\infty \\
\sigma^{\infty}\end{array}$ & $\hat{\sigma}$ & $\begin{array}{l}\circ \\
\circ\end{array}$ & $\dot{\circ}$ & $\begin{array}{l}\infty \\
\infty \\
\infty\end{array}$ & $\stackrel{?}{\stackrel{0}{\circ}}$ & $\stackrel{+}{\circ}$ & $\begin{array}{l}\tilde{丶} \\
\stackrel{0}{\circ}\end{array}$ & $\begin{array}{l}0 \\
\stackrel{0}{\circ} \\
\stackrel{0}{2}\end{array}$ & $\begin{array}{l}\stackrel{N}{0} \\
\stackrel{0}{0}\end{array}$ & $\begin{array}{l}\stackrel{0}{\circ} \stackrel{0}{\circ} \\
\stackrel{2}{2}\end{array}$ & $\stackrel{\infty}{\infty}$ & $\stackrel{\circ}{\circ}$ \\
\hline తొ & . & ' & ' & $\sum_{i=}^{\stackrel{\Xi}{\underline{m}}}$ & 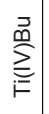 & 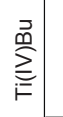 & 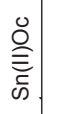 & 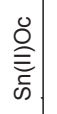 & 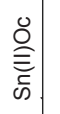 & , & , & . & $\sum_{i=\mid}^{\stackrel{m}{z}}$ & $\sum_{i=\mid}^{\underline{m}}$ & $\sum_{i=\mid}^{\stackrel{p}{\underline{m}}}$ & 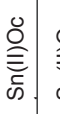 & 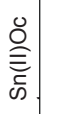 & 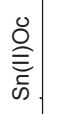 & 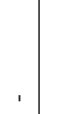 & , & , & $\sum_{i=}^{\stackrel{\Xi}{\underline{m}}}$ & $\sum_{i=}^{\stackrel{\vec{m}}{\underline{m}}}$ & $\sum_{i=}^{\underline{m}}$ & 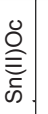 & 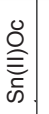 & 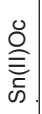 \\
\hline 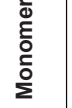 & $\begin{array}{l}\frac{1}{t} \\
\frac{1}{U}\end{array}$ & $\begin{array}{l}\frac{1}{\mathrm{I}} \\
\mathrm{I}\end{array}$ & $\begin{array}{l} \\
\grave{I} \\
\end{array}$ & 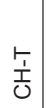 & $\begin{array}{l}\stackrel{\vdash}{\grave{I}} \\
\stackrel{0}{0}\end{array}$ & $\begin{array}{l}\frac{1}{T} \\
\frac{1}{U}\end{array}$ & $\begin{array}{l}\frac{5}{I} \\
\frac{1}{U}\end{array}$ & $\begin{array}{l}-⿱ 亠 䒑 \\
\frac{1}{U}\end{array}$ & $\frac{t_{1}}{\mathrm{I}}$ & $\begin{array}{l}\text { I⿱ } \\
\text { I⿱ }\end{array}$ & $\begin{array}{l}\mathbb{T} \\
\text { I⿱ } \\
U\end{array}$ & $\begin{array}{l}\frac{1}{\mathrm{I}} \\
\mathrm{U}\end{array}$ & $\begin{array}{l}\frac{1}{I} \\
\stackrel{I}{0}\end{array}$ & $\begin{array}{l}\frac{T}{I} \\
\stackrel{I}{U}\end{array}$ & $\begin{array}{l}\frac{1}{I} \\
\stackrel{I}{0}\end{array}$ & $\begin{array}{l}\mathbb{T} \\
\underline{I} \\
0\end{array}$ & $\begin{array}{l}\frac{1}{I} \\
\stackrel{I}{U}\end{array}$ & $\begin{array}{l}\frac{1}{I} \\
\stackrel{I}{0}\end{array}$ & $\begin{array}{l}0 \\
⿱ 亠 䒑 \\
\\
0\end{array}$ & $\begin{array}{l}\text { Op } \\
\text { I } \\
\end{array}$ & $\begin{array}{l}0 \\
\stackrel{1}{I} \\
\end{array}$ & $\begin{array}{l}0 \\
\stackrel{1}{I} \\
\text { U. }\end{array}$ & $\begin{array}{l}0 \\
\stackrel{1}{I} \\
\text { U. }\end{array}$ & $\begin{array}{l}0 \\
⿱ 亠 䒑 \\
\end{array}$ & $\begin{array}{l}0 \\
\frac{1}{J} \\
\end{array}$ & $\begin{array}{l}0 \\
⿱ 亠 䒑 \\
\\
\end{array}$ & $\begin{array}{l}0 \\
\frac{1}{I} \\
\end{array}$ \\
\hline
\end{tabular}


Table S1 - 6. Details of polymerization of 1,4-cyclohexanedimethanol ( $\mathrm{CHdm})$ with either terephthalic acid ( $\mathrm{T}$ ), adipic $\operatorname{acid}(A)$ or sebacic acid $(S)$ in the small-scale reactor. Catalyst loading is $1 \mathrm{~mol} \%$. Weight is in milligrams.

\begin{tabular}{|c|c|c|c|c|c|c|c|c|c|c|c|c|c|c|c|c|c|c|c|c|c|c|c|c|c|c|c|}
\hline$\underset{1}{0}$ & & & $\begin{array}{l}\stackrel{\sim}{+} \\
\stackrel{+}{\sim}\end{array}$ & & & $\stackrel{\dot{\leftrightarrow}}{\dot{\Sigma}}$ & & & $\stackrel{\circ}{\circ}$ & & & $\stackrel{\infty}{\dot{\infty}}$ & & & ळ & & & $\begin{array}{l}\text { Lొ } \\
\text { హె }\end{array}$ & & & $\hat{\dot{q}}$ & & & $\begin{array}{l}\stackrel{+}{o} \\
\dot{\alpha}\end{array}$ & & & $\check{\forall}$ \\
\hline 苟 & & & $\begin{array}{l}\dot{0} \\
\dot{\Sigma}\end{array}$ & & & $\begin{array}{l}\dot{\dot{x}} \\
\dot{\dot{x}}\end{array}$ & & & $\begin{array}{l}\dot{\dot{x}} \\
\dot{\Sigma}\end{array}$ & & & $\stackrel{\circ}{\dot{\leftrightarrow}}$ & & & $\stackrel{\dot{\leftrightarrow}}{\dot{C}}$ & & & $\stackrel{\dot{\rho}}{\dot{c}}$ & & & $\stackrel{\dot{\rho}}{\dot{c}}$ & & & $\begin{array}{l}\dot{\leftrightarrow} \\
\dot{x}\end{array}$ & & & 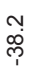 \\
\hline هـ & $\stackrel{\stackrel{f}{\leftarrow}}{\circ}$ & $\begin{array}{l}\mathscr{O} \\
\stackrel{\leftrightarrow}{-}\end{array}$ & & 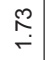 & $\stackrel{⿱}{\stackrel{5}{r}}$ & & 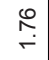 & $\stackrel{⿱}{\stackrel{丶}{r}}$ & & $\stackrel{\mathscr{R}}{\stackrel{\circ}{\sim}}$ & $\stackrel{n}{\sim}$ & & $\stackrel{\mathcal{N}}{\mathrm{N}}$ & $\stackrel{\mathbb{N}}{\mathrm{N}}$ & & $\stackrel{\sim}{N}$ & $\stackrel{\widetilde{N}}{\sim}$ & & $\stackrel{\hat{\leftrightarrow}}{\stackrel{-}{-}}$ & $\stackrel{\leftrightarrow}{\leftrightarrow}$ & & $\begin{array}{c}\stackrel{0}{\circ} \\
\stackrel{N}{N}\end{array}$ & $\underset{\stackrel{N}{\sim}}{\mathfrak{N}}$ & & $\stackrel{\mathscr{N}}{N}$ & $\stackrel{N}{\stackrel{N}{N}}$ & \\
\hline इ 흥 & 인 & $\begin{array}{l}\stackrel{8}{0} \\
\stackrel{\circ}{\leftarrow}\end{array}$ & & 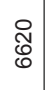 & 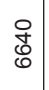 & & $\stackrel{\infty}{\infty}$ & $\begin{array}{l}\stackrel{\circ}{\circ} \\
\stackrel{\circ}{\wedge}\end{array}$ & & $\begin{array}{l}: \\
\stackrel{\leftrightarrow}{*} \\
N\end{array}$ & 怘 & & 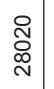 & $\underset{\substack{0 \\
\stackrel{2}{N}}}{\stackrel{0}{2}}$ & & 응 & $\begin{array}{l}\stackrel{\mathbb{N}}{ } \\
\text { N }\end{array}$ & & $\begin{array}{l}\circ \\
: \\
\stackrel{+}{+}\end{array}$ & $\frac{0}{\dot{q}}$ & & 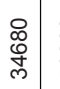 & 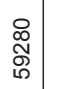 & & 응 & $\begin{array}{l}\stackrel{P}{+} \\
\stackrel{N}{N}\end{array}$ & \\
\hline $\begin{array}{l}\widehat{\overline{0}} \\
\text { g్ } \\
\text { s్ }\end{array}$ & $\begin{array}{l}0 \\
\stackrel{0}{0}\end{array}$ & 웅 & & $\stackrel{\circ}{\circ}$ & $\stackrel{\circ}{\circ}$ & & $\begin{array}{l}0 \\
o \\
o \\
q\end{array}$ & $\begin{array}{l}0 \\
\stackrel{0}{a}\end{array}$ & & 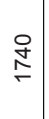 & 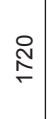 & & $\begin{array}{l}\stackrel{8}{0} \\
\stackrel{0}{ }\end{array}$ & 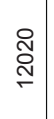 & & 旁 & $\begin{array}{l}\stackrel{8}{\circ} \\
\stackrel{\circ}{ }\end{array}$ & & 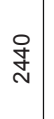 & $\stackrel{\text { ָ̊ }}{\mathrm{Y}}$ & & 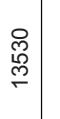 & 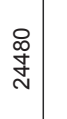 & & $\begin{array}{l}\stackrel{\circ}{\circ} \\
\stackrel{\circ}{\leftarrow}\end{array}$ & 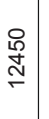 & \\
\hline 흥 & $\frac{0}{\frac{2}{3}}$ & 产 & $\stackrel{\frac{9}{3}}{\frac{3}{3}}$ & $\frac{\stackrel{2}{\frac{5}{3}}}{\frac{3}{3}}$ & $\frac{\stackrel{9}{\frac{5}{3}}}{\frac{1}{3}}$ & $\stackrel{\frac{2}{3}}{\frac{2}{3}}$ & $\frac{0}{\frac{\pi}{3}}$ & $\stackrel{\frac{2}{5}}{\frac{5}{3}}$ & $\frac{\stackrel{9}{\frac{5}{3}}}{\frac{3}{3}}$ & $\begin{array}{l}\frac{0}{\vec{g}} \\
\frac{\mathrm{\sigma}}{\mathrm{g}} \\
\frac{\mathrm{o}}{0}\end{array}$ & $\begin{array}{l}\frac{0}{\vec{z}} \\
\overline{\mathrm{J}} \\
\frac{\mathrm{o}}{0}\end{array}$ & $\begin{array}{l}\frac{0}{z} \\
\frac{\sigma}{\pi} \\
\frac{0}{0}\end{array}$ & 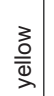 & 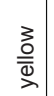 & $\begin{array}{l}3 \\
\stackrel{3}{\bar{D}} \\
\end{array}$ & $\begin{array}{l}\frac{0}{g} \\
\bar{\sigma} \\
\frac{\sigma}{0} \\
\end{array}$ & $\begin{array}{l}\frac{0}{J} \\
\frac{\sigma}{\sigma} \\
\frac{0}{0}\end{array}$ & $\begin{array}{l}\frac{0}{\vec{g}} \\
\frac{\pi}{0} \\
\frac{\mathrm{o}}{0}\end{array}$ & $\begin{array}{l}\frac{0}{3} \\
\bar{\sigma} \\
\frac{\pi}{0} \\
0\end{array}$ & $\begin{array}{l}\frac{0}{\vec{z}} \\
\overline{\mathrm{\sigma}} \\
\frac{\mathrm{O}}{0}\end{array}$ & $\begin{array}{l}\stackrel{0}{\vec{z}} \\
\frac{\mathrm{\sigma}}{0} \\
\frac{0}{0}\end{array}$ & $\begin{array}{l}\stackrel{0}{\vec{z}} \\
\frac{\mathrm{g}}{\mathrm{\sigma}} \\
\frac{0}{0}\end{array}$ & 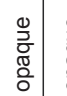 & $\begin{array}{l}\frac{0}{2} \\
\frac{\vec{\sigma}}{0} \\
\frac{0}{0}\end{array}$ & $\begin{array}{l}\frac{0}{2} \\
\frac{\vec{\sigma}}{0} \\
\frac{0}{0}\end{array}$ & $\begin{array}{l}\frac{\Phi}{2} \\
\frac{\bar{\sigma}}{0} \\
\frac{0}{0}\end{array}$ & $\begin{array}{l}\frac{0}{2} \\
\frac{7}{0} \\
\frac{0}{0}\end{array}$ \\
\hline 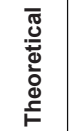 & $\stackrel{\circ}{\stackrel{े}{े}}$ & 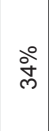 & 啇 & ஓें & $\stackrel{\circ}{\stackrel{े}{े}}$ & 广े & $\stackrel{\circ}{\grave{j}}$ & @े & 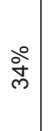 & ¿ें & 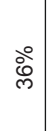 & 吕 & 仓ें & ळ & ¿े & \&े & 仓े & @े & ڤ్లે & ڤે & ڤ్లે & స్లి & సें & ڤેं & ঐे & స్లે & స్లે \\
\hline 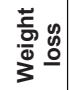 & 今ั & ○े & $\stackrel{\circ}{\circ}$ & $\stackrel{\circ}{\circ}$ & $\stackrel{\circ}{\stackrel{\circ}{\circ}}$ & $\stackrel{\circ}{\stackrel{\circ}{\circ}}$ & ઠें & ஓे & $\stackrel{\circ}{\stackrel{2}{人}}$ & 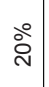 & $\stackrel{\circ}{\grave{N}}$ & ஓे & ळें & $\stackrel{\circ}{\stackrel{\circ}{~}}$ & ลे & $\stackrel{\circ}{\grave{N}}$ & $\stackrel{\circ}{\grave{N}}$ & $\stackrel{\circ}{\stackrel{N}{N}}$ & $\stackrel{\circ}{\circ}$ & 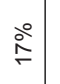 & ㅇํ & $\stackrel{\circ}{\stackrel{\circ}{N}}$ & $\stackrel{\circ}{\circ}$ & $\stackrel{\circ}{\stackrel{\circ}{~}}$ & 움 & $\stackrel{\circ}{\circ}$ & $\stackrel{\circ}{\stackrel{\circ}{\circ}}$ \\
\hline 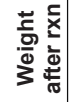 & $\dot{\sigma}$ & 0 & $\stackrel{10}{\infty}$ & $\infty$ & $\stackrel{+}{\infty}$ & $\stackrel{\leftrightarrow}{\wedge}$ & $\begin{array}{c}m \\
\infty \\
\infty\end{array}$ & $\stackrel{+}{\infty}$ & $\begin{array}{c}m \\
\infty\end{array}$ & $\stackrel{⿱ 亠 乂}{\sim}$ & 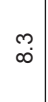 & $\begin{array}{c}m \\
\infty \\
\infty\end{array}$ & $\stackrel{\imath}{\wedge}$ & $\stackrel{\circ}{\stackrel{\circ}{\sim}}$ & $\stackrel{\varphi}{\sim}$ & $\stackrel{m}{\infty}$ & $\stackrel{\text { カ }}{\sim}$ & $\bar{\infty}_{\infty}^{-}$ & $\widehat{\sim}$ & $\begin{array}{l}0 \\
\infty\end{array}$ & $\infty$ & $\stackrel{\infty}{\circ}$ & $\stackrel{\text { L }}{\sim}$ & ז. & $\stackrel{\widehat{\infty}}{\infty}$ & $\underset{\infty}{N}$ & $\infty$ \\
\hline 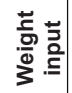 & 으 & 으 & $\begin{array}{l}0 \\
\stackrel{0}{0}\end{array}$ & $\stackrel{\infty}{\infty}$ & $\stackrel{N}{\circ}$ & $\stackrel{\circ}{\circ}$ & $\begin{array}{l}\stackrel{t}{0} \\
\stackrel{0}{2}\end{array}$ & $\stackrel{+}{\circ}$ & 으 & N̦ & $\begin{array}{l}\llcorner \\
\stackrel{0}{\circ}\end{array}$ & ֻ̃ & 음 & 우 & $\stackrel{\infty}{\infty}$ & $\begin{array}{l}\stackrel{0}{\circ} \\
\stackrel{0}{\circ}\end{array}$ & $\stackrel{\leftrightarrow}{\circ}$ & $\stackrel{\sim}{\stackrel{0}{0}}$ & @̊m.ㅇ & $\begin{array}{l}m \\
\stackrel{0}{\circ}\end{array}$ & $\stackrel{\leftrightarrow}{\circ}$ & $\stackrel{m}{\infty}$ & $\stackrel{\sim}{\stackrel{0}{\circ}}$ & $\stackrel{\infty}{\infty}$ & ำ & $\stackrel{\infty}{\sigma}$ & $\hat{\sigma}$ \\
\hline ల్ & . & . & , & 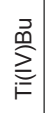 & 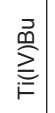 & 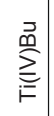 & 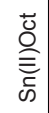 & 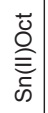 & 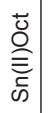 & ' & & & 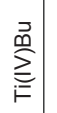 & $\sum_{i=}^{\stackrel{g}{m}}$ & 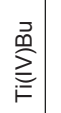 & 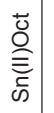 & 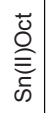 & 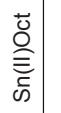 & & & & $\sum_{i=}^{\stackrel{p}{\underline{m}}}$ & 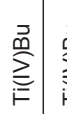 & $\sum_{i=}^{\mathfrak{m}}$ & 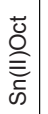 & 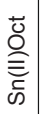 & 岕 \\
\hline 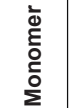 & $\begin{array}{l}\vdash \\
\frac{1}{\dot{\varepsilon}} \\
\frac{0}{\tau} \\
\frac{1}{U}\end{array}$ & $\mid \begin{array}{l}- \\
\frac{1}{\varepsilon} \\
\frac{0}{I} \\
\text { U. }\end{array}$ & 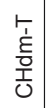 & 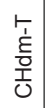 & 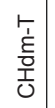 & 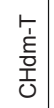 & 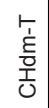 & 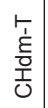 & 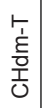 & 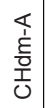 & $\begin{array}{l}\mathbb{T} \\
\dot{\varepsilon} \\
\frac{0}{1} \\
0\end{array}$ & 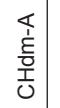 & 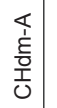 & 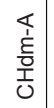 & $\begin{array}{l}\mathbb{T} \\
\dot{\xi} \\
\text { 吾 } \\
0\end{array}$ & 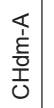 & 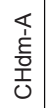 & $\begin{array}{l}\text { 조 } \\
\text { 훙 } \\
\text { 동 }\end{array}$ & 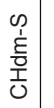 & 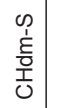 & 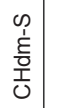 & 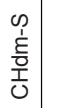 & 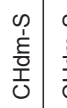 & 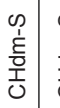 & 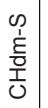 & 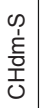 & 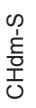 \\
\hline
\end{tabular}




\section{Chapter 2 The curious case of 1,3-cyclopentanediol}

This chapter is based on the publication:

Small-scale screening of novel biobased monomers: the curious case of 1,3-cyclopentanediol.

G.J. Noordzija,b, C.H.J.T. Dietz ${ }^{c, b}$, N. Leonéa , C.H.R.M. Wilsens ${ }^{a}$, S. Rastogia

RSC Advances, 2018, 8, 39818

DOI: $10.1039 / c 8 r a 08811 \mathrm{j}$

a) Aachen-Maastricht Institute of Biobased Materials (AMIBM), Faculty of Science and Engineering, Maastricht University, Brightlands Chemelot Campus, 6167 RD Geleen, The Netherlands.

b) Chemelot InSciTe, Urmonderbaan 20F, NL-6167 RD Geleen, The Netherlands.

c)Inorganic Membranes and Membrane Reactors, Dept. Chemical Engineering and Chemistry, Eindhoven University of Technology, PO Box 513, Eindhoven, The Netherlands.

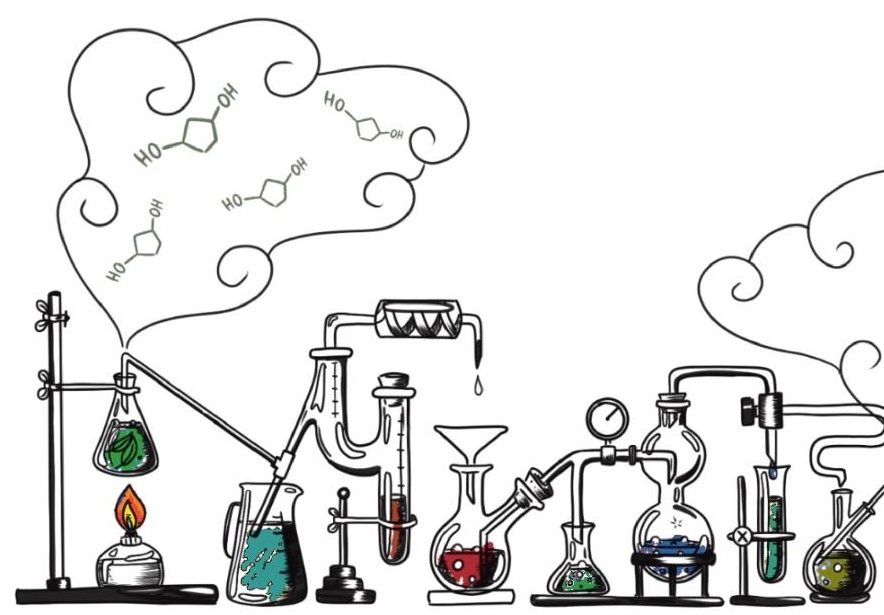




\section{Abstract}

In this work, we report on the small scale polycondensation and consecutive analysis of novel polyesters based on the potentially renewable 1,3-cyclopentanediol (CPdiol). To avoid evaporation of monomers during thin-film polymerization reactions, trimer pre-polyesters have been synthesized from the corresponding acid-chlorides with diol monomers. Polymerization of these trimers was explored in thermogravimetric analysis to identify potential side reactions, and to assess the ideal polymerization temperature. In general we observe that trans-1,3cyclopentanediol exhibits good thermal stability up to $200{ }^{\circ} \mathrm{C}$, whereas thermal dehydration of the alcohol end-groups occurs upon further heating. In contrast, for cis-1,3-cyclopentanediol, the ester bonds of the cyclopentane end-groups become labile, thereby generating carboxylic acid end-groups, and 3-cyclopentenol already at $180{ }^{\circ} \mathrm{C}$. The thermal dehydration reactions yield double bond end-groups, which in turn facilitate cross-linking through cross-coupling and Diels-Alder reactions, leading to an increase in molecular weight. Despite the limited thermal stability of CPdiol, here we demonstrate that polymerization of CPdiol can successfully be achieved in thin-film polycondensation conditions at $180{ }^{\circ} \mathrm{C}$, yielding molecular weights well above $10 \mathrm{~kg} / \mathrm{mol}$.

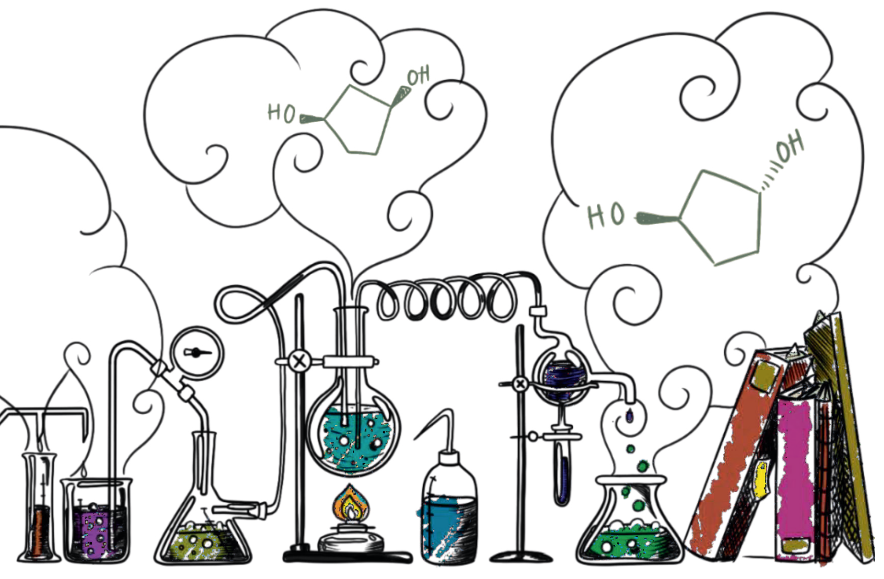




\section{Introduction}

The first chapter in this thesis describes the small-scale polycondensation of 1,3-cyclopentanediol (CPdiol), a potentially renewable monomer obtainable from furfuryl alcohol. ${ }^{1}$ Subsequent comparison of polyesters with CPdiol as building block was compared to commercially relevant cyclic diols 1,4-cyclohexanediol (CHdiol) and 1,4-cyclohexanedimethanol $(\mathrm{CHdmol})$. There it was found that in terms of thermal properties polyesters of CPdiol behaved similarly to polyesters with $\mathrm{CHdmol}$, as similar glass-transition temperatures $\left(T_{\mathrm{g}}\right)$ and melting temperatures $\left(T_{\mathrm{m}}\right)$ were found, with the exception of poly(CPdiol-adipate) which did not exhibit crystallization and melting behavior. At the same time it was found that polymers of CPdiol with terephthalate $(T)$ and 2,5-furandicarboxylate (FDCA) yielded semi-crystalline polyesters with a $T_{\mathrm{m}}$ well above $240{ }^{\circ} \mathrm{C}$. However, these polyesters also exhibit an unstable melt, as is suggested by strong discoloration of the polymer upon melting.

Similar to other cyclic diols, 1,3-CPdiol is obtained as a racemic mixture, and typically in racemic cycloaliphatic monomers the trans isomer results in higher rigidity in the polymer, resulting in an increase in glass transition temperature $\left(T_{\mathrm{g}}\right)$. Higher symmetry of the trans isomer also results into more perfect crystals, favoring higher crystallinity, as opposed to the cis isomer. This effect is well described for polyesters having cycloaliphatic rings, for example $\mathrm{CHdiol}^{2,3}, \mathrm{CHdmol}^{4}$, and others. ${ }^{3,5-8}$ In most cases the polyesters with a high trans ratio are semi-crystalline, whereas crystallinity is often lost when reaching, or exceeding, a 50/50 cis/trans mixture. With this literature precedent it is expected that the properties of polymers with CPdiol will vary with the cis/trans ratio. Additionally, compared to six-membered rings, the five membered cycloaliphatic ring in CPdiol is expected to suppress rigidity and symmetry. ${ }^{9}$ This is due to the steric configuration of the cyclopentane ring, which cannot adopt the well-known 'boat'-conformation found in cyclohexane. ${ }^{10}$

To study the effect on thermal properties of polymers with CPdiol, the cis/trans ratio was varied by fraction distillation under vacuum. Then, following the synthesis protocol described in chapter 1, trimer pre-polyesters were prepared with FDCA, followed by small-scale screening polymerization to assess the effect of the cis/trans ratio on the polymer properties. 


\section{Experimental section}

\section{Materials}

1,3-cyclopentanediol (15/85 cis/trans) and anhydrous chloroform were obtained from Sigma Aldrich. Magnesium (IV) sulfate, 4-dimethylaminopyridine and pyridine were obtained from ACROS. 2,5-furandicarboxylic acid was obtained from ABCR. Standard laboratory solvents were obtained from Biosolve. Deuterated solvents were obtained from Buchem BV (Netherlands). The purchased compounds were used directly without further purification, unless otherwise specified.

\section{Characterization methods}

${ }^{1} \mathrm{H}-\mathrm{NMR}$ and ${ }^{13} \mathrm{C}-\mathrm{NMR}$ spectra were recorded with a Bruker Ultrashield 300 spectrometer $(300 \mathrm{MHz}$ magnetic field). NMR-samples were prepared by dissolving ca. $10 \mathrm{mg}$ of sample in $0.5 \mathrm{~mL}$ deuterated solvent, including dimethyl sulfoxide (DMSO-d $\left.d_{6}\right)$, deuterated chloroform $\left(\mathrm{CDCl}_{3}\right)$, and deuterated trifluoroacetic acid ( $d$-TFA). All spectra were referenced against tetramethylsilane (TMS), or residual solvent peak from the deuterated solvent. Molecular weight $\left(M_{n}, M_{w}\right)$ and dispersity $(\Xi)$ of the polymers were calculated after gel permeation chromatography (GPC) on a PSS SECcurity GPC system using Agilent 1260 Infinity instrument technology. The GPC system was equipped with two PFG combination medium micro-columns with $7 \mu \mathrm{m}$ particle size $(4.6 \times 250 \mathrm{~mm}$, separation range 100-1.000.000 Da), a PFG combination medium pre-column with $7 \mu \mathrm{m}$ particle size $(4.6 \times 30 \mathrm{~mm})$, and a Refractive Index detector (RI). Distilled 1,1,1,3,3,3-hexafluoroisopropanol (HFIP) containing $0.019 \%$ sodium trifluoroacetate was used as mobile phase at $40{ }^{\circ} \mathrm{C}$, with a $0.3 \mathrm{~mL} / \mathrm{min}$ flow rate. The GPC apparatus was calibrated with poly(methyl methacrylate) standards obtained from PSS. GPC samples were prepared by dissolving $5 \mathrm{mg}$ of polymer in $1.5 \mathrm{~mL} \mathrm{HFIP}$ overnight under constant shaking, the samples were filtered over a $0.2 \mu \mathrm{m}$ PTFE syringe filter prior to injection. Thermal stability of compounds and screening of polymerization conditions were performed via thermogravimetric analysis (TGA) using a TA Instruments Q500. Experiments were performed under a nitrogen atmosphere with a heating rate of $10{ }^{\circ} \mathrm{C} / \mathrm{min}$, from room temperature (RT) up to $700{ }^{\circ} \mathrm{C}$. For polymerization screening experiments the sample was heated to the desired reaction temperature (generally 180,200 , or $220^{\circ} \mathrm{C}$ ) at a rate of $10^{\circ} \mathrm{C} / \mathrm{min}$ and kept isothermal for the desired reaction time. Thermal transition temperatures of the polymers were analyzed via differential scanning calorimetry (DSC) using a TA Instruments DSC Q2000. Typically, two heating and cooling runs were performed at $10^{\circ} \mathrm{C} / \mathrm{min}$, where the first heating was used to erase any thermal history in the samples. The glass-transition $\left(T_{\mathrm{g}}\right)$, melt-transition $\left(T_{\mathrm{m}}\right)$, crystallization $\left(T_{\mathrm{c}}\right)$, and cold-crystallization $\left(T_{\mathrm{cc}}\right)$ temperatures were obtained from the second heating and cooling run. DSC samples were prepared by loading $3-5 \mathrm{mg}$ oven-dried samples in Tzero Hermetic Aluminium pans. Polarized optical microscopy (POM) images were recorded on an Olympus BX53DP 26, equipped with a Linkam HFSX350 Hotstage. POM was used to determine melt-temperatures of samples which could not be measured via DSC (e.g. because of degradation upon melting). POM samples were loaded on a microscopy slide and heated at a rate of 10 ${ }^{\circ} \mathrm{C} / \mathrm{min}$. The melt-temperature or range was determined by visual aid of the samples becoming isotropic in nature. Matrix assisted laser desorption/ionization-time of flight-mass spectroscopy (MALDI-ToF-MS) analysis was recorded on a Voyager DESTR from Applied Biosystems (laser frequency $20 \mathrm{~Hz}, 337 \mathrm{~nm}$ and a voltage of $25 \mathrm{kV})$. The matrix material was DCTB (40 mg/mL in HFIP). Potassium trifluoroacetate was added as cationic ionization agent $(5 \mathrm{mg} / \mathrm{mL}$ in HFIP). The polymer sample was dissolved in HFIP (1 $\mathrm{mg} / \mathrm{mL}$ ), to which the matrix material and the ionization agent were added (5:1:5). If the samples were not fully dissolved, the mixture was filtered over a $0.2 \mu \mathrm{m}$ PTFE syringe filter prior to placing on the target plate. 


\section{Synthesis methods}

\section{Enantiomeric enrichment of 1,3-cyclopentanediol via fractional distillation}

1,3-Cyclopentanediol from Sigma Aldrich was obtained as an cis/trans enantiomeric mixture having $>95 \%$ purity. The commercial batch was analyzed prior to use via NMR and chiral GC to determine the cis/trans ratio, and was found to be 15/85 cis/trans (see SI for analysis). Based on the different boiling point of the cis/trans enantiomers, the commercial batch was purified by fractional distillation under vacuum. Various cis/trans ratios were obtained via batch-wise fractional distillation; Generally, 1,3-cyclopentanediol was loaded in a $50 \mathrm{~mL} 2$-neck round-bottom flask, equipped with a $10 \mathrm{~cm}$ vigreux column, distillation 3-way adapter, condenser path, and a vacuum adapter to collect different fractions. Temperature of the oil-bath was varied from $130{ }^{\circ} \mathrm{C}$ to $160^{\circ} \mathrm{C}$. Temperature of the boiling phase was measured in the 3-way adapter, and the depth of the vacuum was noted. All fractions were obtained either as colorless liquids or as colorless solids: $7.6 \%$ cis $\left(110{ }^{\circ} \mathrm{C}, 0.85 \mathrm{mbar}\right), 14 \%$ cis $\left(125^{\circ} \mathrm{C}, 1.4 \mathrm{mbar}\right), 20.7 \%$ cis $\left(120^{\circ} \mathrm{C}, 1.6 \mathrm{mbar}\right)$, $30 \%$ cis $\left(116{ }^{\circ} \mathrm{C}, 1.2 \mathrm{mbar}\right), 41 \%$ cis $\left(111^{\circ} \mathrm{C}, 1.3 \mathrm{mbar}\right)$.

\section{General synthesis of trimer pre-polyesters}

The synthesis methods of trimer pre-polyesters were the same as described in chapter 1.

\section{Trimer 1,3-cyclopentanediol-furanoate, $-8 \%$ cis}

The product was obtained as white solids $\left(1.12 \mathrm{~g}, 67 \%\right.$ yield). After drying at $110^{\circ} \mathrm{C}$ discoloration occurred, the product was isolated by dissolving the mix in $\mathrm{CHCl}_{3}$, filter out the brown solids, and reduce the $\mathrm{CHCl}_{3}$ in vacuo, to re-obtain the product as white solids $\left(0.64 \mathrm{~g}, 38 \%\right.$ yield). ${ }^{1} \mathrm{H} \mathrm{NMR}\left(\mathrm{CDCl}_{3}, 300 \mathrm{MHz}\right): \delta 7.12$ $(\mathrm{m}, 2 \mathrm{H}), 5.46(\mathrm{~m}, 2.27 \mathrm{H}), 4.60(\mathrm{~m}, 1.74 \mathrm{H}), 4.51(\mathrm{~m}, 0.16 \mathrm{H}), 2.22(\mathrm{~m}, 2.53 \mathrm{H}), 2.12(\mathrm{~m}, 5.91 \mathrm{H}), 1.85(\mathrm{~m}$, 2.56H), $1.73(\mathrm{~m}, 1.82 \mathrm{H}) .{ }^{13} \mathrm{C} \mathrm{NMR}\left(\mathrm{CDCl}_{3}, 300 \mathrm{MHz}\right): \delta 158.4(\mathrm{C}=\mathrm{O}), 146.8(\mathrm{C}-\mathrm{O}$, furan ring) $118.7(\mathrm{C}-\mathrm{C}$, furan ring) 79.7 ( $\mathrm{CH}-\mathrm{OR}$ ring) $73.2(\mathrm{CH}-\mathrm{OH}$ ring $), 41.6\left(\mathrm{CH}_{2}\right.$ ring $), 32.8\left(\mathrm{CH}_{2}\right.$ ring $), 30.3\left(\mathrm{CH}_{2}\right.$ ring $)$.

\section{Trimer 1,3-cyclopentanediol-furanoate, $-20 \%$ cis}

The product was obtained as off-white solids $\left(1.34 \mathrm{~g}, 80 \%\right.$ yield). After drying at $110{ }^{\circ} \mathrm{C}$ discoloration occurred, the product was isolated by dissolving the mix in $\mathrm{CHCl}_{3}$, filter out the brown solids, and reduce the $\mathrm{CHCl}_{3}$ in vacuo, to re-obtain the product as off-white solids $(0.88 \mathrm{~g}, 52 \%$ yield $) .{ }^{1} \mathrm{H} \mathrm{NMR}\left(\mathrm{CDCl}_{3}, 300\right.$ $\mathrm{MHz})$ : $\delta 7.12(\mathrm{~m}, 2 \mathrm{H}), 5.46(\mathrm{~m}, 2.27 \mathrm{H}), 4.60(\mathrm{~m}, 1.74 \mathrm{H}), 4.51(\mathrm{~m}, 0.16 \mathrm{H}), 2.22(\mathrm{~m}, 2.53 \mathrm{H}), 2.12(\mathrm{~m}, 5.91 \mathrm{H})$, $1.85(\mathrm{~m}, 2.56 \mathrm{H}), 1.73(\mathrm{~m}, 1.82 \mathrm{H}) .{ }^{13} \mathrm{C} \mathrm{NMR}\left(\mathrm{CDCl}_{3}, 300 \mathrm{MHz}\right): \delta 158.4(\mathrm{C}=\mathrm{O}), 146.8(\mathrm{C}-\mathrm{O}$, furan ring) 118.7 (C-C, furan ring) $79.7\left(\mathrm{CH}-\mathrm{OR}\right.$ ring) $73.2(\mathrm{CH}-\mathrm{OH}$ ring $), 41.6\left(\mathrm{CH}_{2}\right.$ ring $), 32.8\left(\mathrm{CH}_{2}\right.$ ring $), 30.3\left(\mathrm{CH}_{2}\right.$ ring).

Trimer 1,3-cyclopentanediol-furanoate, $-30 \%$ cis

The product was obtained as light brown solids $\left(1.37 \mathrm{~g}, 82 \%\right.$ yield). After drying at $110{ }^{\circ} \mathrm{C}$ discoloration occurred, the product was isolated by dissolving the mix in $\mathrm{CHCl}_{3}$, filter out the brown solids, and reduce the $\mathrm{CHCl}_{3}$ in vacuo, to re-obtain the product as light brown solids $\left(0.91 \mathrm{~g}, 54 \%\right.$ yield). ${ }^{1} \mathrm{H} \mathrm{NMR}\left(\mathrm{CDCl}_{3}, 300\right.$ $\mathrm{MHz}): \delta 7.12(\mathrm{~m}, 2 \mathrm{H}), 5.46(\mathrm{~m}, 2.27 \mathrm{H}), 4.60(\mathrm{~m}, 1.74 \mathrm{H}), 4.51(\mathrm{~m}, 0.16 \mathrm{H}), 2.22(\mathrm{~m}, 2.53 \mathrm{H}), 2.12(\mathrm{~m}, 5.91 \mathrm{H})$, $1.85(\mathrm{~m}, 2.56 \mathrm{H}), 1.73(\mathrm{~m}, 1.82 \mathrm{H}) .{ }^{13} \mathrm{C} \mathrm{NMR}\left(\mathrm{CDCl}_{3}, 300 \mathrm{MHz}\right): \delta 158.4(\mathrm{C}=\mathrm{O}), 146.8(\mathrm{C}-\mathrm{O}$, furan ring) 118.7 (C-C, furan ring) $79.7\left(\mathrm{CH}-\mathrm{OR}\right.$ ring) $73.2(\mathrm{CH}-\mathrm{OH}$ ring $), 41.6\left(\mathrm{CH}_{2}\right.$ ring $), 32.8\left(\mathrm{CH}_{2}\right.$ ring $), 30.3\left(\mathrm{CH}_{2}\right.$ ring). 
Trimer 1,3-cyclopentanediol-furanoate, $-40 \%$ cis

The product was obtained as yellow oil $(0.98 \mathrm{~g}, 87 \%$ yield $)$. After drying at $110^{\circ} \mathrm{C}$ discoloration occurred, the product was isolated by dissolving the mix in $\mathrm{CHCl}_{3}$, filter out the brown solids, and reduce the $\mathrm{CHCl}_{3}$ in vacuo, to re-obtain the product as light brown solids $(0.48 \mathrm{~g}, 43 \%$ yield $) .{ }^{1} \mathrm{H} \mathrm{NMR}\left(\mathrm{CDCl}_{3}, 300 \mathrm{MHz}\right): \delta$ $7.12(\mathrm{~m}, 2 \mathrm{H}), 5.46(\mathrm{~m}, 2.27 \mathrm{H}), 4.60(\mathrm{~m}, 1.74 \mathrm{H}), 4.51(\mathrm{~m}, 0.16 \mathrm{H}), 2.22(\mathrm{~m}, 2.53 \mathrm{H}), 2.12(\mathrm{~m}, 5.91 \mathrm{H}), 1.85$ $(\mathrm{m}, 2.56 \mathrm{H}), 1.73(\mathrm{~m}, 1.82 \mathrm{H}) .{ }^{13} \mathrm{C}$ NMR $\left(\mathrm{CDCl}_{3}, 300 \mathrm{MHz}\right)$ : $\delta 158.4(\mathrm{C}=\mathrm{O}), 146.8(\mathrm{C}-\mathrm{O}$, furan ring) $118.7(\mathrm{C}-$ $\mathrm{C}$, furan ring) $79.7\left(\mathrm{CH}-\mathrm{OR}\right.$ ring) $73.2(\mathrm{CH}-\mathrm{OH}$ ring $), 41.6\left(\mathrm{CH}_{2}\right.$ ring $), 32.8\left(\mathrm{CH}_{2}\right.$ ring $), 30.3\left(\mathrm{CH}_{2}\right.$ ring $)$.

\section{Polymerization method}

Small-scale screening in TGA

Trimer pre-polyesters were polymerized in the TGA with or without catalyst. Trimers with catalyst were prepared by dissolving the trimer in $\mathrm{CHCl}_{3}$ followed by the addition of $1 \mathrm{~mol} \%$ catalyst solution $(0.01 \mathrm{M}$ in anhydrous $\mathrm{CHCl}_{3}$ ). Next, the $\mathrm{CHCl}_{3}$ was removed in vacuo, and the trimers with catalyst were stored in a desiccator until use. Polymerization in the TGA was performed by loading 5-10 mg of sample, followed by heating at a rate of $10{ }^{\circ} \mathrm{C} / \mathrm{min}$ to the desired polymerization temperature. Next, the samples were kept isothermal for the desired polymerization time, after which they were cooled to room temperature and used for analysis.

\section{Results and discussion}

\section{Trimer synthesis}

To evaluate the effect of cis/trans ratio in CPdiol, the different cis/trans fractions obtained from fraction distillation were used to synthesize 1,3-cyclopentanediol-furanoate (CP-F) trimers with varying cis content $(8 \%, 20 \%, 30 \%$, and $40 \%)$ via the method described in the previous chapter. Interestingly, with drying the $\mathrm{CP}-\mathrm{F}$ trimers at $110{ }^{\circ} \mathrm{C}$ under vacuum, discoloration occurred, increasing with increasing cis content (shown in the supplementary section for this chapter). To investigate the cause of the increased discoloration the oven-dried samples were analyzed with LC-MS (Figure 2-1). In addition to the standard trimer/pentamer/heptamer distribution, new peaks with a mass difference of -18 , and $-84 \mathrm{~g} / \mathrm{mol}$ were identified. Especially for the pentamer $(n=2)$ distribution, it is clear visible how in increase in cis content leads to an increase in weight loss (inset Figure 2-1). The weight loss of $18 \mathrm{~g} / \mathrm{mol}$ likely corresponds to the occurrence of a dehydration reaction, whereas the weight loss of $84 \mathrm{~g} / \mathrm{mol}$ suggests the liberation of a 3cyclopentenol $(\mathrm{CPol})$ group. To obtain the products in a pure form, the samples were dissolved in chloroform, resulting in a clear solution in combination with black insoluble particles, which were filtered out. Precipitation in heptane yielded the desired products, ranging from off-white to light brown in color. Interestingly, with increasing cis content, the yield dropped from $\sim 80 \%$ to $\sim 50 \%$. Overall, these observations suggest that CPdiol exhibits limited thermal stability, where the cis isomer seems less thermally stable than the trans isomer 


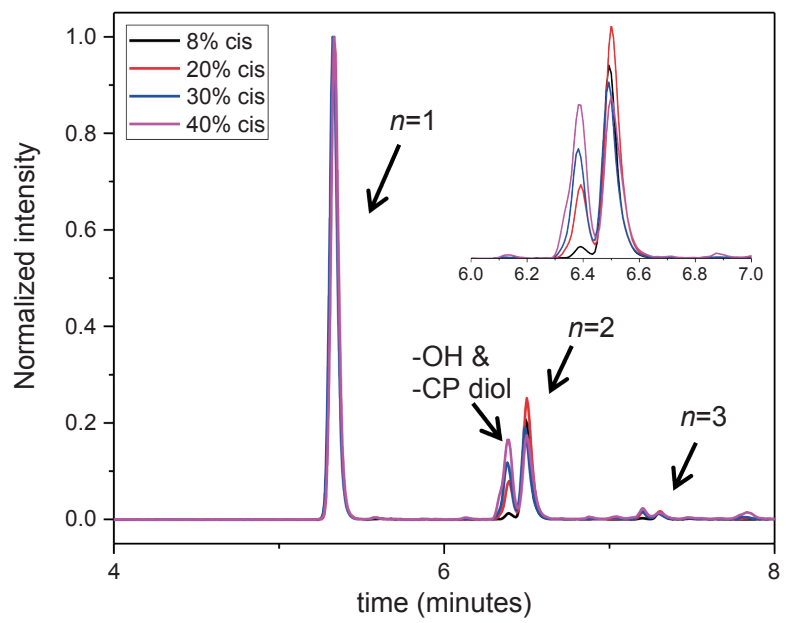

Figure 2-1. LC-MS data on partially degraded CP-F trimers after drying at $110^{\circ} \mathrm{C}$.

\section{Evaluation and optimization of polymerization conditions}

TGA studies were performed to ascertain the right polymerization conditions for each trimer and preventing potential degradation resulting from the thermal instability of the $\mathrm{CP}-\mathrm{F}$ trimers. From the left image in Figure 2-2, it can be observed that the CP-F trimers are all thermally stable up to $200{ }^{\circ} \mathrm{C}$, irrespective of the cis/trans ratio. Considering that no evaporation, sublimation, or degradation occurs below $200^{\circ} \mathrm{C}$, polymerization can be performed in TGA. In this case, all observed mass-loss should result from the generation of the condensate CPdiol: The mass loss at the theoretical maximum conversion equals the mass-loss of one CPdiol group of the trimer, which contributes to $31 \%$ of the weight.
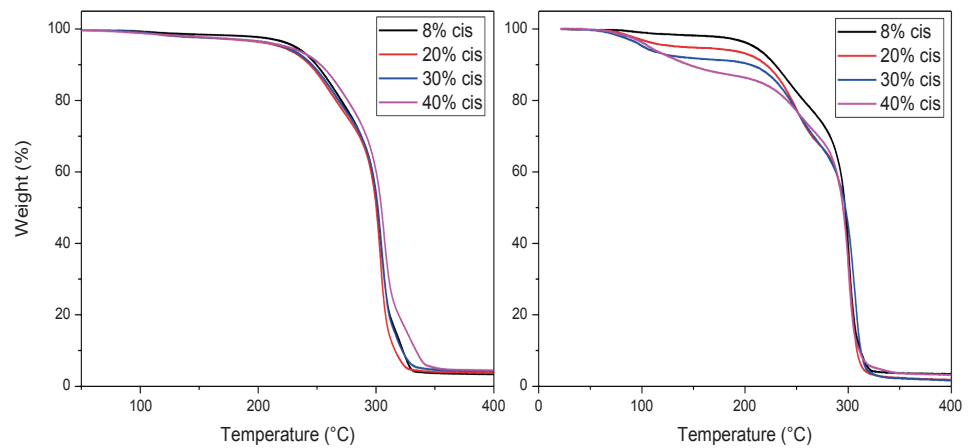

Figure 2-2. TGA experiments for CP-F trimer, with varying cis content, with a heating ramp of $10^{\circ} \mathrm{C} / \mathrm{min}$, non-catalyzed (left) and catalyzed (right).

To this end, all trimers were mixed with $1 \mathrm{~mol} \%$ of Ti(IV)butoxide catalyst and the same TGA experiments were performed after drying (Figure 2-2, right). Indeed, upon the addition of the catalyst, weight loss is found to start around $100{ }^{\circ} \mathrm{C}$, followed by a stable plateau. Further heating results in degradation, starting above $220^{\circ} \mathrm{C}$. The amount of weight-loss between $100{ }^{\circ} \mathrm{C}$ and $200{ }^{\circ} \mathrm{C}$ increases with increasing cis-CPdiol content 
(respectively $3.9,6.8,9.1$, and $13.5 \%$, Figure 2-2, right). These findings indicate that trimers having a higher cis-CPdiol content generate volatiles more rapidly during polymerization, potentially indicating an increased rate of polycondensation. To identify the optimal polymerization temperature, all trimers were polymerized in the TGA for one hour at 180,200 , and $220{ }^{\circ} \mathrm{C}$ (Table 2-1). As observed previously, an increased weightloss is observed with increasing cis-fraction, combined with an increased discoloration of the final product. Due to the absence of a vacuum-step in the polymerization in the TGA, the molecular weight of the resulting oligomers remained low, with $M_{n}$ varying between 500 and $3000 \mathrm{~g} / \mathrm{mol}$. The melting behavior of the polymers was determined via polarized optical microscopy (POM) equipped with a Linkam hot-stage, rather than in DSC due to the limited thermal stability of the samples. The hot-stage was heated at a rate of 10 ${ }^{\circ} \mathrm{C} / \mathrm{min}$, and the visual off-set of melting is listed in Table 2-1.

Table 2-1. Results of small-scale polymerizations performed in TGA.

\begin{tabular}{|c|c|c|c|c|c|c|c|c|c|}
\hline Polymer & Cis & Pol. T. & W. loss & Color & $M_{\mathrm{n}}(\mathrm{g} / \mathrm{mol})$ & $M_{\mathrm{w}}(\mathrm{g} / \mathrm{mol})$ & $\bigoplus$ & $T_{\mathrm{m}}\left({ }^{\circ} \mathrm{C}\right)$ & $T_{\mathrm{d}}\left({ }^{\circ} \mathrm{C}\right)$ \\
\hline$p o l y(C P-F)$ & $8 \%$ & $180^{\circ} \mathrm{C}$ & $11.1 \%$ & light yellow & 1224 & 2448 & 2.0 & 260 & 285 \\
\hline poly $(C P-F)$ & $8 \%$ & $200{ }^{\circ} \mathrm{C}$ & $19.1 \%$ & light orange & 1846 & 3628 & 1.97 & 300 & 308 \\
\hline poly $(C P-F)$ & $8 \%$ & $220^{\circ} \mathrm{C}$ & $26.1 \%$ & light brown & 2769 & 6401 & 2.31 & 285 & 295 \\
\hline poly $(C P-F)$ & $20 \%$ & $180^{\circ} \mathrm{C}$ & $9.2 \%$ & light orange & 546 & 1206 & 2.21 & 290 & 296 \\
\hline poly $(C P-F)$ & $20 \%$ & $200{ }^{\circ} \mathrm{C}$ & $20.3 \%$ & light orange & 1602 & 3484 & 2.17 & 285 & 296 \\
\hline poly $(C P-F)$ & $20 \%$ & $220^{\circ} \mathrm{C}$ & $26.8 \%$ & light brown & 2680 & 6772 & 2.53 & 267 & 281 \\
\hline poly $(C P-F)$ & $30 \%$ & $180^{\circ} \mathrm{C}$ & $11.8 \%$ & light brown & 525 & 1161 & 2.21 & $245-280$ & 280 \\
\hline poly $(C P-F)$ & $30 \%$ & $200{ }^{\circ} \mathrm{C}$ & $23.3 \%$ & light brown & 1660 & 3724 & 2.24 & $245-282$ & 280 \\
\hline poly (C-PF) & $30 \%$ & $220^{\circ} \mathrm{C}$ & $28.9 \%$ & brown & 2342 & 7169 & 3.06 & $254-274$ & 274 \\
\hline poly $(C P-F)$ & $40 \%$ & $180^{\circ} \mathrm{C}$ & $14.2 \%$ & tr. brown & 663 & 1414 & 2.13 & - & n.d. \\
\hline poly $(C P-F)$ & $40 \%$ & $200{ }^{\circ} \mathrm{C}$ & $23.5 \%$ & tr. brown & 2071 & 4503 & 2.18 & - & n.d. \\
\hline poly $(C P-F)$ & $40 \%$ & $220^{\circ} \mathrm{C}$ & $29.1 \%$ & dark brown & 2330 & 8129 & 3.49 & - & n.d. \\
\hline
\end{tabular}

As expected, all samples showed rapid signs of (further) discoloration with the evolution of gasses directly after melting, indicating that the degradation temperature $\left(T_{\mathrm{d}}\right)$ is very close to the melting temperature $\left(T_{\mathrm{m}}\right)$. Additionally, increasing the cis-fraction resulted in an increase in $M_{\mathrm{w}}$ and a decrease in $T_{\mathrm{m}}$, until a fully amorphous material is obtained for the polymer having $40 \%$ cis-CPdiol. These findings are in coherence with literature on the cis/trans effect of other cyclic diols.

The difference in polymerization behavior of varying cis-CPdiol content becomes apparent by analyzing the GPC traces (Figure 2-3). In general, the trimer containing $8 \%$ cis-CPdiol polymerized rapidly at $180{ }^{\circ} \mathrm{C}$, indicated by the narrow $Đ$ and the relatively high molecular weight compared to the polymer with higher cis-CPdiol content. When increasing the reaction temperature to $200{ }^{\circ} \mathrm{C}$, the rise of a high molecular weight tail is observed in the molecular weight distribution. In fact, this is true for all samples, and is particularly dominant in the sample having $40 \%$ cis-CPdiol. Further heating to $220{ }^{\circ} \mathrm{C}$ 
broadens the molecular weight distribution even further, and this effect again becomes more apparent with increasing amount of cis-CPdiol. It should be noted that the oligomers with increasing cis-CPdiol content became increasingly difficulty to dissolve in the HFIP solvent. In fact, especially for the oligomers containing 30 and $40 \%$ cis-CPdiol, black insoluble components remained in the HFIP solvent, which were filtered off prior to GPC analysis. This indicates that the GPC traces shown in Figure 2-3 might not reflect the true polymer content as not all material has dissolved.
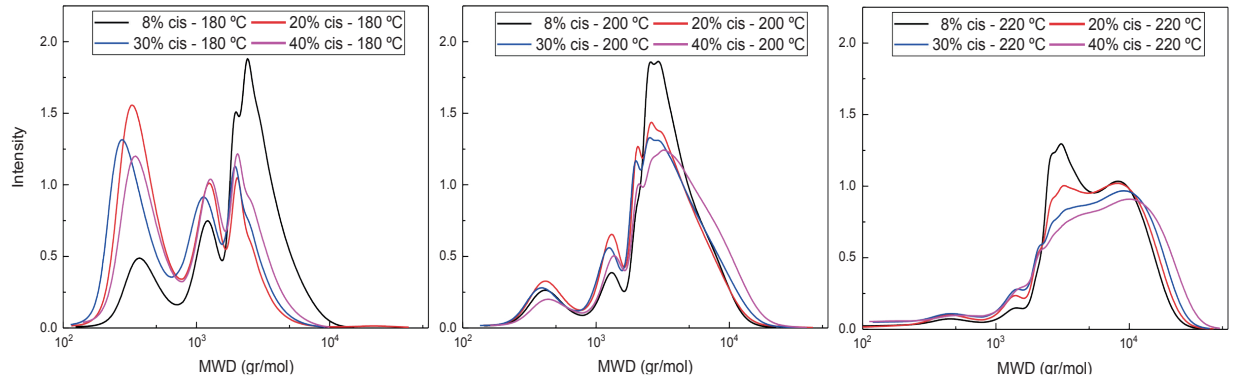

Figure 2-3. GPC traces of poly(CP-F) polymerized at $180{ }^{\circ} \mathrm{C}$ (left), $200{ }^{\circ} \mathrm{C}$ (middle), and $220{ }^{\circ} \mathrm{C}$ (right), with varying cis-1,3-CPdiol content from $8 \%$ (black), $20 \%$ (red), $30 \%$ (blue) to $40 \%$ (purple).

Nevertheless, the formation of insoluble polymer particles suggests the presence of a cross-linking side reaction: The presence of a bimodal distribution is highly unlikely in polycondensation reactions considering the continuously occurring transesterification reaction in combination with Flory's equireactivity theory. Instead, considering the increasing discoloration and decreasing solubility of polymers with increasing cis-CPdiol content, we consider it more likely that cis-CPdiol undergoes a degradative cross-linking reaction in addition to the polycondensation reaction, thereby generating high molecular weight components.

\section{Oligomer analysis using MALDI-ToF-MS}

Assuming that the CP-F polycondensation reaction proceeds without side-reactions, only the presence of linear chains with cyclopentanol ( $\mathrm{CPol}$ ) groups on both chain ends is expected (structure 1, Figure 2-4). As is observed from LC-MS analysis, thermal dehydration $(2,3)$, and loss of $1,3-\mathrm{CPol}$ end-group $(4,5)$ can be expected to occur as a side-reaction during polymerization. To assess whether such a side reaction proceeds during polymerization, the obtained oligomers have been analyzed using MALDI-TOFMS.

Exemplified in Figure 2-5 are the MALDI-ToF-MS spectra for poly(CP-F) with $8 \%$ cis, polymerized at $180^{\circ} \mathrm{C}$ (top, blue), $200^{\circ} \mathrm{C}$ (middle, red) and $220^{\circ} \mathrm{C}$ (bottom, black). The vertical solid lines are the calculated masses for poly(CP-F) (1, Figure 2-4) with repeat units $n=2$ at the left $(\mathrm{m} / \mathrm{z}=807.2)$ to $n=7$ at the right $(\mathrm{m} / \mathrm{z}=1917.7) .8 \%$ cis polymerized at $180{ }^{\circ} \mathrm{C}$ shows a neat distribution of the polymer. Though when polymerized at $200{ }^{\circ} \mathrm{C}$, some new distributions are coming up. This is especially clear for the polymerization of 


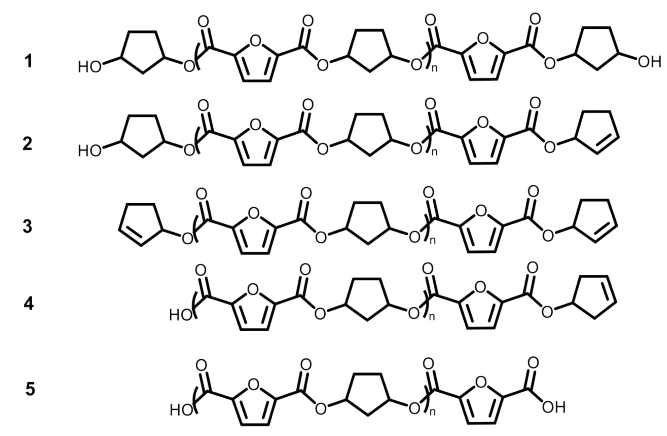

Figure 2-4. Expected chemical structures of linear chain products for poly(CP-F) before and after thermal stability experiments.

$8 \%$ cis at $220{ }^{\circ} \mathrm{C}$ where the main distribution is nearly gone, and two new main distributions are showing with a mass difference of 18 and 36 dalton, similar to the LCMS data shown in Figure 2-1. The calculated masses correspond well with the expected linear chain distribution with partial or full dehydration of 1,3-CPdiol end-groups as depicted in Figure 2-4 (2 and 3, respectively). The full MALDI-ToF-MS spectra for poly(CP-F) with 20,30 , and $40 \%$ cis polymerized at the same temperatures are shown in the supplementary information for this chapter.

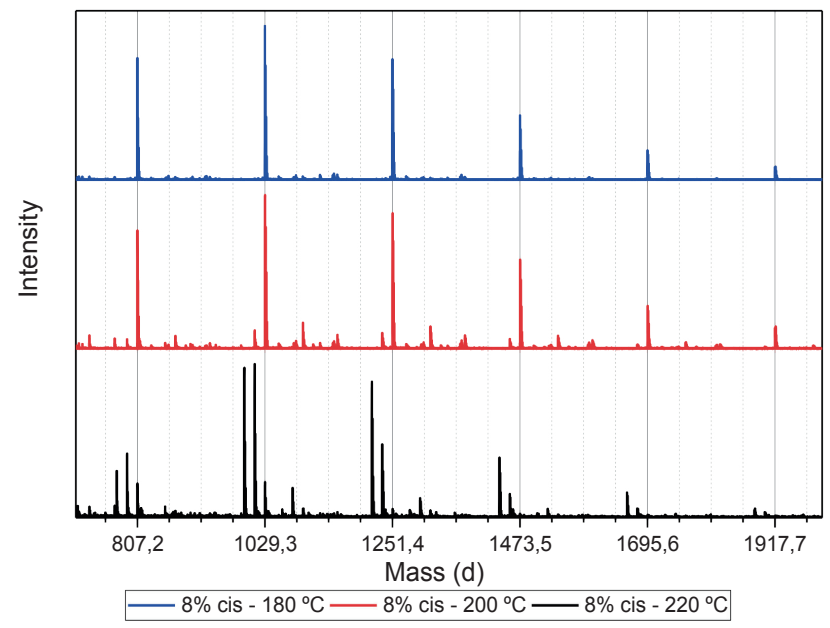

Figure 2-5. MALDI-ToF-MS spectra for poly(CP-F) with $8 \%$ cis polymerized at $180{ }^{\circ} \mathrm{C}$ (top), $200{ }^{\circ} \mathrm{C}$ (middle), and $220^{\circ} \mathrm{C}$ (bottom). The calculated masses for poly(CP-F) with $n$ repeating units is shown by the vertical solid lines, starting from $n=2$ at the left $(\mathrm{m} / \mathrm{z} 807.2)$ to $n=7$ at the right $(\mathrm{m} / \mathrm{z} 1917.7)$.

To properly assess the new distributions per polymer, and assess the effect of the increasing amount of cis in the different trimers, we focus on the change in distribution in between repeat units $n=2(\mathrm{~m} / \mathrm{z}$ 807.2) and $n=3(\mathrm{~m} / \mathrm{z} 1029.0)$. To this end, the mass distributions of the oligomers having $8 \%$ (Figure 2-6, left), 20\% (Figure 2-6, right), 30\% (Figure 2-7, left), and 40\% cis-CPdiol (Figure 2-7, right) after polymerization at $180{ }^{\circ} \mathrm{C}$ (blue, top), $200{ }^{\circ} \mathrm{C}$ (red, middle), and $220^{\circ} \mathrm{C}$ (black, bottom) are shown. 
Figure 2-6, left, shows the same single distribution of poly(CP-F) with $8 \%$ cis-CPdiol polymerized at $180^{\circ} \mathrm{C}$ (blue), which corresponds to the expected linear polyester 1 (as is noted on top of the figure). An increase in polymerization temperature leads to dehydration of the alcohol end-group of CPdiol, leading to a rise of products $\mathbf{2}$ and $\mathbf{3}$ at the expense of product $\mathbf{1}$, as is clearly visible from the MALDI-ToF-MS spectrum taken at $220{ }^{\circ} \mathrm{C}$ (black). Similar to the samples having $8 \%$ cis-CPdiol, an increase in polymerization temperature for the sample having $20 \%$ cis-CPdiol results in dehydration of the CPdiol end-groups, as is indicated by the rise in reaction products $\mathbf{2}$ and $\mathbf{3}$.

Interestingly, the presence of product 1 becomes less abundant at $180{ }^{\circ} \mathrm{C}$ as the cis content increases. Instead, the samples with $20 \%, 30 \%$, and $40 \%$ cis-CPdiol polymerized at $180{ }^{\circ} \mathrm{C}$ contain products corresponding to chains with one or two carboxylic acid endgroups (structure 4 and 5 respectively, Figure 2-4). Since the carboxylic acid moiety is not present as starting material, they have to be generated during polymerization. Generally, carboxylic acid groups can be generated through ester hydrolysis by water, (degradative) chain-scission of ester bonds, or complete removal of a 3-cyclopentenol end-group. ${ }^{11,12}$ Hydrolysis by water would lead to a distribution of chains with a CPol endgroup on one side, and a carboxylic acid on the other side. However, the corresponding mass distribution was not detected in MALDI-TOF-MS analysis. Instead, (degradative) chain-scission of ester bonds would lead to product $\mathbf{4}$ and $\mathbf{5}$, however would also inherently lead to a drop in molecular weight, which was not observed in GPC analysis (Figure 2-3). Thus it seems likely that only the CPol end-groups have been removed from the product, leading to the observed drop in product $\mathbf{1}$, and rise in the carboxylic acid terminated products $\mathbf{4}$ and $\mathbf{5}$.

An increase in polymerization temperature from $180{ }^{\circ} \mathrm{C}$ to $200{ }^{\circ} \mathrm{C}$ and $220{ }^{\circ} \mathrm{C}$ for $\mathrm{CP}-\mathrm{F}$ samples having $20 \%, 30 \%$, and $40 \%$ cis-CPdiol results in a decrease in distributions 4 and 5 . This could potentially be resulting from thermal decarboxylation of the generated FDCA moieties ${ }^{11,12}$, which yields a mono-substituted furan based diene, which can undergo a Diels-Alder reaction with CPene end-groups, also providing a potential route

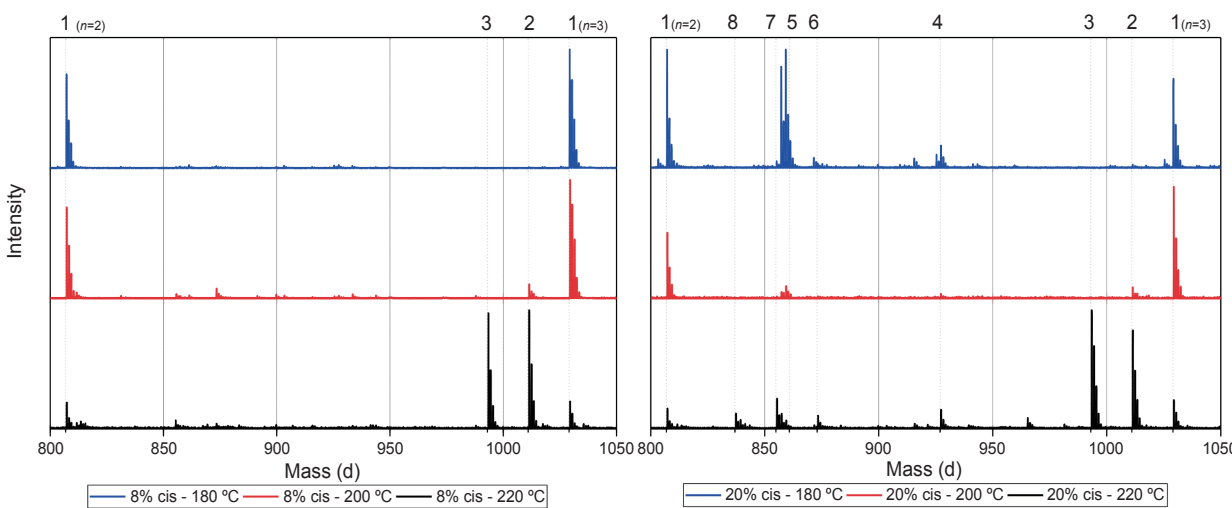

Figure 2-6. MALDI-ToF-MS spectra of poly(CP-F) polymerized at $180^{\circ} \mathrm{C}$ (blue, top), $200{ }^{\circ} \mathrm{C}$ (red, middle), and $220^{\circ} \mathrm{C}$ (black, bottom), with cis fractions of $8 \%$ (left), and $20 \%$ (right). Zoomed in on $750-1050$ Dalton, between repeating units $n=2(m / z=806.82)$ and $n=3(m / z=1029.02)$ of linear diol 1. 
towards branched or cross-linked species. We will elaborate further on this hypothesis later in this work.

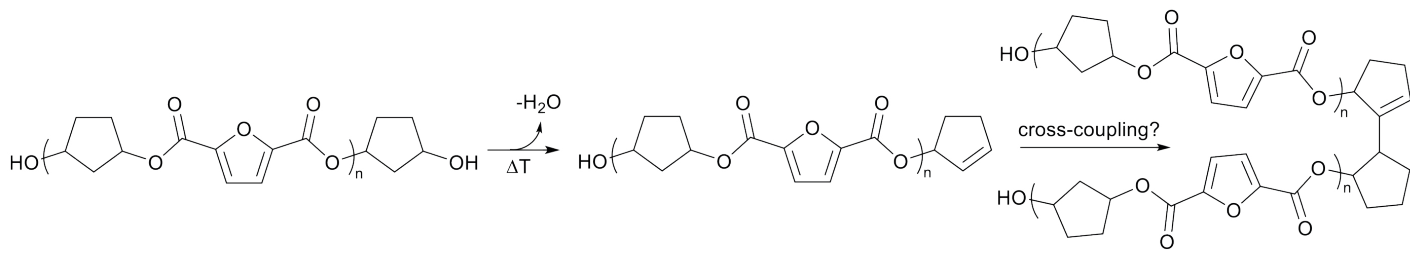

Scheme 2-1. Anticipated degradation process of oligomers. A cyclopentanol end-group can undergo thermal dehydration, generating a cyclopentene end-group, which in turn can undergo a cross-coupling reaction.

To recall, both LC-MS (provided in the SI) and the MALDI-TOF-MS analysis depicted in

Figure 2-5 suggest that thermal dehydration takes place on the external $\mathrm{OH}$ group of CPdiol, thereby generating cyclopentene end-groups. These cyclopentene groups are likely to undergo cross-coupling reactions (Scheme 2-1), to form a Cp-Cp coupled linear chain with CPdiol end-groups (Scheme 2-1, right) which mass is found at distribution marked 6. In turn, these end-groups are susceptible to single and double dehydration to lead to distributions marked $\mathbf{7}$ and $\mathbf{8}$, respectively (structures are shown in the supplementary section for this chapter). Though this CPene-CPene cross-coupling provides a means to build up higher molecular weight materials, it is considered a sidereaction of the dehydration of mostly cis-CPdiol end-groups at elevated temperatures. This is supported by the observed rise in 6, 7, and 8 in the samples with increasing cisCPdiol content, and with increasing temperatures, confirming that these cross-coupling reactions are occurring. This CPene-CPene cross-coupling reaction also explain the rise in molecular weight observed in GPC analysis of samples having higher cis-CPdiol content (Figure 2-3).
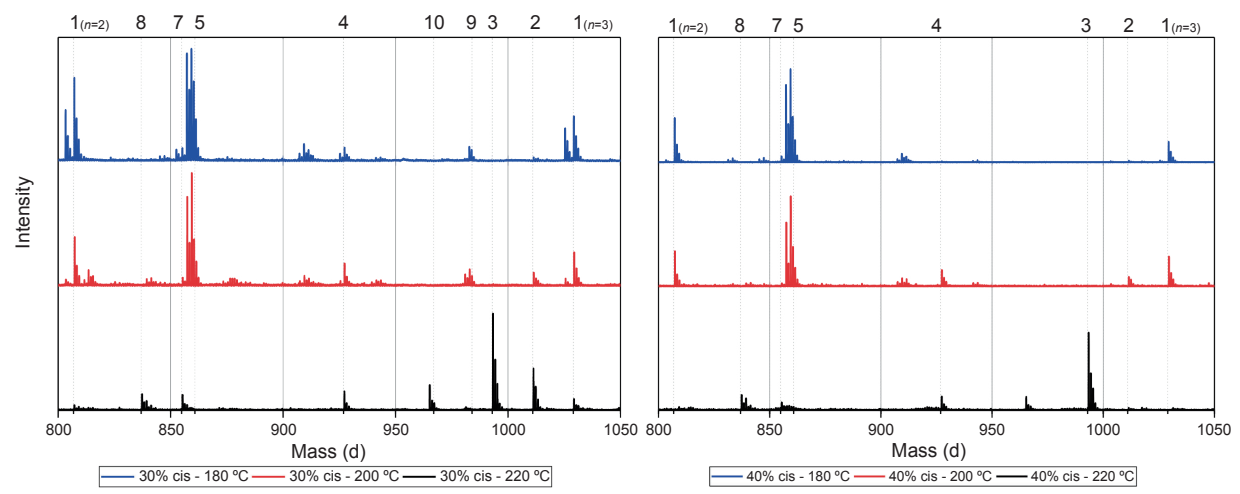

Figure 2-7. MALDI-ToF-MS spectra of poly(CP-F) polymerized at $180{ }^{\circ} \mathrm{C}$ (blue, top), $200{ }^{\circ} \mathrm{C}$ (red, middle), and $220^{\circ} \mathrm{C}$ (black, bottom), with cis fractions of $30 \%$ (left), and $40 \%$ (right). Zoomed in on $750-1050$ Dalton, between repeating units $n=2(m / z=806.82)$ and $n=3(m / z=1029.02)$ of linear diol 1. 
As indicated earlier, an additional chain-extension reaction that can take place is the Diels-Alder addition reaction between decarboxylated furan moieties and CPene groups. This Diels-Alder side reaction would result into the formation of distribution 9, as shown in Scheme 2-2. Indeed, such a distribution is found in the polymer having $30 \% \mathrm{cis}-\mathrm{CPdiol}$, (highlighted by dotted line 9 in Figure 2-7, left). With increased polymerization temperature to $220{ }^{\circ} \mathrm{C}$, distribution 9 decreases, and a distribution with a mass loss of 18 $\mathrm{g} / \mathrm{mol}$ arises (dotted line 10, Figure 2-7). It is likely that distribution 10 corresponds to the dehydrated product of $\mathbf{9}$. In fact, this distribution is observed in both polymers having $20 \%$ and $40 \%$ cis-CPdiol, polymerized at $220^{\circ} \mathrm{C}$. These findings provide further proof for the occurrence of a Diels-Alder reaction between the degradation products.

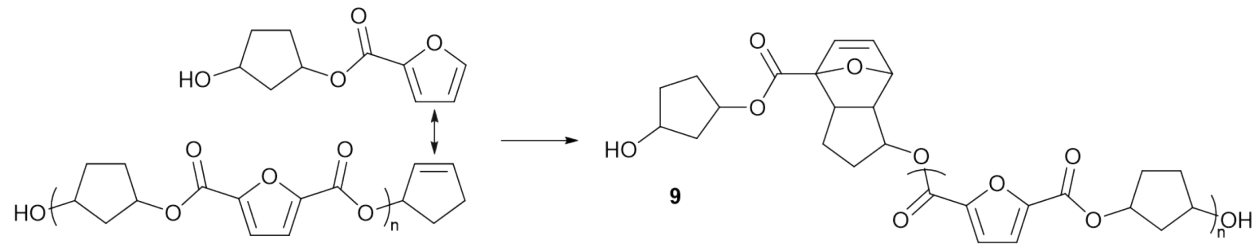

Scheme 2-2. Anticipated Diels-Alder side reaction between a furan group and a CPene group leading to a polymer distribution $\mathbf{9}$.

It should be noted that the obtained mass-distributions in these MALDI-ToF-MS analysis seem limited to fit only linear chains that are ending with either carboxylic acid, CPol, or CPene end-groups. Although cross-linked or branched mass-distributions are expected given the presence of insoluble components in GPC analysis, such cross-linked or branched fractions are not detected in these MALDI-ToF-MS experiments. Possibly these branched or cross-linked polymers are filtered off during sample preparation or are too low in concentration to measure. Nevertheless, the current MALDI-ToF-MS data provides valuable insights in the polymerizability and thermal stability of CPdiol as monomer in polycondensation reactions.

\section{Stability difference cis- and trans-1,3-cyclopentanediol}

The obtained results in the polymerization stability study suggest that there is an inherent difference in thermal stability between the cis and trans isomer of 1,3-cyclopentanediol: Structures with mostly trans-CPdiol end-groups undergo thermal dehydration at temperatures of $200{ }^{\circ} \mathrm{C}$ and higher, thereby generating CPene end-groups. Structures with cis-CPdiol end-

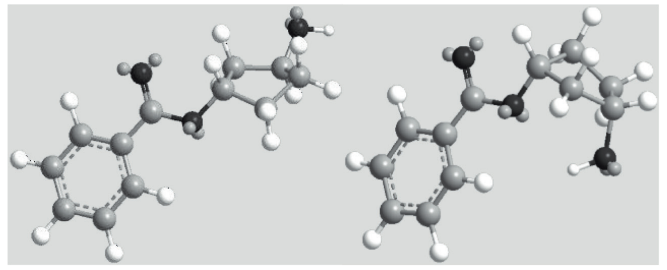

Figure 2-8. Minimized energy calculations of the mono-benzoate ester of trans-CPdiol (left) and cisCPdiol (right). Calculated with Chemdraw 3D v17, via MM2 molecular dynamics and minimize energy. 
groups rather undergo degradative ester bond cleavage at $180{ }^{\circ} \mathrm{C}$ instead, thereby generating free carboxylic acid groups in addition to CPene end-groups. It is possible that the origin of this stability difference can be found in the different conformations of these two isomers. The conformations of cis- and trans-1,3-cyclopentanediol have been evaluated using theoretical and NMR investigations in the work of Koniotou et al. ${ }^{10}$ These authors reported that the most stable conformers for cis-CPdiol have the hydroxyl groups in axial positions, allowing for intramolecular hydrogen bonding. ${ }^{13}$ In contrast, the most stable conformers for trans-CPdiol have the hydroxyl groups in equatorial positions, where intramolecular hydrogen bonding is not observed. When these findings are extrapolated to a benzylester of 1,3-cyclopentanediol, preliminary minimized energy calculations in ChemDraw 3D software show similar axial and equatorial positioning (Figure 2-8). These conformations possibly allow cis-CPdiol to undergo intramolecular hydrogen bonding of the external hydroxyl group with the ester bond on the same CP-ring (Scheme 2-3, left).

Combining our experimental findings with the preferred conformations of CPdiol esters, suggests that the origin in thermal stability between cis- and trans-CPdiol might be related to the absence or presence of intramolecular hydrogen bonding. Even though in-depth mechanistic studies of the degradation are beyond the scope of this research, a possible degradation mechanism based on the conformations of CPdiol is postulated in Scheme 2-3. In both cases, $\beta$-hydrogen elimination takes place, followed by the breaking of a $\sigma$-bond: either $\mathrm{C}-\mathrm{OR}$ (left), or $\mathrm{C}-\mathrm{OH}$ (right). Without intramolecular hydrogen bonding the weakest $\sigma$-bond to break is $\mathrm{C}-\mathrm{OH}$, leading to dehydration in the trans isomers (Scheme 2-3, left). However, with intramolecular hydrogen bonding it appears that C-OR becomes the weaker $\sigma$-bond, leading to removal of the CPol group, generation a carboxylic acid-terminated chain (Scheme 2-3, right).

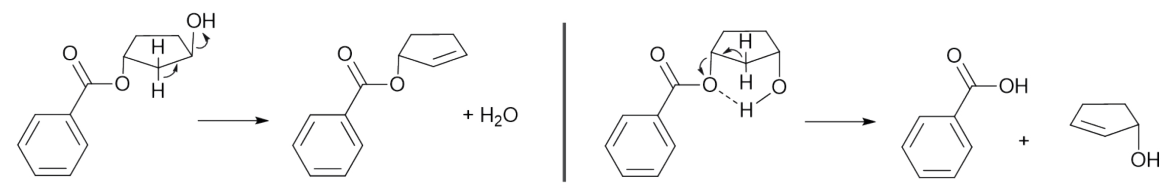

Scheme 2-3. Proposed thermal $\beta$-hydrogen elimination mechanisms, followed by cleavage of a $\sigma$-bond of a mono-benzoate ester of trans- (left) and cis- (right) 1,3-CPdiol.

Furthermore, dehydration via $\beta$-hydrogen elimination is acid catalyzed, and indeed it is observed that with increasing acid-terminated species 5, dehydrated species (2 and 3) start to form at the expense of diol terminated chains (1) (Figure 2-6 and Figure 2-7). Therefore, it appears that the thermal degradation mechanism of cis-1,3-CPdiol leads to free acid species, which in turn catalyzes the dehydration of the remaining trans 1,3-CPdiol end-groups, leading to an even higher depolymerization rate and hence a lower thermal stability of the polymer. Please note that there is no increase in the low 
molecular weight fractions observed at the evaluated polymerization temperatures (Figure 2-3), indicating that the difference in thermal stability at these temperatures seems to originate solely from the difference in cis or trans end-groups. The thermal stability seems enhanced once the 1,3-CPdiol end-groups are completely polymerized even though 'normal' $\beta$-hydrogen chain scission still occurs at elevated temperatures. ${ }^{12}$ Unfortunately, we were unable to study the rate difference in $\beta$-hydrogen chain scission between the cis and trans conformers of CPdiol. This is related to the inability of the poly(CP-F) to build up high molecular weight under the explored polymerization conditions.

\section{Conclusions}

The effect of the cis/trans ratio of 1,3-cyclopentanediol on the polymer properties have been studied on oligomers of CPdiol and FDCA poly(CPF) with cis-CPdiol contents of 8 , 20,30 , and $40 \%$. No apparent effect on the $T_{\mathrm{g}}$ was measured, which agrees with the theoretical similar half-boat confirmation of both isomeric cyclopentane rings. However, with increasing cis-content the crystallization is hampered, and crystallinity fully disappears with $40 \%$ cis-CPdiol content, which displays typical behavior for racemic cyclic diols in polyesters. Combined analysis of TGA, GPC, and MALDI-ToF-MS showed that polyesters having mostly trans-CPdiol isomer are found to be stable up to $180{ }^{\circ} \mathrm{C}$, and undergo dehydration at the CPdiol end-groups at $200{ }^{\circ} \mathrm{C}$ and higher. This dehydration leads to CPene end-groups with an unsaturation, which could undergo cross-coupling reactions at higher temperatures, which was confirmed by MALDI-ToFMS analysis. In contrast, esters having more of cis-1,3-CPdiol isomer are thermally labile, as they undergo rapid degradation already at $180^{\circ} \mathrm{C}$. Instead of thermal dehydration, the entire cyclopentenol end-group is cleaved, most probable via the $\beta$-hydrogen chainscission mechanism, generating polyesters with free acid end-groups already at $180^{\circ} \mathrm{C}$ and $200{ }^{\circ} \mathrm{C}$. In turn, these free acid end-groups further promote both degradation processes of the remaining polymer chains. (Biobased) production of 1,3-cyclopentanediol will always lead to a racemic mixture of the two isomers, similar to the production of other relevant cyclic diols. However, since these findings indicate that cis-1,3-cyclopentanediol is not suitable as building block for (semi-crystalline) polyesters for high-temperature applications, 1,3-cyclopentanediol seems unsuitable as biobased replacement for the established cyclic diols.

\section{Acknowledgements}

This work was performed under the framework of Chemelot InSciTe and is supported by contributions from the European Regional Development Fund (ERDF) within the framework of OP-Zuid and with contributions from the province of Brabant and Limburg and the Dutch Ministry of Economy. 


\section{References}

1. Li, G. et al. Industrially scalable and cost-effective synthesis of 1,3-cyclopentanediol with furfuryl alcohol from lignocellulose. Green Chem. 18, 3607-3613 (2016).

2. Wang, J. et al. Modification of poly(ethylene 2,5-furandicarboxylate) (PEF) with 1, 4-cyclohexanedimethanol: Influence of stereochemistry of 1,4-cyclohexylene units. Polymer (Guildf). 137, 173-185 (2018).

3. Celli, A. et al. Effect of 1,4-cyclohexylene units on thermal properties of poly(1,4-cyclohexylenedimethylene adipate) and similar aliphatic polyesters. Polym. Int. 62, 1210-1217 (2013).

4. Kibler, C. J., Bell, A. \& Smith, J. G. Polyesters of 1,4-cyclohexanedimethanol1. J. Polym. Sci. Part A Gen. Pap. 2, 21152125 (1964).

5. Colonna, M. et al. Poly(1,4-cyclohexylenedimethylene-1, 4-cyclohexanedicarboxylate): analysis of parameters affecting polymerization and cis-trans isomerization. Polym. Int. 60, 1607-1613 (2011).

6. Berti, C. et al. Environmentally Friendly Copolyesters Containing 1,4-Cyclohexane Dicarboxylate Units, 1-Relationships Between Chemical Structure and Thermal Properties. Macromol. Chem. Phys. 211, 1559-1571 (2010).

7. Zhang, M., Moore, R. B. \& Long, T. E. Melt transesterification and characterization of segmented block copolyesters containing 2,2,4,4-tetramethyl-1,3-cyclobutanediol. J. Polym. Sci. Part A Polym. Chem. 50, 3710-3718 (2012).

8. Kelsey, D. R., Scardino, B. M., Grebowicz, J. S. \& Chuah, H. H. High impact, amorphous terephthalate copolyesters of rigid 2,2,4,4-tetramethyl-1,3-cyclobutanediol with flexible diols. Macromolecules 33, 5810-5818 (2000).

9. Sandhya, T. E., Ramesh, C. \& Sivaram, S. Copolyesters Based on Poly(butylene terephthalate)s Containing Cyclohexyl and Cyclopentyl Ring: Effect of Molecular Structure on Thermal and Crystallization Behavior. Macromolecules 40, 69066915 (2007).

10. Abraham, R. J. \& Koniotou, R. Conformational analysis. Part 40: a theoretical and NMR investigation of the conformations ofcis- andtrans-cyclopentane-1,3-diol. Magn. Reson. Chem. 41, 1000-1008 (2003).

11. Tsanaktsis, V. et al. Thermal degradation kinetics and decomposition mechanism of polyesters based on 2,5furandicarboxylic acid and low molecular weight aliphatic diols. J. Anal. Appl. Pyrolysis 112, 369-378 (2015).

12. Terzopoulou, Z . et al. Thermal degradation of biobased polyesters: Kinetics and decomposition mechanism of polyesters from 2,5-furandicarboxylic acid and long-chain aliphatic diols. J. Anal. Appl. Pyrolysis 117, 162-175 (2016).

13. Saegebarth, K. Notes: The Bishydroboration of Cyclopentadiene. J. Org. Chem. 25, 2212-2213 (1960). 


\section{Supplementary information for chapter 2}

\section{LC-MS data}

The trimers of $1,3-\mathrm{CP}-\mathrm{F}$ with various cis content were dried at $110{ }^{\circ} \mathrm{C}$, after which significant discoloration was visible (Figure S2 - 1) The obtained trimers were analyzed via LC-MS (Figure 2-1) to assess the degradation products (Table S2 -1) prior to a second purification step.

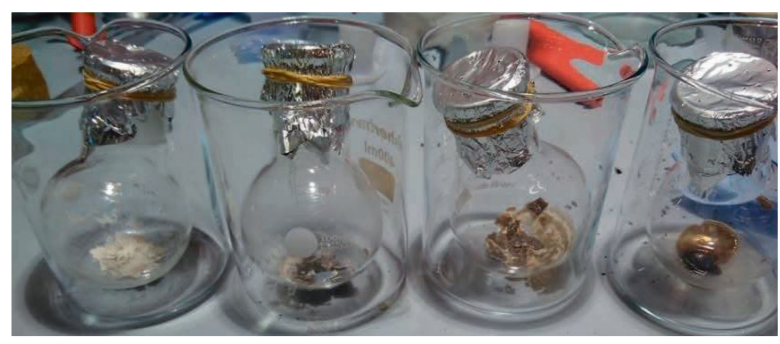

Figure S2 - 1. Obtained trimers of 1,3-CP-F, with varying cis content $(8 \%, 20 \%, 30 \%, 40 \%$, left to right), after drying at $110^{\circ} \mathrm{C}$, showing various stages of discoloration.

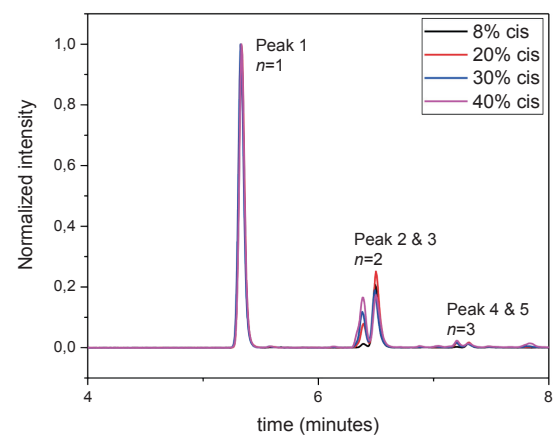

Figure S2 - 2. LC-MS data on partially degraded CP-F trimers after drying at $110^{\circ} \mathrm{C}$.

Table S2 -1. List of masses found for partially degraded 1,3-CP-F trimers in LC-MS, supporting Figure 2-1 and Figure S2 - 2.

\begin{tabular}{|c|c|c|c|}
\hline & $n=1$ & $n=2$ & $n=3$ \\
\hline $\begin{array}{c}\text { Calculated } \\
\text { mass }\end{array}$ & 324.3 & $\begin{array}{l}546.5 \\
528.5(-\mathrm{OH}) \\
462.4(-\mathrm{CPol})\end{array}$ & $\begin{array}{l}768.7 \\
750.7(-\mathrm{OH}) \\
684.6(-\mathrm{CPol})\end{array}$ \\
\hline Peak 1 & $342\left(\mathrm{H}_{3} \mathrm{O}^{+}\right.$adduct $)$ & & \\
\hline Peak 2 & & $463,529,547$ & \\
\hline Peak 3 & & $547,564\left(\mathrm{H}_{3} \mathrm{O}^{+}\right.$adduct $)$ & \\
\hline Peak 4 & & & $685,751,769$ \\
\hline Peak 5 & & & $769,786\left(\mathrm{H}_{3} \mathrm{O}^{+}\right.$adduct $)$ \\
\hline
\end{tabular}


NMR analysis of trimer 1,3-cyclopentanediol-furanoate with changing cis content

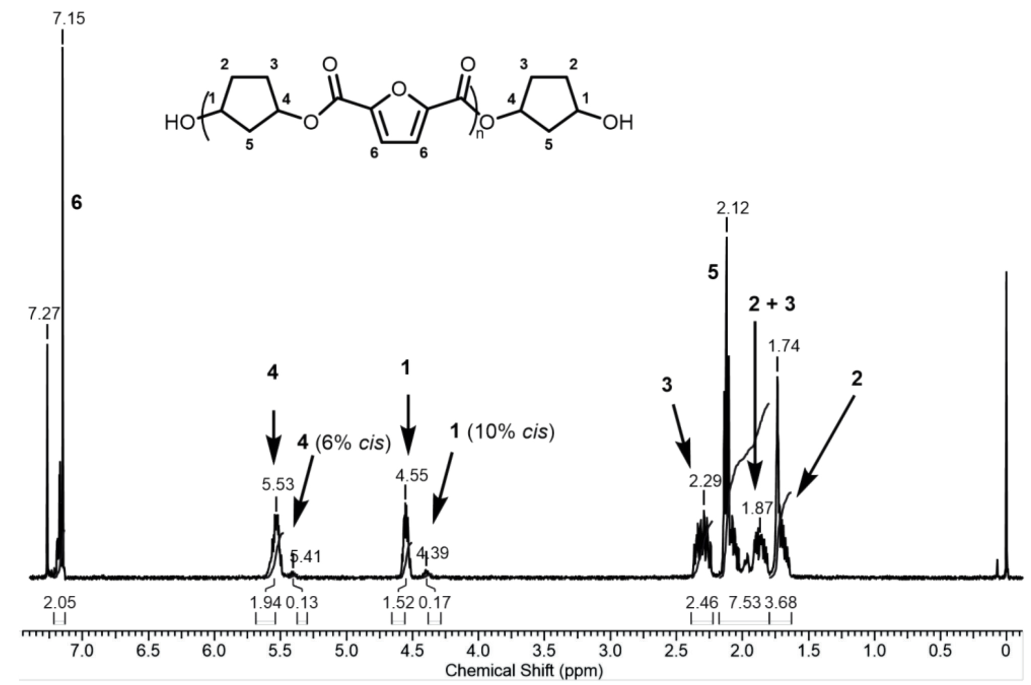

Figure S2 - 3. ${ }^{1} \mathrm{H}-\mathrm{NMR}$ analysis of trimer 1,3-cyclopentanediol-furanoate $-8 \%$ cis.

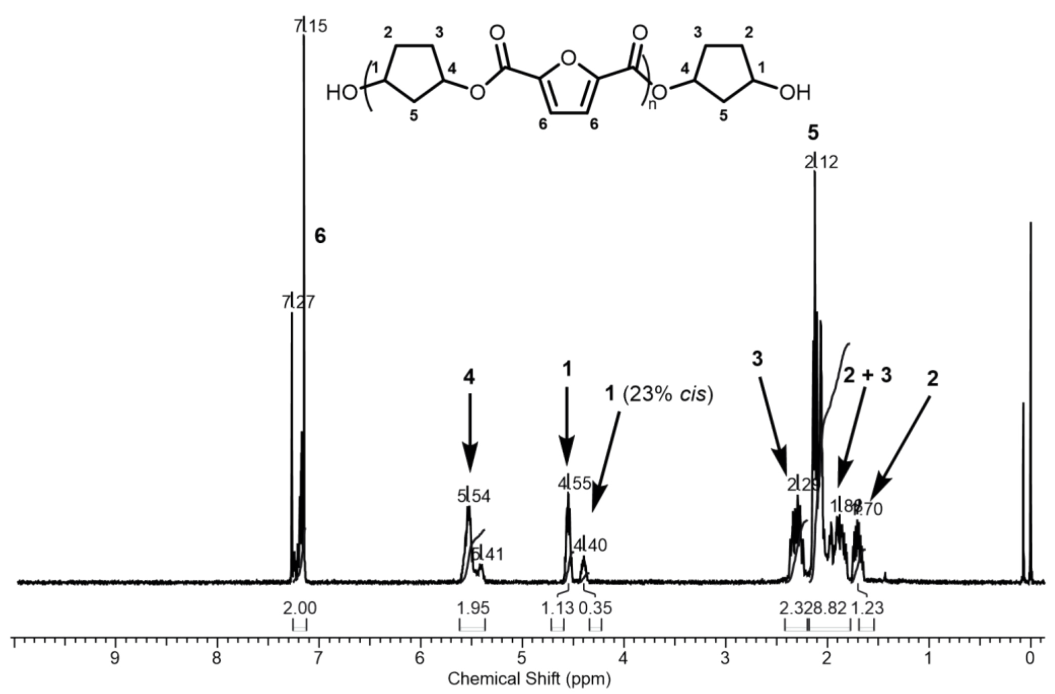

Figure S2 - 4. ${ }^{1} \mathrm{H}-\mathrm{NMR}$ analysis of trimer 1,3-cyclopentanediol-furanoate $-20 \%$ cis. 


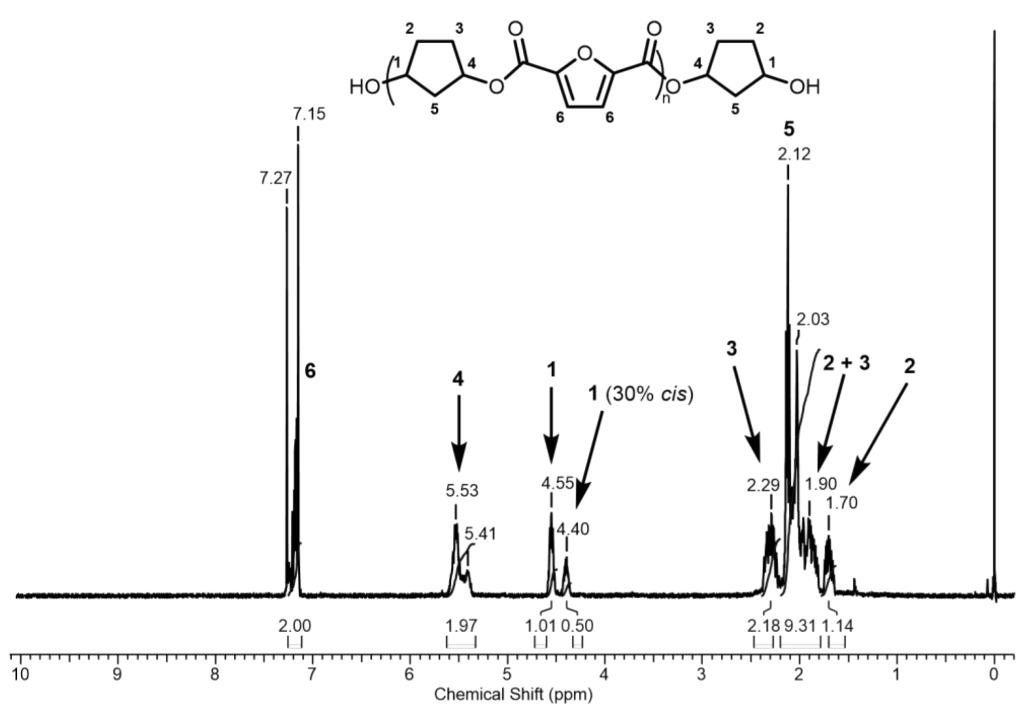

Figure S2 - 5. ${ }^{1} \mathrm{H}-\mathrm{NMR}$ analysis of trimer 1,3-cyclopentanediol-furanoate $-30 \%$ cis.

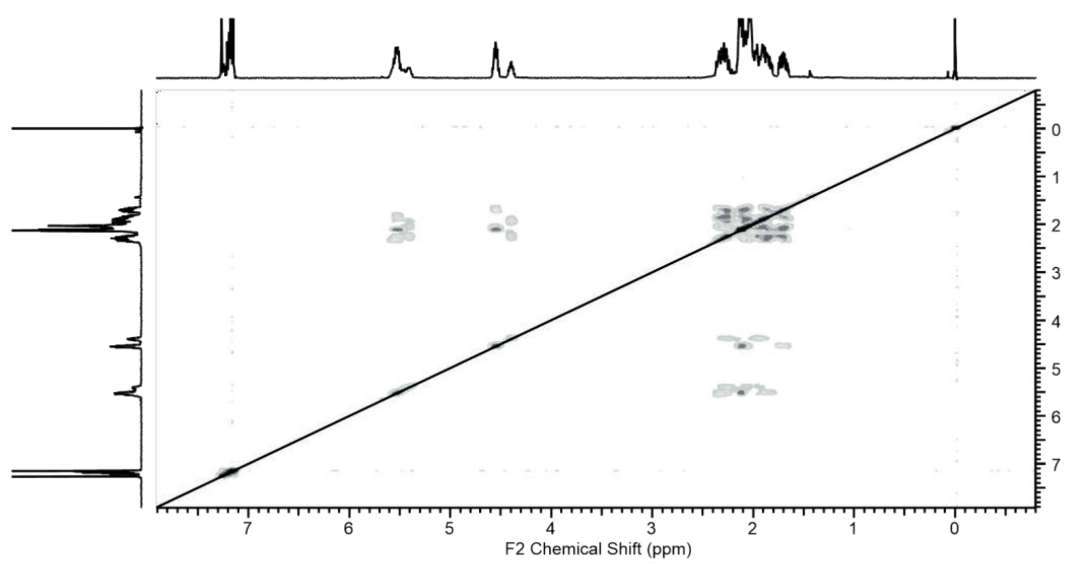

Figure S2 - 6. COSY $\left({ }^{1} \mathrm{H}-{ }^{1} \mathrm{H}\right)$ NMR analysis of trimer 1,3 -cyclopentanediol-furanoate $-30 \%$ cis. 


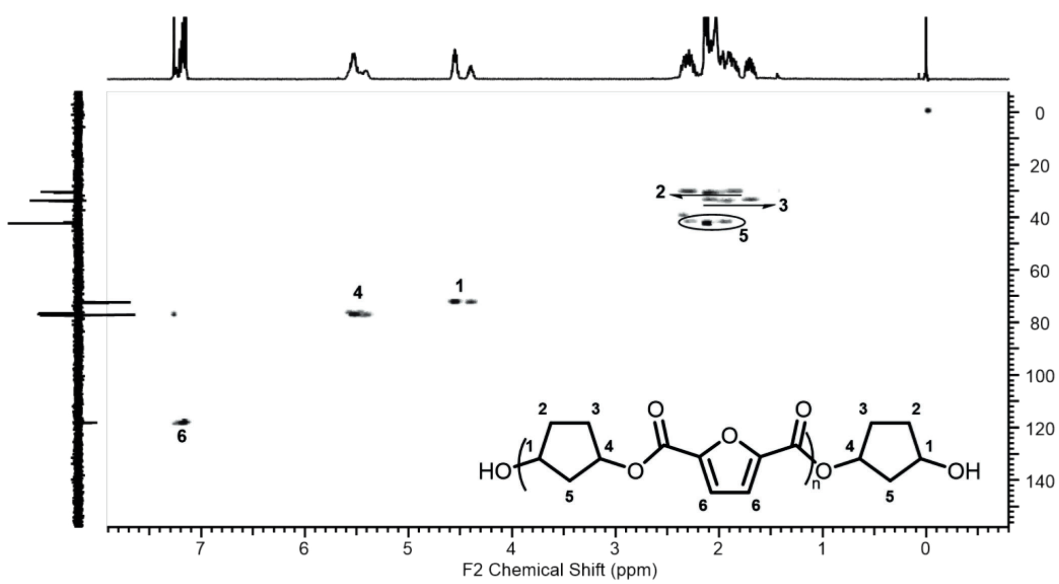

Figure S2 - 7. HSQC ( ${ }^{1} \mathrm{H}$-DEPT) NMR analysis of trimer 1,3-cyclopentanediol-furanoate $-30 \%$ cis.

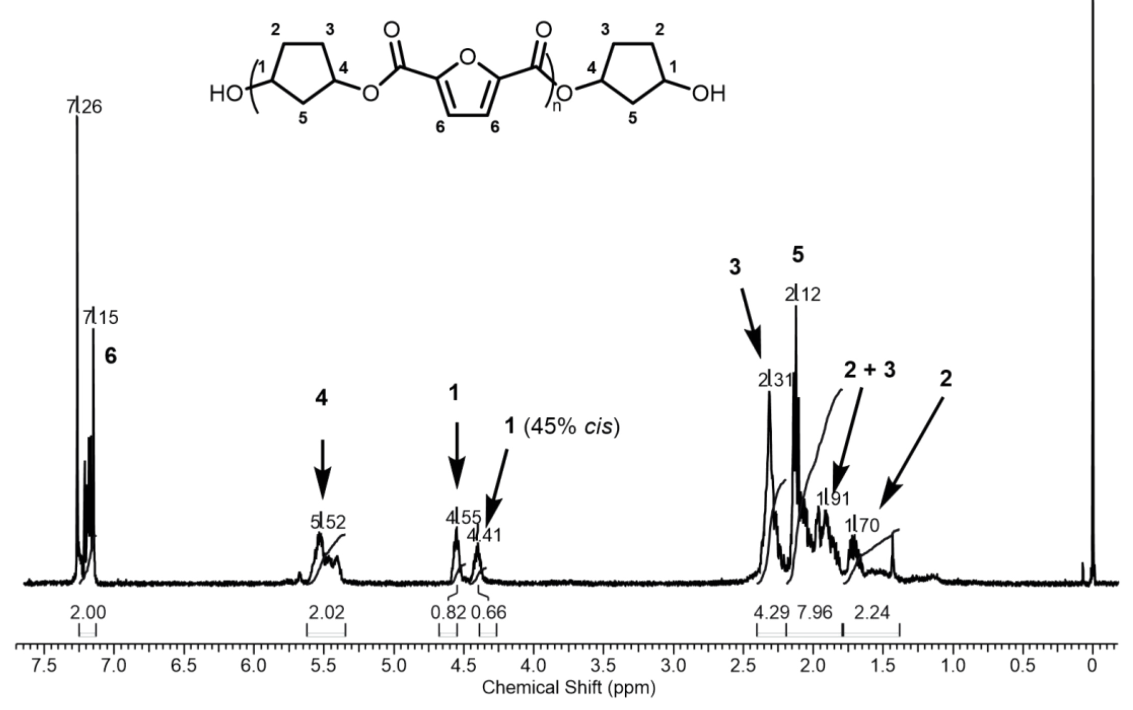

Figure S2 - 8. ${ }^{1} \mathrm{H}-\mathrm{NMR}$ analysis of trimer 1,3 -cyclopentanediol-furanoate $-40 \%$ cis. 


\section{MALDI-ToF-MS analysis}

\section{MALDI-ToF-MS expected linear chains}

The expected linear distributions for poly(1,3-CP-F) are cyclics (not shown) and linear diol terminated chains (01). Thermal dehydration leads to loss of hydroxyl groups (02, and 03). Further degradation can lead to loss of CPol group (04), combined with dehydration (05), eventually leading to di-acid terminated chains (06).

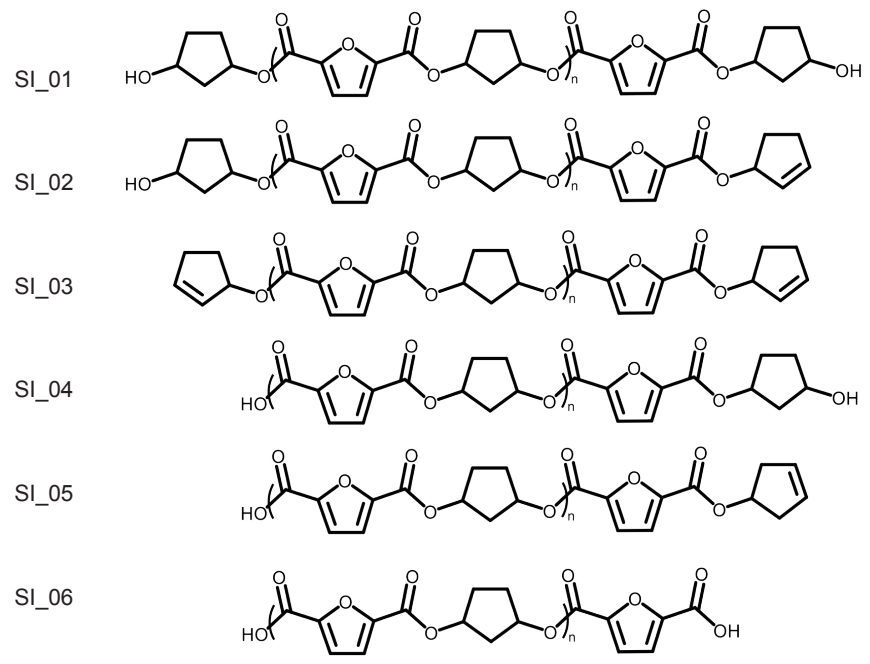

Figure S2 - 9. Expected linear distributions of poly(1,3-CP-F): cyclic (not shown), linear diol (01), dehydrated $(02,03)$, loss of CPol and dehydrated $(04,05,06)$. 


\section{MALDI-ToF-MS - CPene-CPene cross-coupling reactions}

One expected side reaction is the reaction between two cyclopentene groups, which are generated by the thermal dehydration of pendant cyclopentanol groups. These double bonds can undergo (thermal) radical addition reactions, forming a Cp-Cp bond (Figure S2-10). The expected structures are diol terminated (07), and dehydrated $(08,09)$. Further degradation can lead to loss of CPol group (10), combined with dehydration (11), eventually leading to di-acid terminated chains (12).

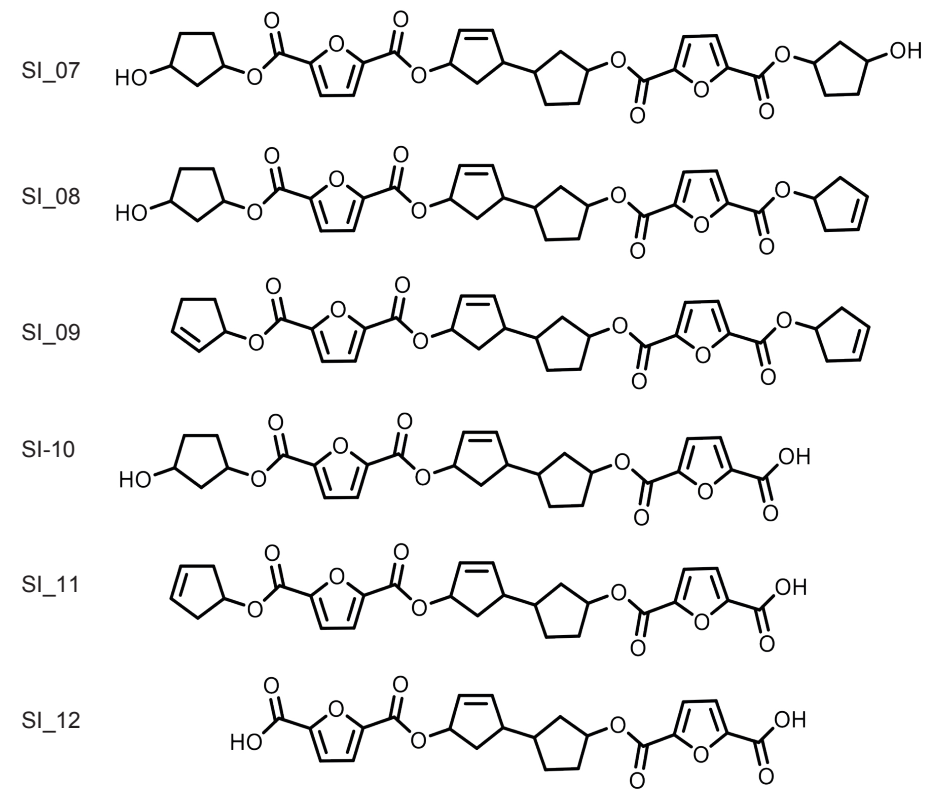

Figure S2 - 10. Expected linear distributions after CPene-CPene cross-coupling reaction. 


\section{Obtained MALDI-ToF-MS spectra}

In the following MALDI-ToF-MS spectra, and the text, the distributions are numbered in accordance to the structures in Figure S2-9 and Figure S2-10, e.g. linear diol-terminated chains SI_01, is denoted as 1 . The major ticks are aligned to the $M 0$ of the repeat unit CP-F (m/z 222.1), ranging from $n=2$ to $n=6$ of the linear diol-terminated chains 1 .

\section{Obtained MALDI-ToF-MS spectra $20 \%$ cis}

MALDI-ToF-MS spectra obtained for poly(CP-F) with $20 \%$ cis, after thermal stability experiments at $180{ }^{\circ} \mathrm{C}, 200{ }^{\circ} \mathrm{C}$ and $220{ }^{\circ} \mathrm{C}$. For the sample at $180{ }^{\circ} \mathrm{C}$ two distributions are found equally present: 1 linear diol terminated chains 1 , and linear di-acid terminated chains 6 . The third distribution is linear ene-acid terminated chains 5 . At $200{ }^{\circ} \mathrm{C}$ mostly linear diol terminated chains 1 are present, together with dehydrated linear chain 2, and linear di-acid terminated chain 6. At $220{ }^{\circ} \mathrm{C}$, linear diol terminated chains 1 disappears, with the appearance of dehydrated linear chains 2, and 3. Linear di-acid terminated chain 6 is almost gone, and $\mathrm{Cp}-\mathrm{Cp}$ addition chains 7, and the dehydration 8 and 9 are appearing. Please note that $\mathbf{6}$ and $\mathbf{8}$ are close, but have a mass-difference of 4 dalton.

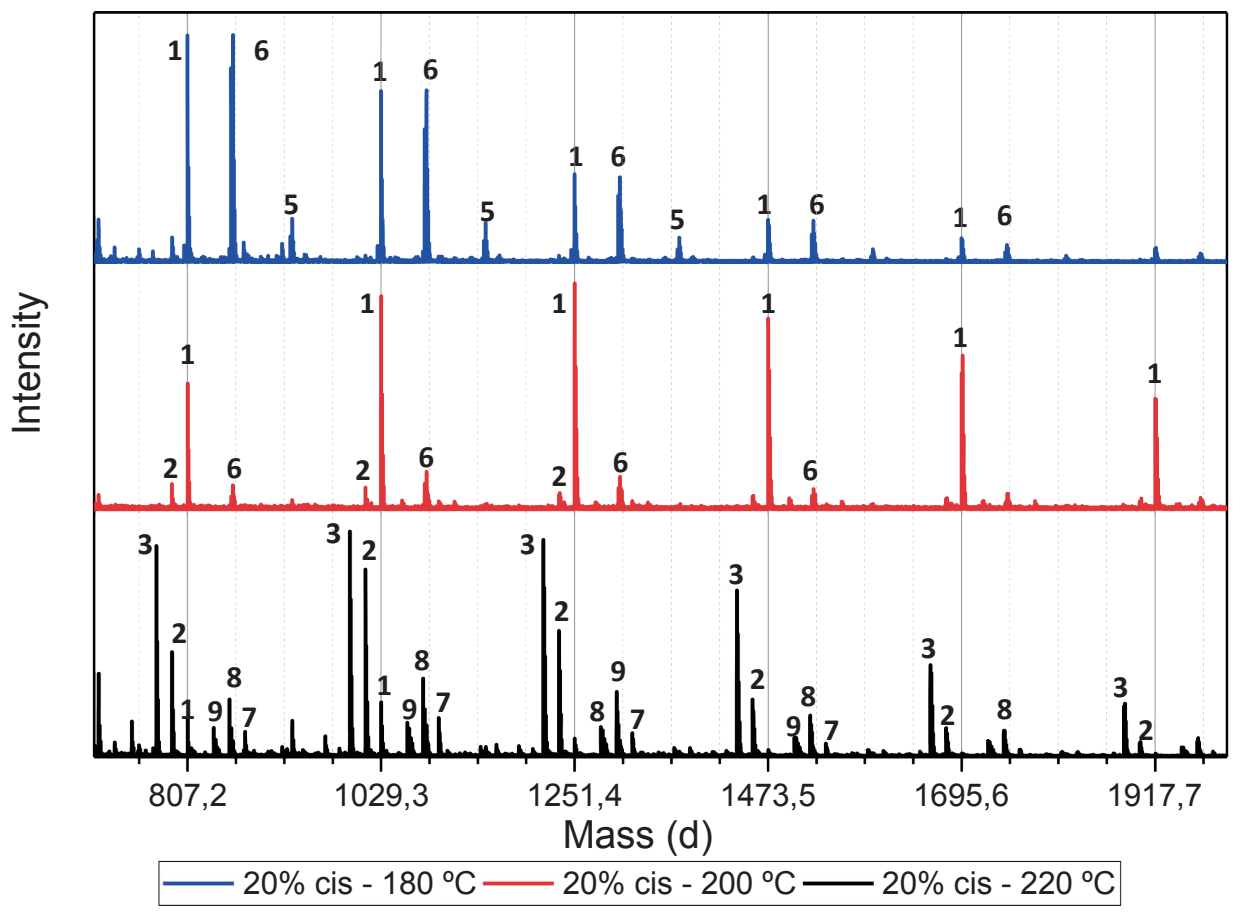

Figure S2 - 11. Obtained MALDI-ToF-MS spectra for poly(1,3-CP-F) with $20 \%$ cis, after thermal stability experiments at $180^{\circ} \mathrm{C}$ (blue, top), $200^{\circ} \mathrm{C}$ (red, middle), and $220^{\circ} \mathrm{C}$ (black, bottom). 
MALDI-ToF-MS spectra obtained for poly (CP-F) with $30 \%$ cis, after thermal stability experiments at $180{ }^{\circ} \mathrm{C}, 200{ }^{\circ} \mathrm{C}$ and $220{ }^{\circ} \mathrm{C}$. For the sample at $180{ }^{\circ} \mathrm{C}$ two main distributions are found: linear diol terminated chains 1 , and linear di-acid terminated chains 6. The third distribution is linear ene-acid terminated chains 5 . At $200{ }^{\circ} \mathrm{C}$ the linear di-acid terminated chain $\mathbf{6}$ is most abundant, together with linear diol terminated chains $\mathbf{1}$, and dehydrated linear chain $\mathbf{2}$. The fourth distribution is linear ene-acid terminated chains 5. At $220^{\circ} \mathrm{C}$, linear diol terminated chains 1 disappears, with the appearance of dehydrated linear chains $\mathbf{2}$, and $\mathbf{3}$. Linear di-acid terminated chain $\mathbf{6}$ is almost gone, but $\mathrm{Cp}-\mathrm{Cp}$ addition chains $\mathbf{7}$, and the dehydration $\mathbf{8}$ and $\mathbf{9}$ are appearing.

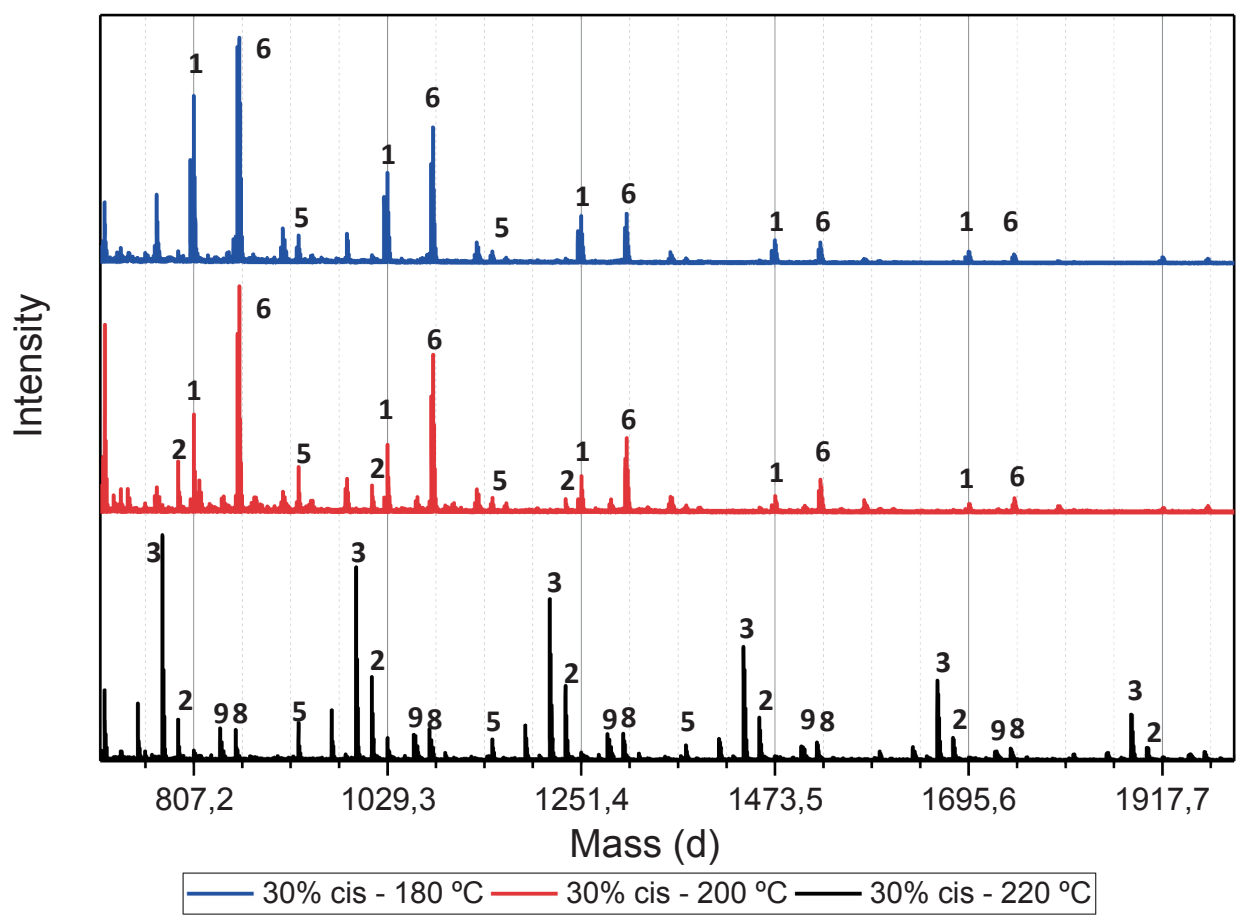

Figure S2 - 12. Obtained MALDI-ToF-MS spectra for poly(1,3-CP-F) with $30 \%$ cis, after thermal stability experiments at $180^{\circ} \mathrm{C}$ (blue, top), $200^{\circ} \mathrm{C}$ (red, middle), and $220^{\circ} \mathrm{C}$ (black, bottom). 
Obtained MALDI-ToF-MS spectra $40 \%$ cis

MALDI-ToF-MS spectra obtained for poly(CP-F) with $40 \%$ cis, after thermal stability experiments at $180{ }^{\circ} \mathrm{C}, 200{ }^{\circ} \mathrm{C}$ and $220{ }^{\circ} \mathrm{C}$. For the sample at $180{ }^{\circ} \mathrm{C}$ two distributions are found: linear diol terminated chains 1, and linear di-acid terminated chains 6 . At 200 ${ }^{\circ} \mathrm{C}$ the linear di-acid terminated chain 6 is most abundant, together with linear diol terminated chains 1, and dehydrated linear chain 2. The fourth distribution is linear eneacid terminated chains 5 . At $220{ }^{\circ} \mathrm{C}$, linear dehydrated chains 3 are most abundant. Dehydrated Cp-Cp adducts 8 and 9 have appeared alongside linear ene-acid terminated chains 5.

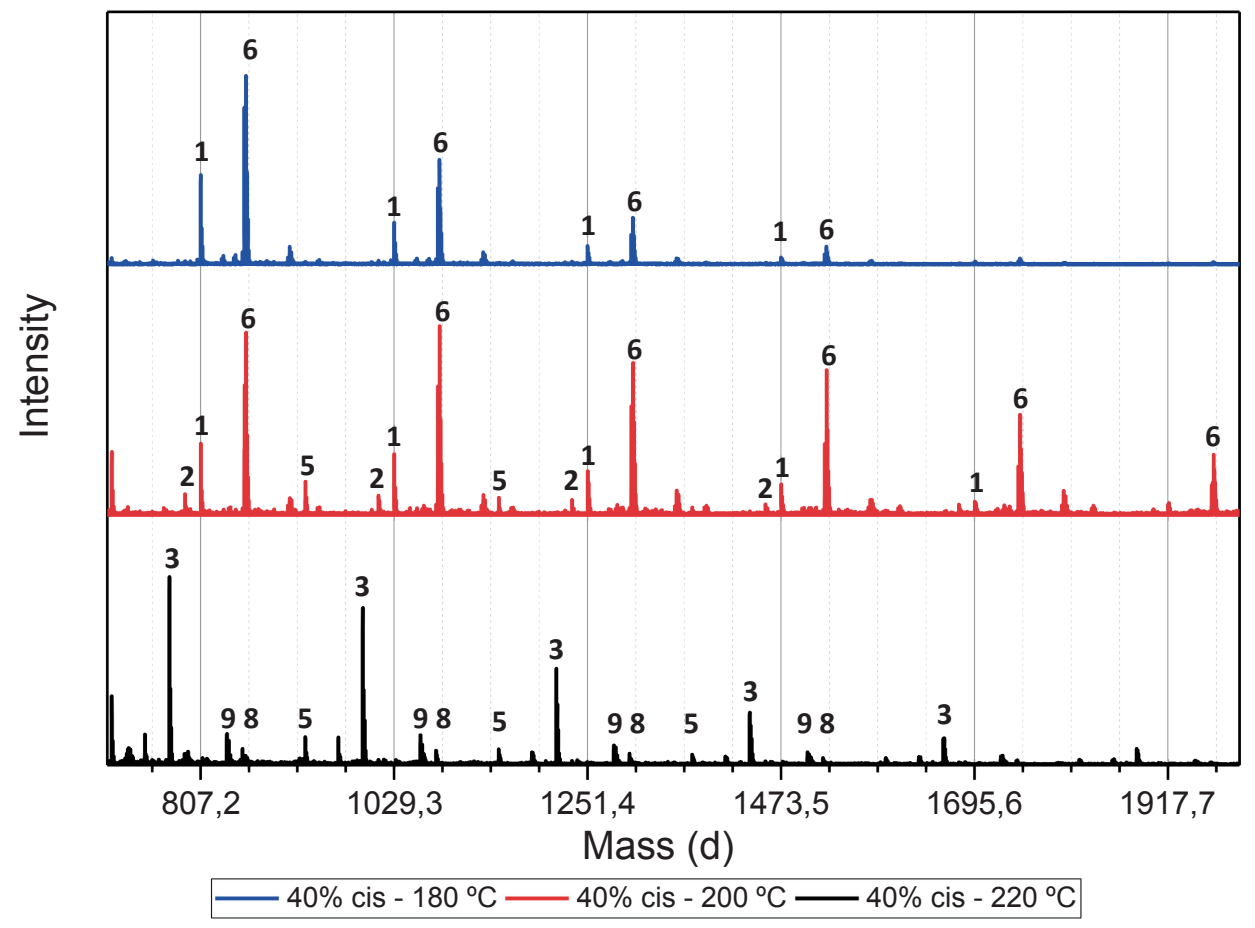

Figure S2 - 13. Obtained MALDI-ToF-MS spectra for poly(1,3-CP-F) with $40 \%$ cis, after thermal stability experiments at $180^{\circ} \mathrm{C}$ (blue, top), $200^{\circ} \mathrm{C}$ (red, middle), and $220^{\circ} \mathrm{C}$ (black, bottom). 


\section{Chapter 3 1,3-cyclopentanediol as comonomer in poly(ethylene furanoate) (PEF)}

This chapter is submitted for publication as:

Synthesis, thermal behavior and preliminary oxygen barrier properties of copolymers of 2,5-furandicarboxylic acid, 1,3-cyclopentanediol and ethylene glycol.

G.J. Noordzija,b, S. Rastogia ${ }^{a}$ D. Adons ${ }^{c}$, R. Peeters ${ }^{c}$, C.H.R.M. Wilsens ${ }^{a}$

a) Aachen-Maastricht Institute of Biobased Materials (AMIBM), Faculty of Science and Engineering, Maastricht University, Brightlands Chemelot Campus, 6167 RD Geleen, The Netherlands.

b) Chemelot InSciTe, Urmonderbaan 20F, NL-6167 RD Geleen, The Netherlands.

c) Hasselt University, IMO-IMOMEC, Packaging Technology Center, Wetenschapspark 27, 3590 Diepenbeek, Belgium

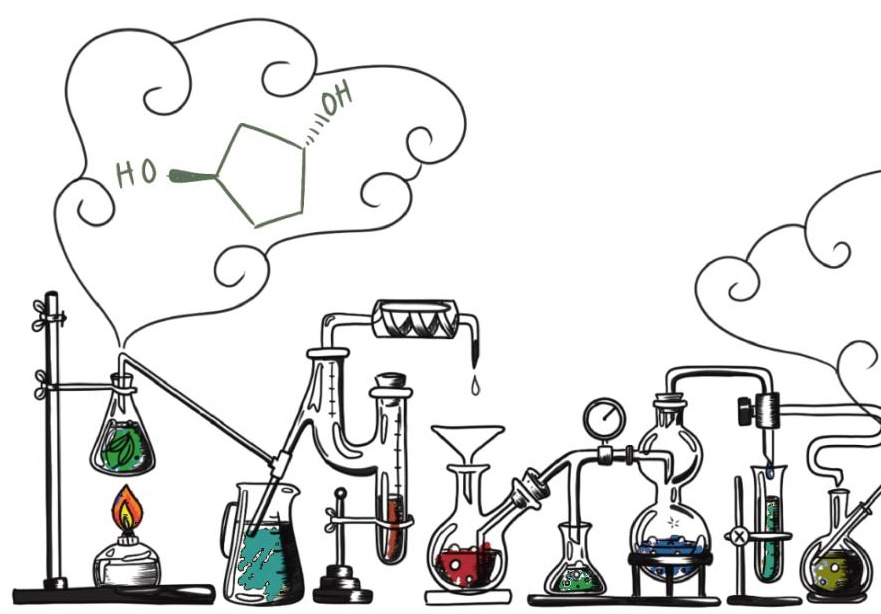




\section{Abstract}

In this work we report on the synthesis of a series of polymers containing the potentially renewable 1,3-cyclopentanediol (CPdiol) as comonomer in poly(ethylene furanoate) (PEF). Copolymers with molecular weights of more than $10 \mathrm{~kg} / \mathrm{mol}$ and up to $43 \%$ CPdiol can readily be synthesized via standard melt-polycondensation performed at $220{ }^{\circ} \mathrm{C}$, as is confirmed via GPC and ${ }^{1} \mathrm{H}-\mathrm{NMR}$ analysis. The introduction of CPdiol in the PEF backbone suppresses the glass transition temperature and limits the crystallization of PEF: copolymers containing 9-43\% CPdiol are fully amorphous and do not crystallize within the evaluated experimental conditions. A further increase in CPdiol content to $82 \%$ results in the formation of thermally unstable semicrystalline copolyesters that exhibit a melting temperature of $255^{\circ} \mathrm{C}$ and degrade upon melting. With respect to the thermal stability, thermogravimetric analysis studies performed at $240{ }^{\circ} \mathrm{C}$ confirm that increasing CPdiol content decreases the thermal stability of the materials, thereby limiting high-temperature applications and melt-processing temperatures. Furthermore, preliminary gas barrier experiments on amorphous films of PEF and the copolyesters with 5 and $24 \%$ CPdiol demonstrate that the oxygen transport rate (OTR) decreases at least fivefold in the presence of $24 \%$ CPdiol. In conclusion, we identify the 1,3-cyclopentanediol as a poor renewable alternative to cyclic diols such as 1,4-cyclohexanedimethanol for copolymerization in polycondensates, based on its limited thermal stability combined with the decrease in both oxygen barrier properties and $T_{\mathrm{g}}$, when introduced as comonomer in PEF.

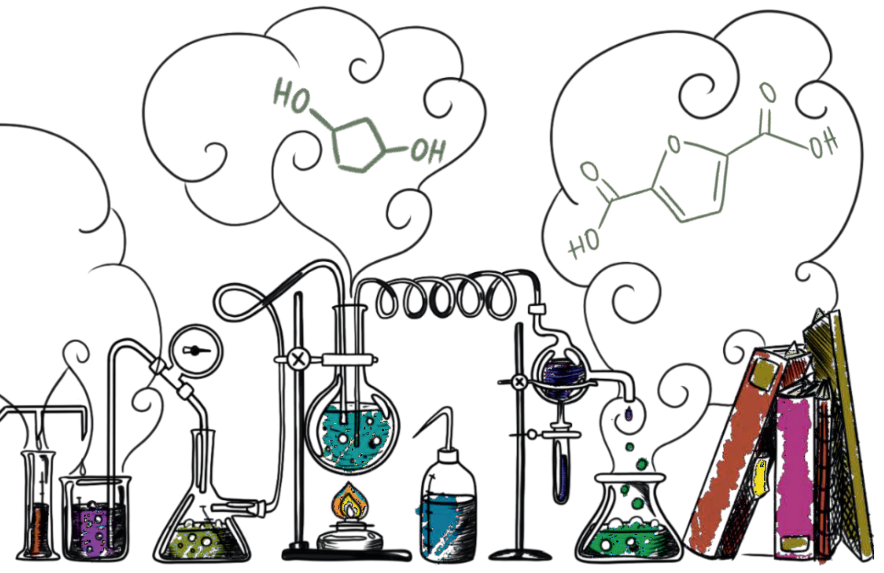




\section{Introduction}

Poly(ethylene furanoate) (PEF), based on 2,5-furandicarboxylic acid (FDCA), is a wellknown biobased replacement for non-renewable poly(ethylene terephthalate) (PET), with commercial ventures currently being undertaken by Avantium in the Netherlands. ${ }^{1}$ PEF reportedly displays enhanced gas barrier properties compared to PET for both $\mathrm{O}_{2}$ and $\mathrm{CO}_{2}$, making it a viable material for packing e.g. carbonated soft drinks. In addition, PEF exhibits a higher glass transition temperature $\left(T_{g}\right)$ than PET $\left(87 \mathrm{vs} .75^{\circ} \mathrm{C}\right)$, which enables the material to be used at slightly higher temperatures in production processes, foodfilling processes, and cleaning processes. In addition, the barrier properties towards $\mathrm{O}_{2}$, $\mathrm{CO}_{2}$, and $\mathrm{H}_{2} \mathrm{O}$ for PEF as compared to PET have been studied in detail by Burgess, Koros, and others, on both amorphous ${ }^{2-6}$ and semi-crystalline PEF. ${ }^{7}$ Although PEF exhibits a higher free volume compared to PET, PEF exhibits a lower chain mobility caused by the limited ability of the furan rings to rotate, a process that is hindered by the electronegative oxygen heteroatom. The resulting decrease in segmental mobility limits diffusion of gas molecules, and has been identified as the main reason for the improved barrier properties of PEF over PET. 2,8

A common approach to influence the chain mobility, thermal, and mechanical material properties in polycondensates is through copolymerization with different diol monomers. ${ }^{9-12}$. In particular, the copolymerization of PET with rigid cyclic diols such as 1,4-cyclohexanedimethanol $(\mathrm{CHdmol})^{13-15}, \quad$ 1,1,3,3-tetramethyl-2,4-cyclobutanediol (CBdiol) $)^{16}$, isohexides ${ }^{14,15,17}$, Manx-OH ${ }^{18,19}, \mathrm{Glux} \mathrm{OH}^{18}$, and Thx-diol ${ }^{20}$ has been reported and is generally accompanied by an increase in $T_{\mathrm{g}}$. However, the introduction of such rigid monomers in the polymer backbone generally disrupts the crystallization of the main polymers, and hence yields amorphous materials or polymers with decreased crystallinity. Similarly, FDCA based (co)polyesters based on a variety of diols have been studied $^{21}$ and reviewed ${ }^{22}$ for their thermal properties. These studies show that the polymerization with rigid cyclic diols such as $\mathrm{CBdiol}^{11,12,23}, \mathrm{CHdmol}^{10,12,24-27}$, and isohexides ${ }^{27}$ mostly increased the $T_{\mathrm{g}}$ of PEF from $87^{\circ} \mathrm{C}$ to values surpassing $100{ }^{\circ} \mathrm{C}$. Additionally, Wang, Zhu, and others reported on the change in gas barrier properties with copolymerization of various cyclic diols with PEF (Table 3-1).

To be able to compare the measured change in gas barrier properties of these copolyesters by different research groups, the Barrier Improvement Factor (BIF) is used. BIF ensures that the results from comparative studies, even though these studies may have been performed under different conditions, can be normalized and compared to each other. For example, several studies reported an increase in $\mathrm{CO}_{2}$-barrier properties of 11-19x for PEF as compared to bottle-grade PET. ${ }^{6,11}$ Furthermore, as is summarized in Table 3-1, the copolymerization of cyclic diols in PEF is generally accompanied by a decrease in gas barrier properties. Nevertheless, despite the trade-off between the enhancement in $T_{\mathrm{g}}$ and the decrease in barrier properties, the gas barrier properties of these copolymers is still higher compared to bottle-grade PET. 
Table 3-1. Literature overview of PEF copolyesters with various cyclic diols, listing the change in $T_{\mathrm{g}}$ and the Barrier Improvement Factor (BIF) towards $\mathrm{CO}_{2}$ and $\mathrm{O}_{2}$ as compared to PET. Letter-coding is taken from original publication, the number indicates the percentage of cyclic diol monomer in the polymer. ${ }^{a}$ ) cis/trans ratio $32 / 68$. ${ }^{\mathrm{B}}$ ) cis/trans ratio 90/10. ${ }^{C}$ ) cis/trans ratio $45 / 55$. D) cis/trans ratio $2 / 98$.

\begin{tabular}{|c|c|c|c|c|c|c|}
\hline Polymer & Comonomer 1 & Comonomer 2 & $T_{\mathrm{g}}\left({ }^{\circ} \mathrm{C}\right)$ & $\mathrm{BIF} \mathrm{CO}_{2}$ & $\mathrm{BIF} \mathrm{O}_{2}$ & Reference \\
\hline PET & & & 70 & 1 & 1 & \\
\hline PEF & & & 87 & $11-19$ & $5.5-11$ & $3,6,11$ \\
\hline PEF & CBdiol-10 & & 90.9 & 6.8 & 4.6 & 11 \\
\hline PEF & CBdiol-23 & & 94.3 & 2.2 & 2.1 & 11 \\
\hline$P E F$ & $\mathrm{CHdmol}^{\mathrm{a}}-32$ & & 83.8 & 10.8 & 4.3 & 10 \\
\hline$P E F$ & $\mathrm{CHdmol}^{\mathrm{a}}-76$ & & 81 & 4.8 & $1.3-3.5$ & 10,24 \\
\hline$P E F$ & $\mathrm{CHdmol}^{\mathrm{b}}-75$ & & 71.5 & 2.5 & 2.3 & 25 \\
\hline$P E F$ & $\mathrm{CHdmol}^{\mathrm{c}}-75$ & & 78.9 & 5 & 4.3 & 25 \\
\hline PEF & $\mathrm{CHdmol}^{\mathrm{d}}-75$ & & 89.2 & 6.8 & 6.7 & 25 \\
\hline PEF & $\mathrm{CHdmol}^{\mathrm{a}}-66$ & CBdiol-15 & 104.4 & - & - & 12 \\
\hline$P E F$ & $\mathrm{CHdmol}^{\mathrm{a}}-25$ & CBdiol-53 & 119.6 & - & - & 12 \\
\hline PEF & Isosorbide-10 & CHdmol-40 & 90.3 & - & - & 27 \\
\hline PEF & Isosorbide-30 & CHdmol-20 & 119.0 & - & - & 27 \\
\hline
\end{tabular}

Recently, we reported on polyesters containing 1,3-cyclopentanediol (CPdiol) ${ }^{28}$, a cycloaliphatic monomer which can be obtained from furfuryl alcohol. ${ }^{29}$ Though CPdiol suffers from limited thermal stability, it can readily be used in melt-polycondensations at temperatures around or below $200{ }^{\circ} \mathrm{C}$. Under such reaction conditions, polyesters based on CPdiol and FDCA, terephthalic acid, adipic acid, or sebacic acid were reported with molecular weights $\left(M_{\mathrm{w}}\right)$ between 10 to $92 \mathrm{~kg} / \mathrm{mol}$ (according to GPC analysis referenced against PMMA standards). Interestingly, polyesters based on CPdiol proved comparable in terms of thermal behavior to polyesters based on CHdmol. Therefore, to identify whether CPdiol can be a renewable alternative to the commonly used cyclic comonomers in PEF, we report on the copolymerization of 2,5-furandicarboxylic acid, ethylene glycol and 1,3-cyclopentanediol, the thermal behavior of the resulting polymers and preliminary gas barrier properties. 


\section{Materials and Methods}

\section{Materials}

1,3-Cyclopentanediol (15/85 cis/trans), Irganox 1010 and Ti(IV)isopropoxide were obtained from Sigma Aldrich. Dimethyl-2,5-furandicarboxylate was obtained from ABCR. Ethylene glycol was obtained from TCI. Standard laboratory solvents were obtained from Biosolve. Deuterated solvents were obtained from Buchem BV (Netherlands). Anhydrous toluene was obtained after passing through a solvent purifier system. The purchased compounds were used directly without further purification, unless otherwise specified.

\section{Characterization methods}

${ }^{1} \mathrm{H}-\mathrm{NMR}$ and ${ }^{13} \mathrm{C}-\mathrm{NMR}$ spectra were recorded with a Bruker Ultrashield 300 spectrometer $(300 \mathrm{MHz}$ magnetic field). NMR-samples were prepared by dissolving ca. $10 \mathrm{mg}$ of sample in $0.5 \mathrm{~mL}$ deuterated solvent, including dimethyl sulfoxide (DMSO- $\left.d_{6}\right)$, deuterated chloroform $\left(\mathrm{CDCl}_{3}\right)$, and deuterated trifluoroacetic acid ( $d$-TFA). All spectra were referenced against tetramethylsilane (TMS), or residual solvent peak from the deuterated solvent. Molecular weight $\left(M_{\mathrm{n}}, M_{\mathrm{w}}\right)$ and dispersity $(\boxplus)$ of the polymers were calculated after gel permeation chromatography (GPC). $\mathrm{CHCl}_{3}-\mathrm{GPC}$ was measured on a Prominence-I LC-2030 equipped with a Shodex GPC KF-805L column. Analytical grade $\mathrm{CHCl}_{3}$ was used as mobile phase at $40{ }^{\circ} \mathrm{C}$, with a flowrate of $1 \mathrm{~mL} / \mathrm{min}$. GPC samples were prepared by dissolving ca. 5 $\mathrm{mg}$ of polymer in $1.5 \mathrm{~mL}$ of solvent overnight under constant shaking, the samples were filtered over a 0.2 $\mu \mathrm{m}$ PTFE syringe filter prior to injection. Thermal stability of compounds and screening of polymerization conditions were performed via thermogravimetric analysis (TGA) using a TA Instruments Q500. Experiments were performed under a nitrogen atmosphere with a heating rate of 1 or $10{ }^{\circ} \mathrm{C} / \mathrm{min}$. Thermal transition temperatures of the polymers were analyzed via differential scanning calorimetry (DSC) using a TA Instruments DSC Q2000. Typically, two heating and cooling runs were performed at a rate of $10{ }^{\circ} \mathrm{C} / \mathrm{min}$, the first heating was used to erase any thermal history in the samples. The glass-transition temperature $\left(T_{\mathrm{g}}\right)$ was obtained from the first heating of quenched polymer samples, peak melt-temperature $\left(T_{\mathrm{m}}\right)$ was obtained from the second heating run. DSC samples were prepared by loading 3-5 mg oven-dried samples in Tzero Hermetic Aluminum pans. Polarized optical microscopy (POM) imaging was recorded on an Olympus BX53DP 26, equipped with a Linkam AFSX53 Hotstage. POM was used to determine melttemperatures of samples which could not be measured via DSC (e.g. because of degradation at melt). POM samples were loaded on a microscopy slide and heated at a rate of $10{ }^{\circ} \mathrm{C} / \mathrm{min}$, where the melttemperature or range was determined by visual aid of the samples becoming anisotropic in nature.

\section{Gas barrier measurements}

The permeation of oxygen is measured at the Packaging Technology Center / imo-imomec according ASTM (American Society for Testing and Materials) F-1927 using an OX-TRAN® 2/22 (AMETEK MOCON Inc), which applies the equal pressure method using a coulometric detector. Amorphous films of copolymers with a diameter of $\sim 3 \mathrm{~cm}$, and a thickness between 600-800 $\mu \mathrm{m}$ have been prepared via vacuum-melt pressing in between kapton sheets between 180 and $210{ }^{\circ} \mathrm{C}$. The films were obtained as amorphous by quenching the films by rapidly cooling from the melt $\left(180-210^{\circ} \mathrm{C}\right)$ to room temperature in air. As expected from the slow crystallization kinetics of PEF and the synthesized copolymers, this quenching route yielded solely amorphous transparent films.

A standard preparation of ' $50 \mathrm{~cm}^{2}$ '-samples was not possible due the small applicable sample area. A reduction of the test surface is done by using aluminum holder sheets (masks). The test sample is placed in between 2 aluminium stickers with an opening of $5 \mathrm{~cm}^{2}$. The border between the holder sheet and the polymer films were closed with 2-component glue to avoid leakages (on both sides of each test sample) (SI). This results in an average sample test area of $3.66 \mathrm{~cm}^{2}$. The test samples were stored in a controlled 
environment with a constant $50 \%$ relative humidity $(\mathrm{RH})$ at $23{ }^{\circ} \mathrm{C}$ prior to use. The prepared samples are mounted into the test cell, between two test compartments. The sweep gas or downstream side is purged with forming gas, whereas oxygen is introduced in the test gas or upstream side. The coulometric detector determines the absolute amount of oxygen, which has permeated into the sweep gas. Due to the presence of oxygen in air, the leakage into the system is determined by performing an individual zero (IZ) measurement by allowing the sweep gas to purge both cell compartments. This IZ is subtracted from the permeated amount. The measurements were performed at $23^{\circ} \mathrm{C}$ and $50 \% \mathrm{RH}$. The OTR is expressed in $\left[\mathrm{cc} / \mathrm{m}^{2} \cdot\right.$ day $\left.\cdot \mathrm{atm}\right]$. The test orientation is free.

\section{Synthesis methods}

\section{Melt-polycondensation}

Copolyesters based on dimethyl 2,5-furandicarboxylate (dmFDCA), 1,2-ethylene glycol (EG), and varying amounts 1,3-cylopentanediol (CPdiol) have been prepared via a typical two-step melt polycondensation reaction (Scheme 3-1). A ratio of 1 : 1.6-2.2 of dmFDCA to diol was used, with varying amounts of CPdiol to EG depending on the desired CPdiol content (Table 3-2). Ti(IV)isopropoxide $(0.5 \mathrm{~mol} \%$ to dmFDCA in $1 \mathrm{~mL}$ anhydrous toluene) was added as transesterification catalyst, Irganox 1010 (0.25 mol\% to dmFDCA) was added as antioxidant. The oligomerization temperature was set at $180{ }^{\circ} \mathrm{C}$ for $4-6$ hours under a light $\mathrm{N}_{2}$ flow to distill of the methanol as condensate, captured in a condensing flask equipped after a short vigreux column. The melt-polycondensation was performed between 195 and $230{ }^{\circ} \mathrm{C}$ for $6-8$ hours, while reducing the pressure to values below $1 \mathrm{~Pa}$. The polymers were named according to their CPdiol contents, for example, ECP9 contains 9 mol\% CPdiol and 91 mol\% EG.

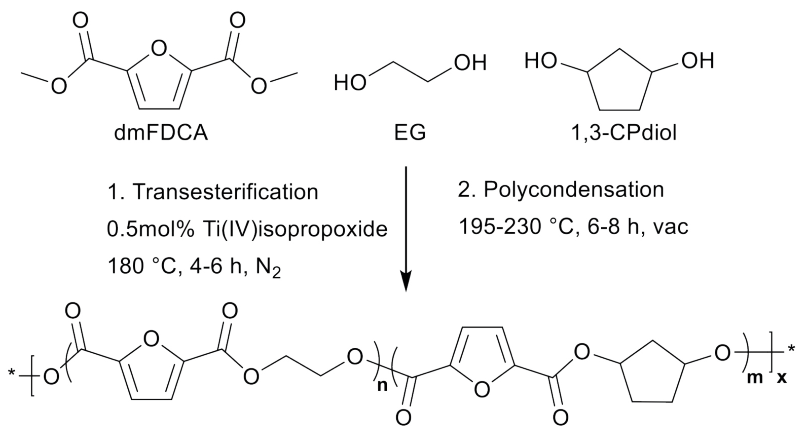

Scheme 3-1.Schematic overview of the copolymerization of dmFDCA, EG, and 1,3-CPdiol.

Table 3-2. Overview of monomer feed ratios and polymerization conditions for the preparation of copolymers of PEF with varying amounts of CPdiol.

\begin{tabular}{|c|c|c|c|c|c|}
\hline Sample & $\begin{array}{l}\text { dmFDCA } \\
(\mathrm{g} / \mathrm{mmol})\end{array}$ & $\begin{array}{l}\text { CPdiol } \\
\text { (g/ mmol) }\end{array}$ & $\begin{array}{c}\text { EG } \\
\text { (g/ } / \mathrm{mmol})\end{array}$ & $\begin{array}{c}\text { Mol ratio } \\
\text { dmFDCA/CPdiol/EG }\end{array}$ & $\begin{array}{l}\text { Polycondensation } \\
\text { temperature }\end{array}$ \\
\hline PEF & $2.5 / 13.3$ & 0 & $1.81 / 29.2$ & $1 / 2.2$ & $230^{\circ} \mathrm{C}$ \\
\hline ECP5 & $2.5 / 13.3$ & $0.095 / 0.93$ & $1.76 / 28.3$ & $1 / 0.07 / 2.13$ & $220^{\circ} \mathrm{C}$ \\
\hline$E C P 9$ & $2.5 / 13.3$ & $0.163 / 1.59$ & $1.63 / 26.3$ & $1 / 0.12 / 1.98$ & $195^{\circ} \mathrm{C}$ \\
\hline$E C P 24$ & $2.5 / 13.3$ & $0.41 / 3.99$ & $1.40 / 22.6$ & $1 / 0.3 / 1.7$ & $195^{\circ} \mathrm{C}$ \\
\hline ECP43 & $2.5 / 13.3$ & $0.75 / 7.31$ & $1.20 / 19.3$ & $1 / 0.55 / 0.45$ & $195^{\circ} \mathrm{C}$ \\
\hline ECP82 & $2.5 / 13.3$ & $1.09 / 10.63$ & $0.66 / 10.6$ & $1 / 0.8 / 0.8$ & $220^{\circ} \mathrm{C}$ \\
\hline
\end{tabular}




\section{Results and Discussion}

Copolyesters based on dmFDCA, EG and CPdiol were synthesized via the two-step meltpolycondensation protocol described in the experimental section. An excess of diol was used (Table 3-2) to compensate for the evaporation of diols during the oligomerization step, resulting from the low melting and boiling temperatures of both EG and CPdiol. In general, given the higher rate of evaporation for EG compared to CPdiol, the ratio of dmFDCA to diol was varied 1:2.2 for the polymer containing no CPdiol to 1:1.6 for the polymer containing $75 \% \mathrm{CPdiol}$. The polymers were successfully isolated from the melt and subjected to both NMR and GPC analysis. Figure 3-1 shows the ${ }^{1} \mathrm{H}-\mathrm{NMR}$ spectra and denotation of protons of the polymers in the region between 0 and $8 \mathrm{ppm}$. 2D-NMR analysis was performed to assign all peaks, and the results are provided in the SI. In general, the presence of CPdiol groups can be identified by the signals $\mathbf{5}-\mathbf{8}$, where signals $\mathbf{5}$ originate from CPdiol in the backbone, and $\mathbf{8}$ from CPdiol as end-group. Note, signal $\mathbf{5}$ is split into two peaks resulting from the cis/trans ratio of the CPdiol, where signals at $5.65 \mathrm{ppm}$ and $5.55 \mathrm{ppm}$ originate from the trans-isomer and cis-isomers, respectively. The presence of 2,5-FDCA and EG is confirmed by signal 1 and signals 2 and 3, respectively. The ratio of EG to CPdiol present in the final polymers (Table 3-3) was determined by taking the ratio between signal $\mathbf{2}(\mathrm{EG}, 4.7 \mathrm{ppm})$ and signal $\mathbf{5}$ (CP, 5.5 $\mathrm{ppm}$ ), and correcting for the number of protons (signal $2=4 \mathrm{H}$, signal $5=2 \mathrm{H}$ ). The values used to determine the EG to CPdiol ratio are provided in Table S1 (SI). Note, the copolyesters contain an unidentified peak at ${ }^{1} \mathrm{H} 1.43 \mathrm{ppm},{ }^{13} \mathrm{C} 30.1 \mathrm{ppm}$, however COSYNMR indicates that the proton responsible for this signal is unattached to the polymer, and is likely an impurity (SI).

Table 3-3. Overview of obtained copolyester properties. $T_{\mathrm{g}}$ and $T_{\mathrm{m}}$ values are obtained from the $1^{\text {st }}$ heating $\left(1^{\circ} \mathrm{C} / \mathrm{min}\right)$ of quenched polymer samples where any thermal history is erased. ${ }^{*}$ ) $T_{\mathrm{m}}$ obtained via POM equipped with a hotstage in the second heating of quenched samples $\left(10^{\circ} \mathrm{C} / \mathrm{min}\right)$.

\begin{tabular}{|c|c|c|c|c|c|c|c|c|}
\hline Sample & Polymer color & $\begin{array}{l}\text { CPdiol feed } \\
\text { (mol\%) }\end{array}$ & $\begin{array}{c}\text { CPdiol }{ }^{1} \mathrm{H}-\mathrm{NMR} \\
(\mathrm{mol} \%)\end{array}$ & $\begin{array}{c}M_{\mathrm{n}} \\
(\mathrm{g} / \mathrm{mol})\end{array}$ & $\underset{(\mathrm{g} / \mathrm{mol})}{M_{\mathrm{w}}}$ & $\bigoplus$ & $T_{\mathrm{g}}\left({ }^{\circ} \mathrm{C}\right)$ & $T_{\mathrm{m}}\left({ }^{\circ} \mathrm{C}\right)$ \\
\hline$E F$ & Light brown & $0 \%$ & $0 \%$ & $15.3 k$ & $44.1 \mathrm{k}$ & 2.9 & 77.4 & 217.6 \\
\hline ECP5 & Light brown & $7 \%$ & $5 \%$ & $15.7 \mathrm{k}$ & $29.8 k$ & 1.9 & 75.9 & 208.2 \\
\hline ECP9 & Light brown & $12 \%$ & $9 \%$ & $7.3 \mathrm{k}$ & $12.0 \mathrm{k}$ & 1.6 & 69.3 & - \\
\hline$E C P 24$ & Transparent brown & $30 \%$ & $24 \%$ & $11.0 \mathrm{k}$ & $18.9 \mathrm{k}$ & 1.7 & 76.5 & - \\
\hline ECP43 & Transparent yellow & $55 \%$ & $43 \%$ & $8.6 \mathrm{k}$ & $14.8 \mathrm{k}$ & 1.7 & 69.5 & - \\
\hline ECP82 & Off-white & $80 \%$ & $82 \%$ & $3.1 \mathrm{k}$ & $6.3 \mathrm{k}$ & 2.0 & 59.9 & $255^{*}$ \\
\hline
\end{tabular}




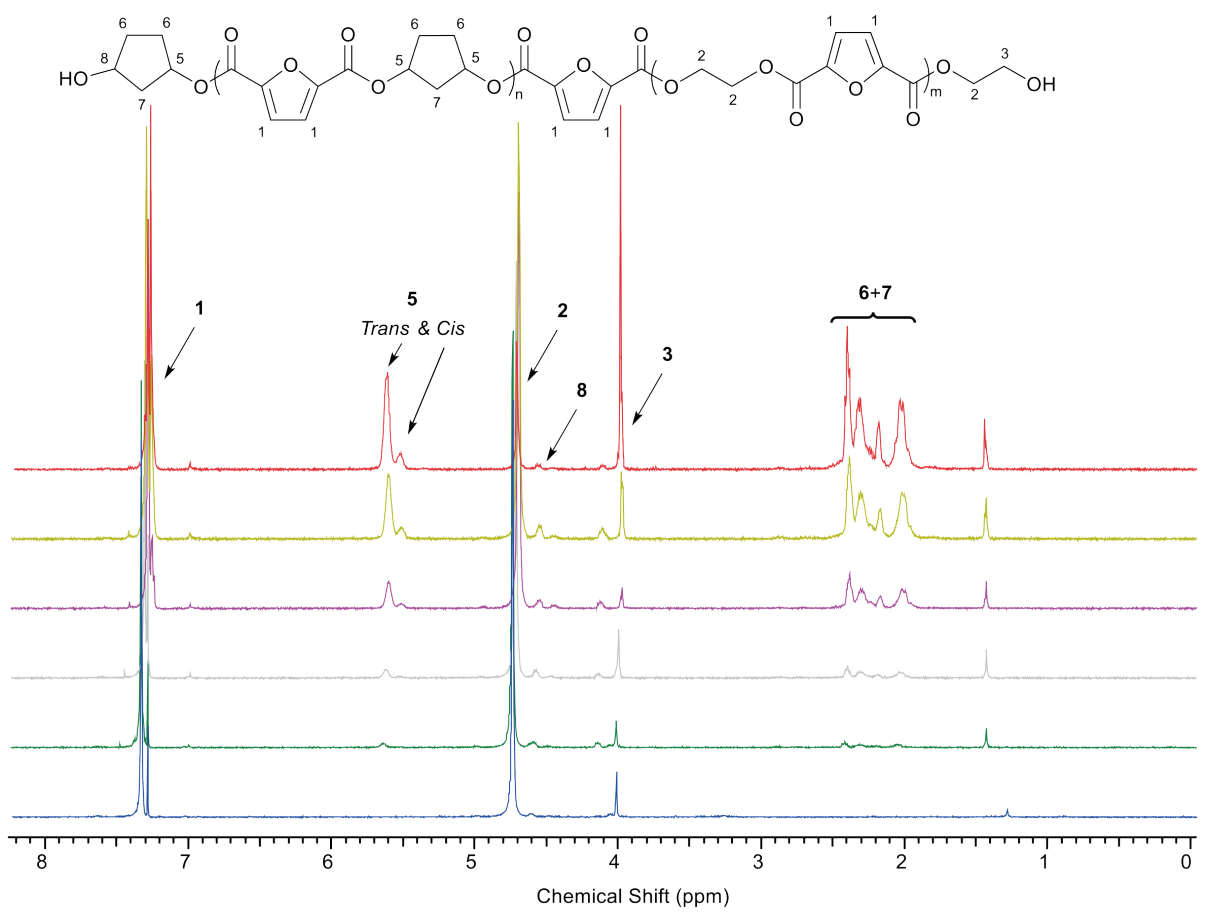

Figure 3-1. Overlay ${ }^{1} \mathrm{H}-\mathrm{NMR}$ of the prepared copolyesters, from bottom to top: PEF (blue), ECP5 (green), ECP9 (grey), ECP24 (purple), ECP43 (gold), and ECP82 (red).

Based on GPC analysis, we observe that the polymers display a dispersity ( $\boxminus$ ) between 1.6 and 2.9 (Table 3-3), close to the theoretical value of 2 for ideal transesterification reactions. Furthermore, both $M_{n}$ and $M_{\mathrm{w}}$ decrease gradually with increasing amount of CPdiol, suggesting that CPdiol exhibits a lower reactivity than EG. Nevertheless, the polymers containing $43 \%$ of CPdiol or less have a $M_{w}$ well above $10 \mathrm{~kg} / \mathrm{mol}$. The polymer containing $82 \%$ CPdiol, ECP82, crystallized during the melt-polycondensation as the $T_{\mathrm{m}}$ $\left(255^{\circ} \mathrm{C}\right)$ is substantially higher than the polycondensation temperature, which prevented efficient build-up of molecular weight. However, as we reported in previous work both CPdiol, and oligomers with CPdiol are unstable over prolonged heating above $220^{\circ} \mathrm{C}^{28}$, we did not pursue polymerization at temperatures beyond $220{ }^{\circ} \mathrm{C}$. Despite these unfavorable reaction conditions, polymer ECP82 was obtained with a $M_{\mathrm{w}}$ of $6 \mathrm{~kg} / \mathrm{mol}$.

Thermal characterization of the copolymers was performed using differential scanning calorimetry to identify the effect of CPdiol on both the glass transition temperature $\left(T_{\mathrm{g}}\right)$ and the melting $\left(T_{\mathrm{m}}\right)$ and crystallization behavior (Table 3-3). Prior to analysis the samples were quenched and subjected to a heating run at a rate of $10^{\circ} \mathrm{C}$ and $1{ }^{\circ} \mathrm{C} / \mathrm{min}$, shown in Figure 3-2 left and right, respectively. The heating rate of $1^{\circ} \mathrm{C} / \mathrm{min}$ was selected as the pure PEF did not display any notable crystallization during cooling or heating at a rate of $10^{\circ} \mathrm{C} / \mathrm{min}$. 
The addition of CPdiol as comonomer in PEF results in a slight suppression in glass transition temperature, as is highlighted by the blue dotted arrow in Figure 3-2, left. Though this suppression in $T_{\mathrm{g}}$ could originate from the decreasing molecular weights when incorporating higher amount of CPdiol, it is clear that CPdiol does not facilitate a $T_{\mathrm{g}}$ increasing effect as observed for other monomers such as $\mathrm{CHdiol}$ or $\mathrm{CHdmol} .{ }^{30}$ With respect to the crystallization behavior, PEF, ECP5, and ECP82 show cold-crystallization ( $T_{\mathrm{cc}}$ ) during heating beyond the $T_{\mathrm{g}}$ (while heating a rate of $1{ }^{\circ} \mathrm{C} / \mathrm{min}$, Figure 3-2, right). Furthermore, both PEF and ECP5 display distinct melting endotherms with peak values at 218 and $208{ }^{\circ} \mathrm{C}$, respectively. The decrease in $T_{\mathrm{m}}$ and increase in $T_{\mathrm{cc}}$ for ECP5 compared to PEF likely originates from the inability of CPdiol to fit in the PEF crystal structure, thereby disrupting the PEF crystallization process. When increasing the CPdiol content to 9 and up to $43 \%$ yields amorphous copolyesters, deduced by the absence of any notable cold-crystallization and melting transitions. These findings confirm that CPdiol does not co-crystallize with PEF. When increasing the CPdiol content further (polymer ECP82), again a cold-crystallization transition is observed, likely originating from crystallization of the poly(1,3-cyclopentane 2,5-furadicarboxylate) segments. Unfortunately, considering the limited thermal stability (Figure 3-3), DSC measurements could not be performed to determine the final $T_{\mathrm{m}}$ of the crystallites formed during coldcrystallization. Instead, the melting temperature of ECP82 was determined using polarization optical microscopy mounted with a linkam hotstage, where melting was observed at $255^{\circ} \mathrm{C}$, followed by immediate degradation.
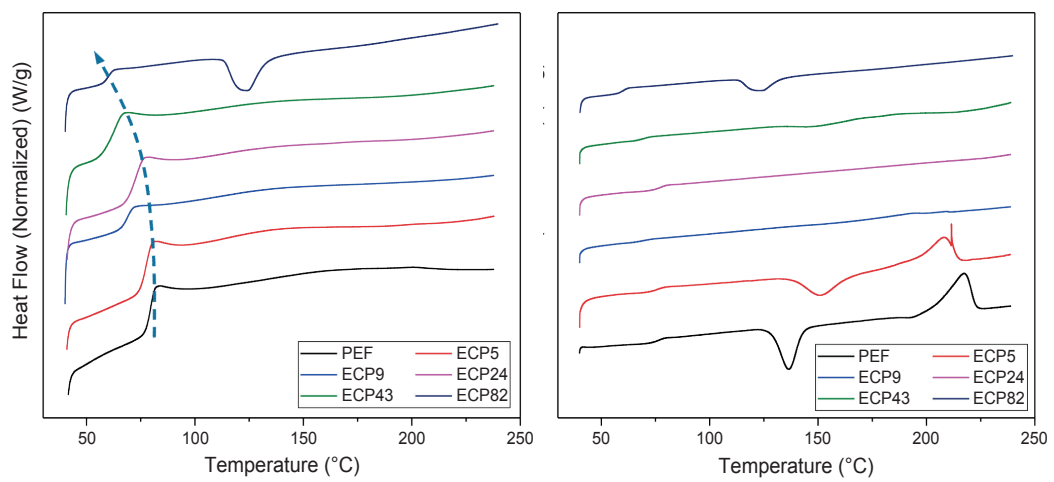

Figure 3-2. DSC measurements of the obtained copolyesters to show the effect on crystallinity upon addition of CPdiol. Shown is the $1^{\text {st }}$ DSC heating run of quenched polymer samples; Left: a heating rate of $10^{\circ} \mathrm{C} / \mathrm{min}$, showing that all samples except ECP82 do not cold-crystallize under quiescent conditions. It is visible how the addition of CPdiol appears to suppress the $T_{\mathrm{g}}$ (dashed blue line) Right: a heating rate of $1{ }^{\circ} \mathrm{C} / \mathrm{min}$ showing that PEF and ECP5 cold-crystallize, and that the addition of a small amount of CPdiol hampers the cold-crystallization rate, and leads to a reduced melting temperature of the obtained crystal domains.

Given the previously reported limited thermal stability of oligomers of 1,3-CPdiol and 2,5FDCA $^{28}$, the thermal degradation of the copolyesters was studied using TGA under isothermal conditions at $240^{\circ} \mathrm{C}$ for an hour (Figure 3-3, dotted black line). It is apparent how the copolyesters gradually decrease in weight over time upon reaching $240{ }^{\circ} \mathrm{C}$, furthermore the weight loss and discoloration of the polymers increases with increasing 
CPdiol content of the copolyesters (SI). To identify the molecular weight and its distribution after the isotherm at $240{ }^{\circ} \mathrm{C}$, the materials were dissolved in HFIP and subjected to GPC analysis (Table 3-4). Exposure of PEF to $240{ }^{\circ} \mathrm{C}$ slightly broadens the dispersity, but does not significantly affect the molecular weight of the material. In contrast, the $M_{\mathrm{n}}$ decreases significantly for polymers with increasing amounts of CPdiol, decreasing to values well below $1 \mathrm{~kg} / \mathrm{mol}$ for polymers containing $24 \%$ or more CPdiol. This observed instability reflects previous findings where it was shown that both CPdiol and its esters are thermally instable and are prone to undergo dehydration, followed by cross-coupling reactions resulting in branched and eventually heavily cross-linked polymer material. ${ }^{28}$ Indeed, similar degradation, branching and cross-linking seems to occur here, as the $M_{\mathrm{w}}$ of the polymers containing $9 \%$ CPdiol and higher increase significantly upon exposure to $240^{\circ} \mathrm{C}$, suggesting the occurrence of a branching or crosslinking reaction. Furthermore, the dispersity increases and black insoluble particles are formed for the copolymers with $24 \%$ and more CPdiol, after treatment at $240{ }^{\circ} \mathrm{C}$. Overall, these findings demonstrate that melt-processing times of (co)polyesters based on CPdiol should be minimized and preferably should not be conducted at temperatures of $240{ }^{\circ} \mathrm{C}$ and beyond.

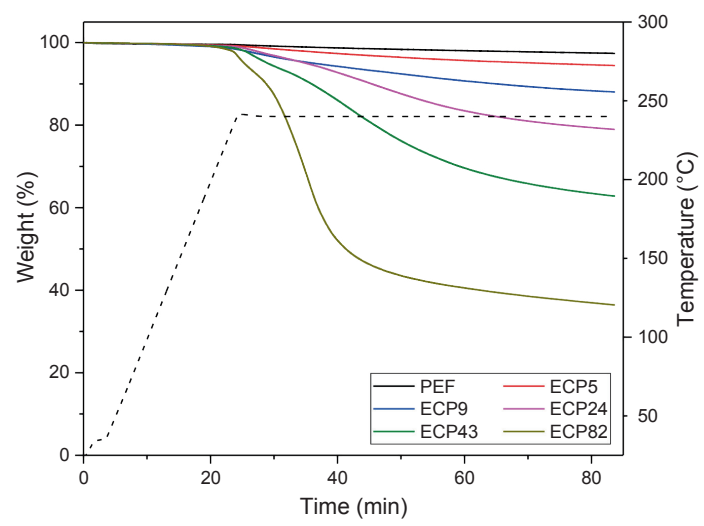

Figure 3-3. TGA measurements of the synthesized copolyesters showing thermal stability at $240{ }^{\circ} \mathrm{C}$. Heating profile (dotted black line): $10{ }^{\circ} \mathrm{C} / \mathrm{min}$ to $240^{\circ} \mathrm{C}, 1 \mathrm{~h}$ isothermal at $240{ }^{\circ} \mathrm{C}$. It is apparent that with an increase in CPdiol the weight loss at $240^{\circ} \mathrm{C}$ increases as compared to PEF (black).

Table 3-4. GPC measurements of the synthesized copolyesters before and after an hour of isothermal treatment at $240{ }^{\circ} \mathrm{C}$.

\begin{tabular}{|c|c|c|c|c|c|}
\hline Sample & $\begin{array}{c}\text { Weight } \\
\text { loss (\%) }\end{array}$ & $\begin{array}{c}M \mathrm{n} \text { before } \\
(\mathrm{g} / \mathrm{mol})\end{array}$ & $\begin{array}{c}M_{w} \text { before } \\
(\mathrm{g} / \mathrm{mol})\end{array}$ & $\begin{array}{c}M_{\mathrm{n}} \text { after } \\
(\mathrm{g} / \mathrm{mol})\end{array}$ & $\begin{array}{c}M_{\mathrm{w}} \text { after } \\
(\mathrm{g} / \mathrm{mol})\end{array}$ \\
\hline PEF & $2.57 \%$ & $15.3 \mathrm{k}$ & 44.1 & $14.4 \mathrm{k}$ & $50.1 \mathrm{k}$ \\
\hline ECP5 & $5.52 \%$ & $15.7 \mathrm{k}$ & $29.8 k$ & $6.1 \mathrm{k}$ & $27.6 \mathrm{k}$ \\
\hline ECP9 & $11.94 \%$ & $7.3 \mathrm{k}$ & $12.0 \mathrm{k}$ & $5.9 \mathrm{k}$ & $23.8 \mathrm{k}$ \\
\hline$E C P 24$ & $21.01 \%$ & $11.0 \mathrm{k}$ & $18.9 \mathrm{k}$ & $0.8 \mathrm{k}$ & $23 \mathrm{k}$ \\
\hline ECP43 & $37.17 \%$ & $8.6 \mathrm{k}$ & $14.8 \mathrm{k}$ & $0.4 \mathrm{k}$ & $17.8 \mathrm{k}$ \\
\hline ECP82 & $76.26 \%$ & $3.1 \mathrm{k}$ & $6.3 \mathrm{k}$ & - & - \\
\hline
\end{tabular}


Gas barrier properties

Polymer films with a diameter of $\sim 3 \mathrm{~cm}$ were prepared from the pure PEF sample and from copolymers ECP5, and ECP24 by vacuum-melt pressing and subsequent quenching in between kapton sheets (SI). The prepared films of copolymers were brittle and were difficult to handle, due to which a consistent gas barrier properties measurement on these films proved challenging. Most likely the molecular weight of the prepared polymers was too low to have sufficient melt strength to facilitate good film preparation. Furthermore, large scale polymerization of these samples was restricted due to the limited availability of 1,3-CPdiol as monomer, and thereby limiting the size (diameter) of the polymer films. To this end, rather than using sheets having $9.5 \mathrm{~cm}$ diameter, samples having a diameter of $2.5 \mathrm{~cm}$ were prepared to measure the gas barrier properties (SI). Unfortunately, the decrease in sample area inherently leads in a lower total gas transport (in moles) through the film, and for the films of PEF and ECP5 the total gas transport was lower than the lower detection limit of the apparatus used at the small sample area. Despite the abovementioned limitations in the preparation of these polymer films, preliminary Oxygen Transmission Rate (OTR) values were obtained at $23{ }^{\circ} \mathrm{C}$ on PEF, ECP5, and ECP24 (average test area of $3.66 \mathrm{~cm}^{2}$ ) and a Relative Humidity $(\mathrm{RH})$ of 50\% (Table 3-5).

Table 3-5. Obtained oxygen transmission rates for (co)polymer films of PEF, ECP5, and ECP24.

\begin{tabular}{c|c|c|} 
Sample & OTR $\left(\mathrm{cm}^{3} / \mathrm{m}^{2} / 24 \mathrm{~h} / \mathrm{atm}\right)$ & \# samples \\
\hline PEF & $<0.07$ & 1 \\
\cline { 2 - 3 } ECP5 & $<0.07$ & 2 \\
\cline { 2 - 3 } ECP24 & 0.34 & 1 \\
\cline { 2 - 3 } & &
\end{tabular}

The films of PEF and ECP5 had an OTR value of $<0.07 \mathrm{~cm}^{3} / \mathrm{m}^{2} / 24$ hours, which is lower than the detection limit of the apparatus used, yet the inclusion of $5 \%$ CPdiol does not seem to affect the good barrier properties of PEF significantly. More importantly, ECP24 exhibited a strongly increased OTR value of $0.34 \mathrm{~cm}^{3} / \mathrm{m}^{2} / 24$ hours/atm, which is at least five-fold increase in oxygen transport through the polymer film as compared to EPF and ECP5. From these preliminary studies it is clear that copolymerization of CPdiol in PEF increases the diffusion rate of oxygen through the polymer significantly. Therefore, the high BIF values PEF has as compared to PET will be reduced, much similar to PEF copolymers containing other cyclic diols, as summarized in Table 3-1. 


\section{Conclusions}

Copolymerization of PEF with potentially renewable cycloaliphatic 1,3-cyclopentanediol via standard polycondensation methods is described for the first time. In total five copolymers are synthesized with $5,9,24,43$, and $82 \%$ of $1,3-C P$ diol respectively. A general trend could be observed that with the higher content of CPdiol, the lower the $T_{\mathrm{g}}$, which is in contrast to most commercial and industrial relevant cyclic diols for which copolymerization often leads to an increase in $T_{\mathrm{g}}$. Furthermore, inclusion of CPdiol hampered crystallization of PEF as seen by the lowering in melting temperature, indicating CPdiol cannot co-crystallize with PEF, which is normal for the copolymerization of cyclic diol. Exception is the copolymer having $82 \%$ of $\mathrm{CPdiol}$, which though crystalline is thermally unstable in the melt above $255^{\circ} \mathrm{C}$, which is caused by the inherent instability of CPdiol above $240{ }^{\circ} \mathrm{C}$. Further TGA studies of the obtained copolymers when left in the melt at $240{ }^{\circ} \mathrm{C}$ for an hour showed that with increasing CPdiol content, the thermal stability decreases, leading to completely cross-linked insoluble black particles in the copolyesters having more than $24 \%$ of CPdiol. Preliminary gas barrier properties on amorphous films of PEF and copolymers with $5 \%$ and $25 \%$ CPdiol were measured on a film having a diameter of $2.5 \mathrm{~cm}$. Though the prepared polymer films were not ideal, preliminary values indicate that inclusion of CPdiol leads to an increase in OTR, similar to previously reported copolymers of PEF with cyclic diols. When comparing the copolymers of PEF with CPdiol to commercially relevant PEF copolymers with cyclic diols, CPdiol behaves similarly in terms of suppressing crystallinity and an increase in OTR. However, CPdiol decreases the $T_{\mathrm{g}}$ of PEF, which is likely caused by the less stable 5-membered cyclopentane ring, as compared to the highly stable 6-membered cyclohexane rings. CPdiol does not facilitate the usual trade-off between increase in $T_{\mathrm{g}}$ for barrier properties, but hampers both. Together with the inherent instability at elevated temperature, CPdiol does not seem viable as a renewable alternative for cyclic diol comonomers for the preparation of products especially where high impact strength is desired e.g. bottle-grade PEF.

\section{Conflicts of interest}

There are no conflicts to declare.

\section{Acknowledgements}

This work was performed under the framework of Chemelot InSciTe and is supported by contributions from the European Regional Development Fund (ERDF) within the framework of OP-Zuid and with contributions from the province of Brabant and Limburg and the Dutch Ministry of Economy. 


\section{References}

1. de Jong, E., Dam, M. a., Sipos, L. \& Gruter, G.-J. M. Furandicarboxylic Acid (FDCA), A Versatile Building Block for a Very Interesting Class of Polyesters. in ACS Symposium Series 1105, 1-13 (2012).

2. Burgess, S. K. et al. Chain Mobility, Thermal, and Mechanical Properties of Poly(ethylene furanoate) Compared to Poly(ethylene terephthalate). Macromolecules 47, 1383-1391 (2014).

3. Burgess, S. K., Karvan, O., Johnson, J. R., Kriegel, R. M. \& Koros, W. J. Oxygen sorption and transport in amorphous poly(ethylene furanoate). Polymer (Guildf). 55, 4748-4756 (2014).

4. Burgess, S. K. et al. Water sorption in poly(ethylene furanoate) compared to poly(ethylene terephthalate). Part 1: Equilibrium sorption. Polymer (Guildf). 55, 6861-6869 (2014).

5. Burgess, S. K. et al. Water sorption in poly(ethylene furanoate) compared to poly(ethylene terephthalate). Part 2: Kinetic sorption. Polymer (Guildf). 55, 6870-6882 (2014).

6. Burgess, S. K., Kriegel, R. M. \& Koros, W. J. Carbon Dioxide Sorption and Transport in Amorphous Poly(ethylene furanoate). Macromolecules 48, 2184-2193 (2015).

7. Burgess, S. K., Wenz, G. B., Kriegel, R. M. \& Koros, W. J. Penetrant transport in semicrystalline poly(ethylene furanoate). Polymer (Guildf). 98, 305-310 (2016).

8. Sun, L. et al. New insight into the mechanism for the excellent gas properties of poly(ethylene 2,5-furandicarboxylate) (PEF): Role of furan ring's polarity. Eur. Polym. J. 118, 642-650 (2019).

9. Ahn, J. S. et al. Effect of alicyclic monomers on thermal properties of transparent biodegradable polyesters. Macromol. Res. 24, 609-616 (2016).

10. Wang, J., Liu, X., Zhang, Y., Liu, F. \& Zhu, J. Modification of poly(ethylene 2,5-furandicarboxylate) with 1,4cyclohexanedimethylene: Influence of composition on mechanical and barrier properties. Polymer (Guildf). 103, 1-8 (2016).

11. Wang, J. et al. Synthesis of bio-based poly(ethylene 2,5-furandicarboxylate) copolyesters: Higher glass transition temperature, better transparency, and good barrier properties. J. Polym. Sci. Part A Polym. Chem. 55, 3298-3307 (2017).

12. Wang, J. et al. Biobased Amorphous Polyesters with High T g: Trade-Off between Rigid and Flexible Cyclic Diols. ACS Sustain. Chem. Eng. 7, 6401-6411 (2019).

13. Chen, T., Jiang, G., Li, G., Wu, Z. \& Zhang, J. Poly(ethylene glycol-co-1,4-cyclohexanedimethanol terephthalate) random copolymers: effect of copolymer composition and microstructure on the thermal properties and crystallization behavior. RSC Adv. 5, 60570-60580 (2015).

14. Quintana, R., de llarduya, A. martínez, Alla, A. \& Muñoz-Guerra, S. Polyterephthalates made from Ethylene glycol, 1,4cyclohexanedimethanol, and isosorbide. J. Polym. Sci. Part A Polym. Chem. 49, 2252-2260 (2011).

15. Yoon, W. J. et al. Synthesis and Characteristics of a Biobased High- T g Terpolyester of Isosorbide, Ethylene Glycol, and 1,4-Cyclohexane Dimethanol: Effect of Ethylene Glycol as a Chain Linker on Polymerization. Macromolecules 46, 7219-7231 (2013).

16. Kelsey, D. R., Scardino, B. M., Grebowicz, J. S. \& Chuah, H. H. High impact, amorphous terephthalate copolyesters of rigid 2,2,4,4-tetramethyl-1,3-cyclobutanediol with flexible diols. Macromolecules 33, 5810-5818 (2000).

17. Stanley, N. et al. Bimetallic Catalytic Systems Based on Sb, Ge and Ti for the Synthesis of Poly(ethylene terephthalateco-isosorbide terephthalate). Polymers (Basel). 9, 590 (2017).

18. Muñoz-Guerra, S., Lavilla, C., Japu, C. \& Martínez de llarduya, A. Renewable terephthalate polyesters from carbohydrate-based bicyclic monomers. Green Chem. 16, 1716-1739 (2014).

19. Lavilla, C., Martínez de llarduya, A., Alla, A. \& Muñoz-Guerra, S. PET copolyesters made from a d-mannitol-derived bicyclic diol. Polym. Chem. 4, 282-289 (2013).

20. Japu, C., Martínez de Ilarduya, A., Alla, A. \& Muñoz-Guerra, S. Bio-based poly(ethylene terephthalate) copolyesters made from cyclic monomers derived from tartaric acid. Polymer (Guildf). 55, 2294-2304 (2014).

21. Gomes, M., Gandini, A., Silvestre, A. J. D. \& Reis, B. Synthesis and characterization of poly(2,5-furan dicarboxylate)s based on a variety of diols. J. Polym. Sci. Part A Polym. Chem. 49, 3759-3768 (2011).

22. Papageorgiou, G. Z., Papageorgiou, D. G., Terzopoulou, Z. \& Bikiaris, D. N. Production of bio-based 2,5-furan dicarboxylate polyesters: Recent progress and critical aspects in their synthesis and thermal properties. Eur. Polym. J. 83, 202-229 (2016).

23. Wang, J., Liu, X., Zhu, J. \& Jiang, Y. Copolyesters Based on 2,5-Furandicarboxylic Acid (FDCA): Effect of 2,2,4,4Tetramethyl-1,3-Cyclobutanediol Units on Their Properties. Polymers (Basel). 9, 305 (2017).

24. Hong, S., Min, K.-D., Nam, B.-U. \& Park, O. O. High molecular weight bio furan-based co-polyesters for food packaging applications: synthesis, characterization and solid-state polymerization. Green Chem. 18, 5142-5150 (2016).

25. Wang, J. et al. Modification of poly(ethylene 2,5-furandicarboxylate) (PEF) with 1, 4-cyclohexanedimethanol: Influence of stereochemistry of 1,4-cyclohexylene units. Polymer (Guildf). 137, 173-185 (2018).

26. Wang, J., Liu, X., Jia, Z., Sun, L. \& Zhu, J. Highly crystalline polyesters synthesized from furandicarboxylic acid (FDCA): Potential bio-based engineering plastic. Eur. Polym. J. 109, 379-390 (2018).

27. Kim, T. et al. Sustainable terpolyester of high T g based on bio heterocyclic monomer of dimethyl furan-2,5-dicarboxylate and isosorbide. Polymer (Guildf). 132, 122-132 (2017).

28. Noordzij, G. J., Dietz, C. H. J. T., Leoné, N., Wilsens, C. H. R. M. \& Rastogi, S. Small-scale screening of novel biobased monomers: the curious case of 1,3-cyclopentanediol. RSC Adv. 8, 39818-39828 (2018).

29. Li, G. et al. Industrially scalable and cost-effective synthesis of 1,3-cyclopentanediol with furfuryl alcohol from lignocellulose. Green Chem. 18, 3607-3613 (2016).

30. Matos, M., Sousa, A. F. \& Silvestre, A. J. D. Improving the Thermal Properties of Poly(2,5-furandicarboxylate)s Using Cyclohexylene Moieties: A Comparative Study. Macromol. Chem. Phys. 218, 1600492 (2017). 


\section{Supplementary information for chapter 3}

\section{NMR-analysis}

The successful copolymerization between 2,5-FDCAdm, EG, and CPdiol is shown via NMR analysis. 2D-analysis of ECP43 is shown in figures 1-3 to assign the right peaks. Table 1 shows the calculated ratio between EG (signal 2, $4.7 \mathrm{ppm}$ ) and CPdiol (signal 5, $5.5 \mathrm{ppm}$ ), which after normalization to total $\mathrm{H}$ of the signal (signal $\mathbf{2}=4 \mathrm{H}$, signal $5=2 \mathrm{H}$ ), give the ratio. Polymer samples are named after the calculated ratio of CP, e.g. ECP43 has $43 \mathrm{~mol} \% \mathrm{CP}$ and $57 \mathrm{~mol} \% \mathrm{EG}$. The copolyesters contain an unidentified peak at ${ }^{1} \mathrm{H} 1.43 \mathrm{ppm},{ }^{13} \mathrm{C} 30.1 \mathrm{ppm}$, however in COSYNMR it shows no neighboring signals, so it seems unattached the polymer and might be an impurity in the NMR solvent.

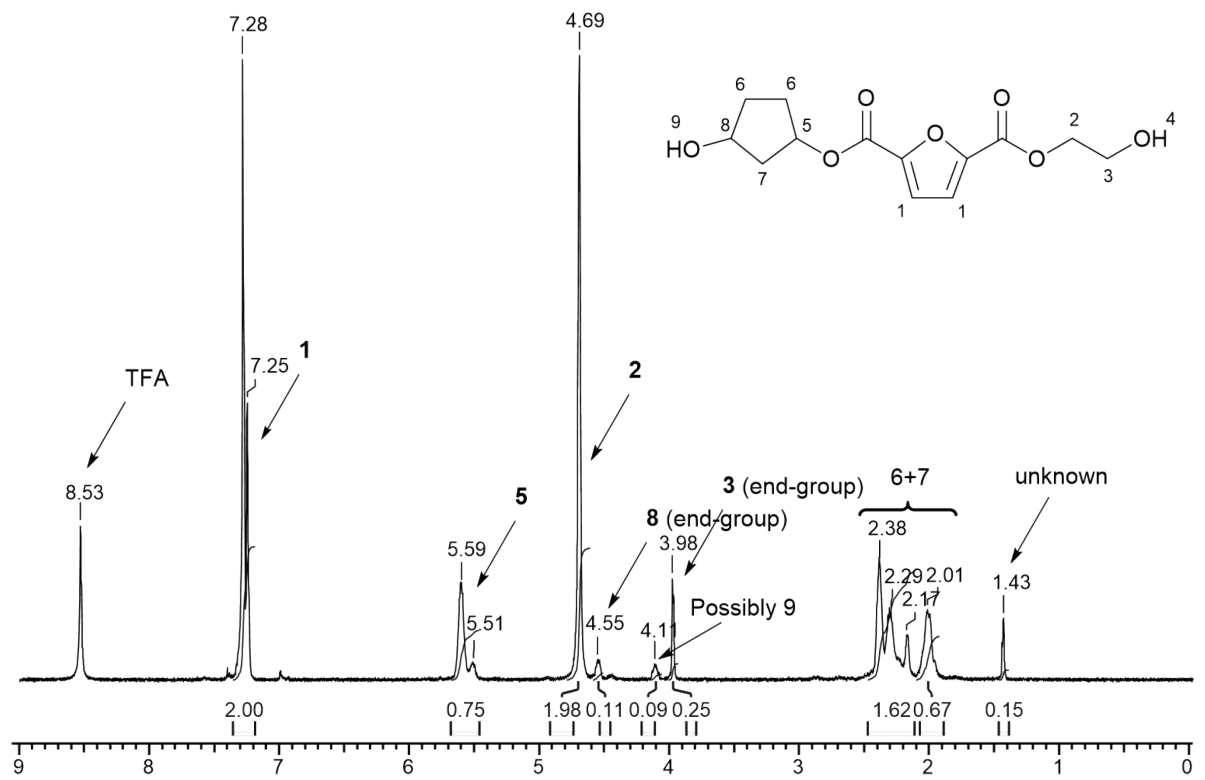

Figure S3 - 1. ${ }^{1} \mathrm{H}-\mathrm{NMR}$ analysis of copolyester ECP43\%. 


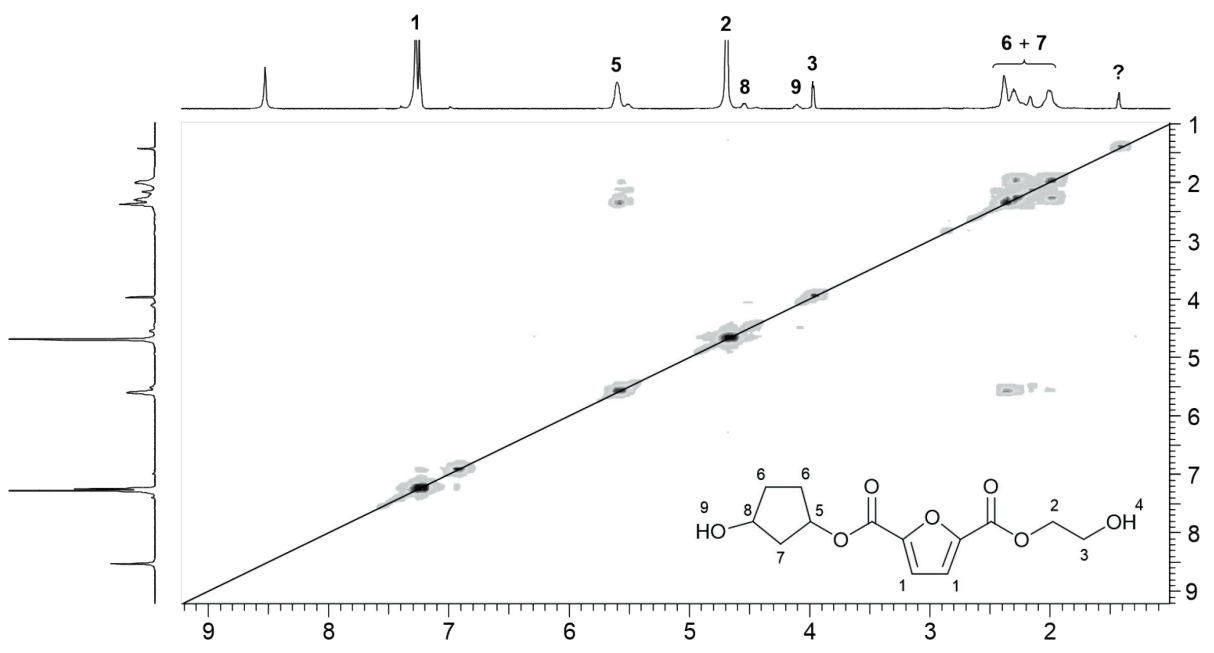

Figure $\mathrm{S} 3$ - 2. COSY $\left({ }^{1} \mathrm{H}-{ }^{1} \mathrm{H}\right)$ NMR analysis of copolyester ECP43\%.

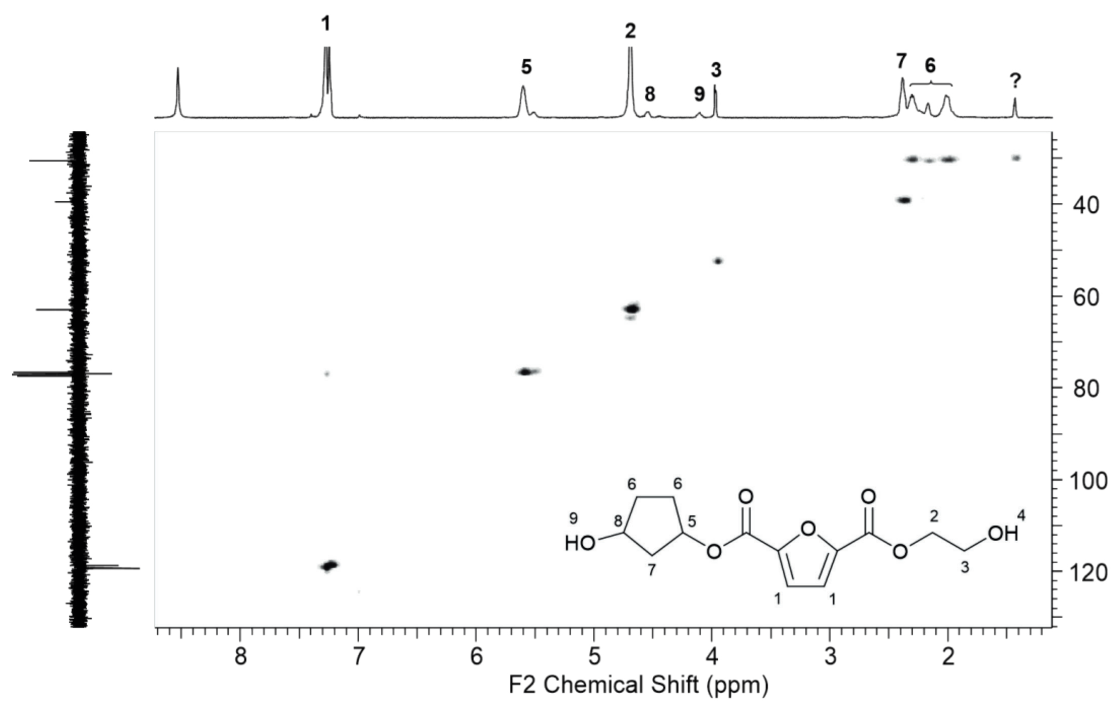

Figure S3 - 3. HSQC $\left({ }^{1} \mathrm{H}-{ }^{13} \mathrm{C}-\mathrm{APT}\right.$ analysis of copolyester ECP $43 \%$. 
Table S3 - 1. Calculated ratios of actual CP-content in copolyesters. The ratio of polymeric (non end-group) EG (signal 2, $4.7 \mathrm{ppm}$ ) vs. polymeric CP (signal $5,5.5 \mathrm{ppm}$ ).

\begin{tabular}{c|c|c|c|c|c|} 
Sample & $\begin{array}{c}\text { Signal EG } \\
(\mathbf{4 . 7} \mathbf{~ p p m})\end{array}$ & $\begin{array}{c}\text { Signal CP } \\
\mathbf{( 5 . 5} \mathbf{~ p p m})\end{array}$ & \multicolumn{2}{c}{ Per H atom E } & \multicolumn{2}{c}{ Per H atom CP } & $\begin{array}{c}\text { \% CP via } \\
\text { CP/(EF+CP) }\end{array}$ \\
\hline PEF & 3.71 & 0 & 0.923 & 0 & $0 \%$ \\
\cline { 2 - 6 } ECP5 & 3.53 & 0.09 & 0.883 & 0.045 & $5 \%$ \\
\cline { 2 - 6 } ECP9 & 3.05 & 0.15 & 0.763 & 0.075 & $9 \%$ \\
\cline { 2 - 6 } ECP24 & 2.08 & 0.33 & 0.52 & 0.165 & $24 \%$ \\
\cline { 2 - 6 } ECP43 & 1.98 & 0.75 & 0.495 & 0.375 & $43 \%$ \\
\cline { 2 - 6 } ECP82 & 0.57 & 1.28 & 0.143 & 0.64 & $82 \%$ \\
\hline
\end{tabular}

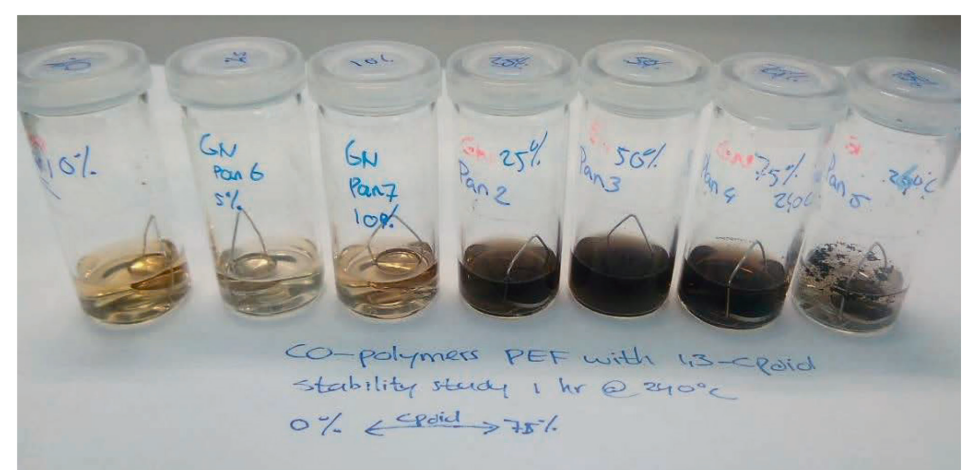

Figure S3 - 4. Dissolution of the copolymers in HFIP after an hour isothermal step at $240{ }^{\circ} \mathrm{C}$ in the TGA. From left to right: PEF, ECP5, ECP9, ECP24, ECP43, ECP82. Far right: ECP82 after an hour isothermal step at $260{ }^{\circ} \mathrm{C}$ in the TGA. The soluble fractions were taken to measure the molecular weight in HFIP-GPC.
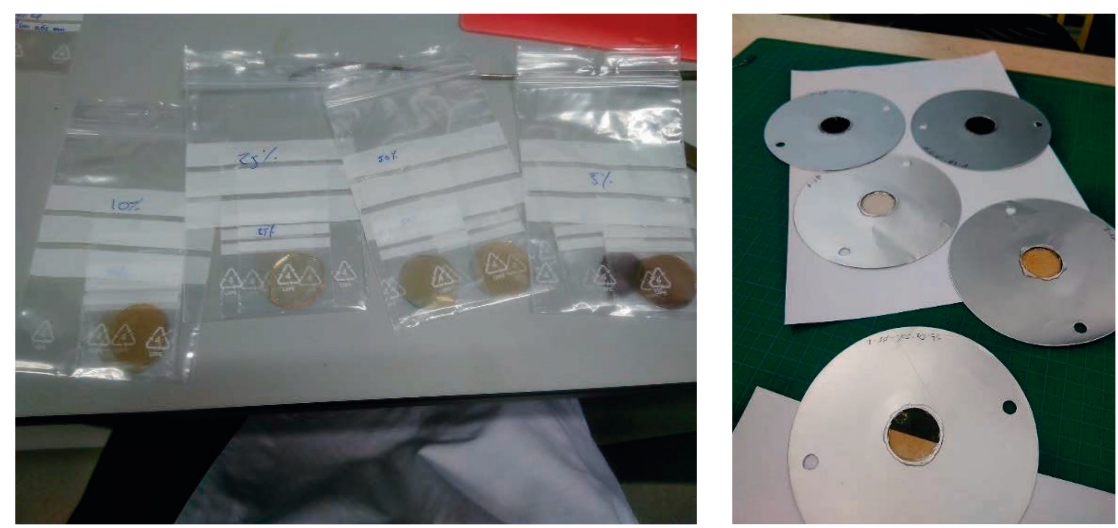

Figure S3 - 5. Left: Vacuum-pressed amorphous copolyester films of $\sim$ ø $\mathrm{cm}$. ECP5 = 5\%, ECP9 = $10 \%, \mathrm{ECP} 24=25 \%, \mathrm{ECP} 43=50 \%$. ECP82 could not be made due to instable melt. Right: Polyester films in between two sheet holders with a $\varnothing$ of $2.5 \mathrm{~cm}$, the edges on the outside are glued with twocomponent glue to avoid leakage between the sheets and polymer films. 


\section{4}

\section{Chapter 4 Cascade aza-Michael addition- cyclizations}

This chapter is published as:

Cascade aza-Michael addition-cyclizations: Towards renewable and multifunctional carboxylic acids for melt-polycondensation

G.J. Noordzij, ${ }^{a, b}$ C.H.R.M. Wilsens, ${ }^{b}$

Frontiers in Chemistry, 2019. Part of research topic: Sustainable Polymers: Where Are We? Where Do We Go?

DOI: 10.3389/fchem.2019.00729

${ }^{a}$ Chemelot InSciTe, Urmonderbaan 20F, NL-6167 RD Geleen, The Netherlands.

${ }^{b}$ Aachen-Maastricht Institute of Biobased Materials (AMIBM), Faculty of Science and Engineering, Maastricht University, Brightlands Chemelot Campus, 6167 RD Geleen, The Netherlands.

This chapter includes part of the bachelor thesis research of Catherine Gilbert.

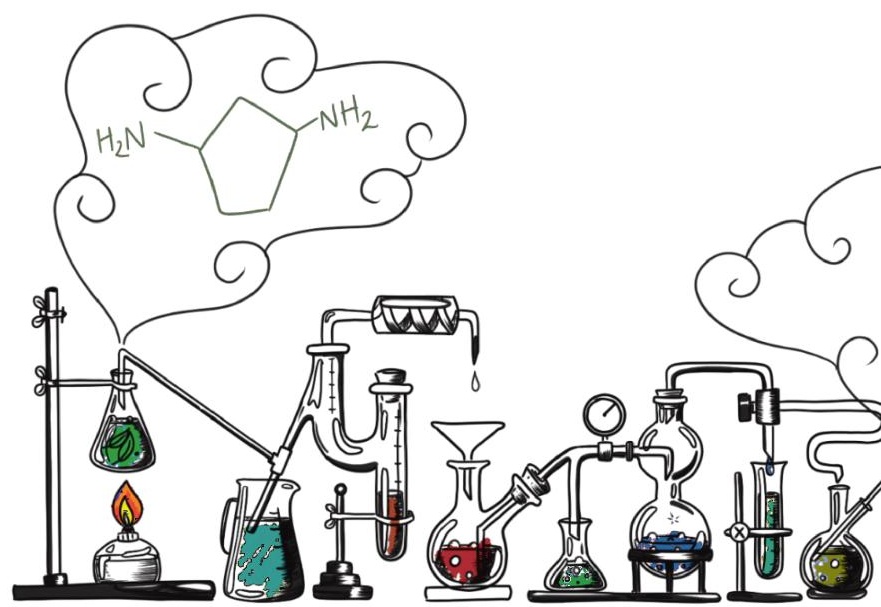




\section{Introduction}

Recently, polymeric materials are increasingly (re-)evaluated in academia, industry, and society as they are reportedly causing pollution and negatively impact the environment. ${ }^{1}$ One route to improve the sustainability and environmental impact is to consider the principles of green chemistry, both for the development of monomers and for the development of polymer materials. Example of components that need to be considered are the atom efficiency, use of renewable feedstock, design to recycle, use of lesshazardous chemical synthesis, solvents, and catalysts. ${ }^{2}$ Particularly interesting transformations that can be considered green reactions are click-reactions, as is coined by Sharpless and coworkers in $2001 .^{3}$ One of such click-reactions in organic chemistry is the well-known aza-Michael reaction (Scheme 4-1), which is an addition reaction involving a nucleophilic amine (Michael donor), and an electron deficient alkene (Michael acceptor). This reaction is widely employed in the synthesis of biologically active compounds and, when using the right reactants, readily proceeds at room temperature. ${ }^{4}$

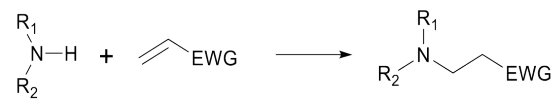

Scheme 4-1. Generalized reaction scheme of the aza-Michael addition reaction between a primary $\left(R_{1}=H, R_{2} \neq H\right)$, or a secondary amine $\left(R_{1}, R_{2} \neq H\right)$, and an electron deficient amine with an electron withdrawing group (EWG).

Not surprisingly, the aza-Michael addition has been used in both monomer as polymer synthesis, and has been reviewed in three recent publications regarding the use of the aza-Michael reaction applied to the general polymer domain $(2006)^{5}$, to the general chemistry domain $(2011)^{4}$, and to silicon-based polymers (2017). ${ }^{6}$ The aza-Michael addition reaction is particularly interesting for modification of renewable monomers, as numerous renewable molecules contain primary or secondary amines and/or unsaturated groups. Furthermore, the selectivity of the aza-Michael addition reaction can be used to convert multi-functional molecules into new biobased monomers for polymerization reactions. One example of such monomer is itaconic acid or its methyl ester, dimethyl itaconate, which can function as a Michael acceptor. This molecule is prone to participate in the aza-Michael addition reaction, and subsequently undergoes a cascade cyclization reaction to form a highly stable $\mathrm{N}$-alkyl-pyrrolidone carboxylic acid. ${ }^{7}$ In other words, this reaction allows for the transformation of an amine group into a rigid and thermally stable carboxylic acid functionalized monomer, which can be used for further polymerization reactions.

The previously described cascade cyclization reaction to form new stable monomeric intermediates via the aza-Michael reaction has not been described in previous reviews. This is unexpected, as, besides itaconic acid, several other monomers such as fumaric acid, maleic acid, trans- $\beta$-hydromuconic, and aconitic acid, prove susceptible to participate in the aza-Michael (cascade) reaction to yield highly promising monomers for further polymerization. Therefore, in this work we provide an overview of the current research activities on renewable unsaturated esters for monomer synthesis via both the 
aza-Michael addition and the cascade cyclization reaction, and elaborate on their use in melt-polycondensation reactions. Furthermore, we extend the state-of-the-art with new findings, highlighting the potential of the aza-Michael cascade reaction for the monomer aconitic acid and demonstrate its potential in polymerization reactions.

\section{Essentials for aza-Michael cascade reaction.}

\section{Michael donors}

The reactivity of different amines as Michael donor has been studied for several Michael acceptors, such as acrylates ${ }^{8}$, methyl acrylates ${ }^{9}$, and vinyl phosphonates ${ }^{10}$. Several factors influence the reactivity of the amine as Michael donor, such as nucleophilicity, steric hindrance, and aromaticity. In general, cyclic secondary amines such as piperidine are stronger nucleophiles than primary amines due to the inductive effect of the $N$ substituted alkyl groups, and can therefore react faster as Michael donor. However, bulky secondary amines such as di-butyl-amine are slower to react than primary amines due to steric hindrance. In turn aromatic amines such as aniline showed no reactivity as Michael donor at room temperature without catalyst, and therefore would require harsher conditions to react. ${ }^{11} \mathrm{~A}$ comprehensive overview of the different reactivity of amines as Michael donor has been extensively discussed in an excellent review paper by Ganachaud and coworkers and we refer to this work for more information on this topic. ${ }^{6}$

\section{Michael acceptors}

The capability of unsaturated monomers to act as Michael acceptor depends on the nature of this unsaturated bond. Only electron deficient unsaturated bonds are capable of accepting an electron from the nucleophilic Michael donor, and the degree of electron deficiency is largely determined by electron withdrawing groups (EWG) on the $\beta$-position relative to the unsaturation. It was shown that nitrile groups and methyl esters have the highest electron withdrawing effect (Scheme 4-2) ${ }^{12}$, and therefore unsaturated monomers with these EWGs in the $\beta$-position are highly susceptible towards to azaMichael addition reaction. This can be seen by the high amount of publications of the aza-Michael addition on unsaturated monomers such as acrylates and acrylonitriles. ${ }^{6}$

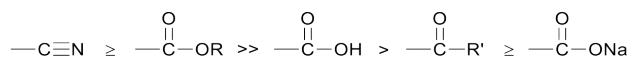

Scheme 4-2. From left to right: the strongest electron withdrawing group to less strong electron withdrawing group. The stronger the electron withdrawing group on the $\beta$-position of the unsaturated bond, the higher the reactivity towards the aza-Michael addition reaction.

The electron deficiency of the unsaturation is also dependent of the degree of alkylsubstitution on the monomer. Alkyl substitutions, which have an electron donating effect, on both the $\alpha-$, as $\beta$-position can slow down the aza-Michael addition reaction (Scheme 4-3). ${ }^{6}$ Generally it is reported that with a higher degree of alkyl-substitution, an increase in reaction temperature was required for the aza-Michael addition to occur. ${ }^{12}$ 


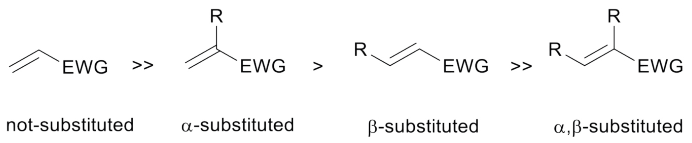

Scheme 4-3. The reactivity of the Michael acceptor is dependent on the degree of $\alpha, \beta$-substitution.

\section{Cascade cyclization reaction}

Apart from the aza-Michael addition reaction, monomers with an ester group on the $\gamma^{-}$ position relative to the unsaturated bond can undergo a cascade reaction when ammonia or a primary amine is used in the aza-Michael addition reaction. The formed secondary amine in the aza-Michael adduct (Scheme 4-4, middle) can act as a nucleophile, and undergoes an autocatalyzed intramolecular amidation-cyclization reaction with an ester spaced four carbons away ( $\mathrm{Y}$-position) to form a highly stable 5-membered $\mathrm{N}$-substituted pyrrolidone ring (Scheme 4-4, right). This cascade reaction is well known to occur with the biobased monomer dimethyl itaconate (Scheme 4-4, EWG = methyl-ester, $R_{1}=H$ ), and readily reaches full conversion when conducted at elevated temperatures, as will be explained in more detail later in this work. The cyclization reaction is irreversible under normal synthesis conditions, and thereby avoids any potential retro aza-Michael reactions which have been observed for some systems. ${ }^{13}$ Although the cyclization step does include the loss of a condensation molecule, the reaction can proceed fast, highly selective and with high yields, both in bulk or in green solvents such as methanol or water, hence following the principles of green chemistry.

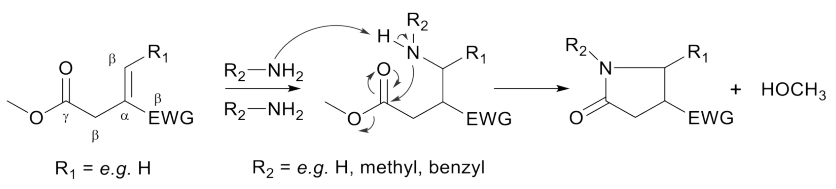

Scheme 4-4. Schematic overview of the cascade reaction of the aza-Michael addition of a primary amine to an unsaturated monomer with an ester in the y-position (left). The aza-Michael addition reaction is followed by an autocatalyzed amidation-cyclization step (middle, reaction arrows) which, with the loss of a condensate molecule, forms a 5 -membered $N$-substituted pyrrolidone ring (right). 


\section{Renewable monomer selection}

Renewable monomers with an unsaturation are widely reported and examples include biobased acrylates, terpenes, furfural/furfuryl alcohol based monomers, glycerides and related unsaturated fatty acids, and unsaturated esters. As described in the previous section, the electron deficiency of the double bond is crucial in order for these molecules to be a candidate for the aza-Michael addition reaction. Monomers without EWG in close vicinity to the unsaturation are typically unreactive towards the aza-Michael addition, as is the case for unsaturated fatty acids. Some lignin-derived and terpene-derived unsaturated monomers do have vicinal EWGs but usually lack additional functional groups apart from an unsaturated bonds, thereby often limiting their use to radical polymerizations. ${ }^{14,15}$ Conjugated or aromatic unsaturated double bonds such as in muconic acid, 2,5-furandicarboxylic acid, 2,5-hydroxylmethylfurfural, furfuryl, and furfurylalcohol are greatly stabilized by conjugation, and thereby have no reported reactivity towards the aza-Michael addition reaction. Furthermore, 2,5-hydroxymethylfurufral, furfurylalcohol, and some of their derivatives have functional groups (e.g. aldehydes) which reacts faster with amines, thereby competing with, or even overruling the aza-Michael addition reaction.

One class of biobased unsaturated monomers which are (highly) susceptible towards aza-Michael addition reactions are unsaturated (di-)esters. Importantly, unsaturated (di)esters can retain multi-functionality after the aza-Michael addition with mono- or diamines, and can therefore subsequently be used in the preparation of linear, branched, and cross-linked polymers. Since to date this part has not received much attention in academic literature, in the next section we provide an overview on the aza-Michael addition reaction involving unsaturated (di-)esters from biomass, with a special attention for the potential use in the synthesis of (biobased) polymers. Note, several relevant unsaturated esters from biomass have been excluded from this overview, the reason being that (potentially) biobased acrylates, obtainable via glycerol ${ }^{16}$, have already been included in a review regarding the aza-Michael additions on acrylate monomers. ${ }^{6}$

The aforementioned selection criteria on reactivity leaves several candidates which are proven to be susceptible, or expected to be susceptible, towards the aza-Michael addition reaction under mild reaction conditions (Scheme 4-5). Firstly, aconitic acid (cis and trans) is a tri-functional carboxylic acid with an unsaturation in the backbone, and can be obtained as a by-product form the sugar-cane industry ${ }^{17}$, or after dehydration of biobased citric acid. Further dehydration of aconitic acid leads to the corresponding cis and trans aconitic anhydrides, with the unsaturation retained within the anhydride ring, or in the side chain. Thermal treatment of these anhydrides can lead to decarboxylation to form citraconic anhydride from cis-aconitic anhydride, or itaconic anhydride from trans-aconitic anhydride. 


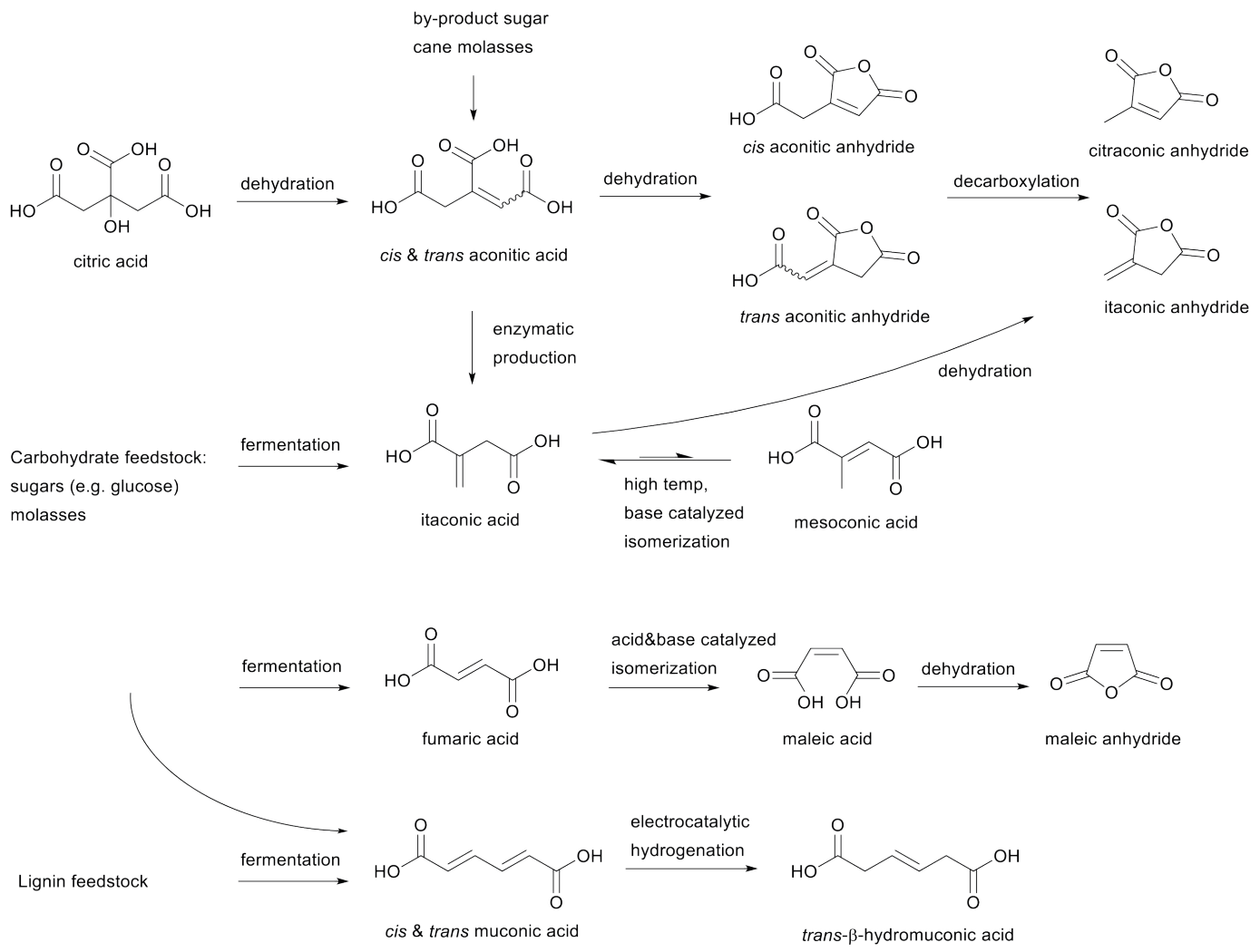

Scheme 4-5. Overview scope of literature research of unsaturated esters from biomass for the aza-Michael addition reaction.

Itaconic acid, an unsaturated ester with the unsaturation exo on the backbone can readily be obtained from fermentation of several carbohydrate feedstocks such as glucose, but also from aconitic acid. Itaconic acid is produced on an industrial scale, and is for example used in superabsorbent polymers by Itaconix ${ }^{\circledR}$. Itaconic acid can be base catalyzed at elevated temperatures to isomerize towards mesaconic acid, and dehydration of itaconic acid can lead to itaconic anhydride as shown before in the aconitic acid cycle.

Other relevant biobased unsaturated esters are fumaric and maleic acid, which can be obtained by fermentation of carbohydrate biomass feedstocks. ${ }^{18}$ Maleic acid is the structural isomer of fumaric acid and can be obtained via both acid as base catalyzed isomerization. Furthermore, maleic acid can be dehydrated to form maleic anhydride.

Lastly, muconic acid, a C6 acid with a double unsaturation in the main chain, can be obtained from fermentation of both carbohydrate feedstocks as from lignin feedstocks. ${ }^{19}$ Electrochemical hydrogenation of muconic acid leads to trans- $\beta$-hydromuconic acid, with one unsaturation left in the main chain. Though currently commercially not yet relevant, in recent years it has gained scientific interest as muconic acid provides a potential route 
for the production of biobased terephthalic acid ${ }^{20}$, and trans- $\beta$-hydromuconic acid for adipic acid. ${ }^{21,22}$

\section{State of the Art}

\section{Aza-Michael addition on itaconic acid}

Itaconic acid and dimethyl itaconate are highly susceptible towards the aza-Michael addition reaction, and, when reacted with secondary amines, form a good platform to modify the backbone whilst retaining the dimethylcarboxylate functionality. For example, the addition of diethylamine to itaconate is highly selective towards the $\beta$-position, and therefore adds only to the exo-position. ${ }^{23}$ This modification can be performed both prior to polymerization of the itaconates ${ }^{24}$, or after polymerization (Scheme 4-6, top). ${ }^{25,26}$ Furthermore, the aza-Michael addition reaction is reported to be irreversible at the employed reaction conditions (room temperature), and only showed minor reversibility at elevated temperatures. ${ }^{24}$ The potential aza-Michael addition reaction with mesaconate does not occur at all, which shows that, despite the isomerization of itaconate to mesaconate, the aza-Michael reaction is specific to the $\beta$-position on itaconates. Nevertheless, Clark and coworkers reported that eventually all mesaconate isomerizes back to itaconate when the reaction was left stirring long enough, which then formed the aza-Michael adduct with almost full conversion. ${ }^{23}$

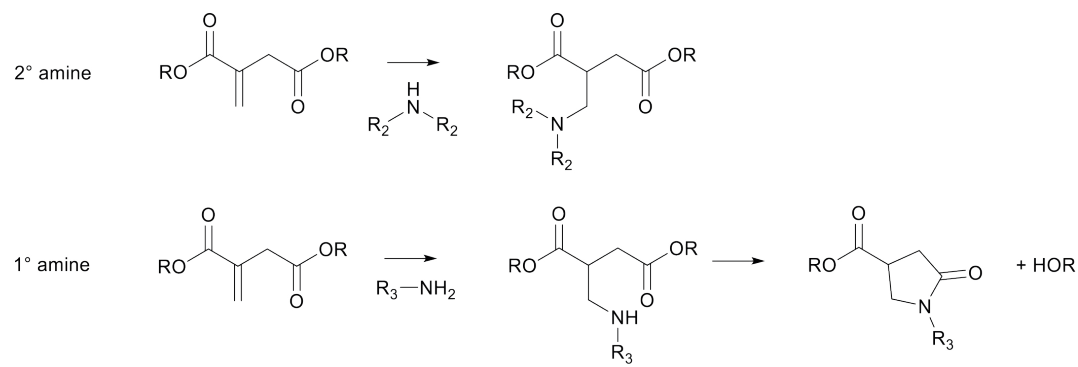

Scheme 4-6. Schematic overview of possible aza-Michael addition reactions on itaconates. Top: a secondary amine (e.g. diethylamine, piperidine) can add to itaconates to form the aza-Michael adduct. Middle: primary amines (e.g. ethylamine, ethanolamine) can add to itaconates to form the aza-Michael adduct which subsequently ring-closes to form $\mathrm{N}$-functionalized mono-pyrrolidone.

The aza-Michael addition reaction between itaconic acid and aliphatic primary amines is well-known to subsequently undergo a cascade intramolecular cyclization step to form a $N$-substituted pyrrolidone ring (Scheme 4-6, bottom, $\mathrm{R}_{3}=$ alkyl). This has for example been used to make $N$-methyl-pyrrolidone using methyl-amine, or to make surfactants with amines with long aliphatic chains. ${ }^{27,28}$ Keeping eye on further use of such monomers in polycondensation reactions, this cascade reaction is rather interesting because it is an irreversible reaction, and the newly obtained pyrrolidone-ring is thermally stable and resistant to both high and low $\mathrm{pH}$. Therefore, the stability of this newly formed group can be promising to protect otherwise labile amine and/or acid functionalities. For example, 
as is reported in previous work, the potentially renewable cis/trans 1,3cyclopentanediamines are prone to degrade (by oxidation and dehydration) at elevated temperatures, similar to their diol counterparts cis/trans 1,3-cyclopentanediols. ${ }^{29}$ This instability makes the use of these monomers via melt-polycondensation reactions challenging as the final products are heavily colored and either have low molecular weights or are cross-linked. ${ }^{29}$ Instead, when reacting these monomers with itaconic acid, thermally stable dicarboxylic acid monomers are obtained that can readily be used in melt-polycondensation reactions. ${ }^{30}$ In addition, the presence of the pyrrolidone ring adds to the rigidity of the newly obtained monomers, leading to a change in physicochemical properties.

In order for such $\mathrm{N}$-functionalized pyrrolidone-methylcarboxylates to be of use in further polymerization reactions, di- or multi-functional amines are required to retain the multifunctionality. The synthesis of such multifunctional monomers from itaconates and various amines, and the subsequent use in polymer synthesis has been known for some time, but has seen a rise in publications in the recent years. Scheme 4-7 summarizes the published itaconate-based monomer synthesis and subsequent polymerizations thereof. The reaction between itaconates and an amino-alcohol (a, e.g. with ethanolamine) leads to an carboxyl-alcohol functional pyrrolidone, which can be polymerized by selfpolycondensation (d) into an amorphous polyester with a glass transition temperature $\left(T_{\mathrm{g}}\right)$ of $60^{\circ} \mathrm{C} .{ }^{31}$ The reaction between itaconates and an amino-acid (b, e.g. with p-aminobenzoic acid or $L$-phenylalanine) leads to asymmetrical dicarboxylic acid functional pyrrolidone, which can be polymerized with a diol (e, e.g. with 1,3-propanediol) to yield amorphous polyesters with a $T_{\mathrm{g}}$ ranging between $96^{\circ} \mathrm{C}$ to $48{ }^{\circ} \mathrm{C}(\mathbf{b}=p$-aminobenzoic acid or L-phenylalanine, respectively). ${ }^{32}$

The reaction between itaconates and aliphatic diamines (c, e.g. with 1,2-ethylene diamine or 1,12-dodecanediamine) leads to symmetrical bis-pyrrolidonedimethylcarboxylate (BPDA) monomers. These BPDA monomers can be polymerized with various diols (f, e.g. with 1,3-propanediol or 1,12-dodecanediol) into amorphous polyesters with a $T_{\mathrm{g}}$ ranging from $62{ }^{\circ} \mathrm{C}$ to $-22{ }^{\circ} \mathrm{C} .{ }^{30,31}$ BPDA monomers obtained from rigid diamines (c, e.g. with $p$-phenylenediamine or trans-cyclohexanediamine) can be polymerized into semi-crystalline polyesters with various diols (f, e.g. with 1,8octanediol), with a $T_{\mathrm{g}}$ between $65^{\circ} \mathrm{C}$ and $33^{\circ} \mathrm{C}$, and a melting temperature $\left(T_{\mathrm{m}}\right)$ between $214{ }^{\circ} \mathrm{C}$ and $149{ }^{\circ} \mathrm{C} .{ }^{30}$ Furthermore, the reaction between itaconates and aliphatic or aromatic diamines (c, e.g. with 1,6-hexanediamine or p-phenylenediamine) followed by subsequent polymerization with various aliphatic or aromatic diamines (g, e.g. with 1,6hexanediamine or $p$-phenylenediamine), leads to the formation of high $T_{\mathrm{g}}$, amorphous polyamides. Such polyamides were either obtained via salt-formation between itaconic acid and diamines, with subsequent polycondensation to yield mono-pyrrolidone polyamides. ${ }^{33-37}$ Alternatively, the BPDA monomers were first isolated and subsequently polymerized via polycondensation with various amines into amorphous polyamides. ${ }^{30,38-}$ 40 
Lastly, BPDA monomer based on 1,10-dodecanediamine (c) was copolymerized into poly(ester amide)s with sebacic acid, 1,10-dodecanediamine, and 1,4-butanediol, where it was shown that adding BPDA monomer could suppress crystallization. ${ }^{41}$ BPDA monomers based on aliphatic diamines (c, e.g. is 1,2-ethylene diamine or 1,12dodecanediamine) can be polymerized with various bis(2-oxazoline)s (h, e.g. Y is 2,5furan or 1,3-phenylene) to yield amorphous poly(ester amide)s with a $T_{\mathrm{g}}$ ranging from 60 ${ }^{\circ} \mathrm{C}$ to $120{ }^{\circ} \mathrm{C} \cdot{ }^{42,43}$<smiles>[R]OC(=O)C(=C)CC(=O)OCC#CC(=O)O</smiles><smiles>[R]C(=O)C1CC(=O)N([X]O[AlH2])C1</smiles><smiles>[R]OC(=O)C(=C)CC(=O)O[R]C#CC(=O)O</smiles><smiles>[R]OC(=O)C1CC(=O)N([X])C1</smiles>

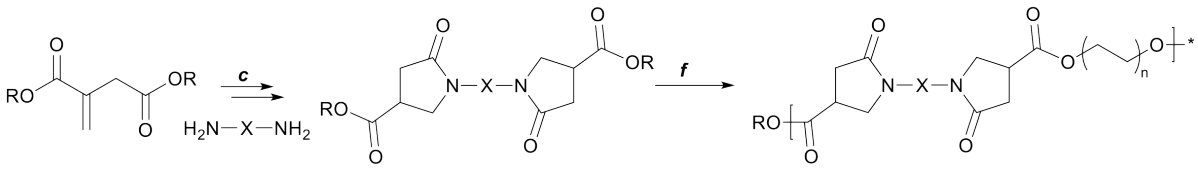

$\mathrm{R}=\mathrm{H}$ or $\mathrm{C}_{\mathrm{n}} \mathrm{H}_{2 \mathrm{n}+1}$

$\mathrm{X}=$ e.g. aliphatic or aromatic spacer

$Y=$ e.g. 2,5-furan or 1,3-phenylene
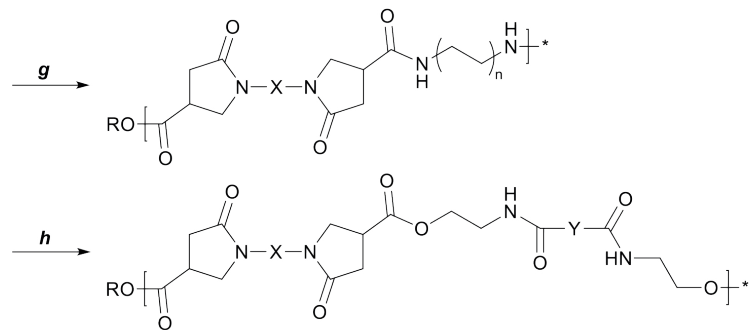

Scheme 4-7. Schematic overview of preparing polymer precursors via the aza-Michael addition reaction on itaconates. First: itaconates with amino-alcohols (a) can generate a carboxyl-alcohol functional monomers which can be used in a self-polycondensation reaction (d). Second: itaconates with amino-acids (b, e.g. p-amino-benzoic acid, phenylalanine) can generate asymmetric dicarboxylic acid functional monomers which can be polymerized with a diol (e). Third: itaconates with diamines (c, e.g. ethylene diamine, p-phenylene diamine) can generate symmetrical dimethylcarboxylate functional monomers which can be polymerized with either diols (f), diamines (g), or bisoxazolines (h) to yield polyesters, polyamides, or poly(ester amide)s, respectively.

The last member of the itaconate derivatives is itaconic anhydride, which does not show reactivity towards the aza-Michael addition reaction because anhydrides are more susceptible to undergo a reaction with amines than with the unsaturated bond. The ringopening of the anhydride results in the formation of an amide and a free carboxylic acid group (Scheme 4-8, middle left), which in turn leads to the formation of itaconimides at elevated temperatures (Scheme 4-8, middle right). Interestingly, these itaconimides retain their unsaturated bond, and several Michael additions on this double bond have been described. ${ }^{44}$ However, to date and to the best of our knowledge, no aza-Michael addition on these itaconimides has been reported in literature (Scheme 4-8, right). 


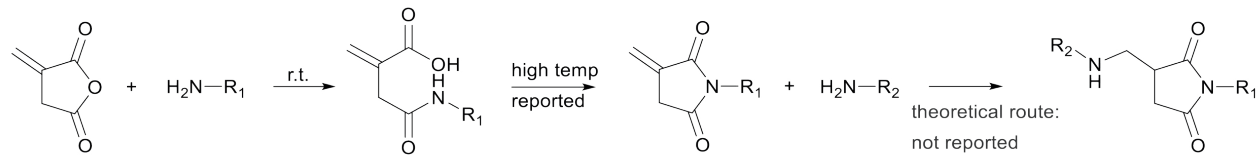

Scheme 4-8. Schematic overview of the reaction of itaconic anhydride with a primary amine (left), which after thermal treatment yields itaconimides (middle), with the unsaturation still present. Although several Michael addition reactions on this double bond have been described, to date no aza-Michael addition reaction has been described.

\section{Aza-Michael addition on polyitaconate}

Itaconic acid has been used plentiful in direct polycondensation with diols, to yield polyesters with the unsaturated bond exo on the main chain. ${ }^{45}$ This unsaturation can undergo the same aza-Michael addition reactions as previously described, and therefore the (co-)polyester can be modified with secondary amines such as diethylamine (Scheme 4-9, top). ${ }^{26,46-48}$ However, when using primary amines, the cascade cyclization reaction can take place, with part of the polymer chain as the leaving group, effectively leading to chain scission (Scheme 4-9, bottom). ${ }^{25}$ This chain scission reaction proved to be a useful tool for reactive degradation of itaconic acid based polyesters. ${ }^{25,47}$

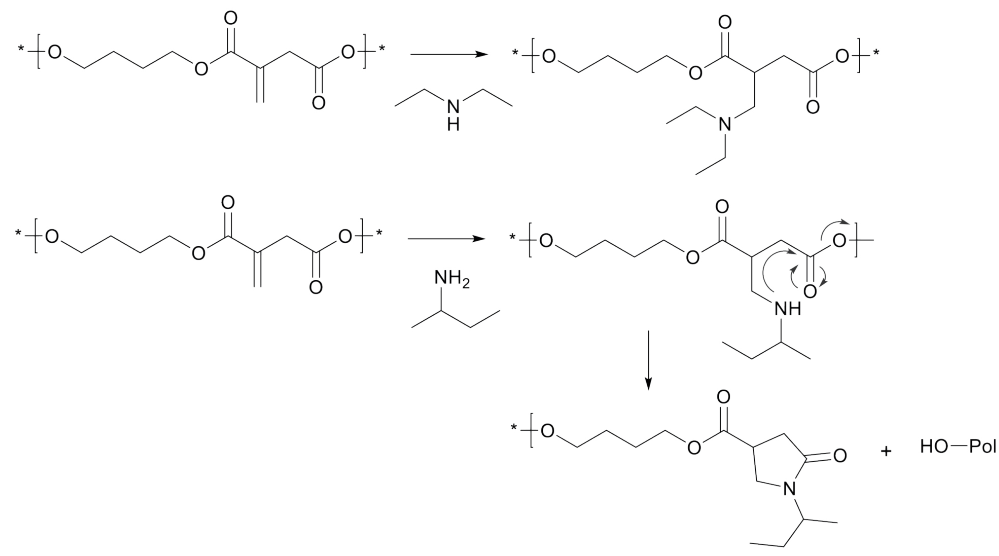

Scheme 4-9. Schematic overview on aza-Michael addition on poly(butylene-itaconate). Top: Modification of exopendant unsaturation with a secondary amine. Bottom: Modification of exo-pendant unsaturation with a primary amine, which is followed by the cyclization step leading to the pyrrolidone ring, and simultaneous chain-scission of the polymer backbone. Reaction arrows are added for clarity. 


\section{Aza-Michael addition on fumaric acid and maleic acid}

Fumaric acid and maleic acid are structural isomers, and it is well-known that fumaric acid can be acid-catalyzed towards maleic acid. ${ }^{49}$ However, similarly to itaconate and mesaconate, this isomerization can also be base-catalyzed by the same amine used for the aza-Michael addition reaction. Such isomerization can happen during polymerization, leading to a change in composition of monomers, and therefore to a change of the desired polymer properties. ${ }^{50}$ In contrary to mesaconate, both fumarate and maleate act as aza-Michael acceptor, though kinetic studies show that maleate react much faster than fumarate (Scheme 4-10), which is likely caused by the higher thermodynamic stability of fumarate. ${ }^{51}$ The combined effect of the presence of two EWGs on the $\beta$ position in respect to the unsaturated bond, and thermodynamically less stable maleate isomer, ensures a high reactivity towards the aza-Michael addition reaction on maleates. The reaction can be performed solvent-free, catalyst-free, and at room temperature, showing the potential of this green transformation. ${ }^{52}$ Some research has been reported on the reversibility of the aza-Michael addition reaction to maleates, and it was shown that the reversible aza-Michael addition only occurred after prolonged heating at $85{ }^{\circ} \mathrm{C}$ when using secondary amines. ${ }^{24}$ In other words, we have found no evidence in literature that the reverse aza-Michael addition proceeds when making use of primary amines and maleates. Furthermore, polymers with varying ratios of fumarate and maleate in the backbone were modified with various primary amines at room temperature, and solely the maleate proved reactive towards aza-Michael addition reaction. ${ }^{53}$

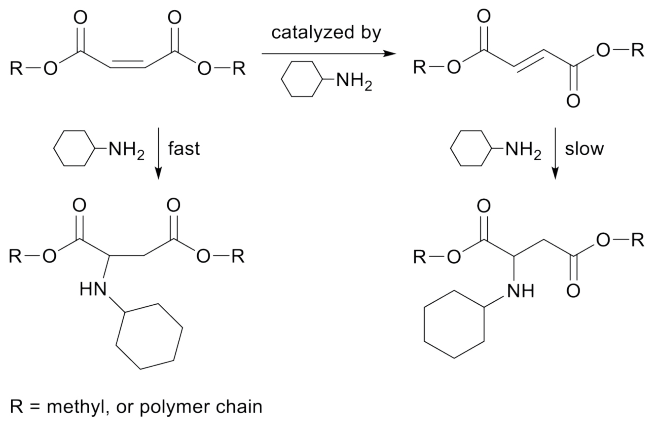

Scheme 4-10. Schematic overview of the reactions between dimethyl maleate and cyclohexylamine. Both the azaMichael addition (left) as the isomerization towards dimethyl fumarate (top) occur fast. The aza-Michael addition to dimethyl fumarate (right) occurs at a lower rate.

Modification of the maleate monomer with an amine, or diamine could lead to interesting new multi-functional monomers which in turn can be used in various polymerization reactions. ${ }^{54}$ However, there are only a very few publications reporting on this transformation for use in polymer synthesis. Similarly, modification of maleate and fumarate containing polymers to achieve desired polymer properties are very limited. Since it was shown that these reactions can be fast, selective, clean, and high yielding, there still seems to be a great unused potential for maleates and fumarates for the synthesis of biobased polymers with enhanced functionality. 
Similar to itaconic anhydride, maleic anhydride shows no reactivity towards the azaMichael addition reaction because the anhydride reacts much faster with amines. Interestingly though, unlike for itaconimides, the imide of maleate (maleimide, Scheme $4-11$, left) is reported to be susceptible towards aza-Michael addition reactions. By reacting 2 equivalents of maleic anhydride with a diamine structure, a bis-maleimide can be obtained, which can be subsequently polymerized with diamines via the aza-Michael addition reaction (Scheme 4-11). ${ }^{55}$ Even though commercially successful polymers (Kerimid $601 \AA)^{56}$ have been prepared this way, this aza-Michael addition reaction is mostly only selective for aromatic amines, and not for aliphatic amines ${ }^{57}$, thereby potentially limiting the clean and selective transformation since aza-Michael reactions with aromatic amines require harsh reaction conditions, and are rarely renewable.

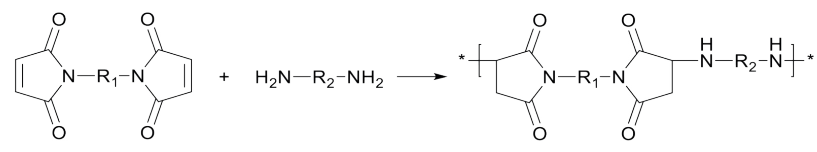

Scheme 4-11. Aza-Michael addition of a di-maleimide with a diamine generating a polyimide.

\section{Recent developments}

\section{Aza-Michael addition on aconitic acid}

Aconitic acid, which is mainly obtained in the trans-configuration from sugar cane molasses $^{58}$, is a very interesting unsaturated ester from biomass due to the tri-carboxylic acid functionality. Both cis- and trans-isomers have seen some use as monomer in polycondensation reactions. For example, mainly cis-aconitic acid is used for its hydrolytic capacity in polyamides, leading to water-soluble polymers which could be hydrolyzed at low $\mathrm{pH}^{59,60}$ In contrast, trans-aconitic acid is more stable as can be deduced by the lower hydrolysis rate ${ }^{61}$ and has successfully been used in the synthesis of hyperbranched polymers. ${ }^{62}$ Despite the better stability of trans-aconitic acid compared to cis-aconitic acid, thermal decarboxylation readily occurs from $105^{\circ} \mathrm{C}^{63,64}$ and higher, generating itaconic acid (Scheme 4-5), thereby limiting its use in high temperature applications. Considering the thermal instability, aconitic acid is an excellent candidate for the cascade aza-Michael addition-cyclization reaction to form the thermally stable pyrrolidone ring (Scheme 4-12). Interestingly this $\mathrm{N}$-substituted-pyrrolidone obtained from aconitic acid has two carboxylic acid groups on the ring, instead of one as is obtained from itaconic acid. 


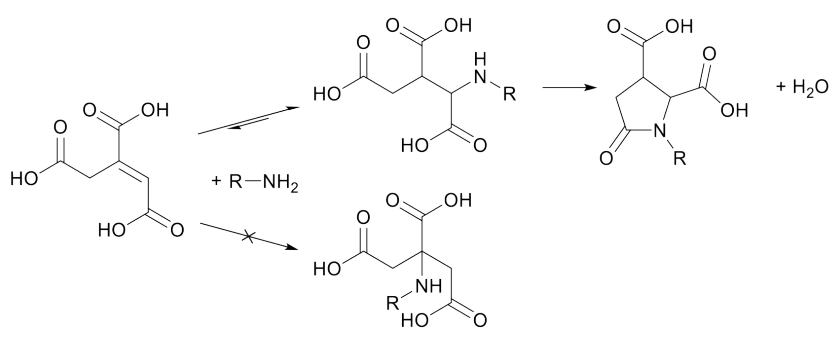

Scheme 4-12. Schematic overview of the aza-Michael addition reaction between aconitic acid and an amine, followed by a cyclization step to form a $\mathrm{N}$-substituted-pyrrolidone ring with 2 carboxylic acid groups on the ring.

To the best of our knowledge the aza-Michael addition between an amine and transtrimethyl aconitate, followed by the cascade cyclization step, has been reported only four times. ${ }^{28,65-67}$ All reported amines were primary mono-amines (e.g. benzylamine), to yield a $\mathrm{N}$-substituted mono-pyrrolidone-dimethylcarboxylate, and found potential use as surfactant. ${ }^{28}$ The reported synthesis methods were in methanol as solvent, at low temperatures (ranging from ambient temperatures up to $40{ }^{\circ} \mathrm{C}$ ), and reported moderate to high (43-85\%) isolated yields after column chromatography. Since the potential of aconitic acid to generate multi-functional carboxylic acids is high, we studied this reaction in more detail with benzylamine, and para-xylylenediamine as model compounds (Scheme 4-13). Details on the experimental methods and analysis are provided in the SI.

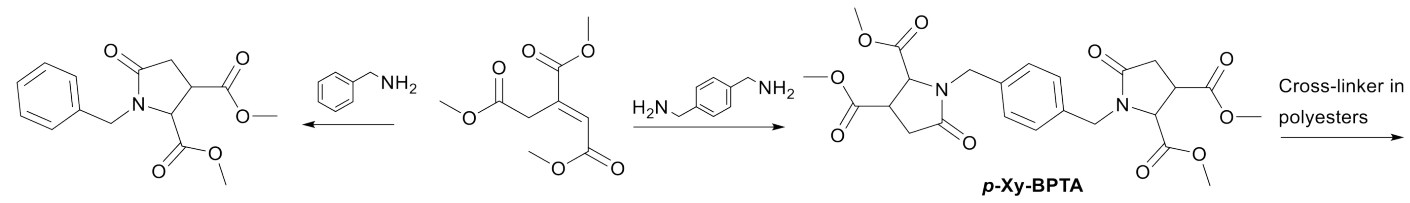

Scheme 4-13. Schematic overview of model aza-Michael addition reaction on trans-trimethyl aconitate as presented in this work.

Upon addition of benzylamine or para-xylylenediamine to a small excess of transtrimethyl aconitate (Scheme 4-13) in methanol at room temperature, the colorless solution turned yellow immediately. Figure 4-1, bottom, shows the ${ }^{1} \mathrm{H}-\mathrm{NMR}$ spectrum of trans-trimethyl aconitate, with the protons of the double bond (5) at $6.9 \mathrm{ppm} .{ }^{1} \mathrm{H}-\mathrm{NMR}$ was immediately performed (Figure 4-1, top) after addition of a $0.95: 1$ molar equivalent of para-xylylenediamine (Figure 4-1, middle) and it was observed that resonance 5 has decreased according to the molar equivalent of diamine added. These findings indicating that the aza-Michael addition reaction has already fully occurred. Note, the ${ }^{1} \mathrm{H}-\mathrm{NMR}-$ spectrum of the expected aza-Michael adduct (Figure 4-1, top) is missing signals for protons 6 and 7 , which likely underwent deuterium-exchange due to their slightly acidic nature, which is supported by the rise in the $\mathrm{H}_{2} \mathrm{O}$ signal. LC-MS studies indicated the presence of minor impurities, which, based on their mass, were identified as products where amide formation occurred between the amine and one of the methyl-esters of trans-trimethyl aconitate. This is considered an undesired side-reaction as it depletes the amines required for the aza-Michael addition. However, this side-reaction could be 
prevented fully by starting the aza-Michael addition reaction at $-20{ }^{\circ} \mathrm{C}$. Overall, these NMR measurements highlight the potential of trans-trimethyl aconitate as aza-Michael acceptor for green transformations of amines, as the aza-Michael addition reaction occurs instantaneously with quantitative conversion, at low temperatures in methanol as green solvent. In other words, we consider the aza-Michael addition with aconitic acid and primary amines a click-reaction, providing a promising route to transform e.g. primary amines or pendant amines, as for example present in chitosan, into trimethylcarboxylate or pyrrolidone based dimethylcarboxylates.

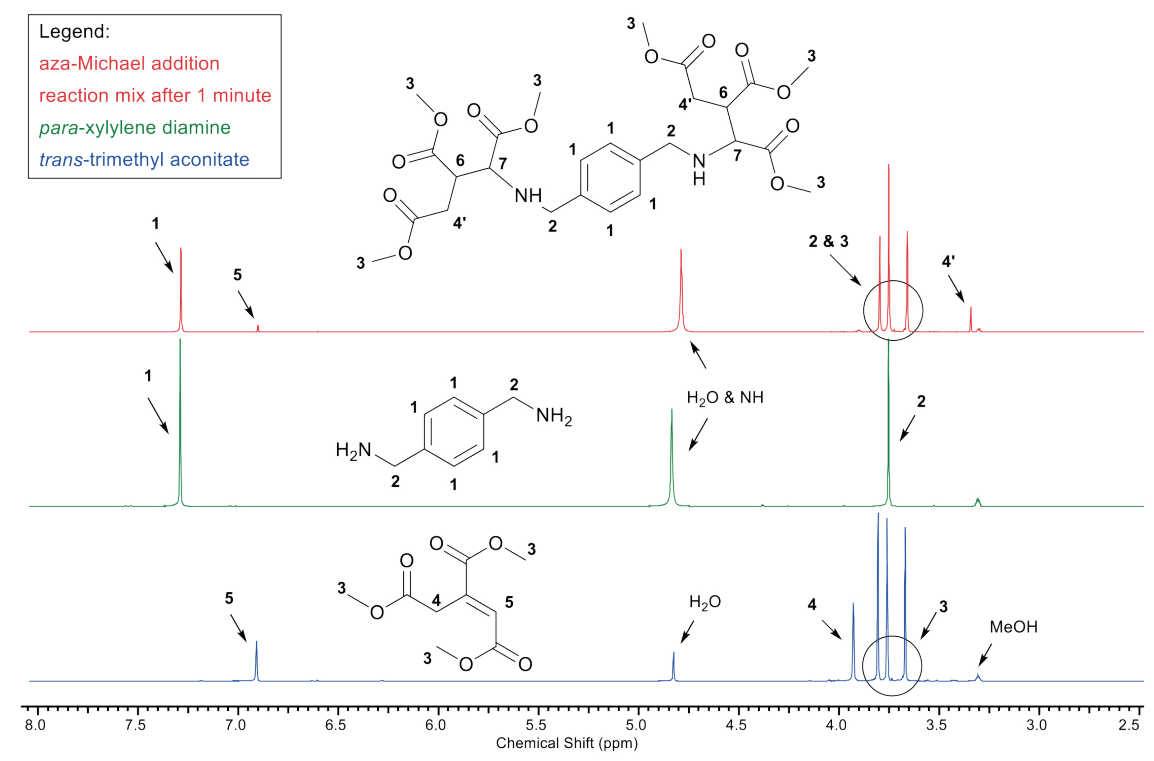

Figure 4-1. ${ }^{1} \mathrm{H}-\mathrm{NMR}$ analysis depicting the aza-Michael addition between trans-trimethyl aconitate (1 equiv.) (blue, bottom) and para-xylylene diamine ( 0.95 equiv.) (green, middle). The reaction mixture (red, top), was measured after 1 minute reaction time at room temperature in deuterated methanol as reaction medium and NMR solvent. The reduction of signal $\mathbf{5}$ indicates the completed aza-Michael addition reaction after 1 minute reaction time.

Previous work reported the cascade cyclization reaction to form the $\mathrm{N}$-substituted pyrrolidone rings in moderate to high yields after prolonged stirring at ambient conditions. ${ }^{66}$ However, both LC-MS and ${ }^{1} \mathrm{H}-\mathrm{NMR}$ analysis show the presence of significant amounts of the ring-opened intermediate, even after stirring for 72 hours at room temperature (details provided in the supplementary information section for this chapter). Instead, the reaction mixture was heated to $125^{\circ} \mathrm{C}$ under reduced pressure for 2 hours (to remove the condensate), which favors the cascade cyclization reaction into the desired pyrrolidone products (LC-MS, SI). The expected products have been isolated via column chromatography as is confirmed by 2D-NMR analysis provided in SI. Even though the purification via column chromatography is not considered green due to the high solvent usage, these findings clearly demonstrate the ease and potential of transtrimethyl aconitate in the aza-Michael cascade reaction. 
Furthermore, in contrast to aconitic acid itself, the isolated para-xylylene-bis-pyrrolidone tetramethylcarboxylate ( $\boldsymbol{p}$-Xy-BPTA, Scheme 4-13) exhibits excellent thermal stability (no weight loss was observed during a 1 hour isotherm at $200{ }^{\circ} \mathrm{C}$ in thermogravimetric analysis, $\mathrm{SI}$ ) which allowed for its use as cross-linker in melt-polycondensation reactions of amorphous BPDA-based polyesters reported in our recent work. ${ }^{30}$ As is depicted in the GPC traces in Figure 4-2, the introduction of 1 or $5 \mathrm{~mol} \%$ of $\boldsymbol{p}$-Xy-BPTA to the monomer mixture containing $\mathrm{C}_{8}$-BPDA-dm and 1,4-butanediol (Scheme 4-14), resulted in rapid branching and cross-linking (details on experimental conditions provided in $\mathrm{SI}$ ). Note, though the GPC traces reflect only the soluble fraction of the synthesized polymers, they clearly indicate a broadening of dispersity by the increase in high molecular weight fractions (Figure 4-2), as is typical for branched and cross-linked polymers. Overall, these findings demonstrate that the aza-Michael cascade reaction can readily be employed to generate multi-functional methylcarboxylates with the thermal stability required for meltpolycondensation reactions. In particular, when using renewable amines, we consider this approach promising for the generation of fully renewable methylcarboxylate based cross-linkers for the use in thermosets or coatings.

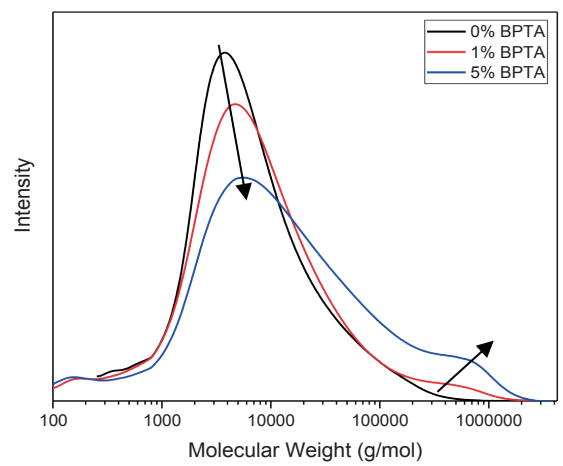

Figure 4-2. GPC traces of the soluble fractions of the obtained polymers with 0,1 , and 5 mol\% of p-Xy-BPTA to generate branched and cross-linked amorphous BPDA-based polyesters. Arrows added to guide the eye. GPC measured in HFIP, and referenced against PMMA standards.

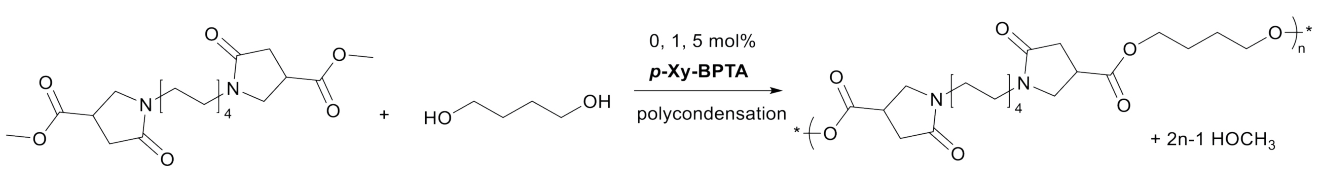

Scheme 4-14. Schematic overview of the melt-polycondensation between $\mathrm{C}_{8}$-BPDA-dm and 1,4-butanediol, with 0, 1, and $5 \mathrm{~mol} \%$ of $\mathbf{p}-\mathbf{X y}$-BPTA cross-linker. 


\section{Future potentials}

\section{Aza-Michael addition on muconic acid}

The last unsaturated biobased di-ester discussed in this work is trans- $\beta$-hydromuconic acid, a derivative from muconic acid, both of which can be polymerized into biobased unsaturated copolyesters. ${ }^{68,69}$ To the best of our knowledge there is no literature precedent on the aza-Michael addition on muconic acid itself, which is likely caused by the conjugation of the double bonds which has a stabilizing effect, and thereby negates the electron negative character of the unsaturated bonds in the $\beta$-position of the EWGs. The conjugation can be negated by partial hydrogenation and isomerization of the double bond to generate $\beta$-hydromuconic acid. However, the unsaturation of $\beta$-hydromuconic acid is not in the $\beta$-position, but in the $\mathrm{\gamma}$-position relative to the EWGs, and is thereby less electron negative than in the previously described unsaturated esters. Nevertheless, several references describe the aza-Michael addition between primary and secondary amines and $\beta$-hydromuconic acid ${ }^{70,71}$, and dimethyl $\beta$-hydromuconate. ${ }^{72}$ Interestingly, here also the cascade cyclization step to form the 5-membered pyrrolidone ring is reported (Scheme 4-15, top). Furthermore, one example is known where trans- $\beta$ hydromuconic acid is reacted with the secondary amine group of several nucleosides, successfully forming the aza-Michael adduct in moderate yields in acetonitrile and in the presence of a base catalyst. (Scheme 4-15, bottom). ${ }^{73}$

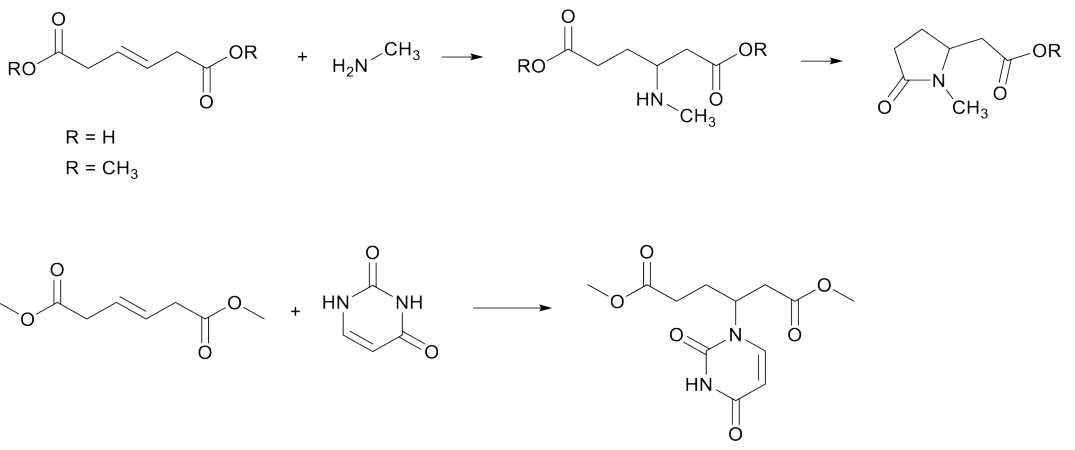

Scheme 4-15. Top: the aza-Michael addition reaction between trans- $\beta$-hydromuconic acid and a primary amine, followed by a cyclization step to obtain $N$-methyl-2-pyrrolidone-ethanoic acid. Bottom: the aza-Michael addition reaction between trans- $\beta$-hydromuconic acid and a secondary amine, leading to the aza-Michael adduct.

Apart from the increased interest of using trans- $\beta$-hydromuconic acid as precursor for adipic acid ${ }^{21,22}$, or for the synthesis of unsaturated polyesters ${ }^{69}$, little recent research interest is shown in this biobased molecule. However, considering the reported formation of the pyrrolidone ring, $\beta$-hydromuconic acid is a very interesting candidate to prepare (bis-)pyrrolidone structures similar to those prepared with itaconates (Scheme 4-7). For example, one can envision the reaction between $\beta$-hydromuconate and a di-amine to form the symmetrical bis-pyrrolidone di-ethanoic acids, structural analogues to the bispyrrolidones obtained from itaconic acid (Scheme 4-16). It is important to note that the reported conditions for the cascade aza-Michael addition-cyclization reaction for trans- $\beta$ - 
hydromuconic acid (e.g. in ethanol at $200{ }^{\circ} \mathrm{C}$ in an autoclave) are harsher compared to e.g. itaconates and aconitates. Such harsh reaction conditions are expected to be required due to the lower expected electronegativity of the unsaturation in $\beta$ hydromuconic acid, but this remains a topic for further investigation. Nevertheless, we consider $\beta$-hydromuconic acid a promising monomer for use in the aza-Michael cascade reaction and expect to see more of this reaction and its products in the future.

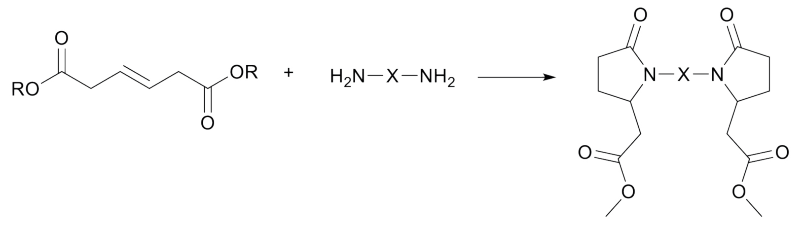

Scheme 4-16. The potential reaction between trans- $\beta$-hydromuconic acid and di-amines leading to bis-pyrrolidone diethanoic acids, a potential structural analogue to itaconic acid based BPDA's.

\section{Conclusion}

In this work, we provided an overview of the aza-Michael addition reaction between amines with several renewable unsaturated dicarboxylic acids (or esters) including itaconic acid, fumaric acid, maleic acid, aconitic acid, and $\beta$-hydromuconic acid. When these monomers bear an ester group in the $y$-position relative to the unsaturation, they prove susceptible to undergo a cascade aza-Michael addition-cyclization reaction to generate a stable 5 -membered $\mathrm{N}$-functionalized pyrrolidone ring, while retaining one carboxylic acid or ester functionality. By careful selection of the amine as Michael donor, $\mathrm{N}$-functional pyrrolidones with a wide range of functional groups can be prepared, which in turn can be used as monomers in melt-polycondensation reactions, allowing for the generation of a wide range of polyesters, poly(ester-amide)s, and polyamides with tunable physio-chemical properties. The state-of-the-art on the aza-Michael cascade reaction is extended in this work with new findings on trimethyl trans-aconitate. The azaMichael addition with primary mono or di-amines can be considered a "click-reaction" that follows the principles of green chemistry: the reaction proceeds quantitatively at room temperature, requires no catalyst, and can be performed in methanol as solvent. Though the ring-closing cascade reaction requires elevated temperatures to reach full conversion, this route transforms the otherwise thermally unstable trimethyl transaconitate into thermally stable bis-pyrrolidone monomer with tetra-methylcarboxylate functionality. Lastly, the potential of this monomer in polycondensation reactions is demonstrated by its successful copolymerization into renewable, branched and crosslinked polyesters.

\section{Conflicts of interest}

There are no conflicts to declare. 


\section{Acknowledgements}

This work was performed under the framework of Chemelot InSciTe and is supported by contributions from the European Regional Development Fund (ERDF) within the framework of OP-Zuid and with contributions from the province of Brabant and Limburg and the Dutch Ministry of Economy

\section{References}

1. Mülhaupt, R. Green Polymer Chemistry and Bio-based Plastics: Dreams and Reality. Macromol. Chem. Phys. 214, 159-174 (2013).

2. Anastas, P. T. \& Kirchhoff, M. M. Origins, Current Status, and Future Challenges of Green Chemistry. Acc. Chem. Res. 35, 686-694 (2002).

3. Kolb, H. C., Finn, M. G. \& Sharpless, K. B. Click Chemistry: Diverse Chemical Function from a Few Good Reactions. Angew. Chemie Int. Ed. 40, 2004-2021 (2001).

4. Rulev, A. Y. Aza-Michael reaction: achievements and prospects. Russ. Chem. Rev. 80, 197-218 (2011).

5. Mather, B. D., Viswanathan, K., Miller, K. M. \& Long, T. E. Michael addition reactions in macromolecular design for emerging technologies. Prog. Polym. Sci. 31, 487-531 (2006).

6. Genest, A., Portinha, D., Fleury, E. \& Ganachaud, F. The aza-Michael reaction as an alternative strategy to generate advanced silicon-based (macro)molecules and materials. Prog. Polym. Sci. 72, 61-110 (2017).

7. Paytash, P. L., Sparrow, E. \& Gathe, J. C. The Reaction of Itaconic Acid with Primary Amines. J. Am. Chem. Soc. 72, 1415-1416 (1950).

8. Wu, Liu, Y., He, Chung \& Goh. Effects of Chemistries of Trifunctional Amines on Mechanisms of Michael Addition Polymerizations with Diacrylates. Macromolecules 37, 6763-6770 (2004).

9. Zou, B. \& Jiang, H.-F. Synthesis of $\beta$-Amino Acids via Catalyst- and Solvent-Free Aza-Michael Reaction. Chinese J. Chem. 26, 1309-1314 (2008).

10. Matveeva, E. V., Petrovskii, P. V. \& Odinets, I. L. Efficient synthesis of racemic $\beta$-aminophosphonates via aza-Michael reaction in water. Tetrahedron Lett. 49, 6129-6133 (2008).

11. Ai, X. et al. An effective aza-Michael addition of aromatic amines to electron-deficient alkenes in alkaline Al2O3. Tetrahedron 66, 5373-5377 (2010).

12. Critchfield, F. E., Funk, G. L. \& Johnson, J. B. Determination of Alpha, Beta-Unsaturated Compouds by Reaction with Morpholine. Anal. Chem. 28, 76-79 (1956).

13. Baruah, R. et al. Recyclable Thermosets Based on Dynamic Amidation and Aza-Michael Addition Chemistry. Macromolecules 49, 7814-7824 (2016).

14. Wilbon, P. A., Chu, F. \& Tang, C. Progress in Renewable Polymers from Natural Terpenes, Terpenoids, and Rosin. Macromol. Rapid Commun. 34, 8-37 (2013).

15. Satoh, K. Controlled/living polymerization of renewable vinyl monomers into bio-based polymers. Polym. J. 47, 527536 (2015).

16. Della Pina, C., Falletta, E. \& Rossi, M. A green approach to chemical building blocks. The case of 3-hydroxypropanoic acid. Green Chem. 13, 1624 (2011).

17. Kanitkar, A., Aita, G. \& Madsen, L. The recovery of polymerization grade aconitic acid from sugarcane molasses. J. Chem. Technol. Biotechnol. 88, 2188-2192 (2013).

18. Mariscal, R., Maireles-Torres, P., Ojeda, M., Sádaba, I. \& López Granados, M. Furfural: a renewable and versatile platform molecule for the synthesis of chemicals and fuels. Energy Environ. Sci. 9, 1144-1189 (2016).

19. Vardon, D. R. et al. cis,cis-Muconic acid: separation and catalysis to bio-adipic acid for nylon-6,6 polymerization. Green Chem. 18, 3397-3413 (2016).

20. Lu, R. et al. Production of Diethyl Terephthalate from Biomass-Derived Muconic Acid. Angew. Chemie Int. Ed. 55, 249253 (2016).

21. Matthiesen, J. E., Carraher, J. M., Vasiliu, M., Dixon, D. A. \& Tessonnier, J.-P. Electrochemical Conversion of Muconic Acid to Biobased Diacid Monomers. ACS Sustain. Chem. Eng. 4, 3575-3585 (2016).

22. Matthiesen, J. E. et al. Electrochemical Conversion of Biologically Produced Muconic Acid: Key Considerations for Scale-Up and Corresponding Technoeconomic Analysis. ACS Sustain. Chem. Eng. 4, 7098-7109 (2016).

23. Farmer, T. J., Macquarrie, D. J., Comerford, J. W., Pellis, A. \& Clark, J. H. Insights into post-polymerisation modification of bio-based unsaturated itaconate and fumarate polyesters via aza-michael addition: Understanding the effects of $\mathrm{C} \square \mathrm{C}$ isomerisation. J. Polym. Sci. Part A Polym. Chem. 56, 1935-1945 (2018).

24. Pellis, A., Hanson, P. A., Comerford, J. W., Clark, J. H. \& Farmer, T. J. Enzymatic synthesis of unsaturated polyesters: functionalization and reversibility of the aza-Michael addition of pendants. Polym. Chem. 10, 843-851 (2019).

25. Farmer, T. J., Comerford, J. W., Pellis, A. \& Robert, T. Post-polymerization modification of bio-based polymers: maximizing the high functionality of polymers derived from biomass. Polym. Int. 67, 775-789 (2018).

26. Chanda, S. \& Ramakrishnan, S. Poly(alkylene itaconate)s - an interesting class of polyesters with periodically located exo-chain double bonds susceptible to Michael addition. Polym. Chem. 6, 2108-2114 (2015).

27. Malferrari, D., Armenise, N., Decesari, S., Galletti, P. \& Tagliavini, E. Surfactants from Itaconic Acid: Physicochemical Properties and Assessment of the Synthetic Strategies. ACS Sustain. Chem. Eng. 3, 1579-1588 (2015). 
28. Okada, Y., Banno, T., Toshima, K. \& Matsumura, S. Synthesis and Properties of Polycarboxylate-type Green Surfactants with S- or N-Linkages. J. Oleo Sci. 58, 519-528 (2009).

29. Noordzij, G. J., Dietz, C. H. J. T., Leoné, N., Wilsens, C. H. R. M. \& Rastogi, S. Small-scale screening of novel biobased monomers: the curious case of 1,3-cyclopentanediol. RSC Adv. 8, 39818-39828 (2018).

30. Noordzij, G. J. et al. The aza-Michael reaction: towards semi-crystalline polymers from renewable itaconic acid and diamines. Polym. Chem. 10, 4049-4058 (2019).

31. Qi, P., Chen, H.-L., Nguyen, H. T. H., Lin, C.-C. \& Miller, S. A. Synthesis of biorenewable and water-degradable polylactam esters from itaconic acid. Green Chem. 18, 4170-4175 (2016).

32. Miller, S. A. \& Qi, P. US2019023839A1 Biorenewable water-degradable polymers and co-polymers. (2019).

33. Ali, M. A., Tateyama, S. \& Kaneko, T. Synthesis of rigid-rod but degradable biopolyamides from itaconic acid with aromatic diamines. Polym. Degrad. Stab. 109, 367-372 (2014).

34. Ali, M. A. et al. Synthesis of High-Performance Biopolyamides Derived from Itaconic Acid and Their Environmental Corrosion. Macromolecules 46, 3719-3725 (2013).

35. Wang, Z. et al. Synthesis of fully bio-based polyamides with tunable properties by employing itaconic acid. Polymer (Guildf). 55, 4846-4856 (2014).

36. He, M., Wang, Z., Wang, R., Zhang, L. \& Jia, Q. Preparation of Bio-Based Polyamide Elastomer by Using Green Plasticizers. Polymers (Basel). 8, 257 (2016).

37. Ali, M. A., Tandon, N., Nag, A. \& Takada, K. Molecular Orientation of Bio-Polyamides After Cryogenic Nanohybridization with Montmorillonites. Arab. J. Sci. Eng. 44, 153-158 (2019).

38. Avny, Y., Saghian, N. \& Zilkha, A. Thermally Stable Polymers Derived from Itaconic Acid. Isr. J. Chem. 10, 949-957 (1972).

39. Gozlan, A., Bernstein, I. \& Zilkha, A. Electrochemical polymerization of dicarboxylic acids-VII. Polymerization of oligomers. Eur. Polym. J. 24, 231-237 (1988).

40. Ayadi, F., Mamzed, S., Portella, C. \& Dole, P. Synthesis of bis(pyrrolidone-4-carboxylic acid)-based polyamides derived from renewable itaconic acid-application as a compatibilizer in biopolymer blends. Polym. J. 45, 766-774 (2013).

41. Wang, R. et al. One-pot synthesis of biodegradable and linear poly(ester amide)s based on renewable resources. J. Appl. Polym. Sci. 133, 43446 (2016).

42. Roy, M., Noordzij, G. J., van den Boomen, Y., Rastogi, S. \& Wilsens, C. H. R. M. Renewable (Bis)pyrrolidone Based Monomers as Components for Thermally Curable and Enzymatically Depolymerizable 2-Oxazoline Thermoset Resins. ACS Sustain. Chem. Eng. 6, 5053-5066 (2018).

43. Roy, M., Wilsens, C. H. R. M., Leoné, N. \& Rastogi, S. Use of Bis(pyrrolidone)-Based Dicarboxylic Acids in Poly(esteramide)-Based Thermosets: Synthesis, Characterization, and Potential Route for Their Chemical Recycling. ACS Sustain. Chem. Eng. 7, 8842-8852 (2019).

44. Ahire, M. M. \& Mhaske, S. B. Synthesis of Succinimide Derivatives by NHC-Catalyzed Stetter Reaction of Aromatic Aldehydes with N -Substituted Itaconimides. ACS Omega 2, 6598-6604 (2017).

45. Robert, T. \& Friebel, S. Itaconic acid - a versatile building block for renewable polyesters with enhanced functionality. Green Chem. 18, 2922-2934 (2016).

46. Guarneri, A. et al. Functionalization of Enzymatically Synthesized Rigid Poly(itaconate)s via Post-Polymerization AzaMichael Addition of Primary Amines. Adv. Synth. Catal. adsc.201900055 (2019). doi:10.1002/adsc.201900055

47. Lv, A., Li, Z.-L., Du, F.-S. \& Li, Z.-C. Synthesis, Functionalization, and Controlled Degradation of High Molecular Weight Polyester from Itaconic Acid via ADMET Polymerization. Macromolecules 47, 7707-7716 (2014).

48. Hoffmann, C., Stuparu, M. C., Daugaard, A. \& Khan, A. Aza-Michael addition reaction: Post-polymerization modification and preparation of PEI/PEG-based polyester hydrogels from enzymatically synthesized reactive polymers. J. Polym. Sci. Part A Polym. Chem. 53, 745-749 (2015).

49. Davies, M. \& Evans, F. P. The isomerization of maleic acid in aqueous solutions. Trans. Faraday Soc. 52, 74 (1956).

50. Jada, S. S. Role of Amines in the Isomerization Reaction of Polycondensed Maleic Anhydride and Diethylene Glycol. J. Macromol. Sci. Part A - Chem. 22, 57-73 (1985).

51. Bláha, M. et al. Solvent-free, catalyst-free aza-Michael addition of cyclohexylamine to diethyl maleate: Reaction mechanism and kinetics. Tetrahedron 74, 58-67 (2018).

52. Bosica, G. \& Debono, A. J. Uncatalyzed, green aza-Michael addition of amines to dimethyl maleate. Tetrahedron 70, $6607-6612$ (2014).

53. Yu, Y., Wei, Z., Leng, X. \& Li, Y. Facile preparation of stereochemistry-controllable biobased poly(butylene maleate- co -butylene fumarate) unsaturated copolyesters: a chemoselective polymer platform for versatile functionalization via azaMichael addition. Polym. Chem. 9, 5426-5441 (2018).

54. Tapaswi, P. K. et al. Synthesis and characterization of fully aliphatic polyimides from an aliphatic dianhydride with piperazine spacer for enhanced solubility, transparency, and low dielectric constant. J. Polym. Sci. Part A Polym. Chem. 52, 2316-2328 (2014).

55. Dolci, E. et al. Maleimides As a Building Block for the Synthesis of High Performance Polymers. Polym. Rev. 56, 512556 (2016).

56. Fullerton, R., Roylance, D., Allred, R. \& Acton, A. Cure analysis of printed wiring boards containing reactive adhesive layers. Polym. Eng. Sci. 28, 372-376 (1988).

57. Gherasim, M. G. \& Zugrăvescu, I. Polydisuccinimides: Polyaddition reactions of aliphatic and aromatic diamines to N,N'bismaleimide. Eur. Polym. J. 14, 985-990 (1978).

58. Montoya, G., Londono, J., Cortes, P. \& Izquierdo, O. Quantitation of trans -Aconitic Acid in Different Stages of the SugarManufacturing Process. J. Agric. Food Chem. 62, 8314-8318 (2014).

59. DuBois Clochard, M.-C., Rankin, S. \& Brocchini, S. Synthesis of soluble polymers for medicine that degrade by intramolecular acid catalysis. Macromol. Rapid Commun. 21, 853-859 (2000).

60. Zloh, M., Dinand, E. \& Brocchini, S. Aconityl-derived polymers for biomedical applications. Modeling study of cis-trans 
isomerisation. Theor. Chem. Accounts Theory, Comput. Model. (Theoretica Chim. Acta) 109, 206-212 (2003).

61. Dinand, E., Zloh, M. \& Brocchini, S. Competitive Reactions During Amine Addition to cis-Aconityl Anhydride. Aust. J. Chem. 55, 467 (2002).

62. Cao, H., Zheng, Y., Zhou, J., Wang, W. \& Pandit, A. A novel hyperbranched polyester made from aconitic acid (B3) and di(ethylene glycol) (A2). Polym. Int. 60, 630-634 (2011).

63. Sepulchre, M.-O. \& Sepulchre, M. Synthesis of unsaturated polyesters from dipotassium salts of cis-aconitic, itaconic and mesaconic acids and 1, 4-dibromobutane. Macromol. Symp. 122, 291-296 (1997).

64. Noordover, B. A. J., Duchateau, R., van Benthem, R. A. T. M., Ming, W. \& Koning, C. E. Enhancing the Functionality of Biobased Polyester Coating Resins through Modification with Citric Acid. Biomacromolecules 8, 3860-3870 (2007).

65. Schäfer, H. J., Witthaut, D. \& Kratschmer, S. Trimethyl Aconitate. in Encyclopedia of Reagents for Organic Synthesis (John Wiley \& Sons, Ltd, 2009). doi:10.1002/047084289X.rn00999

66. Singh, A. K., Das, S. \& Datta, A. A case study on the myth of emission from aliphatic amides. Methods Appl. Fluoresc. 4, 047003 (2016).

67. Joentgen, W. et al. DE10041903A1 New aconitic acid derivatives useful as ultraviolet absorbers, fluorescent dyes, marker dyes, optical brighteners and intermediates for pharmaceutical, veterinary or plant protection agents. (2002).

68. Rorrer, N. A. et al. Renewable Unsaturated Polyesters from Muconic Acid. ACS Sustain. Chem. Eng. 4, 6867-6876 (2016).

69. Yu, Y. et al. High Molecular Weight Unsaturated Copolyesters Derived from Fully Biobased trans - $\beta$-Hydromuconic Acid and Fumaric Acid with 1,4-Butanediol: Synthesis and Thermomechanical Properties. ACS Sustain. Chem. Eng. 7, 6859-6869 (2019).

70. Evans, G. L., Gray, H. W. \& Jacobson, H. W. Synthesis of Ecgoninic Acid and Related Pyrrolidones. J. Am. Chem. Soc. 72, 2727-2728 (1950).

71. Lavagnino, E. R. \& Ryan, C. W. The reaction of trans -3-hexenedioic acid with ethylenediamines. A simple route to the tetrahydro-1H-pyrrole[1,2-d][1,4]diazepine ring system. Org. Prep. Proced. Int. 15, 251-260 (1983).

72. Wu, Y.-H., Corrigan, J. R. \& Feldkamp, R. F. Pyrrolidines. IV. The Investigation of the Synthesis of 1-Methyl-2pyrrolidineethanol. J. Org. Chem. 26, 1531-1533 (1961).

73. Perbost, M., Lucas, M., Chavis, C. \& Imbach, J. L. Synthesis of racemic carboacyclonucleosides derived from butane1, 4-diol and hexane-1, 6-diol. Nucleosides and Nucleotides 11, 1489-1505 (1992). 


\section{Supplementary information for chapter 4}

\section{Materials and Methods}

\section{Materials}

1,4-Butanediol, benzylamine, and Sn(II)2-ethylhexanoate were purchased from Sigma Aldrich. Transtrimethyl aconitate and $p$-xylylene-diamine were purchased from $\mathrm{TCl}$ chemicals. Standard laboratory solvents were obtained from BioSolve. Deuterated solvents were obtained from Buchem BV (Netherlands). $\mathrm{C}_{8}$-BPDA-dm was synthesized from dimethyl itaconate and 1,8-diamino octane according to the synthesis methods described in chapter 5 . The purchased compounds were used directly without further purification, unless otherwise specified.

\section{Analysis methods}

A Grace Reveleris X2 automatic flash column was used for the purification of products. ${ }^{1} \mathrm{H}-\mathrm{NMR}$ and ${ }^{13} \mathrm{C}$ NMR spectra were recorded with a Bruker Ultrashield 300 spectrometer ( $300 \mathrm{MHz}$ magnetic field). Liquid Chromatography - Mass Spectrometry (LC-MS) analysis was performed on a Shimadzu Nexera 2 UHPLC system equipped with a Shimadzu LC-30AD pump, a SPD-M30A photodiode array detector and LCMS-2020 single quadrupole detector. The system was run on MilliQ water and LC-MS grade acetonitrile both modified with $0.1 \%$ formic acid. The following column was used on a Waters XSelect CSH C18 (3.0 $\mathrm{mm} \times 75 \mathrm{~mm}$ with a particle size of $3.5 \mu \mathrm{m}$ ) operating at $30{ }^{\circ} \mathrm{C}$. The method was setup with the following gradient: $5 \%$ acetonitrile in water for $2 \mathrm{~min}$, an increase to $95 \%$ acetonitrile over $6 \mathrm{~min}, 1 \mathrm{~min}$ at $95 \%$ followed by flushing of the column back to $5 \%$ acetonitrile. All samples were prepared by dissolving 1-3 $\mathrm{mg} / \mathrm{ml}$ of material in methanol or acetonitrile and filtering over $0.2 \mu \mathrm{m}$ PFTE filter. NMR-samples were prepared by dissolving ca. $10 \mathrm{mg}$ of sample in $0.5 \mathrm{~mL}$ deuterated solvent, including dimethyl sulfoxide (DMSO- $\left.d_{6}\right)$, deuterated chloroform $\left(\mathrm{CDCl}_{3}\right)$, and deuterated trifluoroacetic acid $(d-\mathrm{TFA})$. All spectra were referenced against tetramethylsilane (TMS), or residual solvent peak from the deuterated solvent. Molecular weight $\left(M_{\mathrm{n}}, M_{\mathrm{w}}\right)$ and dispersity $(\Theta)$ of the polymers were calculated after gel permeation chromatography (GPC). HFIP-GPC was measured on a PSS SECcurity GPC system using Agilent 1260 Infinity instrument technology. The GPC was equipped with a PFG Combination pre-column and two PFG Combination micro-columns. Distilled HFIP containing $0.019 \%$ sodium trifluoroacetate was used as mobile phase at $40{ }^{\circ} \mathrm{C}$, with a $0.3 \mathrm{~mL} / \mathrm{min}$ flow rate. $\mathrm{CHCl}_{3}-\mathrm{GPC}$ was measured on a Prominence-I LC-2030 equipped with a Shodex GPC KF-805L column. Analytical grade $\mathrm{CHCl}_{3}$ was used as mobile phase at $40{ }^{\circ} \mathrm{C}$, with a flowrate of $1 \mathrm{~mL} / \mathrm{min}$. GPC samples were prepared by dissolving ca. $5 \mathrm{mg}$ of polymer in 1.5 $\mathrm{mL}$ of solvent overnight under constant shaking, the samples were filtered over a $0.2 \mu \mathrm{m}$ PTFE syringe filter prior to injection. Thermal stability of compounds were performed via thermogravimetric analysis (TGA) using a TA Instruments Q500. Experiments were performed under a nitrogen atmosphere with a heating rate of $10^{\circ} \mathrm{C} / \mathrm{min}$.

\section{Synthesis methods}

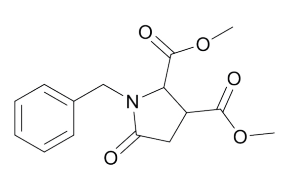

\section{Synthesis of $N$-benzyl-pyrrolidone-2,3-dicarboxylate}

At $-20{ }^{\circ} \mathrm{C}$, a solution of benzylamine $(1.018 \mathrm{~g}, 9.5 \mathrm{mmol})$ in $2.5 \mathrm{~mL}$ methanol was added dropwise to a stirred solution of trans-trimethyl-aconitate $(2.162 \mathrm{~g}, 10 \mathrm{mmol})$ in $2.5 \mathrm{~mL}$ methanol. The solution turned yellow immediately. The solution was stirred overnight, whilst warming up to room temperature, and the color changed to dark brown. Subsequently the solution was heated to $125^{\circ} \mathrm{C}$ for 2 hours, and finally one hour of vacuum was applied at $125^{\circ} \mathrm{C}$ to yield a viscous brown oil. The product was purified via column chromatography (ethyl acetate : heptane) with a 
gradient from 1:2 to $1: 1$. The product is isolated as orange oil, which crystallized over time $(\mathrm{RF} 0.2,1.81 \mathrm{~g}$, $65 \%$ yield). ${ }^{1} \mathrm{H}-\mathrm{NMR}\left(\mathrm{CDCl}_{3}, 300 \mathrm{MHz}\right): \delta 7.41-7.17(\mathrm{~m}, 5 \mathrm{H}), 5.05-4.91(\mathrm{~m}, 0.98 \mathrm{H}), 4.33-4.17(\mathrm{~m}, 1 \mathrm{H}), 4.15-$ $3.93(\mathrm{~m}, 1.03 \mathrm{H}), 3.74-3.62(\mathrm{~m}, 5.91 \mathrm{H}), 3.47-3.24(\mathrm{~m}, 1.04 \mathrm{H}), 3.11-2.58(\mathrm{~m}, 2.02 \mathrm{H}) .{ }^{13} \mathrm{C}-\mathrm{NMR}\left(\mathrm{CDCl}_{3}, 300\right.$ $\mathrm{MHz}): \delta 173.0,172.6,171.8,170.7,170.4,169.9,135.3,135.2,128.8,128.7,128.6,128.4,128.0,127.9$, $61.0,60.5,52.8,52.5,45.7,40.8,40.2,33.0,32.5$.

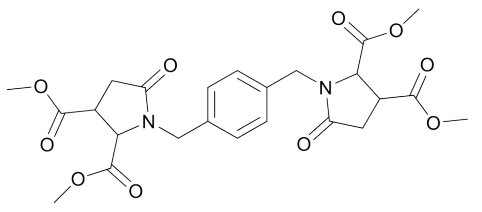

\section{Synthesis of $N, N^{\prime}$-para-xylylene-bis-pyrrolidone-2,3-dicarboxylate $(p-X y-B P T A)$}

At $-20{ }^{\circ} \mathrm{C}$, a solution of para-xylylenediamine $(0.647 \mathrm{~g}, 4.75 \mathrm{mmol})$ in $2.5 \mathrm{~mL}$ methanol was added dropwise to a stirred solution of transtrimethyl-aconitate $(2.162 \mathrm{~g}, 10 \mathrm{mmol})$ in $2.5 \mathrm{~mL}$ methanol. The solution turned yellow immediately. The solution was stirred overnight, whilst warming up to room temperature, and the color changed to dark brown. Subsequently the solution was heated to $125{ }^{\circ} \mathrm{C}$ for 2 hours, and finally 2 hours of vacuum was applied at $125^{\circ} \mathrm{C}$ to yield a viscous brown oil. The product was purified via column chromatography (methanol : chloroform) with a gradient from $2 \%$ to $10 \%$ methanol. The product is isolated as green transparent solid, ( $\mathrm{R}_{\mathrm{F}} 0.15,1.33 \mathrm{~g}, 56 \%$ yield). ${ }^{1} \mathrm{H}-\mathrm{NMR}\left(\mathrm{CDCl}_{3}, 300 \mathrm{MHz}\right)$ : б 7.15-7.07 (m, 3.89H), 4.98-4.83 (m, 2H), 4.25-4.08 (m, 2.02H), 4.04-3.81 (m, 2.11H), 3.67-3.53 (m, $12.01 \mathrm{H}), 3.41-3.17(\mathrm{~m}, 2.13 \mathrm{H}), 3.01-2.51(\mathrm{~m}, 4.14 \mathrm{H}) .{ }^{13} \mathrm{C}-\mathrm{NMR}\left(\mathrm{CDCl}_{3}, 300 \mathrm{MHz}\right): \delta 172.6,171.8,170.6$, $169.8,134.9,128.9,128.7,128.6,61.0,60.5,52.8,52.5,45.3,40.7,40.2,33.0,32.5$.

\section{Polymerization of $\mathrm{C}_{8}$-BPDA-dm, 1,4-butanediol, and p-Xy-BPTA}

Polyesters of $\mathrm{C}_{8}$-BPDA-dm and 1,4-butanediol with varying amounts of $p$-Xy-BPTA have been prepared. Upon addition of $p$-Xy-BPTA, a portion of $\mathrm{C}_{8}$-BPDA-dm was left out to ensure a stable acid/alcohol ratio. The exact amounts weighed in can be found in Table S4 - 1. Exemplified is the polymerization with $5 \mathrm{~mol} \%$ BPTA: $C_{8}$-BPDA-dm (1.42 g, $\left.3.6 \mathrm{mmol}\right), 1,4$-butanediol (0.73 g, $\left.8.1 \mathrm{mmol}\right), p$-Xy-BPTA (0.052 g, $\left.0.1 \mathrm{mmol}\right)$ were loaded in a $100 \mathrm{~mL} 3$-neck round-bottom flask, equipped with a $\mathrm{N}_{2} /$ vacuum inlet, vigreux column, and a heavy duty mechanical stirrer. The reaction mixture was purged 3 times with a vacuum $/ \mathrm{N}_{2}$ cycle. The reaction mixture was slowly heated under light $\mathrm{N}_{2}$ flow to $120{ }^{\circ} \mathrm{C}$, after which the catalyst $\mathrm{Sn}(\mathrm{II}) 2$ ethylhexanoate (16.7 mg, $0.041 \mathrm{mmol}$, in $1 \mathrm{~mL}$ anhydrous DMF) was added. For the oligomerization step, the reaction mixture was gradually heated to $180{ }^{\circ} \mathrm{C}$ where it was kept isothermal for 2 hours to allow for the generation and distillation of the condensate and DMF. Next, vacuum was applied for $4-8$ hours to allow build-up of molecular weight. The final polymers were obtained as a light orange sticky viscous solids and were used without further purification.

Table S4 - 1. Overview of added monomer ratio in the polymerization of C8-BPDA-dm, 1,4-butanediol, and $\boldsymbol{p}$-Xy-BPTA.

\begin{tabular}{c|c|c|c|c|c|c|} 
Mol \% p-Xy-BPTA & \multicolumn{2}{c}{$\mathbf{1 , 4 - b u t a n e d i o l ~}$} & \multicolumn{2}{c}{$\mathbf{C}_{8}$-BPDA-dm } & \multicolumn{2}{c}{$\boldsymbol{p}$-Xy-BPTA } \\
\hline \multirow{3}{*}{$\begin{array}{c}\text { No cross-linker } \\
1 \mathrm{~mol} \%\end{array}$} & $\mathbf{g}$ & $\mathbf{m o l}$ & $\mathbf{g}$ & $\mathbf{m o l}$ & $\mathbf{g}$ & $\mathbf{m o l}$ \\
\cline { 2 - 7 } & 0.73 & 8.14 & 1.5 & 3.78 & - & - \\
\cline { 2 - 7 } $5 \mathrm{~mol} \%$ & 0.73 & 8.14 & 1.48 & 3.75 & 0.010 & 0.02 \\
\cline { 2 - 7 } & 0.73 & 8.14 & 1.42 & 3.59 & 0.052 & 0.10 \\
\cline { 2 - 7 }
\end{tabular}




\section{ELSD analysis of $p$-Xylylene-BPTA monomer synthesis}

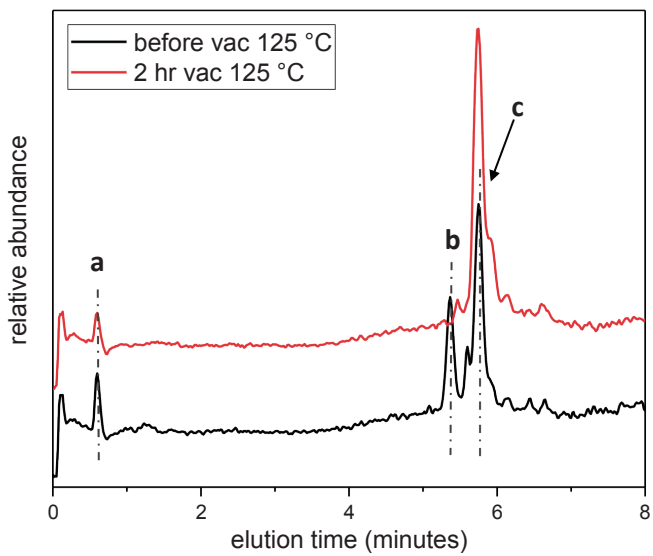

Figure S4 - 1. ELSD traces of the synthesis of $\boldsymbol{p}$-Xy-BPTA. Bottom (black): $2 \mathrm{hr} @ 125{ }^{\circ} \mathrm{C}$, top (red): $2 \mathrm{hr}$ @ $125{ }^{\circ} \mathrm{C}$ under vacuum. A: $p$-xylylene-diamine reacted once with trimethyl-aconitate. B: intermediate before cyclization reaction. C: product. It can be observed how peak B has decreased significantly after the vacuumstep, and nearly only product peak $\mathbf{C}$ is present. This resulting reaction mixture after the vacuumstep was subsequently purified via column chromatography to isolate the product $\boldsymbol{p}$-Xy-BPTA.

\section{D-NMR analysis of monomers}

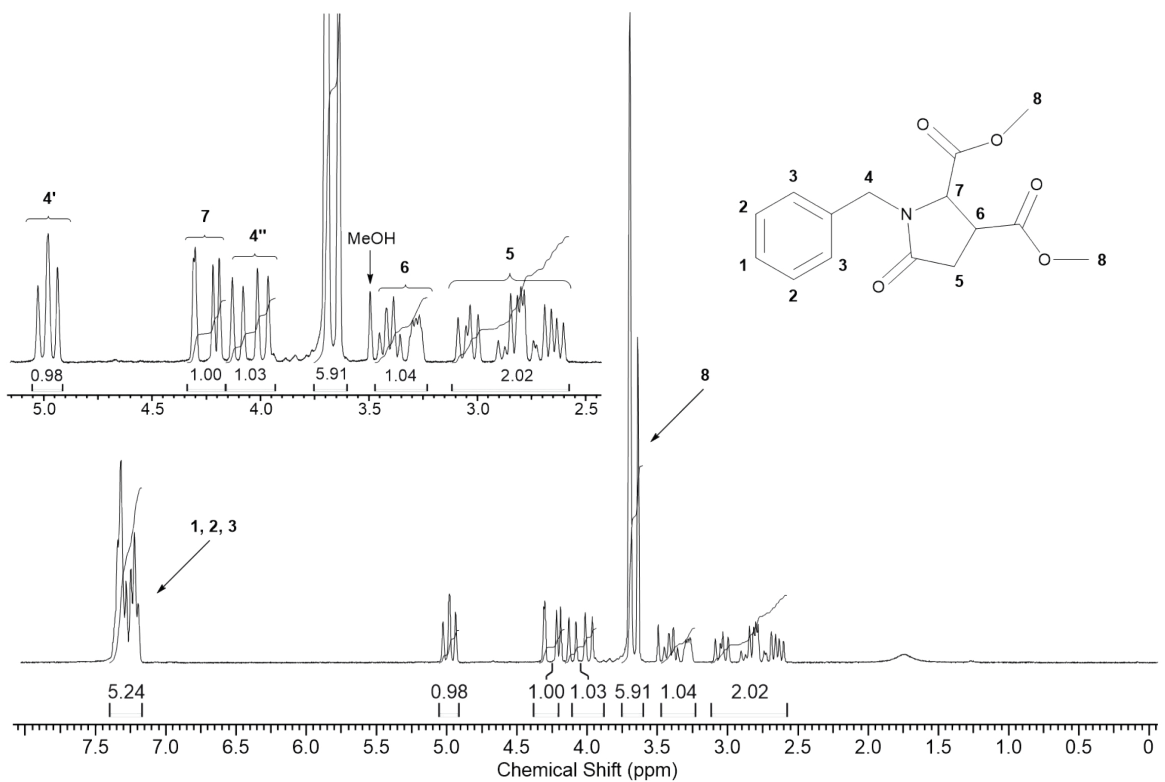

Figure S4 - 2. ${ }^{1} \mathrm{H}-\mathrm{NMR}$ spectrum of $\mathrm{N}$-benzyl-2,3-pyrrolidone-dicarboxylate. 


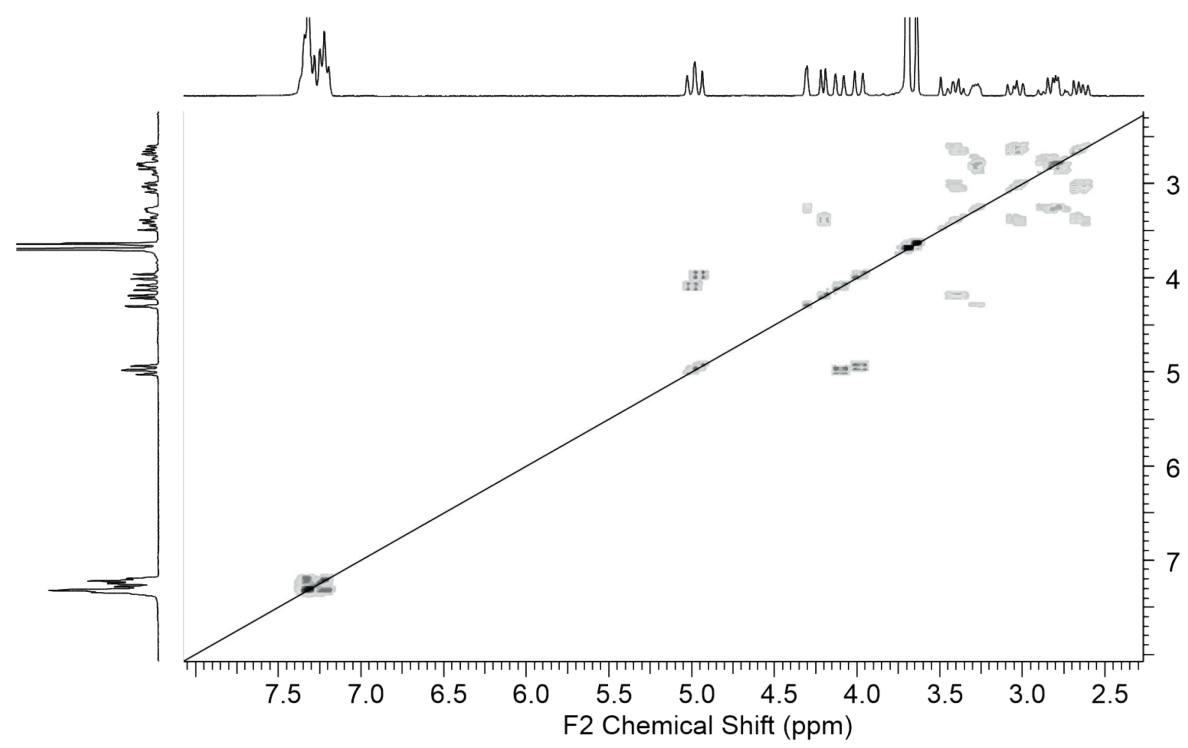

Figure S4 - 3. COSY $\left({ }^{1} \mathrm{H}-{ }^{1} \mathrm{H}\right) \mathrm{NMR}$ spectrum of $\mathrm{N}$-benzyl-2,3-pyrrolidone-dicarboxylate.

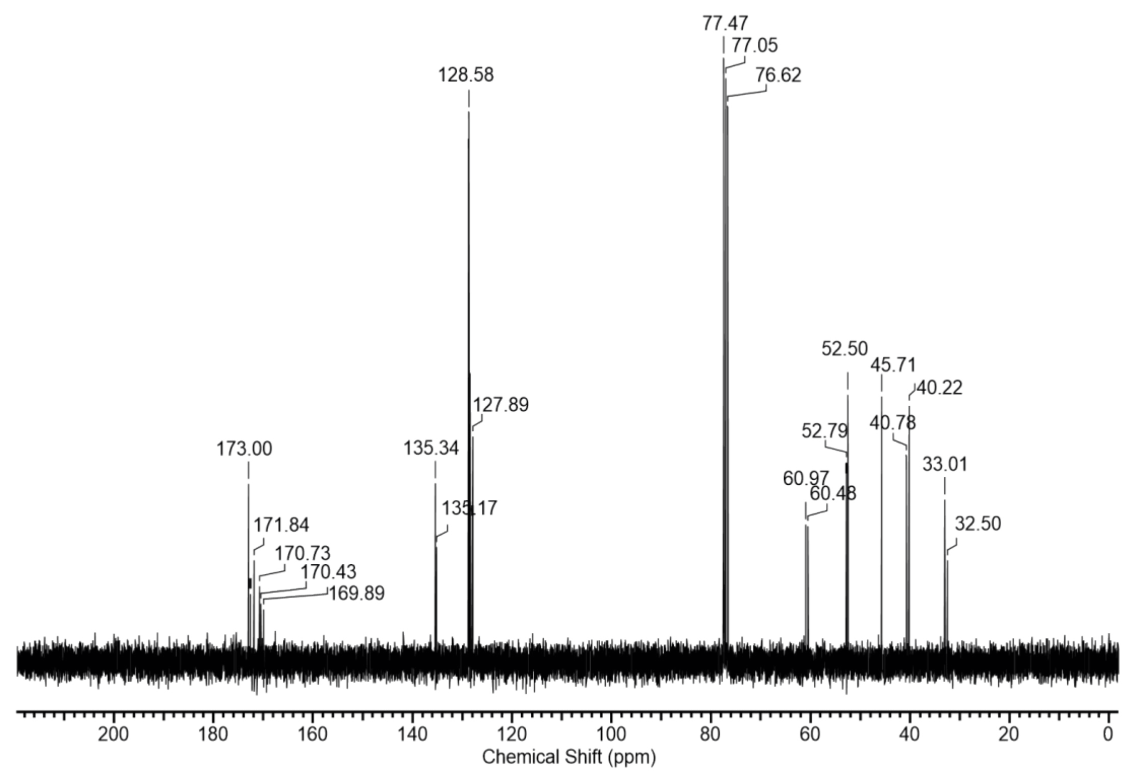

Figure S4 - 4. ${ }^{13} \mathrm{C}-\mathrm{NMR}$ spectrum of $\mathrm{N}$-benzyl-2,3-pyrrolidone-dicarboxylate. 


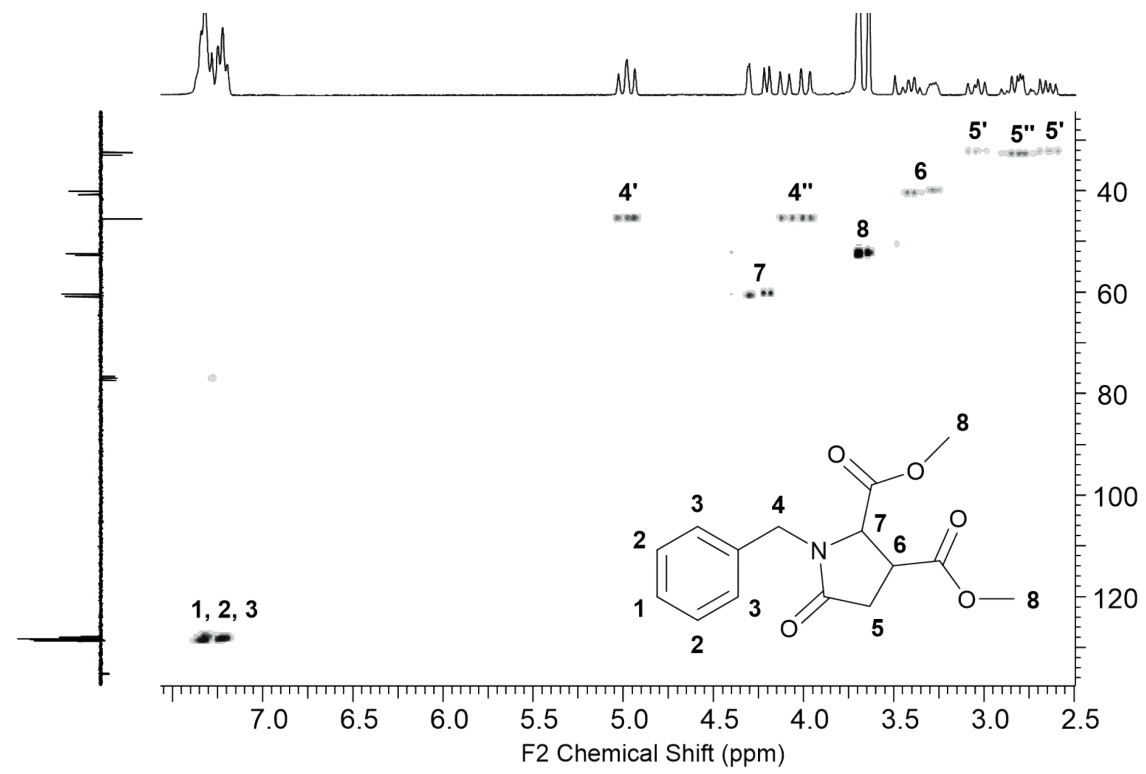

Figure S4 - 5. HSQC ( $\left.{ }^{1} \mathrm{H}-{ }^{13} \mathrm{C}-\mathrm{APT}\right) \mathrm{NMR}$ spectrum of $\mathrm{N}$-benzyl-2,3-pyrrolidone-dicarboxylate.

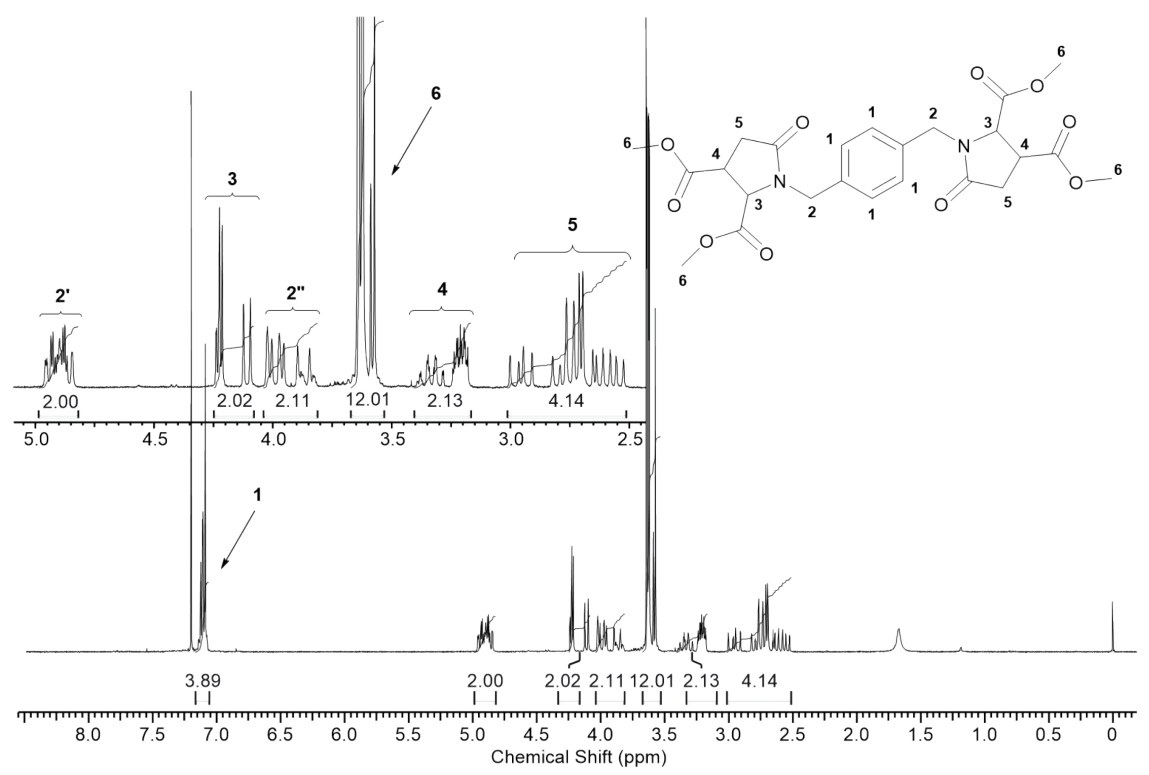

Figure S4 - 6. ${ }^{1} \mathrm{H}-\mathrm{NMR}$ spectrum of $N, N^{\prime}$-para-xylylene-bis-2,3-pyrrolidone-carboxylate. 


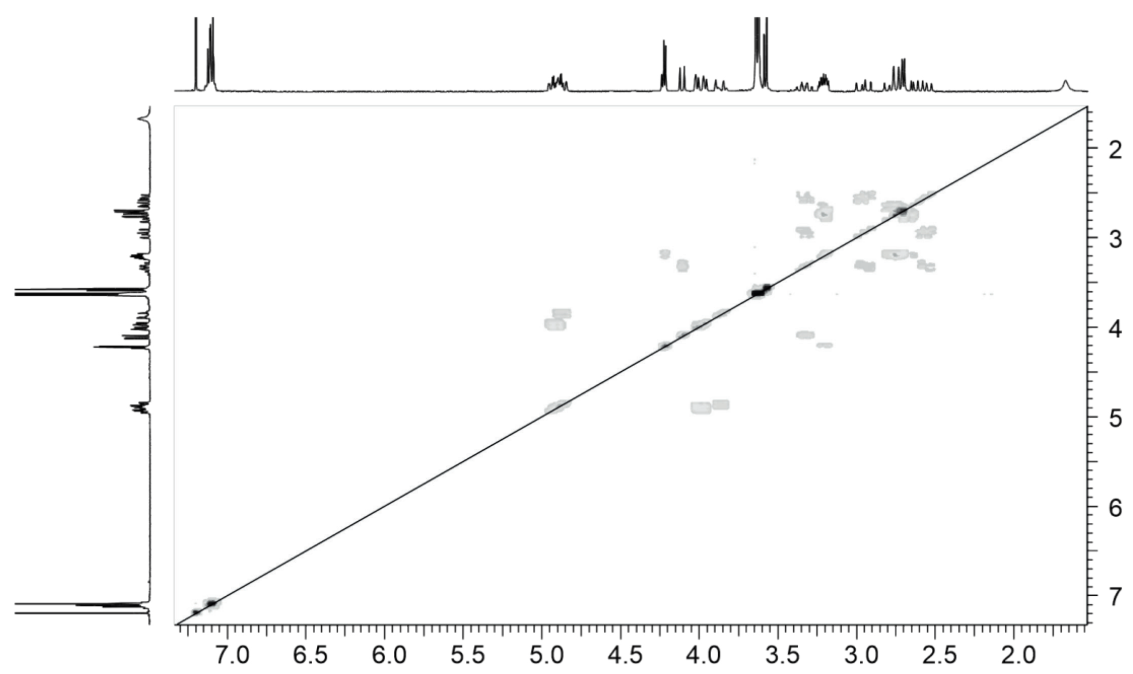

Figure $\mathrm{S} 4$ - 7. COSY $\left({ }^{1} \mathrm{H}-{ }^{1} \mathrm{H}\right)$ NMR spectrum of $N, N$-para-xylylene-bis-2,3-pyrrolidone-carboxylate.

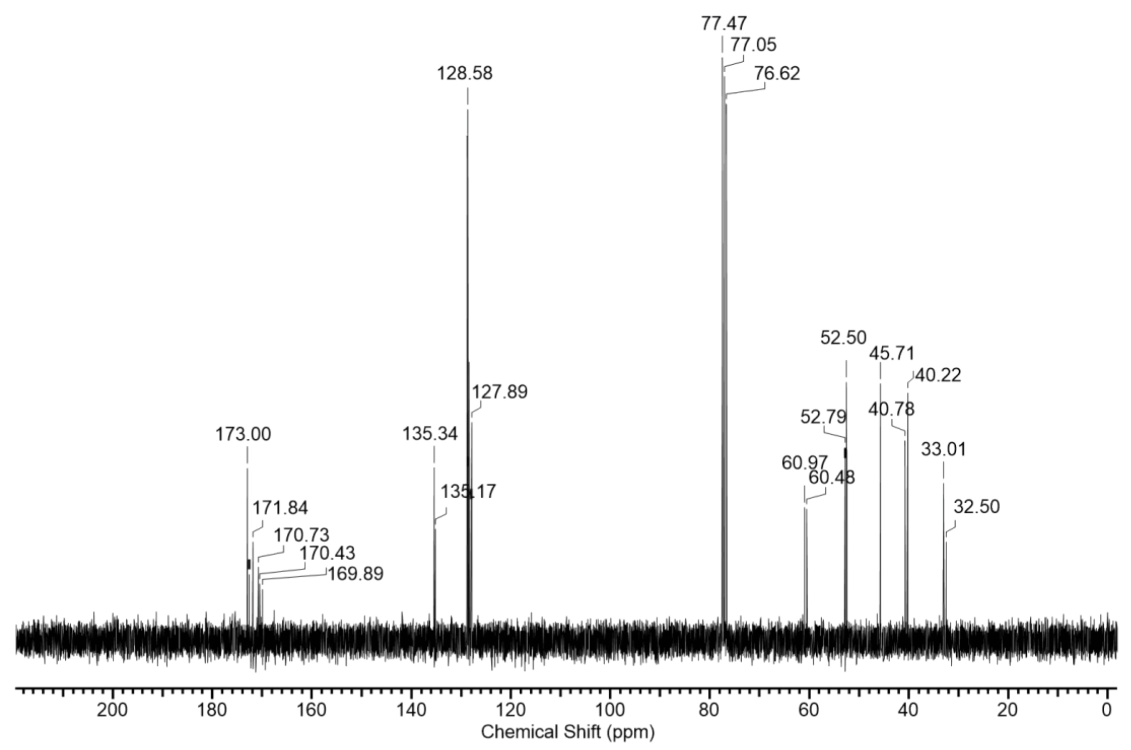

Figure $\mathrm{S} 4-8 .{ }^{13} \mathrm{C}-\mathrm{NMR}$ spectrum of $N, N$-para-xylylene-bis-2,3-pyrrolidone-carboxylate. 


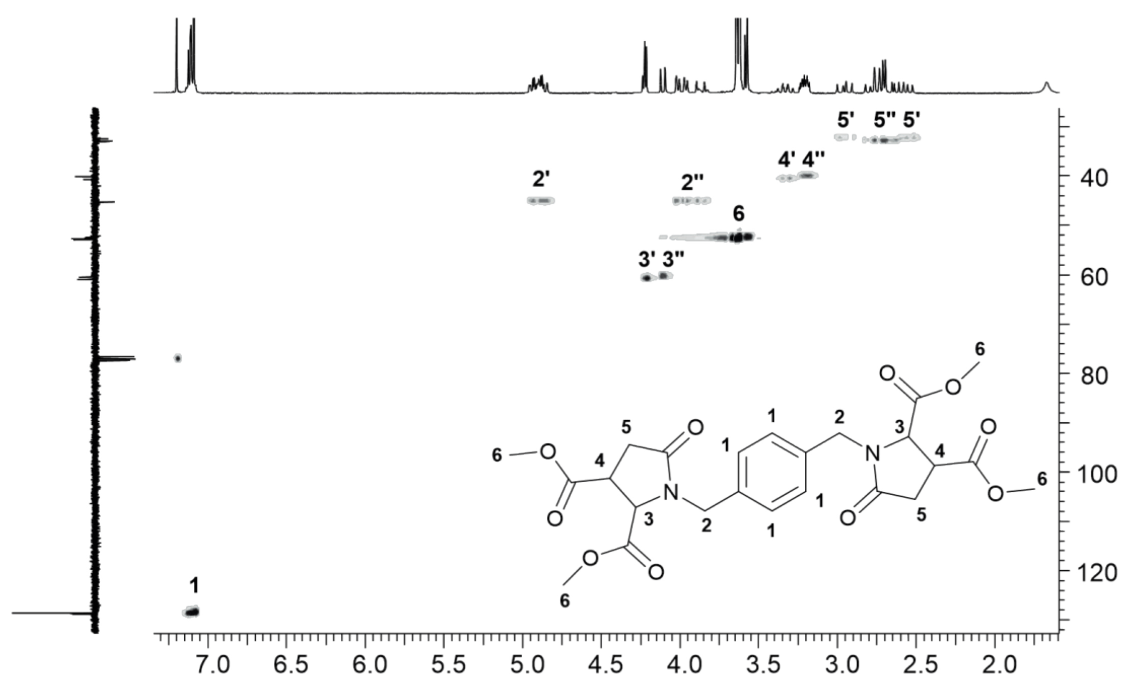

Figure S4 - 9. HSQC ( $\left.{ }^{1} \mathrm{H}^{13} \mathrm{C}-\mathrm{APT}\right) \mathrm{NMR}$ spectrum of $N, N^{\prime}$-para-xylylene-bis-2,3-pyrrolidone-carboxylate.

\section{TGA analysis of para-Xylylene-BPTA}

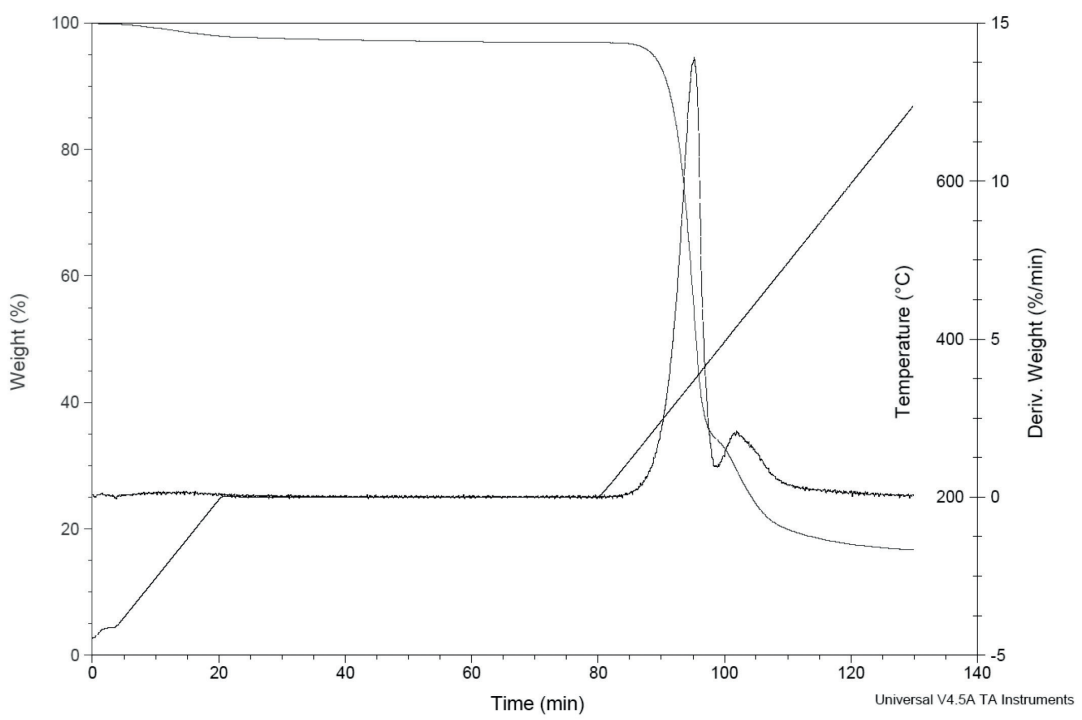

Figure S4 - 10. TGA analysis of $\boldsymbol{p}$-Xy-BPTA monomer, with a one hour isothermal step at $200{ }^{\circ} \mathrm{C}$ showing no weight loss, indicating $p$-Xy-BPTA is stable at this temperatures. Program: $10^{\circ} \mathrm{C} / \mathrm{min}$ to $200{ }^{\circ} \mathrm{C}, 60$ minutes isothermal at $200{ }^{\circ} \mathrm{C}, 10^{\circ} \mathrm{C} / \mathrm{min}$ to $700^{\circ} \mathrm{C}$. 


\section{Chapter 5 The Aza-Michael reaction: Towards semi-crystalline polymers from renewable itaconic acid and diamines}

This chapter is published as:

The Aza-Michael reaction: Towards semi-crystalline polymers from renewable itaconic acid and diamines

G.J. Noordzij, ${ }^{a, b}$ Y.J.G. van den Boomen, ${ }^{b}$ C. Gilbert, ${ }^{b}$ D.J.P. van Elk, ${ }^{b}$ M. Roy, ${ }^{b}$ C.H.R.M. Wilsens, ${ }^{b}$ S. Rastogi ${ }^{b}$

RSC Polymer Chemistry, 2019, 10, 4049-4058

DOI: $10.1039 / c 9 p y 00463 g$

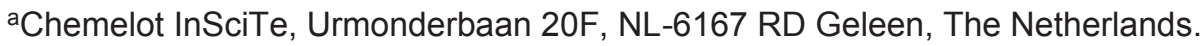

${ }^{b}$ Aachen-Maastricht Institute of Biobased Materials (AMIBM), Faculty of Science and Engineering, Maastricht University, Brightlands Chemelot Campus, 6167 RD Geleen, The Netherlands.

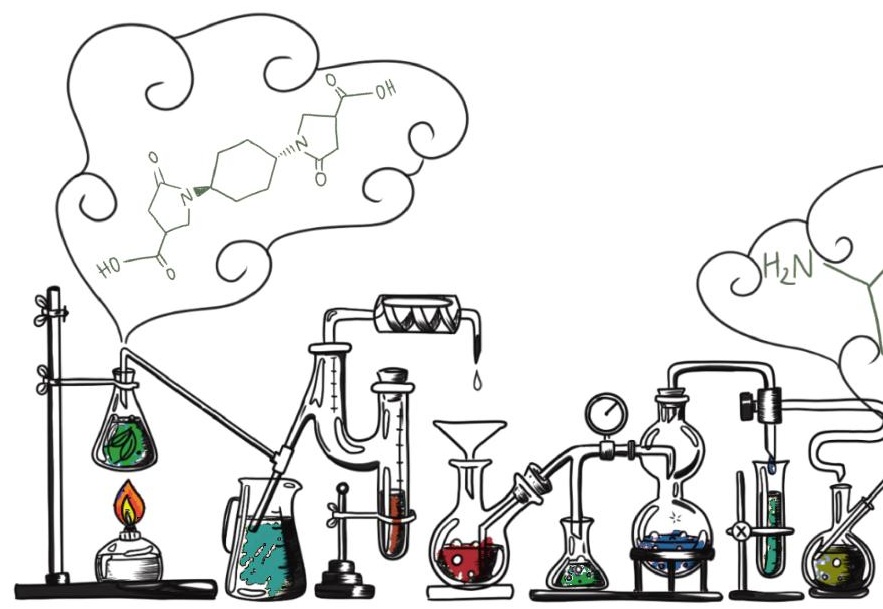




\section{Abstract}

This study reports, for the first time, semi-crystalline polymers based on bis-pyrrolidone dicarboxylic acids (BPDA) derived from renewable itaconic acid and various diamines. Rigid BPDA monomers could be obtained in high purity and yield from dimethyl itaconate, instead of the normally used itaconic acid as starting material. The reduction in melting point of dimethylBPDA monomers, compared to normal BPDA monomers, enables melt-polycondensation and thus avoiding the need of a solvent in the oligomerization step. Under standard polycondensation conditions, dimethyl-BPDA monomer with the rigid trans-cyclohexanediamine ( $t$-CH-BPDA $)$ could readily be polymerized with linear aliphatic diols $\left(\mathrm{C}_{6}\right.$ to $\left.\mathrm{C}_{12}\right)$ to yield high molecular weight polyesters. These polyesters are the first in their class to be reported semicrystalline, having a $T_{\mathrm{g}}$ between 65 to $33^{\circ} \mathrm{C}$, and a $T_{\mathrm{m}}$ between 213 to $184^{\circ} \mathrm{C}$, with increasing aliphatic chain length. Furthermore, even in the presence of hydrophilic pyrrolidone groups, semi-crystalline polyesters based on $t$-CH-BPDA showed high stability in water with no significant water uptake. Preliminary studies show that $t$-CH-BPDA monomers can also be polymerized into semi-crystalline polyamides, although insolubility and an unstable melt prevented accurate characterization of the synthesized polyamides. Though transcyclohexanediamine is currently not available from biomass, the potential biomass content in the described semi-crystalline polyesters range from $71 \%$ for $\mathrm{C}_{6}$ diol up to $81 \%$ for $\mathrm{C}_{12}$ diol.

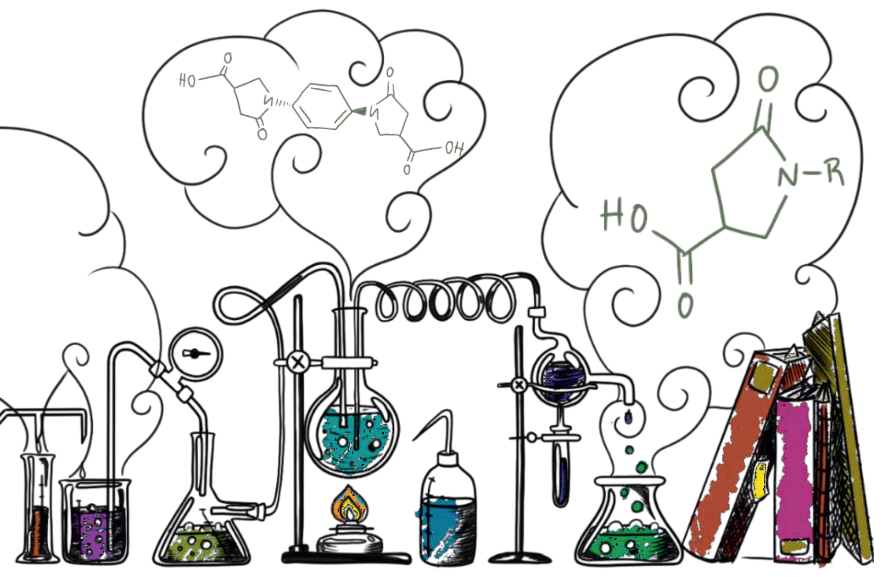




\section{Introduction}

The transition from non-renewable oil-based chemicals to renewable chemicals for the synthesis of polymers is an important topic having impact beyond the academic research due to growing concerns for the climate. ${ }^{1,2}$ At present, commercially available biobased polyesters with high melting temperatures include polylactides, polyhydroxyalkanoates, and poly(ethylene-2,5-furandicarboxylate). However, more polymers having high temperature stability are needed to facilitate the change from oil-based plastics to renewable biobased plastics. ${ }^{3-7}$ The U.S. Department of Energy reported 12 building blocks obtained from biomass which have high potential for high-value chemicals or materials. ${ }^{8,9}$ One of the listed cheap and readily available building blocks is itaconic acid, which is produced worldwide in a kiloton-scale by fermentation of glucose.

Itaconic acid (IA) is a di-acid with an $\alpha, \beta$-unsaturation in the backbone, and has proven useful for both radical ${ }^{10,11}$ and condensation polymerizations, ${ }^{12}$ or as component for preand post-modifications. ${ }^{12-19}$ However, one reaction mechanism involving the reactivity of itaconic acid remains relatively unexplored in the synthesis of polymers; When the $\alpha, \beta-$ unsaturation is reacted with an alkyl-amine species, an Aza-Michael addition takes place, which is immediately followed by ring-closure to form a $\mathrm{N}$-alkyl pyrrolidone-4-carboxylic acid ring (Scheme 5-1, left). This reaction, known since the 1850's, was first described in $1950,{ }^{20}$ and has been used in the production of surfactants. ${ }^{21-24}$ When a mono-functional amine (e.g. ethanolamine) is used, a mono-pyrrolidone ring is formed (Scheme 5-1, left, $\mathrm{R}=\mathrm{HOCH}_{2}$ ), and when a di-functional amine (e.g. ethylenediamine) is used, a bispyrrolidone-dicarboxylic acid (BPDA) is formed (Scheme 5-1, right). The resulting pyrrolidone based mono- or dicarboxylic acids can in turn be used in the polymerization of polyesters, ${ }^{25}$ poly(ester-amide)s, ${ }^{26,27}$ and polyamides. ${ }^{28-33}$

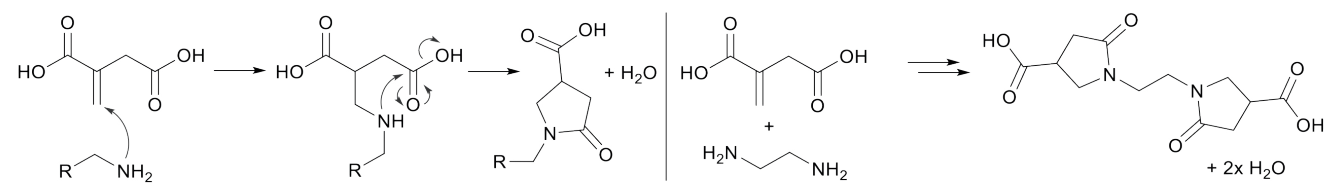

Scheme 5-1. Left: the Aza-Michael addition reaction between itaconic acid and an amine, followed by a ring-closing reaction to form an $\mathrm{N}$-alkyl-pyrrolidone-4-carboxylic acid. Right: the reaction of itaconic acid and a diamine species to form $N, N^{\prime}$-ethylene-bis-pyrrolidone-4-carboxylic acid.

A common trend with the incorporation of the pyrrolidone functionality in the polymer backbone, compared to aliphatic polymers, is an increase in $T_{\mathrm{g}}$, which can be attributed to the rigidity of the pyrrolidone ring. ${ }^{25,30,31}$ However, crystallization is significantly hampered, which can be attributed to the increased size of the pyrrolidone ring influencing looser packing of the polymer chains, ${ }^{26,32}$ and limited availability of favorable amide-amide interactions. Furthermore, the formation of the pyrrolidone ring is stereorandom, i.e. the pyrrolidone rings are obtained as a mixture of R/S, and hampers the stereoregularity and long-range order in the polymer chains. ${ }^{25}$ It is therefore not 
surprising that, to date, such pyrrolidone based homo-polymers with the ability to form chain-folded crystals have not been reported.

Previously reported polyamides, poly(ester-amide)s, and polyesters based on mono- or bis-pyrrolidones showed a high water uptake in polymer swelling/degradation tests, which significantly influenced thermal properties 27,29 and mechanical properties ${ }^{32}$ of the polymeric material. Furthermore, an increased water uptake led to hydrolytic degradation of BPDA-based polyesters in neutral water. ${ }^{25}$ However, for high temperature applications, both semi-crystallinity and a reduced affinity to water are desired properties. Therefore, in this work we report on novel BPDA monomers based on rigid cycloaliphatic diamines, including cis/trans 1,4-cyclohexanediamine, and cis/trans 1,3-cyclopentanediamine, of which the latter are potentially biorenewable. ${ }^{34,35}$ These monomers are polymerized with a series of diols and diamines with the goal to obtain materials with enhanced rigidity, regularity, and hydrophobicity. The synthesis, isolation, and characterization of various BPDA monomers from both itaconic acid as dimethylitaconate are described in detail. Furthermore, through careful selection of the diamine spacer in the BPDA monomer, semi-crystalline polyesters can be obtained with melting points ranging between $150{ }^{\circ} \mathrm{C}$ and $220^{\circ} \mathrm{C}$. These novel BPDA bearing polymers exhibit fast crystallization rates and a good hydrolytic stability in water, thereby generating new high-melting polymers with a high biobased content.

\section{Materials and Methods}

\section{Materials}

Itaconic acid, 1,4-butanediamine, 1,6-hexanediamine, 1,8-octanediamine, 1,10-decanediamine, 1,12-dodecanediamine, $p$-phenylenediamine, $m$-xylylenediamine, $p$-xylylenediamine, 1,4-butanediol, 1,6-hexanediol, 1,8-octanediol, 1,10-decanediol, 1,12-dodecanediol, 1,3-cyclopentanediol (cis/trans: 13:87), 1,4-cyclohexanediol (cis/trans: 33/67), Sn(II)2-ethylhexanoate, Ti(IV)isopropoxide, and Irganox 1010 were obtained from Sigma Aldrich. Dimethyl itaconate, cis-1,4-cyclohexanediamine, and trans-1,4-cyclohexanediamine were obtained from TCI. Cis-1,3-cyclopentanediamine and trans-1,3-cyclopentanediamine were obtained from iChemical. Standard laboratory solvents were obtained from Biosolve. DMF and toluene were distilled and stored over molsieves prior to use. Deuterated solvents were obtained from Buchem BV (Netherlands). The purchased compounds were used directly without further purification, unless otherwise specified.

\section{Characterization methods}

${ }^{1} \mathrm{H}-\mathrm{NMR}$ and ${ }^{13} \mathrm{C}-\mathrm{NMR}$ spectra were recorded with a Bruker Ultrashield 300 spectrometer $(300 \mathrm{MHz}$ magnetic field). NMR-samples were prepared by dissolving ca. $10 \mathrm{mg}$ of sample in $0.5 \mathrm{~mL}$ deuterated solvent, including dimethyl sulfoxide (DMSO- $\left.d_{6}\right)$, deuterated chloroform $\left(\mathrm{CDCl}_{3}\right)$, and deuterated trifluoroacetic acid $(d-T F A)$. All spectra were referenced against tetramethylsilane (TMS), or residual solvent peak from the deuterated solvent. LC-MS was measured on a LC-MS-2020 from Shimadzu equipped with a VisionHT C18 HL $1.5 \mu \mathrm{m}$ column. A gradient of analytical grade ACN and MiliQ water with $0.1 \%$ formic acid was used a mobile phase at $30{ }^{\circ} \mathrm{C}$, with a flowrate of $1 \mathrm{~mL} / \mathrm{min}$. Molecular weight $\left(M_{\mathrm{n}}\right.$, $M_{\mathrm{w}}$ ) and dispersity $(\Theta)$ of the polymers were calculated after gel permeation chromatography (GPC). HFIPGPC was measured on a PSS SECcurity GPC system using Agilent 1260 Infinity instrument technology. 
The GPC was equipped with a PFG Combination pre-column and two PFG Combination micro-columns. Distilled HFIP containing $0.019 \%$ sodium trifluoroacetate was used as mobile phase at $40{ }^{\circ} \mathrm{C}$, with a 0.3 $\mathrm{mL} / \mathrm{min}$ flow rate. $\mathrm{CHCl}_{3}-\mathrm{GPC}$ was measured on a Prominence-I LC-2030 equipped with a Shodex GPC $\mathrm{KF}-805 \mathrm{~L}$ column. Analytical grade $\mathrm{CHCl}_{3}$ was used as mobile phase at $40{ }^{\circ} \mathrm{C}$, with a flowrate of $1 \mathrm{~mL} / \mathrm{min}$. GPC samples were prepared by dissolving ca. $5 \mathrm{mg}$ of polymer in $1.5 \mathrm{~mL}$ of solvent overnight under constant shaking, the samples were filtered over a $0.2 \mu \mathrm{m}$ PTFE syringe filter prior to injection. Thermal stability of compounds were performed via thermogravimetric analysis (TGA) using a TA Instruments Q500. Experiments were performed under a nitrogen atmosphere with a heating rate of $10^{\circ} \mathrm{C} / \mathrm{min}$. Thermal transition temperatures of the polymers were analyzed via differential scanning calorimetry (DSC) using a TA Instruments DSC Q2000. Typically, two heating and cooling runs were performed at a rate of $10{ }^{\circ} \mathrm{C} / \mathrm{min}$, the first heating was used to erase any thermal history in the samples. The glass-transition temperature $\left(T_{\mathrm{g}}\right)$, peak melt-temperature $\left(T_{\mathrm{m}}\right)$, peak crystallization temperature $\left(T_{\mathrm{c}}\right)$, and cold-crystallization temperature $\left(T_{\mathrm{cc}}\right)$ were obtained from the second heating and cooling run. DSC samples were prepared by loading 3-5 mg oven-dried samples in Tzero Hermetic Aluminum pans. Polarized optical microscopy (POM) imaging was recorded on an Olympus BX53DP 26, equipped with a Linkam AFSX53 Hotstage. POM was used to determine melt-temperatures of samples which could not be measured via DSC (e.g. because of degradation at melt). POM samples were loaded on a microscopy slide and heated at a rate of $10^{\circ} \mathrm{C} / \mathrm{min}$, where the melt-temperature or range was determined by visual aid of the samples becoming anisotropic in nature.

\section{Synthesis methods}

\section{Synthesis of bis-pyrrolidone dicarboxylic acids (BPDA)}

The synthesis method to obtain aliphatic BPDA is described in previous work. ${ }^{27}$ In the current study, we expand the synthesis to more rigid diamine structures, as depicted in Scheme 5-2. The obtained BPDA monomers are abbreviated as $x x-B P D A$ where $x x$ denotes the type of diamine used: e.g. BPDA with 1,8 -octanediamine is abbreviated as $\mathrm{C}_{8}$-BPDA, BPDA with trans-1,4-cyclohexanediamine is abbreviated as $t$ - $\mathrm{CH}$-BPDA, and BPDA with $p$-phenylenediamine is abbreviated as $p$-Ph-BPDA. Details on synthesis, NMR characterization and thermal stability of the BPDA structures are supplied in the supporting information (SI) section for this chapter.
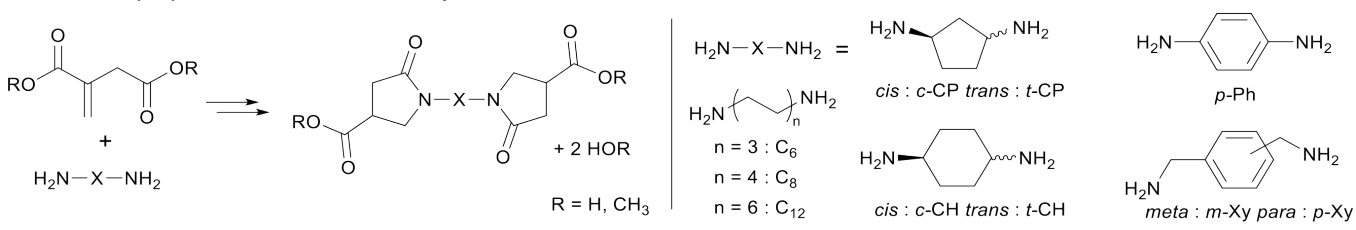

Scheme 5-2. Overview of diamines used in the synthesis of BPDA monomers with their corresponding abbreviations.

As a representative example, the synthesis of $\mathrm{N}, \mathrm{N}^{\prime}$-trans-1,4-cyclohexane-bis(pyrrolidone-4-carboxylic acid), abbreviated as $t$-CH-BPDA, is described: Itaconic acid $(9.11 \mathrm{~g}, 0.07 \mathrm{~mol})$ and trans-1,4-cyclohexanediamine $(1.14 \mathrm{~g}, 0.01 \mathrm{~mol})$ were loaded in a $100 \mathrm{~mL}$ round-bottom flask. The reaction mixture was stirred at $170{ }^{\circ} \mathrm{C}$ for $2-6$ hours in the melt, or until the reaction mixture was fully solidified. Recrystallization from water yielded the product as white solids $(2.1 \mathrm{~g}, 62 \%$ yield $)$.

\section{Synthesis of bis-pyrrolidone dicarboxylic acid - dimethyl-ester (BPDA-dm)}

A general synthesis pathway to obtain the dimethyl-esters of xx-BPDA (xx-BPDA-dm) from dimethylitaconate was developed. As a representative example, the synthesis of $t$-CH-BPDA-dm is described: dimethyl itaconate $(79.6 \mathrm{~g}, 0.5 \mathrm{~mol})$ and trans-1,4-cyclohexanediamine $(25 \mathrm{~g}, 0.22 \mathrm{~mol})$ were dissolved in $30 \mathrm{~mL}$ of methanol in a $250 \mathrm{~mL}$ round-bottom flask, equipped with a magnetic stirring bar. The reaction 
mixture was stirred at reflux overnight, and the product could directly be obtained as white crystals after cooling of the reaction mixture $(72.5 \mathrm{~g}, 90 \%$ yield $)$.

\section{Synthesis of $p-P h-B P D A-d m$}

Only $p$-Ph-BPDA-dm could not be obtained directly via the reaction of dimethyl-itaconate and $p$-phenylenediamine, hence methylation was performed on p-Ph-BPDA. p-Ph-BPDA (5.1 g, $15.3 \mathrm{mmol})$ was suspended in $100 \mathrm{~mL}$ of methanol with $2 \mathrm{~mL}$ of sulfuric acid in a $250 \mathrm{~mL}$ round-bottom flask. The suspension was refluxed for 72 hours, the methanol and formed water were removed in vacuo, the obtained product was re-suspended in $100 \mathrm{~mL}$ of methanol and refluxed for another 18 hours. The suspension was a grey slurry in deep purple liquid. After cooling, the product could be isolated as a grey solid by filtration $(4.45 \mathrm{~g}, 81 \%$ yield).

\section{Synthesis of organic salts with BPDA}

Organic salts of BPDA monomer with various di-amines were prepared by adding equal parts of a $0.1 \mathrm{M}$ solution of diamine in ethanol dropwise to a $0.1 \mathrm{M}$ solution of BPDA in ethanol at $70{ }^{\circ} \mathrm{C}$ (Scheme 5-3). Upon cooling white crystals of the organic salt crystallizes out, and could be isolated by filtration. Isolated yields ranged from $70 \%$ to $99.8 \%$, and ${ }^{1} \mathrm{H}$-NMR analysis showed near perfect stoichiometric amounts (details available in $\mathrm{SI}$ ).

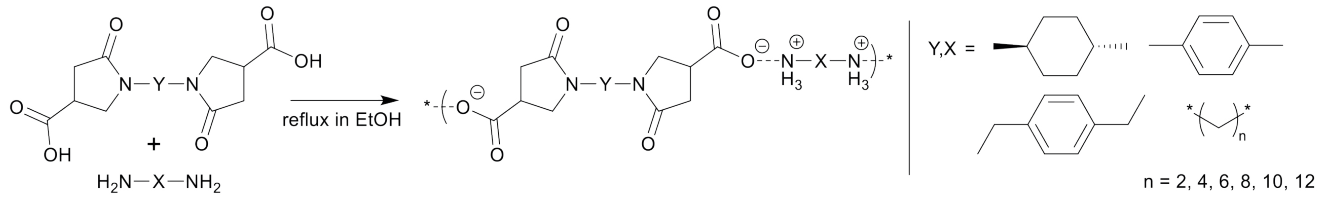

Scheme 5-3. Schematic overview of the synthesis of organic salts of xx-BPDA with various diamine structures.

\section{Melt-polycondensation of BPDA}

Melt-polycondensation of BPDA monomers with various diols was achieved via a two-step melt polymerization: an initial esterification (oligomerization) step, followed by transesterification (polymerization) in the melt under reduced pressure $(<0.01 \mathrm{mbar})$. Most BPDA-diol combinations and all BPDA-dm-diol mixtures formed a homogenous melt between 80 and $200{ }^{\circ} \mathrm{C}$, and could be polymerized without solvent. However some rigid BPDAs (e.g. $t$-CH-BPDA) could not melt below $200{ }^{\circ} \mathrm{C}$, and therefore DMF was used as solvent to achieve a homogenous solution to facilitate esterification. The polymers are abbreviated according to the BPDA and diol used: e.g. the polymer of $t$ - $\mathrm{CH}$-BPDA and 1,8-octanediol is abbreviated as poly $\left([t-\mathrm{CH}-\mathrm{BPDA}]-\mathrm{C}_{8}\right)$. Two representative examples are given below:

\section{Polymerization of $t$-CH-BPDA with 1,8-octanediol}

$t$-CH-BPDA (1.9 g, $5.4 \mathrm{mmol}$ ), 1,8-octanediol (0.9 g, $6.2 \mathrm{mmol}$ ), Irganox 1010 (40 mg, $0.011 \mathrm{mmol}$ ), were loaded in a $100 \mathrm{~mL} 3$-neck round-bottom flask, equipped with a $\mathrm{N}_{2} /$ vacuum inlet, vigreux column, and a heavy duty mechanical stirrer. The reaction mixture was purged 3 times with a vacuum $/ \mathrm{N}_{2}$ cycle before 8 $\mathrm{mL}$ of anhydrous DMF was added. The reaction mixture was slowly heated under light $\mathrm{N}_{2}$ flow to $120{ }^{\circ} \mathrm{C}$, after which the catalyst $\mathrm{Sn}$ (II)2-ethylhexanoate $(9.8 \mathrm{mg}, 0.025 \mathrm{mmol}$, in $0.1 \mathrm{~mL}$ anhydrous DMF) was added. For the oligomerization step, the reaction mixture was gradually heated to $160{ }^{\circ} \mathrm{C}$ where it was kept isothermal for 2 hours to allow for the generation and distillation of the condensate (water). Next, the temperature was gradually increased to $220^{\circ} \mathrm{C}$, where vacuum was applied for $6-10$ hours to allow buildup of molecular weight. When required, the progress of the reaction was monitored by sampling and determination of the molecular weight through GPC analysis, in order to identify the reaction time under vacuum. Generally, when the molecular weight $\left(M_{\mathrm{w}}\right)$ was lower than $10 \mathrm{~kg} / \mathrm{mol}$ after 6 hours under vacuum, 
the reaction was allowed to react for 4 hours more. The final polymer was obtained as an off-white or light orange solid and was used without further purification.

\section{Polymerization of $t-\mathrm{CH}-\mathrm{BPDA}-\mathrm{dm}$ with 1,8-octanediol}

$t$-CH-BPDA-dm (7.2 g, $19.6 \mathrm{mmol}), 1,8$-octanediol (3.46 g, $23.5 \mathrm{mmol})$, Irganox 1010 (40 mg, $0.011 \mathrm{mmol})$, were loaded in a $100 \mathrm{~mL} 3$-neck round-bottom flask, equipped with a $\mathrm{N}_{2} /$ vacuum inlet, vigreux column, and a heavy duty mechanical stirrer. The polymer was synthesized using the same protocol as reported in the polymerization of $t$-CH-BPDA with 1,8-octanediol section, with the exception that no solvent was used and that $\mathrm{Ti}(\mathrm{IV})$ isopropoxide (31 $\mathrm{mg}, 0.11 \mathrm{mmol}$, in $0.1 \mathrm{~mL}$ anhydrous toluene or DMF) was used as catalyst. The polymer was obtained as an off-white or light orange solid and was used without further purification.

\section{Solid-state polymerization of $t-C H-B P D A$ - diamine salts}

Conditions for solid-state polymerization of the $t$-CH-BPDA-diamine salts (Scheme 5-6) were obtained by determining the melting points $\left(T_{\mathrm{m}}\right)$ of the salts via POM, and the onset of weight-loss ( $\left.T_{\mathrm{ons}}\right)$ via TGA (5 wt \% loss). Next, the polymerization temperature was set a little below $T_{\text {ons }}$, and well below $T_{\mathrm{m}}$ of the salt to ensure solid-state conditions. The polymerization was performed in a HPLC vial with $\pm 20 \mathrm{mg}$ of the salt. The HPLC vial was placed in a stainless steel reactor, purged 3 times with vacuum $/ \mathrm{N}_{2}$ cycle, and gradually heated for 4-8 hours under vacuum at the desired polymerization temperature. The observed weight-loss during solid-state polymerization should be equal to the wt $\%$ of $2 n-1 \mathrm{H}_{2} \mathrm{O}$, and can serve as a measure of degree of polymerization. 


\section{Results and Discussion}

\section{$B P D A$ and BPDA-dm monomer synthesis}

An overview of the used monomers is provided in Scheme 5-2 and their synthesis methods are listed in the experimental section. The monomer synthesis was based on earlier work, where BPDA monomers were synthesized from IA with aliphatic diamines (ratio of 2.1:1) in bulk at $130{ }^{\circ} \mathrm{C}$ (Table 5-1). ${ }^{27}$ However, when the same protocol was applied with rigid diamines such as trans-1,4-cyclohexane $(t-\mathrm{CH})$ and paraphenylenediamine $(p-\mathrm{Ph})$, the reaction mixture either did not melt, or solidified quickly during reaction, leading to poor yields. Based on previous work by Zilkha et al. ${ }^{28}$ the ratio IA : diamine was raised from $2.1: 1$ to $5: 1$, and the reaction temperature was increased to $170{ }^{\circ} \mathrm{C}$ to melt IA $\left(T_{\mathrm{m}} 162-164{ }^{\circ} \mathrm{C}\right)$ and thus to ensure a homogeneous mixture. The reaction was complete between 2-6 hours and the product could be isolated after recrystallization from boiling water. The yield (based on conversion of diamine) of $p$-PhBPDA was nearly quantitative. In contrast, the yield of $t-\mathrm{CH}-\mathrm{BPDA}$ remained low at $62 \%$, with significant amount of side-product observed in LC-MS and NMR. The side product was isolated via recrystallization in ethyl acetate/methanol $(40 / 60 \mathrm{v} / \mathrm{v} \%)$, and characterized with 2D-NMR (Figure 5-1).

Table 5-1. Overview of BPDA monomer synthesis yield, melting point, and thermal stability. *) Melt in first heating only ${ }^{* *}$ ) Yield based on the conversion of $p$-Ph-BPDA to $p$-Ph-BPDA-dm.

\begin{tabular}{|c|c|c|c|}
\hline monomer & Yield & $T_{\mathrm{m}}\left({ }^{\circ} \mathrm{C}\right)$ & $T_{\mathrm{d}}\left({ }^{\circ} \mathrm{C}\right)$ \\
\hline$C_{6}-B P D A$ & $75 \%$ & 181 & 266 \\
\hline$C_{8}-B P D A$ & $85 \%$ & 142 & 267 \\
\hline$C_{12-B P D A}$ & $90 \%$ & 133 & 259 \\
\hline$t-C H-B P D A$ & $62 \%$ & 307 & 327 \\
\hline$t-C H-B P D A-d m$ & $90 \%$ & 197 & 284 \\
\hline$c-C H-B P D A$ & n.d. & $>T_{\mathrm{d}}$ & 287 \\
\hline$c-C H-B P D A-d m$ & $63 \%$ & $117^{*}$ & 269 \\
\hline$t-C P-B P D A$ & $88 \%$ & $>T_{\mathrm{d}}$ & 187 \\
\hline$t-C P-B P D A-d m$ & $53 \%$ & $91^{*}$ & 273 \\
\hline$C-C P-B P D A$ & $93 \%$ & $>T_{\mathrm{d}}$ & 181 \\
\hline$c-C P-B P D A-d m$ & $55 \%$ & $79^{*}$ & 270 \\
\hline$p-P h-B P D A$ & $99 \%$ & $>T_{\mathrm{d}}$ & 295 \\
\hline$p-P h-B P D A-d m$ & $81 \%$ ** & 202 & 283 \\
\hline$m-X y-B P D A-d m$ & $77 \%$ & n.o. & 302 \\
\hline$p-X y-B P D A-d m$ & $74 \%$ & 127 & 267 \\
\hline
\end{tabular}

The side reaction was identified to be a result of the amidation reaction between itaconic acid and $t-\mathrm{CH}$, followed by a ring-closing step to form an imide (pathway $\boldsymbol{b}$, Scheme 5-4), rather than the desired pathway a to form the pyrrolidone ring. In addition, isomerization 
of the double bond to the internal position (66\%) is observed, as is evident in the strong methyl-signal 7 in NMR (Figure 5-1).

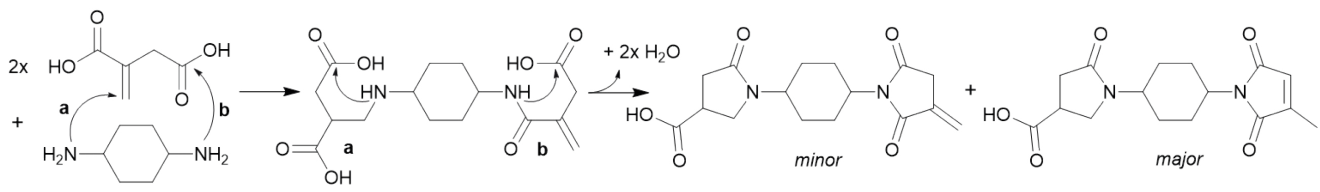

Scheme 5-4. Schematic difference of desired Aza-Michael addition reaction (a) and undesired amidation reaction (b). Pathway a forms the desired pyrrolidone ring, whereas pathway $\mathbf{b}$ forms an undesired imide, which has two isomers.

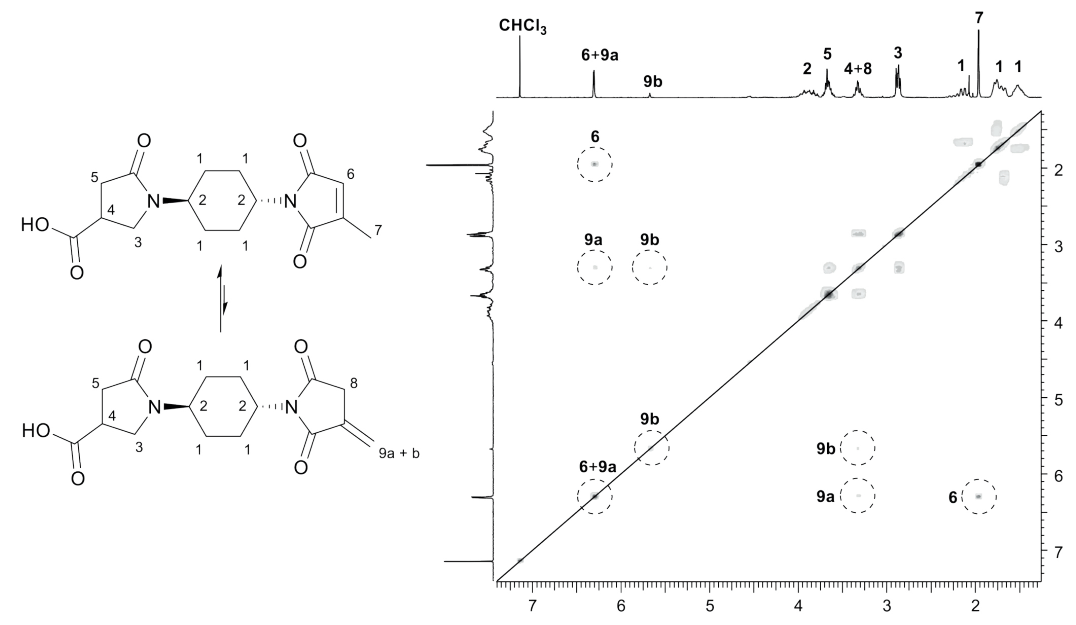

Figure 5-1. COSY $2 \mathrm{D}\left({ }^{1} \mathrm{H}-{ }^{1} \mathrm{H}\right)-\mathrm{NMR}$ analysis of the amidation side-product in the synthesis of $t$-CH-BPDA at high temperatures. The amidation side product isomerizes to form mainly the top left structure $(66 \%)$, as is evident in the ratio of protons of $\mathrm{CH} 6 \& 9$ (right, circled).

Several BPDA monomers either exhibit an unstable melt (e.g. c-CP-BPDA), or a too high melting point (e.g. $t$-CH-BPDA) to be used in typical oligomerization and meltpolycondensation conditions between $180-200^{\circ} \mathrm{C}$. In order to achieve a homogenous mixture, and therefore maximum reactivity, during the oligomerization step some DMF was added. Even though addition of DMF ensured successful polycondensation for several high-melting BPDA monomers (Table 5-3 and Table 5-4), the use of DMF is highly undesired due to toxicological and environmental concerns. Additionally, trace amounts of DMF were detectable in NMR for several polymers where DMF was used either as solvent for the BPDA monomer or for addition of the catalyst (SI). To avoid the use of DMF, a suppression in melting temperature of the BPDA monomers was required. Therefore, dimethyl esters of the BPDA monomers were synthesized using dimethyl itaconate $(\mathrm{dm}-\mathrm{I})$ (Scheme 5-2, $\mathrm{R}=\mathrm{CH}_{3}$ ) as starting material instead of IA. The synthesis method of BPDA-dm monomers is based on a recent study by Clark et al. ${ }^{13}$ who showed that $\mathrm{dm}-\mathrm{I}$ readily isomerizes to a less reactive mesaconate species for up to $15 \%$. To 
account for this reaction, a slight excess of dm-I was used: $\mathrm{dm}-\mathrm{I}$ and the corresponding diamine were mixed in a 2.5:1 ratio and refluxed in methanol overnight. Typically, the BPDA-dm monomers with rigid diamines ( $t-\mathrm{CH}-\mathrm{BPDA}-\mathrm{dm}, p-\mathrm{Xy}-\mathrm{BPDA}-\mathrm{dm}$ ) crystallized out of solution upon cooling, and were isolated in high yields. The BPDA-dm monomers with less rigid diamines (cis-1,4-cyclohexanediamine (c-CH-BPDA-dm), cis-1,3-cyclopentanediamine (c-CP-BPDA-dm), and trans-1,3-cyclopentanediamine $(t$-CP-BPDA-dm)) had to be purified via column chromatography. The only exception for this synthesis method is for $p$-Ph-BPDA-dm: The synthesis of dm-I with p-phenylenediamine stopped after $50 \%$ conversion, and $p$-Ph-BPDA-dm could not be isolated. Instead, $p$-Ph-BPDA was methylated by refluxing in methanol with catalytic sulfuric acid which yielded $p$-Ph-BPDA-dm in high conversion. The results of the monomer synthesis and is summarized in Table 5-1, whereas their analysis is provided in detail in the SI.

The advantage of the direct synthesis of BPDA-dm monomers from rigid diamines is clear: for example the $t$-CH-BPDA-dm monomer is readily obtained via crystallization during cooling, requires little solvent, and avoids the use of toxic solvents (e.g. DMF) needed for polymerization due to the significantly reduced melting temperature of the monomer. On the other hand, for less rigid diamines, isolation via column chromatography becomes necessary, potentially negating the positive effects listed above. To determine whether the BPDA or BPDA-dm monomer is preferred, the following rule of thumb can be applied: The BPDA dicarboxylic acid can be used directly in meltpolycondensation reaction when it has a stable melt below $200{ }^{\circ} \mathrm{C}$. However, when the BPDA monomer exhibits an unstable melt, or melts at temperatures higher than $200{ }^{\circ} \mathrm{C}$, synthesis of the dimethyl ester is preferred as this lowers the monomer melting temperature and thereby allows for stable a melt-polymerization in the absence of DMF. For example, $t$-CH-BPDA melts at $307^{\circ} \mathrm{C}$, whereas $t-\mathrm{CH}-\mathrm{BPDA}-\mathrm{dm}$ melts at $197^{\circ} \mathrm{C}$ and is thus preferred for usage in melt-polycondensation. The synthesis of the polymers reported in this study were performed following to the above-mentioned guidelines.

Semi-crystalline BPDA-based polyesters: effect of change of diamine

An excellent previous study by Miller et al. ${ }^{25}$ showed that polyesters based on aliphatic BPDA monomers (e.g. C2-BPDA) with 1,6-hexanediol were amorphous. To understand the role of the structure of the diamine on the properties of these polyesters, the BPDA monomer selection was extended with those based on cycloaliphatic diamines such as $c-\mathrm{CP}, t-\mathrm{CP}, c-\mathrm{CH}, t-\mathrm{CH}$ and aromatic diamines such as $p-\mathrm{Ph}, p$-xylylene diamine $(p-\mathrm{Xy})$ and $m$-xylylene diamine $(m-X y)$, (Scheme $5-2)$. Both the aliphatic, cycloaliphatic, and aromatic BPDA monomers were polymerized with linear aliphatic diols such as 1,6-hexanediol, and the resulting polymers were subjected to GPC and DSC analysis to identify their molecular weight and thermal behavior (Table 5-2). Complementary to the work of Miller et al. ${ }^{25}$ the polyesters containing aliphatic BPDA with linear diols are found to be amorphous, with a clear trend in decreasing glass transition temperature $\left(T_{g}\right)$ with 
the increase in spacer length of the diol. The incorporation of aromatic diamines in the BPDA structure was expected to increase rigidity of the BPDA segments, thereby imposing a higher stereoregularity among the polymeric backbone. Furthermore, the aromatic moieties are expected to undergo favorable $\pi-\pi$ stacking. Indeed, such effect of the rigidity of the $p$-phenylene group is detected by the strong increase in $T_{\mathrm{g}}$ to $83^{\circ} \mathrm{C}$. The addition of methylene spacers on the phenyl ring yields a slightly lowered $T_{\mathrm{g}}$ of approximately $50{ }^{\circ} \mathrm{C}$ for polymers based on $m$-Xy and $p$-Xy.

Table 5-2. Overview of polymer properties of BPDA with a changing diamine structure.

\begin{tabular}{|c|c|c|c|c|c|c|}
\hline$B P D A(-d m)$ & diol & $\begin{array}{c}M_{\mathrm{n}} \\
(\mathrm{g} / \mathrm{mol})\end{array}$ & $\begin{array}{c}M_{\mathrm{w}} \\
(\mathrm{g} / \mathrm{mol})\end{array}$ & $\bigoplus$ & $T_{\mathrm{g}}\left({ }^{\circ} \mathrm{C}\right)$ & $T_{\mathrm{m}}\left({ }^{\circ} \mathrm{C}\right)$ \\
\hline$C_{2}$ & $\mathrm{C}_{6}$ & 7900 & 18700 & 2.38 & 0 & amorphous \\
\hline$C_{12}$ & $\mathrm{C}_{6}$ & 10700 & 23500 & 2.19 & -22 & amorphous \\
\hline$c-C P-d m$ & $\mathrm{C}_{6}$ & 9200 & 22900 & 2.43 & 34 & amorphous \\
\hline$t-C P-d m$ & $\mathrm{C}_{6}$ & 26900 & 73300 & 2.72 & 32 & amorphous \\
\hline$p-P h$ & $\mathrm{C}_{6}$ & 9800 & 25100 & 2.56 & 84 & amorphous \\
\hline$m-X y$ & $\mathrm{C}_{6}$ & 9300 & 21700 & 2.34 & 26 & amorphous \\
\hline$p-X y-d m$ & $\mathrm{C}_{8}$ & 24700 & 88700 & 3.59 & 21 & amorphous \\
\hline$c-C H-d m$ & $\mathrm{C}_{4}$ & 8000 & 13100 & 1.63 & 50 & amorphous \\
\hline$c-C H-d m$ & $\mathrm{C}_{6}$ & 2900 & 3500 & 1.22 & 28 & amorphous \\
\hline$t-C H-d m$ & $\mathrm{C}_{6}$ & 26000 & 50300 & 1.93 & 65 & 213 \\
\hline
\end{tabular}

With the incorporation of $t-\mathrm{CP}$ or $c-\mathrm{CP}$ moieties in the BPDA structure, amorphous polyesters are obtained with a higher $T_{\mathrm{g}}$ than linear diamines, though there is no clear cis/trans effect observed in the $T_{\mathrm{g}}$ (Figure 5-2, black and red line). The absence of a cis/trans effect in $T_{\mathrm{g}}$ is likely due to the preferred envelope-conformation of both cyclopentane isomers, ${ }^{36}$ which therefore exhibit similar rigidity. Previous work on polyesters based on 1,3-cyclopentanediol ${ }^{37}$ showed a typical cis/trans effect, though only in the melting point $\left(T_{\mathrm{m}}\right)$. In contrast, polymers containing cyclohexane moieties do show a strong cis/trans effect in $T_{\mathrm{g}}$ : polymers containing $t-\mathrm{CH}$ have a much higher $T_{\mathrm{g}}$ than polymers containing $\mathrm{c}-\mathrm{CH}$ (Figure 5-2, blue and green line, respectively). This is explained by the preferred chair conformation for trans-isomers, which has a higher rigidity than the twisted boat conformation for the cis-isomers. These findings are coherent with other studies for the cis/trans effect in polymers bearing cyclohexane rings such as 1,4-cyclohexanediamine, ${ }^{38}$ 1,4-cyclohexanedicarboxylic acid, ${ }^{39-41}$ and 1,4cyclohexanedimethanol. ${ }^{42}$ Furthermore, as will be elaborated later in this work; the incorporation of $c-\mathrm{CH}$ moieties yield solely amorphous polyesters, whereas the presence of $t-\mathrm{CH}$ moieties enables polymer crystallization. This leads to the first reported semi-crystalline polymer based on BPDA monomers, as is evident by the melting temperature of $213^{\circ} \mathrm{C}$ for poly([t-CH-BPDA]-C6) (Table 5-2). 


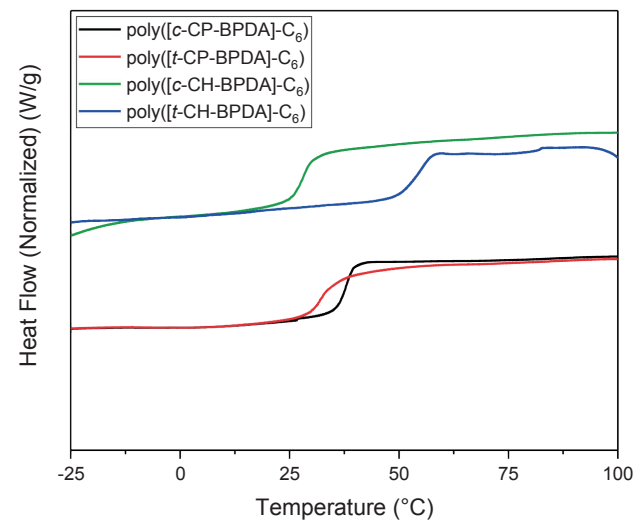

Figure 5-2. DSC thermograms (second heating run) displaying the cis/trans effect in $T_{\mathrm{g}}$ for polymers containing 1,6-hexanediol ( $\left.\mathrm{C}_{6}\right)$ and $c$-CP-BPDA-dm (black), $t$-CP-BPDA-dm (red), $c-C H$-BPDA-dm (green), and $t$-CH-BPDA-dm (blue).

Semi-crystalline BPDA-based polyesters: effect of change in diol

Semi-crystallinity is observed solely for polymers containing $p$-Ph-BPDA or $t$-CH-BPDA. Therefore, for these polymers, the effect of varying the diol on the polymer properties has been studied (Scheme 5-5). Polyesters synthesized from $p$-Ph-BPDA and $p$-Ph-BPDA$\mathrm{dm}$ with varying diol structures have been prepared, where the results are summarized in Table 5-3. Similarly, an overview of the polyesters synthesized from $t$-CH-BPDA and $t$-CH-BPDA-dm with varying diols is provided in Table 5-4.
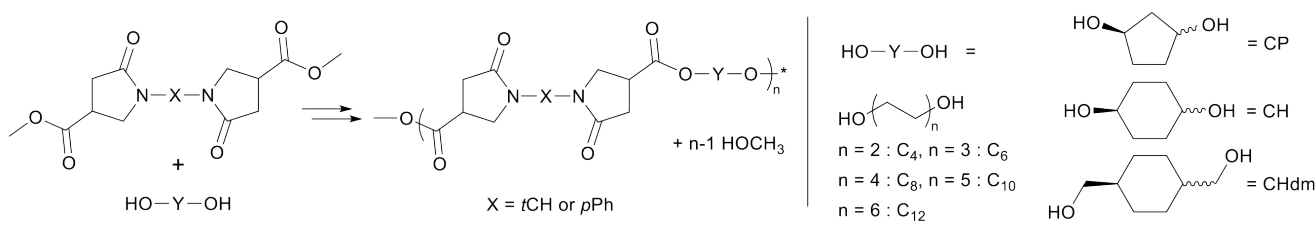

Scheme 5-5. Overview of polymerization of $t$-CH-BPDA-dm and $p-P h-B P D A-d m$ with various diol structures.

Table 5-3. Overview of properties of polymers based on $p$-Ph-BPDA and $p$-Ph-BPDA-dm with varying diols.

\begin{tabular}{|c|c|c|c|c|c|c|}
\hline$B P D A-d m$ & diol & $\begin{array}{c}M_{\mathrm{n}} \\
(\mathrm{g} / \mathrm{mol})\end{array}$ & $\begin{array}{c}M_{\mathrm{w}} \\
(\mathrm{g} / \mathrm{mol})\end{array}$ & $\boldsymbol{\boxplus}$ & $T_{\mathrm{g}}\left({ }^{\circ} \mathrm{C}\right)$ & $T_{\mathrm{m}}\left({ }^{\circ} \mathrm{C}\right)$ \\
\hline$p-P h$ & $\mathrm{C}_{6}$ & 10800 & 25400 & 2.36 & 84 & amorphous \\
\hline$p-P h$ & $\mathrm{C}_{8}$ & 7300 & 15400 & 2.1 & 58 & amorphous \\
\hline$p-P h-d m$ & $\mathrm{C}_{10}$ & 40400 & 87700 & 2.2 & 41 & 153 \\
\hline$p-P h-d m$ & $\mathrm{C}_{12}$ & 13300 & 33100 & 2.48 & 19 & 149 \\
\hline$p-P h$ & $\mathrm{CP}$ & - & - & - & 120 & $T_{\mathrm{d}}>T_{\mathrm{m}}>220$ \\
\hline$p-P h$ & $\mathrm{CHdm}$ & - & - & - & 131 & $T_{\mathrm{d}}>T_{\mathrm{m}}>260$ \\
\hline
\end{tabular}

As is evident from Table 5-3, polyesters based on p-Ph-BPDA(-dm) and long aliphatic diols (e.g. $\left.\mathrm{C}_{10}, \mathrm{C}_{12}\right)$ are semi-crystalline with a melting temperature around $150{ }^{\circ} \mathrm{C}$. 
However, only poly([p-Ph-BPDA]-C $\left.{ }_{12}\right)$ exhibits extensive cold-crystallization $\left(72{ }^{\circ} \mathrm{C}\right)$ when heated from the glassy state, followed by subsequent melting at $149^{\circ} \mathrm{C}$ (Figure 5-3). Polyesters based on $p-\mathrm{Ph}-\mathrm{BPDA}(-\mathrm{dm})$ and rigid diols $(\mathrm{CP}, \mathrm{CH})$ readily crystallized during polymerization and were off-white hard solids when isolated from the reactor. Unfortunately, degradation occurs immediately upon melting of these polymers, which prevents accurate identification of their melting temperature. Additionally, these polyesters are insoluble in the normally used GPC solvents, including DMF at elevated temperatures, preventing subsequent molecular weight analysis. Nevertheless, this data clearly suggest that, with the right selection of diols, polymers based on $p$-Ph-BPDA($\mathrm{dm}$ ) can be semi-crystalline in nature.

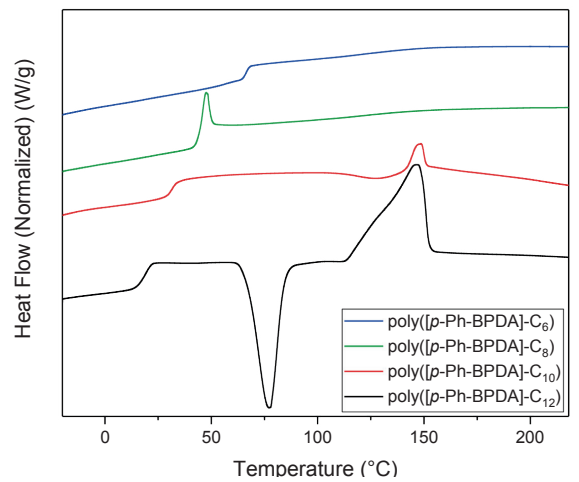

Figure 5-3. DSC thermograms (second heating run) of polyesters from $p$-Ph-BPDA(-dm) with linear aliphatic diols $\mathrm{C}_{6}$ (blue), $\mathrm{C}_{8}$ (green), $\mathrm{C}_{10}$ (red), and $\mathrm{C}_{12}$ (black).

In contrast to $p$-Ph-BPDA $(-\mathrm{dm})$ based polymers, all polyesters based on $t$-CH-BPDA $(-\mathrm{dm})$ and aliphatic diols were semi-crystalline. Unfortunately, the polymer based on $t$-CH-BPDA-dm and $\mathrm{C}_{4}$-diol could not be obtained in high molecular weight as it rapidly crystallizes during polymerization. Polymers from $t$-CH-BPDA-dm and longer linear aliphatic diols $\left(\geq \mathrm{C}_{6}\right)$ could readily be polymerized into high molecular weight polyesters under the used reaction conditions. From Table 5-4, it is evident that the polymers display a decreasing trend in both $T_{\mathrm{g}}\left(65\right.$ to $\left.33^{\circ} \mathrm{C}\right)$ and $T_{\mathrm{m}}\left(213\right.$ to $\left.184^{\circ} \mathrm{C}\right)$ with increasing spacer length of the diol. Similar to $p$-Ph-BPDA, the polymerization of $t$-CH-BPDA with cycloaliphatic diols led to low molecular weight species that degraded upon melting at much higher temperatures than the polymerization temperature, indicating that the used polymerization conditions are not suitable for these type of polymers due to the unstable melt. 
Table 5-4. Overview of properties of polymers based on $t$ - $\mathrm{CH}-\mathrm{BPDA}$ and $t$-CH-BPDA-dm with varying diols.

\begin{tabular}{|c|c|c|c|c|c|c|}
\hline$B P D A-d m$ & diol & $\begin{array}{c}M_{\mathrm{n}} \\
(\mathrm{g} / \mathrm{mol})\end{array}$ & $\begin{array}{c}M_{\mathrm{w}} \\
(\mathrm{g} / \mathrm{mol})\end{array}$ & $\bigoplus$ & $T_{\mathrm{g}}\left({ }^{\circ} \mathrm{C}\right)$ & $T_{\mathrm{m}}\left({ }^{\circ} \mathrm{C}\right)$ \\
\hline$t-C H-d m$ & $\mathrm{C}_{4}$ & 3300 & 5600 & 1.66 & n.o. & $T_{\mathrm{d}}>T_{\mathrm{m}}$ \\
\hline$t-C H-d m$ & $\mathrm{C}_{6}$ & 26000 & 50300 & 1.93 & 65 & 213 \\
\hline$t-C H-d m$ & $\mathrm{C}_{8}$ & 46300 & 110600 & 2.39 & 51 & 199 \\
\hline$t-C H-d m$ & $\mathrm{C}_{10}$ & 23400 & 56900 & 2.43 & 39 & 189 \\
\hline$t-C H-d m$ & $\mathrm{C}_{12}$ & 13300 & 33100 & 2.95 & 33 & 184 \\
\hline$t-C H$ & $\mathrm{CH}$ & 1900 & 2400 & 1.26 & 82 & 239 \\
\hline$t-\mathrm{CH}$ & $\mathrm{CP}$ & 3200 & 4000 & 1.25 & n.o. & $T_{\mathrm{d}}>T_{\mathrm{m}}$ \\
\hline
\end{tabular}

The characteristic cold-crystallization and melting behavior of polymers based on $t$ - $\mathrm{CH}$ BPDA-dm and linear aliphatic diols is displayed in Figure 5-4. Heating of the polymers, after quenching into the glassy state from the melt, yield DSC thermograms that display a characteristic glass transition followed by cold crystallization upon further heating. Furthermore, all $t$-CH-BPDA-dm based polymers display a melting endotherm, followed by an exothermic transition, before displaying their final melting temperature with an endothermic transition approximately $25{ }^{\circ} \mathrm{C}$ higher than the first endotherm. This behavior could originate from a crystal-to-crystal transition ${ }^{43}$ or from the melting and recrystallization process of small and defected spherulites. Though the melting behavior of the polymers based on $t$-CH-BPDA-dm is highly interesting, in-depth DSC and XRD crystallization studies are out of the scope of this paper and will be discussed in future communications. Lastly, all $t$-CH-BPDA-dm based polymers display a distinct crystallization exotherm during cooling at a rate of $10^{\circ} \mathrm{C} / \mathrm{min}$, as is shown in the SI.

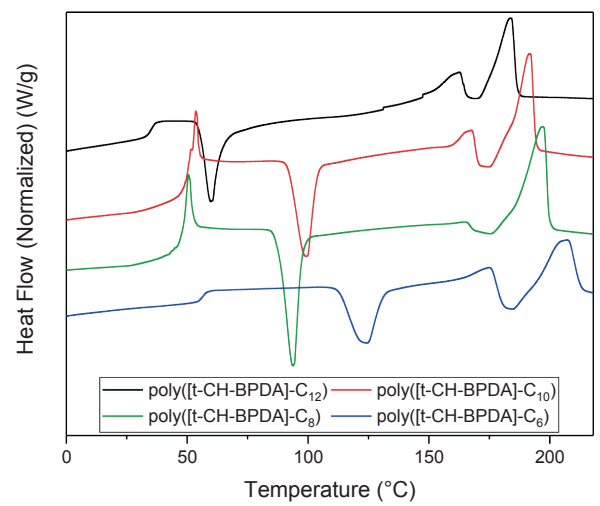

Figure 5-4. DSC thermograms (first heating run) of quenched polyesters from $t$-CH-BPDA-dm with linear aliphatic diols $\mathrm{C}_{6}$ (blue), $\mathrm{C}_{8}$ (green), $\mathrm{C}_{10}$ (red), and $\mathrm{C}_{12}$ (black). All traces contain the characteristic transitions corresponding to the glass transition, cold crystallization and the presence of two melt-endotherms. 


\section{Hydrolytic stability}

Previously reported polyesters based on mono- and bis-pyrrolidones exhibited a high hydrolysis rate in water, with a $28 \%$ loss in molecular weight after 30 days, and full hydrolysis within one year. ${ }^{25}$ To study the effect of semi-crystallinity on water absorption and hydrolysis, a square piece $\left(5^{*} 5^{*} 1 \mathrm{~mm}\right)$ of poly $\left([t-\mathrm{CH}-\mathrm{BPDA}]-\mathrm{C}_{10}\right)$ was shaken in water with a $\mathrm{pH}$ of $4.0(\mathrm{HCl})$ for 4 weeks. After 4 weeks, no shape change (e.g. swelling) was observed. TGA analysis shows minimal absorption of water compared to the dry sample $\left(1.2 \%\right.$ vs $0.7 \%$ weight loss up to $\left.200{ }^{\circ} \mathrm{C}, \mathrm{SI}\right)$, and $\mathrm{GPC}$ traces show a decrease in molecular weight $\left(M_{\mathrm{w}}\right)$ of roughly $10 \%$ (Figure 5-5). These preliminary results indicate that semi-crystallinity and the enhanced polymer rigidity can improve the hydrolytic stability of BPDA-based polyesters, at least compared to the previously reported amorphous BPDA-based polyesters.

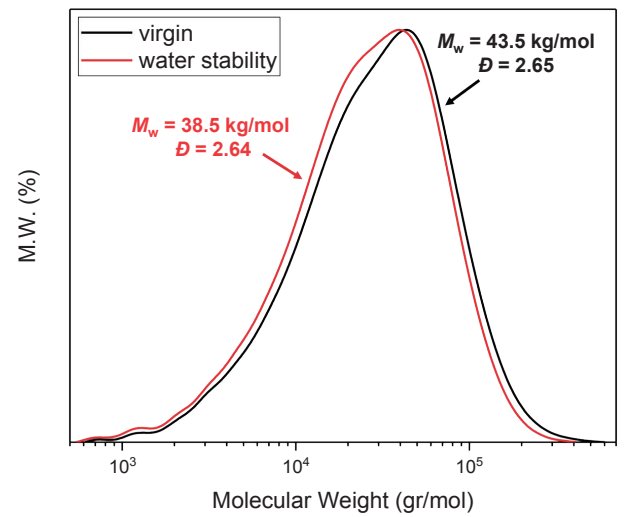

Figure 5-5. GPC traces of poly $\left([t-\mathrm{CH}-\mathrm{BPDA}]-\mathrm{C}_{10}\right)$ of the virgin material (black), and after 4 weeks shaking in water with a pH 4.0 (red).

\section{BPDA-based polyamides}

Previous polyamide synthesis via organic salts directly from itaconic acid and various diamines led to amorphous mono-pyrrolidone based polyamides. ${ }^{30-33}$ Similarly, the synthesis of BPDA-based polyamides is reported for the polymerization of aliphatic BPDA $^{29}$ and aromatic BPDA ${ }^{28}$ with various diamines, though all reported polyamides were amorphous. As polyesters based on $t$ - $\mathrm{CH}$-BPDA are semi-crystalline, polyamides based on $t$ - $\mathrm{CH}$-BPDA were synthesized to identify whether these are also capable of forming chain-folded crystals. Organic salts have been made of $t$-CH-BPDA with various diamine structures, and were polymerized under solid-state conditions (Scheme 5-6). To ensure solid-state polymerization, the melting points $\left(T_{\mathrm{m}}\right)$ of the salts were determined via POM, and the onset of weight-loss ( $\left.T_{\text {on }}\right)$ was determined via TGA at 5 wt $\%$ loss. The polymerization temperature was set a little below $T_{\text {on, }}$ and well below $T \mathrm{~m}$ of the salt. Analysis of the salt melting behavior, and the data used for the identification of the optimum polymerization temperature is described in the SI. 


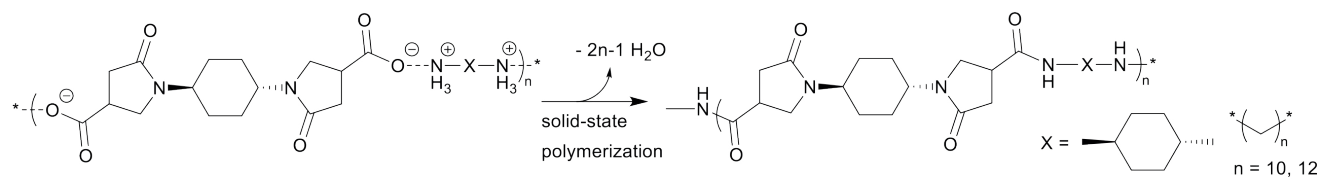

Scheme 5-6. Schematic overview of solid-state polymerization of BPDA-based polyamides from the organic salts of $t$-CH-BPDA with a diamine.

The solid-state polymerization results with $t$-CH-BPDA and various diamines are summarized in Table 5-5. In contrast to the previously reported BPDA-based polyamides, these polyamides were completely insoluble in any GPC solvent, including DMF at 140 ${ }^{\circ} \mathrm{C}$. Though characterization of the obtained polyamides was not successful, their insolubility and increased temperature resistance (SI) suggest that polyamides have successfully been formed. The polymerization of $t-\mathrm{CH}-\mathrm{BPDA}$ with $\mathrm{C}_{10}$ and $\mathrm{C}_{12}$ diamine rendered transparent brown solids, with the transparency suggesting the absence of crystallinity. This implies that, though polyesters based on $t-\mathrm{CH}-\mathrm{BPDA}(-\mathrm{dm})$ and aliphatic diols are semi-crystalline, semi-crystalline polyamides are not obtained when exchanging the aliphatic diols with the corresponding aliphatic diamines. Possibly, a competition between inter- and intra-molecular hydrogen bonding between the amide-groups and pyrrolidone moieties suppresses the crystallization process. In contrast, the polymerization of $t-\mathrm{CH}$-BPDA with $t-\mathrm{CH}$ diamine rendered light brown solids, suggesting that the obtained polyamide is semi-crystalline. Indeed, during heating, the obtained polyamide showed melting close to $300{ }^{\circ} \mathrm{C}$ followed by immediate degradation.

Table 5-5. Overview of solid-state polymerization from the organic salts of BPDA with various diamines.") Ratio determined by NMR, available in SI.

\begin{tabular}{|c|c|c|c|c|c|c|c|c|}
\hline$B P D A$ & diamine & $\begin{array}{c}\text { Ratio } \\
\text { BPDA:DA* }\end{array}$ & $T_{\text {on }}\left({ }^{\circ} \mathrm{C}\right)$ & $T_{\mathrm{m}}\left({ }^{\circ} \mathrm{C}\right)$ & Pol. T. $\left({ }^{\circ} \mathrm{C}\right)$ & $\begin{array}{l}\text { Theoretical } \\
\text { weight loss }\end{array}$ & $\begin{array}{c}\text { Measured } \\
\text { weight loss }\end{array}$ & Appearance \\
\hline$t-\mathrm{CH}$ & $\mathrm{C}_{10}$ & $1: 1.04$ & 199 & 222 & 190 & $7.1 \%$ & $9.6 \%$ & Transp. brown \\
\hline$t-\mathrm{CH}$ & $\mathrm{C}_{12}$ & $1.01: 1$ & 217 & 234 & 200 & $6.7 \%$ & $7.2 \%$ & Transp. brown \\
\hline$t-\mathrm{CH}$ & $t-\mathrm{CH}$ & $1: 1.01$ & 251 & 266 & 240 & $8.0 \%$ & $18.6 \%$ & Light brown solid \\
\hline
\end{tabular}

\section{Conclusions}

In this work, we report on the synthesis of a series of bis-pyrrolidone dicarboxylic acid (BPDA) monomers obtained from the versatile reaction between the renewable itaconic acid and 1) aliphatic, 2) cycloaliphatic, and 3) aromatic diamines. The monomer synthesis has been studied further, and it was found that itaconic acid can undergo an undesired amidation reaction with amines at higher temperatures, rather than the desired AzaMichael addition reaction. Additionally, the synthesis of dimethyl esters of the BPDA monomers from the reaction of dimethyl itaconate and the abovementioned diamines is reported for the first time. The dimethyl-BPDA monomers have a significantly lower melting point than BPDA monomers, thereby enabling melt-polycondensation conditions without the aid of high boiling solvents during the oligomerization step. Inclusion of 
cycloaliphatic diamines in BPDA monomers resulted in an increase in glass transition temperature in the ensuing BPDA-based polyesters. Furthermore, the poly ([p-Ph-BPDA]$\left.\mathrm{C}_{10-12}\right)$ and poly ([t-CH-BPDA]-C 6 -12) polymers displayed distinct melting behavior, which are the first semi-crystalline homo-polyesters based on BPDA monomers to be reported. Based on their thermal behavior, we consider these polymers promising alternatives to poly-L-lactide. In contrast to amorphous BPDA-based polyesters, semi-crystalline BPDAbased polyesters showed no significant water-absorption after 4 weeks immersion in water and showed limited hydrolysis. Because trans-cyclohexanediamine is currently not available from biomass, the potential biomass content in the poly $\left([t-\mathrm{CH}-\mathrm{BPDA}]-\mathrm{C}_{\mathrm{x}}\right)$ polyester series ranges from $71 \%$ for $\mathrm{C}_{6}$ diol up to $81 \%$ for $\mathrm{C}_{12}$ diol. Additionally, preliminary studies of the use of $t$-CH-BPDA in polyamides rendered amorphous polyamides with aliphatic diamines, though semi-crystalline polyamides can be obtained when using a $t-\mathrm{CH}$ as diamine. Overall, this work shows the potential of the Aza-Michael addition reaction between itaconic acid and diamines as it can be used to develop semicrystalline polymers with a high biobased content.

\section{Conflicts of interest}

There are no conflicts to declare.

\section{Acknowledgements}

This work was performed under the framework of Chemelot InSciTe and is supported by contributions from the European Regional Development Fund (ERDF) within the framework of OP-Zuid and with contributions from the province of Brabant and Limburg and the Dutch Ministry of Economy 


\section{References}

1. Geyer, R., Jambeck, J. R. \& Law, K. L. Production, use, and fate of all plastics ever made. Sci. Adv. 3, e1700782 (2017).

2. Lambert, S. \& Wagner, M. Environmental performance of bio-based and biodegradable plastics: the road ahead. Chem. Soc. Rev. 46, 6855-6871 (2017).

3. Nakajima, H., Dijkstra, P. \& Loos, K. The Recent Developments in Biobased Polymers toward General and Engineering Applications: Polymers that are Upgraded from Biodegradable Polymers, Analogous to Petroleum-Derived Polymers, and Newly Developed. Polymers (Basel). 9, 523 (2017).

4. Zhu, Y., Romain, C. \& Williams, C. K. Sustainable polymers from renewable resources. Nature 540, 354-362 (2016).

5. Iles, A. \& Martin, A. N. Expanding bioplastics production: sustainable business innovation in the chemical industry. J. Clean. Prod. 45, 38-49 (2013).

6. Brockhaus, S., Petersen, M. \& Kersten, W. A crossroads for bioplastics: exploring product developers' challenges to move beyond petroleum-based plastics. J. Clean. Prod. 127, 84-95 (2016).

7. Hillmyer, M. A. The promise of plastics from plants. Science (80-. ). 358, 868-870 (2017).

8. Werpy, T. \& Petersen, G. Top Value Added Chemicals from Biomass: Volume I -- Results of Screening for Potential Candidates from Sugars and Synthesis Gas. Other Information: PBD: 1 Aug 2004 (2004). doi:10.2172/15008859

9. Bozell, J. J. \& Petersen, G. R. Technology development for the production of biobased products from biorefinery carbohydrates - the US Department of Energy's “Top 10" revisited. Green Chem. 12, 539 (2010).

10. Satoh, K. Controlled/living polymerization of renewable vinyl monomers into bio-based polymers. Polym. J. 47, 527536 (2015).

11. Trotta, J. T. et al. Renewable Thermosets and Thermoplastics from Itaconic Acid. ACS Sustain. Chem. Eng. 7, 26912701 (2019).

12. Robert, T. \& Friebel, S. Itaconic acid - a versatile building block for renewable polyesters with enhanced functionality. Green Chem. 18, 2922-2934 (2016).

13. Farmer, T. J., Macquarrie, D. J., Comerford, J. W., Pellis, A. \& Clark, J. H. Insights into post-polymerisation modification of bio-based unsaturated itaconate and fumarate polyesters via aza-michael addition: Understanding the effects of $\mathrm{C}=\mathrm{C}$ isomerisation. J. Polym. Sci. Part A Polym. Chem. 56, 1935-1945 (2018).

14. Teramoto, N., Ozeki, M., Fujiwara, I. \& Shibata, M. Crosslinking and biodegradation of poly(butylene succinate) prepolymers containing itaconic or maleic acid units in the main chain. J. Appl. Polym. Sci. 95, 1473-1480 (2005).

15. Farmer, T. J., Clark, J. H., Macquarrie, D. J., Ogunjobi, J. K. \& Castle, R. L. Post-polymerisation modification of bioderived unsaturated polyester resins via Michael additions of 1,3-dicarbonyls. Polym. Chem. 7, 1650-1658 (2016).

16. Chanda, S. \& Ramakrishnan, S. Poly(alkylene itaconate)s - an interesting class of polyesters with periodically located exo-chain double bonds susceptible to Michael addition. Polym. Chem. 6, 2108-2114 (2015).

17. Winkler, M., Lacerda, T. M., Mack, F. \& Meier, M. A. R. Renewable Polymers from Itaconic Acid by Polycondensation and Ring-Opening-Metathesis Polymerization. Macromolecules 48, 1398-1403 (2015).

18. Giacobazzi, G., Gioia, C., Colonna, M. \& Celli, A. Thia-Michael Reaction for a Thermostable Itaconic-Based Monomer and the Synthesis of Functionalized Biopolyesters. ACS Sustain. Chem. Eng. 7, 5553-5559 (2019).

19. Kumar, S., Krishnan, S., Samal, S. K., Mohanty, S. \& Nayak, S. K. Itaconic acid used as a versatile building block for the synthesis of renewable resource-based resins and polyesters for future prospective: a review. Polym. Int. 66, 13491363 (2017).

20. Paytash, P. L., Sparrow, E. \& Gathe, J. C. The Reaction of Itaconic Acid with Primary Amines. J. Am. Chem. Soc. 72, 1415-1416 (1950).

21. Okada, Y., Banno, T., Toshima, K. \& Matsumura, S. Synthesis and Properties of Polycarboxylate-type Green Surfactants with S- or N-Linkages. J. Oleo Sci. 58, 519-528 (2009).

22. Malferrari, D., Armenise, N., Decesari, S., Galletti, P. \& Tagliavini, E. Surfactants from Itaconic Acid: Physicochemical Properties and Assessment of the Synthetic Strategies. ACS Sustain. Chem. Eng. 3, 1579-1588 (2015).

23. Christian, B. (L'OREAL). FR2961208B1. (2010).

24. Dublanchet, A.-C. (L'Oreal). FR20100054559. (2010).

25. Qi, P., Chen, H.-L., Nguyen, H. T. H., Lin, C.-C. \& Miller, S. A. Synthesis of biorenewable and water-degradable polylactam esters from itaconic acid. Green Chem. 18, 4170-4175 (2016).

26. Wang, R. et al. One-pot synthesis of biodegradable and linear poly(ester amide)s based on renewable resources. J. Appl. Polym. Sci. 133, 43446 (2016).

27. Roy, M., Noordzij, G. J., van den Boomen, Y., Rastogi, S. \& Wilsens, C. H. R. M. Renewable (Bis)pyrrolidone Based Monomers as Components for Thermally Curable and Enzymatically Depolymerizable 2-Oxazoline Thermoset Resins. ACS Sustain. Chem. Eng. 6, 5053-5066 (2018).

28. Avny, Y., Saghian, N. \& Zilkha, A. Thermally Stable Polymers Derived from Itaconic Acid. Isr. J. Chem. 10, 949-957 (1972).

29. Ayadi, F., Mamzed, S., Portella, C. \& Dole, P. Synthesis of bis(pyrrolidone-4-carboxylic acid)-based polyamides derived from renewable itaconic acid-application as a compatibilizer in biopolymer blends. Polym. J. 45, 766-774 (2013).

30. Ali, M. A. et al. Synthesis of High-Performance Biopolyamides Derived from Itaconic Acid and Their Environmental Corrosion. Macromolecules 46, 3719-3725 (2013).

31. Ali, M. A., Tateyama, S. \& Kaneko, T. Synthesis of rigid-rod but degradable biopolyamides from itaconic acid with aromatic diamines. Polym. Degrad. Stab. 109, 367-372 (2014).

32. Wang, Z. et al. Synthesis of fully bio-based polyamides with tunable properties by employing itaconic acid. Polymer (Guildf). 55, 4846-4856 (2014).

33. He, M., Wang, Z., Wang, R., Zhang, L. \& Jia, Q. Preparation of Bio-Based Polyamide Elastomer by Using Green Plasticizers. Polymers (Basel). 8, 257 (2016).

34. Li, G. et al. Industrially scalable and cost-effective synthesis of 1,3-cyclopentanediol with furfuryl alcohol from 
lignocellulose. Green Chem. 18, 3607-3613 (2016).

35. Pingen, D., Diebolt, O. \& Vogt, D. Direct Amination of Bio-Alcohols Using Ammonia. ChemCatChem 5, 2905-2912 (2013)

36. Abraham, R. J. \& Koniotou, R. Conformational analysis. Part 40: a theoretical and NMR investigation of the conformations of cis- and trans-cyclopentane-1,3-diol. Magn. Reson. Chem. 41, 1000-1008 (2003).

37. Noordzij, G. J., Dietz, C. H. J. T., Leoné, N., Wilsens, C. H. R. M. \& Rastogi, S. Small-scale screening of novel biobased monomers: the curious case of 1,3-cyclopentanediol. RSC Adv. 8, 39818-39828 (2018).

38. Zhang, G. et al. Effects of a trans- or cis-cyclohexane unit on the thermal and rheological properties of semi-aromatic polyamides. Polym. Chem. 7, 44-53 (2016).

39. Kricheldorf, H. R. \& Schwarz, G. Cis / trans isomerism of 1, 4-cyclohexanedicarboxylic acid in crystalline , liquidcrystalline and amorphous polyesters. Macromol. Chem. Phys. 188, 1281-1294 (1987).

40. Berti, C. et al. Environmentally Friendly Copolyesters Containing 1,4-Cyclohexane Dicarboxylate Units, 1-Relationships Between Chemical Structure and Thermal Properties. Macromol. Chem. Phys. 211, 1559-1571 (2010).

41. Colonna, M. et al. Poly(1,4-cyclohexylenedimethylene-1, 4-cyclohexanedicarboxylate): analysis of parameters affecting polymerization and cis-trans isomerization. Polym. Int. 60, 1607-1613 (2011).

42. Wang, J. et al. Modification of poly(ethylene 2,5-furandicarboxylate) (PEF) with 1, 4-cyclohexanedimethanol: Influence of stereochemistry of 1,4-cyclohexylene units. Polymer (Guildf). 137, 173-185 (2018).

43. Saeidlou, S., Huneault, M. A., Li, H. \& Park, C. B. Poly(lactic acid) crystallization. Prog. Polym. Sci. 37, 1657-1677 (2012). 


\section{Supplementary information for chapter 5}

Due to page restrictions only the most relevant synthesis and analysis details are given. Full supplementary information can be found online at:

https://doi.org/10.1039/C9PY00463G.

\section{Monomer Synthesis}

The obtained BPDA monomers are abbreviated to xx-BPDA where $x x$ denotes the type of diamine used: e.g. BPDA with 1,8-octanediamine is abbreviated as $\mathrm{C}_{8}$-BPDA, BPDA with trans-1,4-cyclohexanediamine is abbreviated as $t$-CH-BPDA. The synthesis to obtain BPDA monomers is divided in two synthesis pathways. Either via itaconic acid (Scheme 5-2, $\mathrm{R}=\mathrm{H}$ ), or via dimethyl itaconate (Scheme 5-2, $\mathrm{R}=\mathrm{CH}_{3}$ ) to obtain BPDA monomers with free acid, or dimethyl-ester functionality respectively. The only exception is the synthesis of $p$-Ph-BPDA-dm, which was obtained via methylation of $p$-Ph-BPDA.

\section{Synthesis of t-CP-BPDA-dm}

Dimethyl itaconate $(8.98 \mathrm{~g}, 56.8 \mathrm{mmol})$ and trans-1,3-cyclopentanediamine $(2.74 \mathrm{~g}, 27.4 \mathrm{mmol})$ were dissolved in $30 \mathrm{~mL}$ of methanol in a $100 \mathrm{~mL}$ round-bottom flask, equipped with a magnetic stirring bar. The reaction mixture was stirred at reflux overnight to yield a viscous orange oil. The product was isolated after column chromatography $\left.\mathrm{MeOH}: \mathrm{CHCl}_{3} 3: 97 \mathrm{v} \%\right)$ as a yellow oil. $\left(5.12 \mathrm{~g}, 53\right.$ yield). ${ }^{1} \mathrm{H} \mathrm{NMR}\left(\mathrm{CDCl}_{3}, 300\right.$ $\mathrm{MHz})$ : $\delta 3.97(\mathrm{~m} \mathrm{2.0H}), 3.75(\mathrm{~s}, 6.01 \mathrm{H}), 3.54(\mathrm{~m}, 4.38 \mathrm{H}), 3.22(\mathrm{p}, 2.06 \mathrm{H}), 2.68(\mathrm{~m}, 4.29 \mathrm{H}), 1.80(4.22 \mathrm{H})$, $1.59(\mathrm{~m}, 4.21 \mathrm{H}) .{ }^{13} \mathrm{C} \mathrm{NMR}\left(\mathrm{CDCl}_{3}, 300 \mathrm{MHz}\right): \delta 173.1,172.2,52.5,50.4,44.8,36.0,34.5,30.6,28.2$.

\section{Synthesis of $\boldsymbol{c}$-CP-BPDA-dm}

Dimethyl itaconate $(9.84 \mathrm{~g}, 60 \mathrm{mmol})$ and cis-1,3-cyclopentanediamine $(2.87,28.7 \mathrm{mmol})$ were dissolved in $30 \mathrm{~mL}$ of methanol in a $100 \mathrm{~mL}$ round-bottom flask, equipped with a magnetic stirring bar. The reaction mixture was stirred at reflux overnight to yield a viscous orange oil. The product was isolated after column chromatography ( $\left.\mathrm{MeOH}: \mathrm{CHCl}_{3} 3: 97 \mathrm{v} \%\right)$ as a yellow oil. $\left(5.59 \mathrm{~g}, 55\right.$ yield). ${ }^{1} \mathrm{H} \mathrm{NMR}\left(\mathrm{CDCl}_{3}, 300 \mathrm{MHz}\right): \delta$ $4.66(\mathrm{p}, 1.86 \mathrm{H}), 3.75(\mathrm{~s}, 6.0 \mathrm{H}), 3.60(\mathrm{~d}, 3.95 \mathrm{H}), 3.23(\mathrm{p}, 1.97 \mathrm{H}), 2.70(\mathrm{~m}, 4.0 \mathrm{H}), 2.0(1.90 \mathrm{H}), 1.86(\mathrm{~m}$, $2.60 \mathrm{H}), 1.61(\mathrm{~m}, 1.78 \mathrm{H}) .{ }^{13} \mathrm{C} \mathrm{NMR}\left(\mathrm{CDCl}_{3}, 300 \mathrm{MHz}\right): \delta 173.2,172.2,52.5,50.8,45.5,36.1,34.7,31.0$, 26.9.

\section{Synthesis of $t$-CH-BPDA-dm}

Dimethyl itaconate $(79.6 \mathrm{~g}, 0.5 \mathrm{~mol})$ and trans-1,4-cyclohexanediamine $(25 \mathrm{~g}, 0.22 \mathrm{mmol})$ were dissolved in $30 \mathrm{~mL}$ of methanol in a $250 \mathrm{~mL}$ round-bottom flask, equipped with a magnetic stirring bar. The reaction mixture was stirred at reflux overnight, and the product could directly be obtained as white crystals after cooling of the reaction mixture $\left(72.5 \mathrm{~g}, 90 \%\right.$ yield). ${ }^{1} \mathrm{H} \mathrm{NMR}\left(\mathrm{CDCl}_{3}, 300 \mathrm{MHz}\right): \delta 3.97(\mathrm{~m}, 2.0 \mathrm{H}), 3.75(\mathrm{~s}$, $6.01 \mathrm{H}), 3.54(\mathrm{~m}, 4.38 \mathrm{H}), 3.22(\mathrm{p}, 2.06 \mathrm{H}), 2.68(\mathrm{~m}, 4.29 \mathrm{H}), 1.80(\mathrm{~m}, 4.22 \mathrm{H}), 1.59(\mathrm{~m}, 4.21 \mathrm{H}) .{ }^{13} \mathrm{C} \mathrm{NMR}$ $\left(\mathrm{CDCl}_{3}, 300 \mathrm{MHz}\right): \delta$ 173.2, 172.0, 52.5, 49.4, 44.9, 36.2, 34.6, 28.4.

\section{Synthesis of $c$-CH-BPDA-dm}

Dimethyl itaconate $(9.31 \mathrm{~g}, 2.18 \mathrm{mmol})$ and cis-1,4-cyclohexanediamine $(3.09 \mathrm{~g}, 27.05 \mathrm{mmol})$ were dissolved in $30 \mathrm{~mL}$ of methanol in a $100 \mathrm{~mL}$ round-bottom flask, equipped with a magnetic stirring bar. The reaction mixture was stirred at reflux overnight to yield a viscous orange oil. The product was isolated after column chromatography ( $\mathrm{MeOH}: \mathrm{CHCl}_{3} 1: 19 \mathrm{v} \%$, RF 0.48 ) as a yellow oil. (5.73 g, $63 \%$ yield). ${ }^{1} \mathrm{H}$ NMR $\left(\mathrm{CDCl}_{3}, 300 \mathrm{MHz}\right): \delta 3.96(\mathrm{~m}, 2.0 \mathrm{H}), 3.69(\mathrm{~s}, 6.0 \mathrm{H}), 3.65(\mathrm{~m}, 3.84 \mathrm{H}), 3.16(\mathrm{p}, 2.0 \mathrm{H}), 2.63(\mathrm{~d}, 3.98 \mathrm{H}), 1.82$ $(\mathrm{m}, 3.95 \mathrm{H}), 1.64(\mathrm{~m}, 4.16 \mathrm{H}) .{ }^{13} \mathrm{C} \mathrm{NMR}\left(\mathrm{CDCl}_{3}, 300 \mathrm{MHz}\right): \delta$ 173.3, 172.3, 52.5, 48.1, 47.3, 36.4, 34.5, 30.0. 


\section{Synthesis of $p$-Ph-BPDA}

Itaconic acid $(7.83 \mathrm{~g}, 60.2 \mathrm{mmol})$ and para-phenylenediamine $(3.25 \mathrm{~g}, 30.1 \mathrm{mmol})$ were loaded in a 100 $\mathrm{mL}$ round-bottom flask. The reaction mixture was stirred overnight at $130{ }^{\circ} \mathrm{C}$. Recrystallization from boiling water yielded the product as white solids $\left(11.5 \mathrm{~g}, 99 \%\right.$ yield). ${ }^{1} \mathrm{H} \mathrm{NMR}\left(\mathrm{CDCl}_{3}, 300 \mathrm{MHz}\right): \delta 7.55(\mathrm{~s}, 4.0 \mathrm{H})$, $4.26(\mathrm{~m}, 4.04 \mathrm{H}), 3.60(\mathrm{p}, 2.09 \mathrm{H}), 3.20(\mathrm{~d}, 4.0 \mathrm{H}) .{ }^{13} \mathrm{C} \mathrm{NMR}\left(\mathrm{CDCl}_{3}, 300 \mathrm{MHz}\right): \delta 178.3,162.5,161.9,161.3$, $160.7,135.4,123.4,51.7,35.7,34.6$

\section{Synthesis of $p$-Ph-BPDA-dm}

P-Ph-BPDA (5.1 g, $15.3 \mathrm{mmol}$ ) was suspended in $100 \mathrm{~mL}$ of methanol with $2 \mathrm{~mL}$ of sulfuric acid in a 250 $\mathrm{mL}$ round-bottom flask. The suspension was refluxed for 72 hours, the methanol and formed water were removed in vacuo, the obtained product was re-suspended in $100 \mathrm{~mL}$ of methanol and refluxed for another $18 \mathrm{hr}$. The suspension was a grey slurry in deep purple liquid. After cooling, the product could be isolated as a grey solid by filtration $\left(4.45 \mathrm{~g}, 81 \%\right.$ yield). ${ }^{1} \mathrm{H} \mathrm{NMR}\left(\mathrm{CDCl}_{3}, 300 \mathrm{MHz}\right): \delta 7.54(\mathrm{~s}, 4.0 \mathrm{H}), 3.99(\mathrm{~m}$, $4.15 \mathrm{H}), 3.72(\mathrm{~s}, 5.97 \mathrm{H}), 3.31(\mathrm{p}, 2.16 \mathrm{H}), 2.84(\mathrm{~m}, 4.19 \mathrm{H})$.

\section{Synthesis of $p$-Xy-BPDA-dm}

Dimethyl itaconate $(20.4 \mathrm{~g}, 128.7 \mathrm{mmol})$ and para-xylylenediamine $(8.8 \mathrm{~g}, 61.3 \mathrm{mmol})$ were dissolved in $30 \mathrm{~mL}$ of methanol in a $100 \mathrm{~mL}$ round-bottom flask, equipped with a magnetic stirring bar. The reaction mixture was stirred at reflux overnight, and the product could directly be obtained as white crystals after cooling of the reaction mixture $\left(17.5 \mathrm{gr}, 74 \%\right.$ yield). ${ }^{1} \mathrm{H} \mathrm{NMR}\left(\mathrm{CDCl}_{3}, 300 \mathrm{MHz}\right): \delta 7.22(2,4.0 \mathrm{H}), 4.45(\mathrm{~m}$, 4.07H), $3.73(\mathrm{~s}, 6.0 \mathrm{H}), 3.48(\mathrm{~m}, 3.83 \mathrm{H}), 3.23(\mathrm{p}, 2.03 \mathrm{H}), 2.76(\mathrm{~m}, 4.08 \mathrm{H})$. 


\section{NMR analysis of monomers}

\section{NMR analysis of $t$-CP-BPDA-dm}

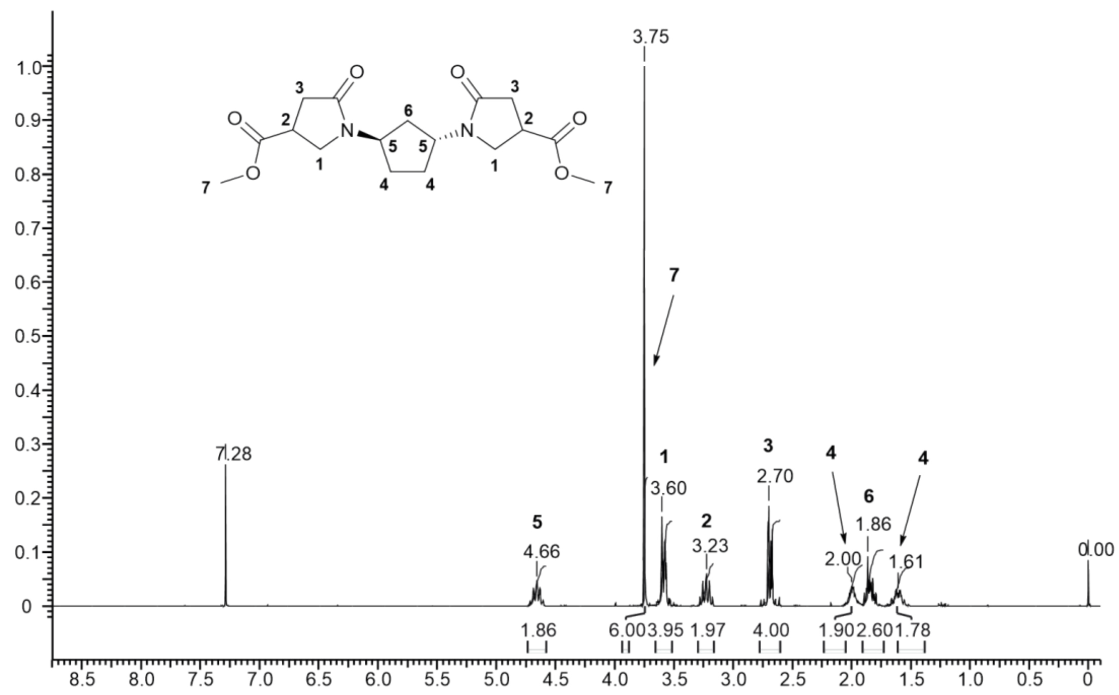

Figure $S 5-1$. ${ }^{1} \mathrm{H}$-NMR of $t$-CP-BPDA-dm.

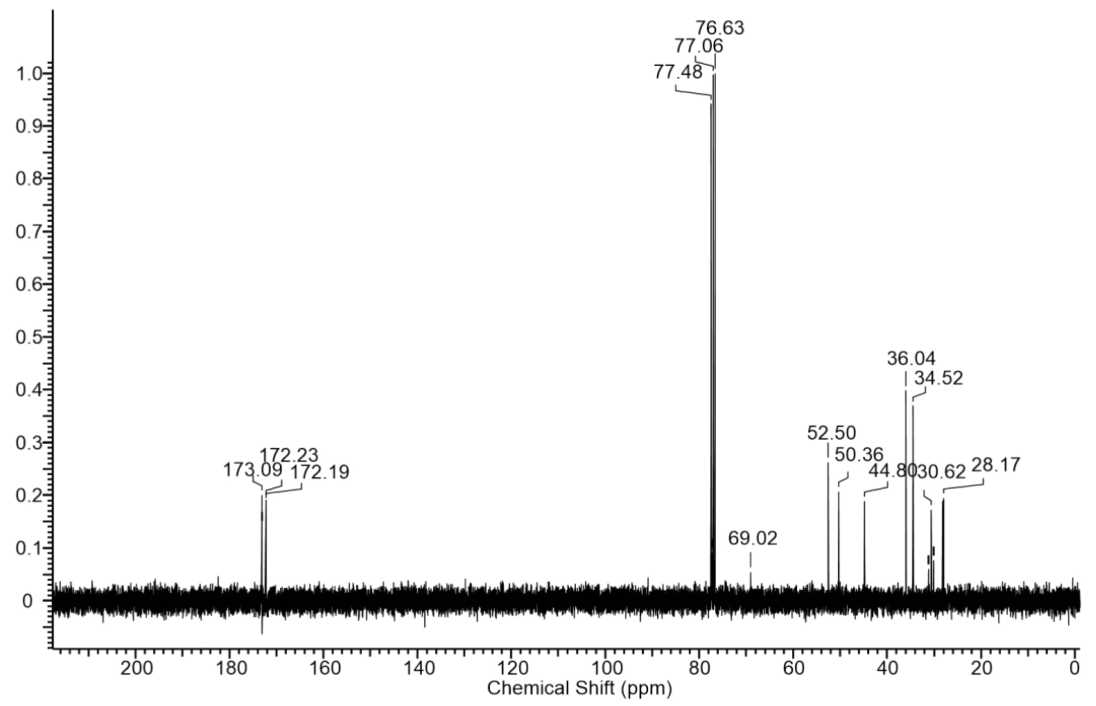

Figure S5 - 2. ${ }^{13} \mathrm{C}-\mathrm{NMR}$ of $t$-CP-BPDA-dm. 


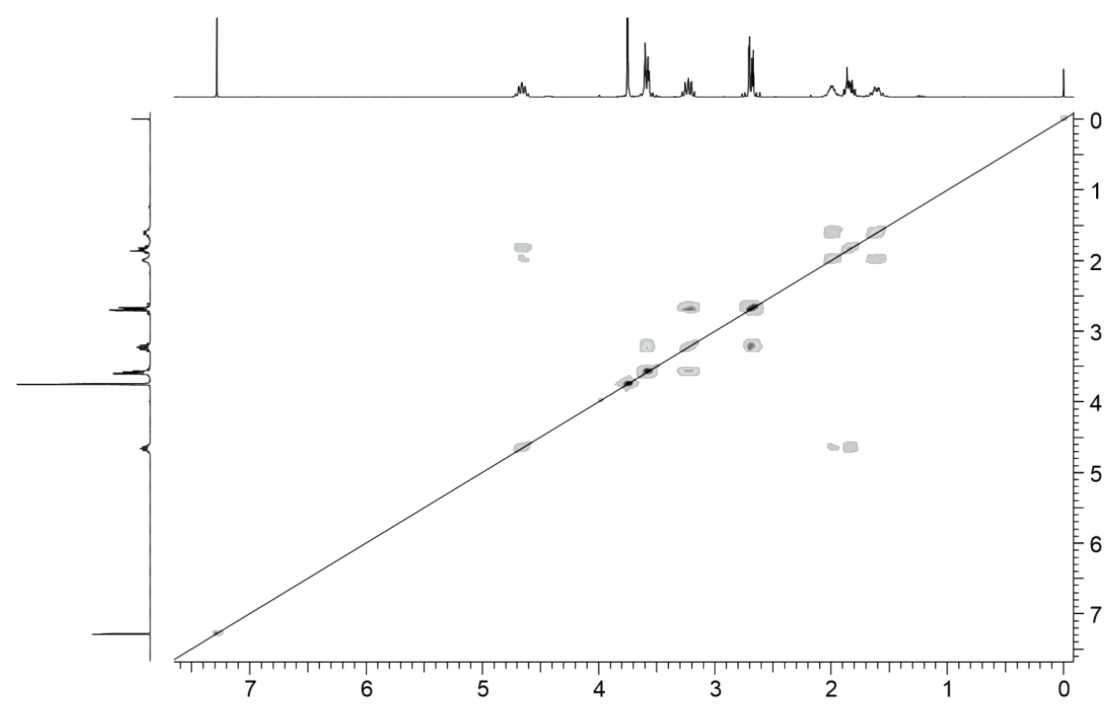

Figure $55-3$. COSY $\left({ }^{1} \mathrm{H}-{ }^{-1} \mathrm{H}\right)$ NMR of $t$-CP-BPDA-dm.

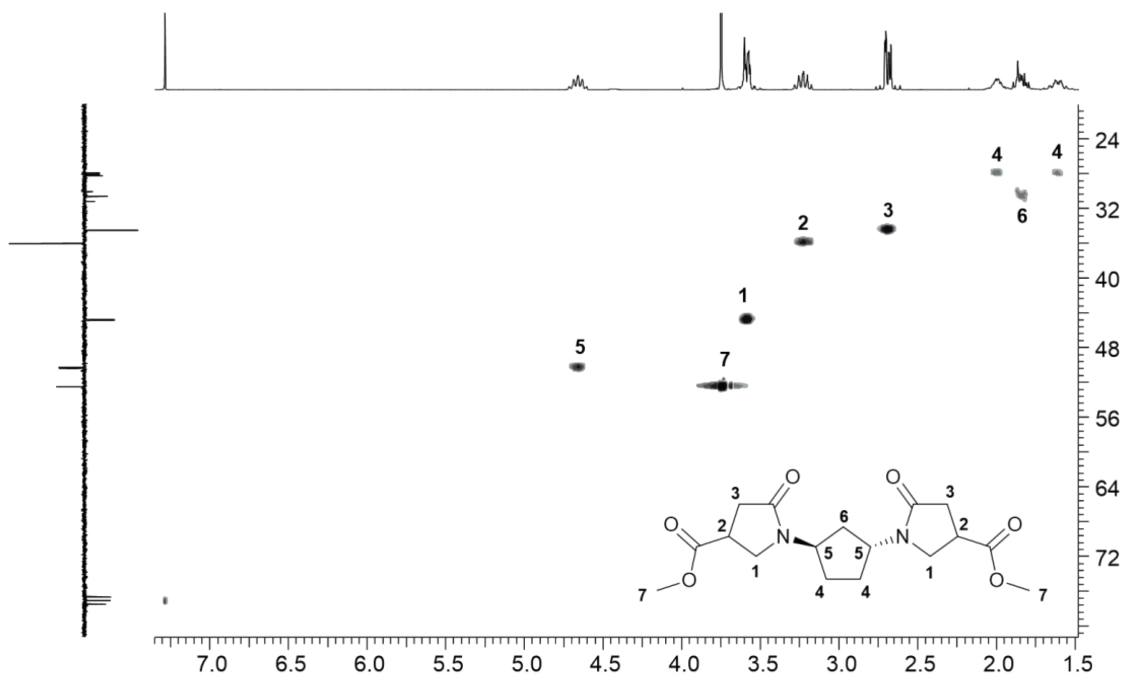

Figure $S 5$ - 4. HSQC $\left({ }^{1} \mathrm{H}^{-13} \mathrm{C}\right.$ APT $)$ NMR of $t$-CP-BPDA-dm. 
NMR analysis of c-CP-BPDA-dm

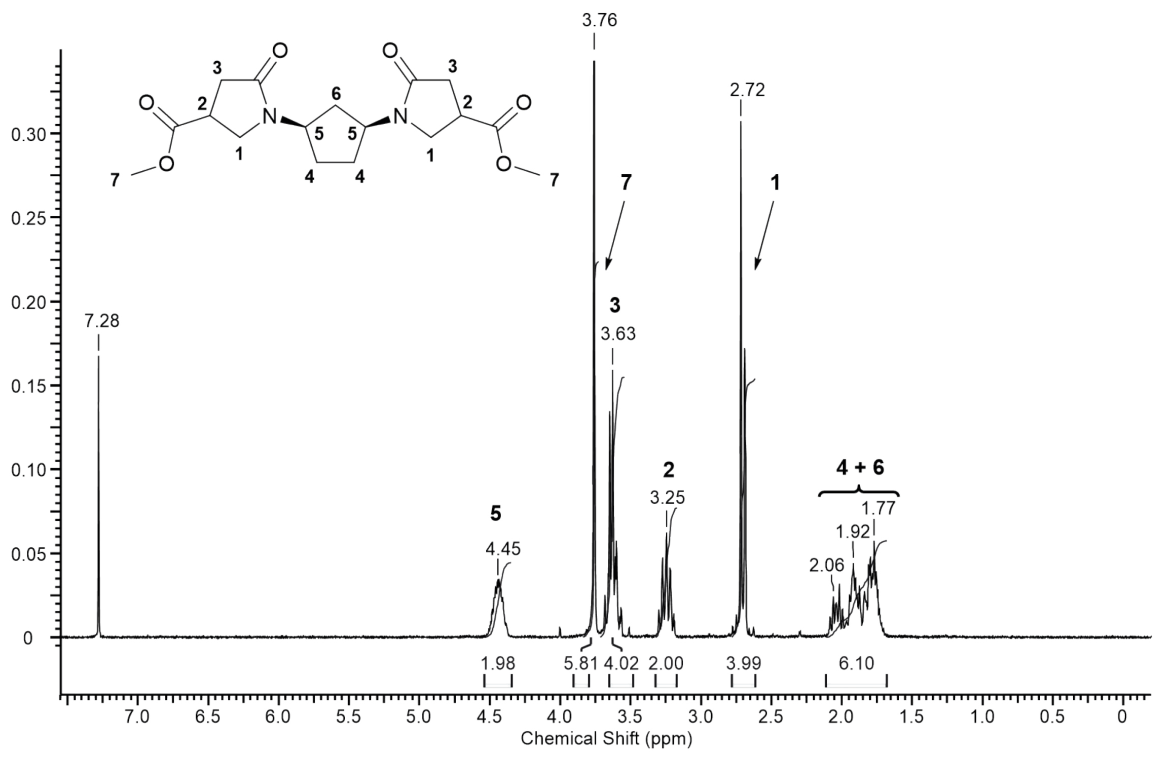

Figure S5 - 5. ${ }^{1} \mathrm{H}-\mathrm{NMR}$ of $c$-CP-BPDA-dm.

\section{NMR analysis of $t$-CH-BPDA-dm}

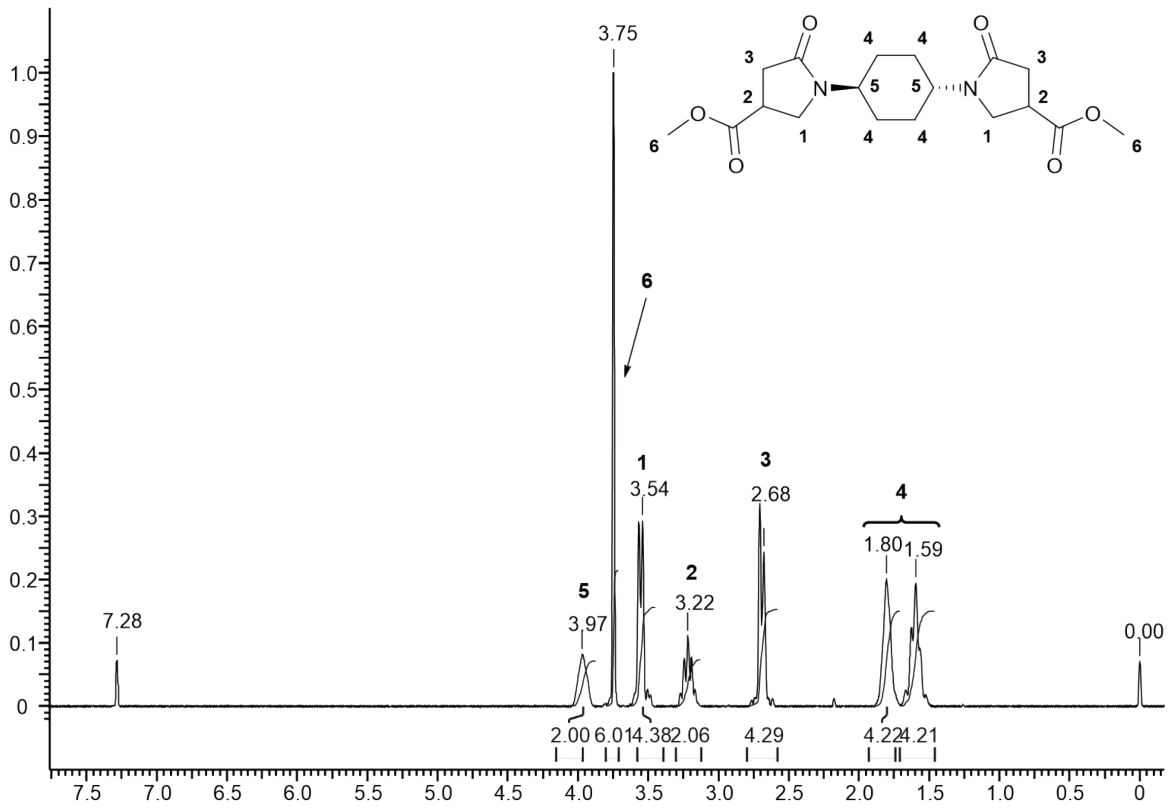

Figure S5 - 6. ${ }^{1} \mathrm{H}-\mathrm{NMR}$ of $t$-CH-BPDA-dm. 


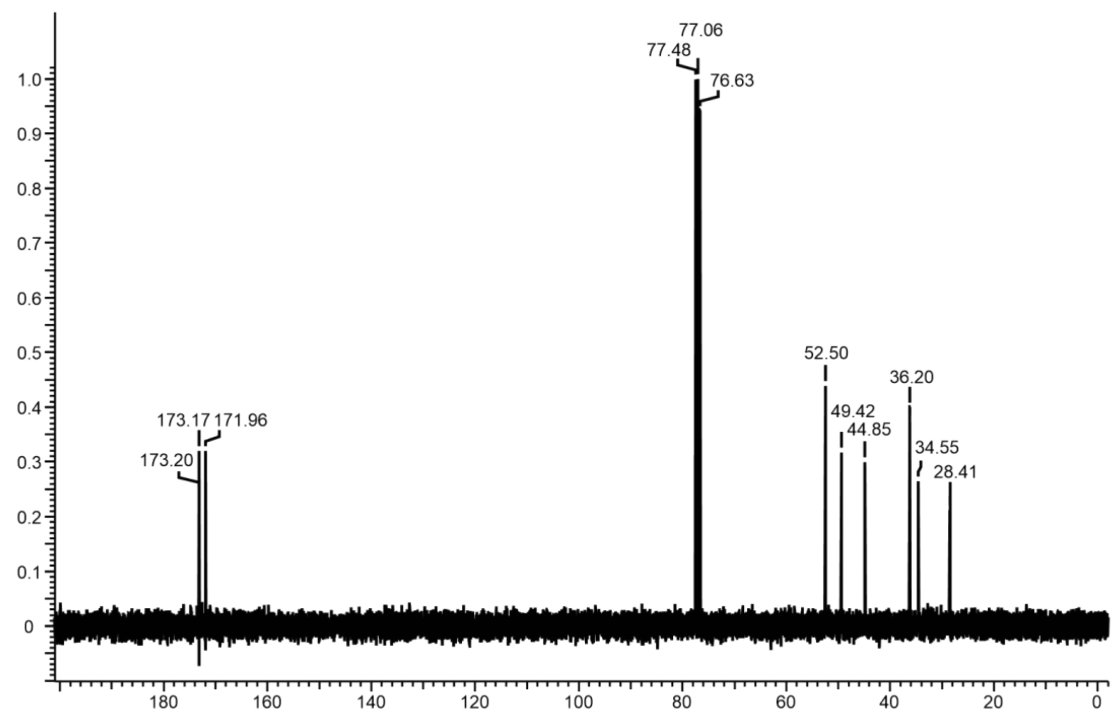

Figure S5 - 7. ${ }^{13} \mathrm{C}-\mathrm{NMR}$ of $t$-CH-BPDA-dm.

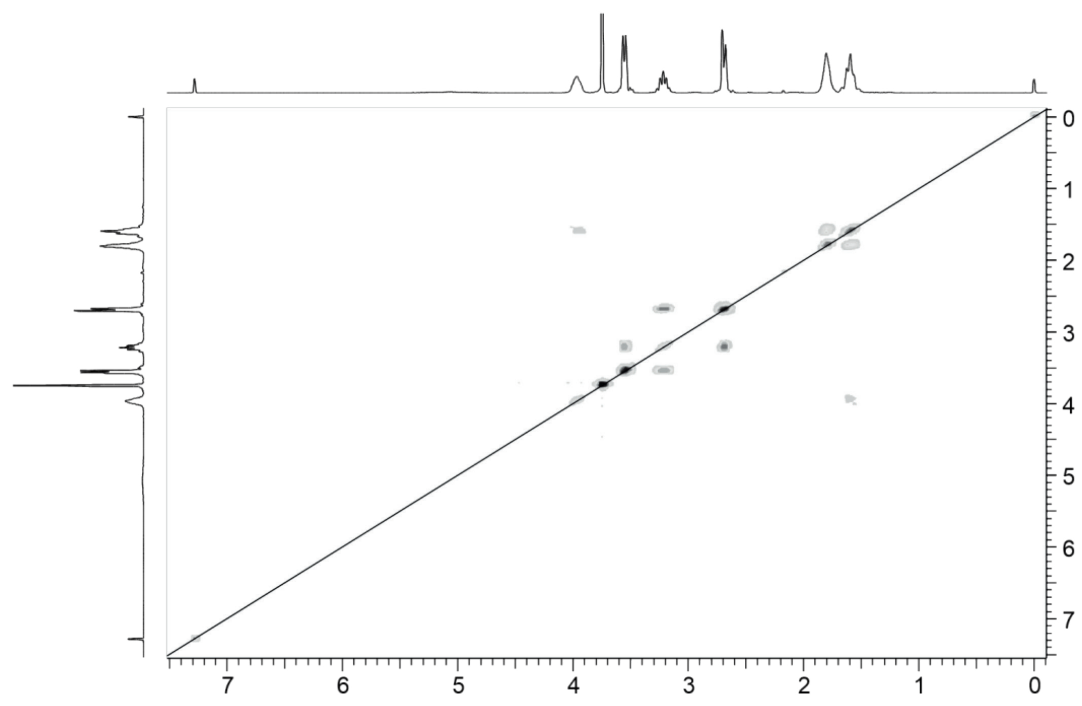

Figure $S 5-8$. COSY $\left({ }^{1} \mathrm{H}-{ }^{1} \mathrm{H}\right)$ NMR of $t$-CH-BPDA-dm. 


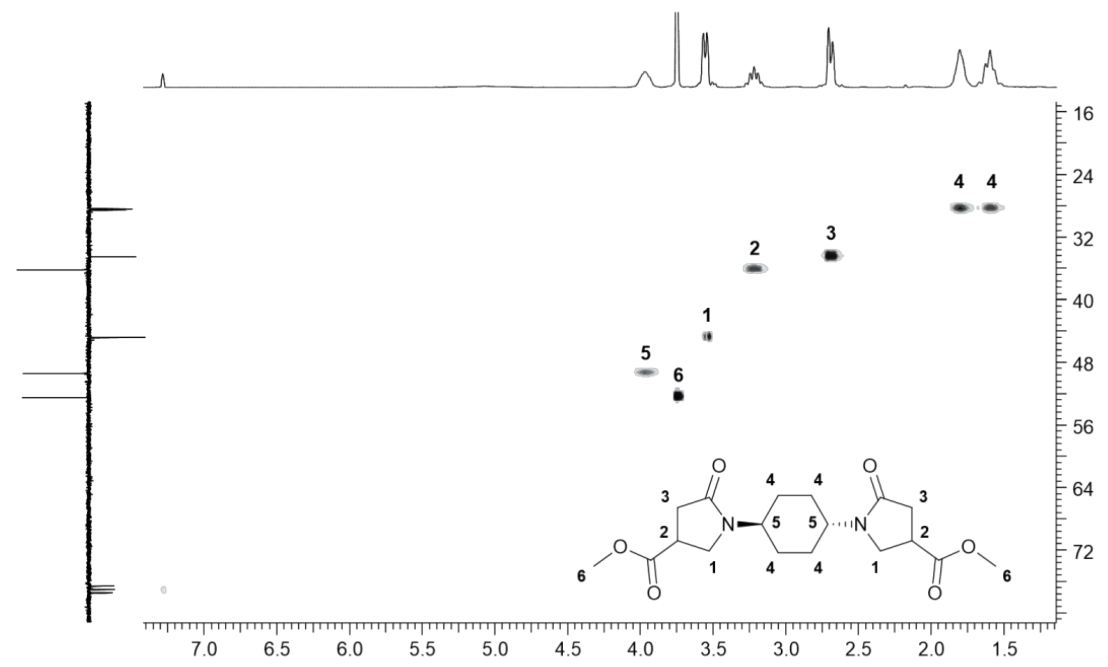

Figure S5 - 9. HSQC $\left({ }^{1} \mathrm{H}-{ }^{13} \mathrm{C}\right.$ APT) NMR of $t$-CH-BPDA-dm.

\section{NMR analysis of $c-C H-B P D A-d m$}

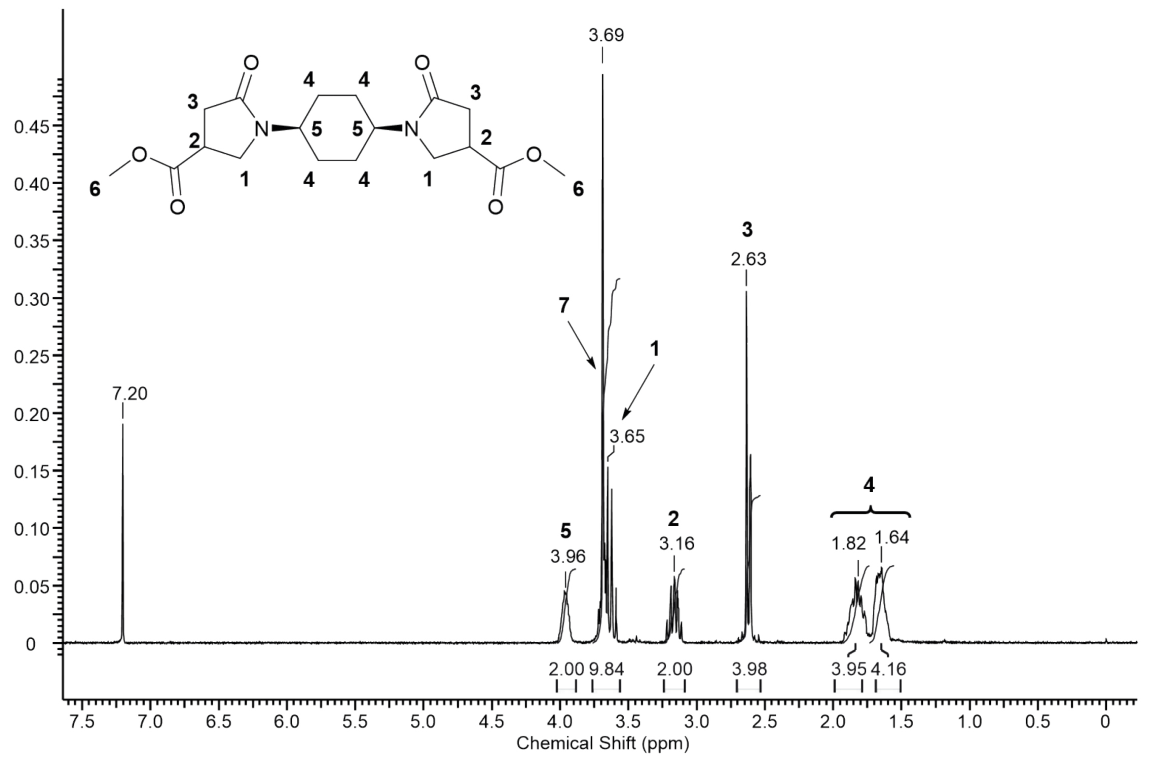

Figure S5 - 10. ${ }^{1} \mathrm{H}-\mathrm{NMR}$ of $c-\mathrm{CH}-\mathrm{BPDA}-\mathrm{dm}$. 
NMR analysis of $p-P h-B P D A$

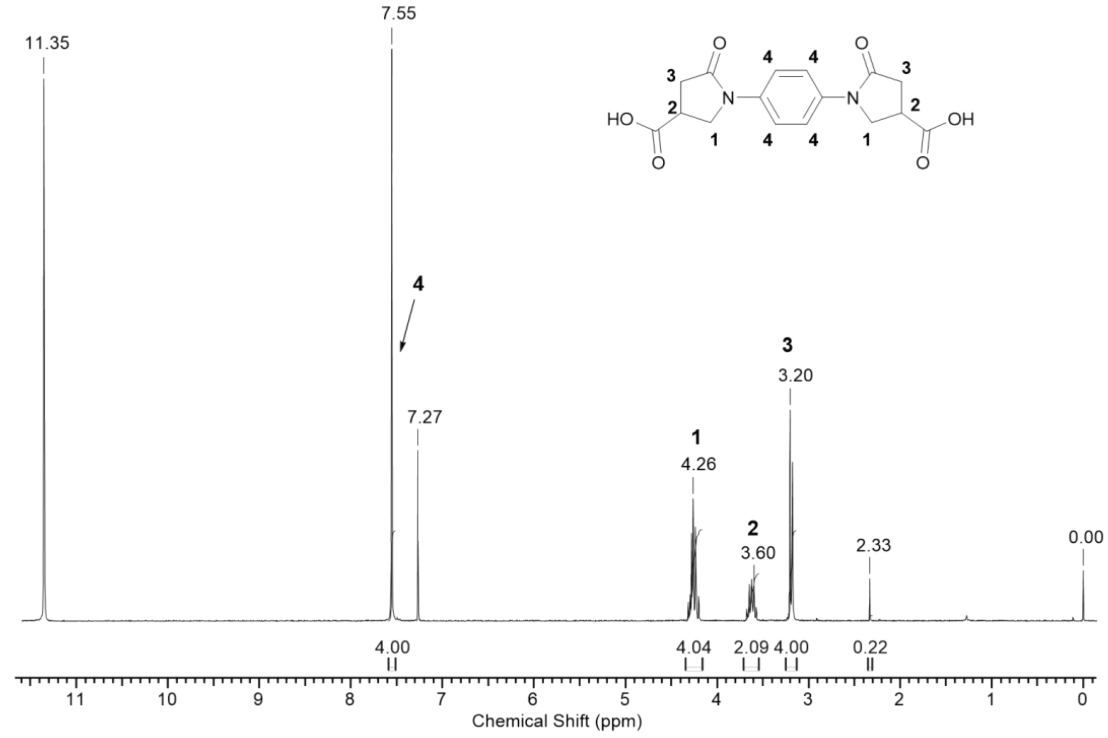

Figure $\mathrm{S} 5-11 .{ }^{1} \mathrm{H}-\mathrm{NMR}$ of $p$-Ph-BPDA.

\section{NMR analysis of $p$-Ph-BPDA-dm}

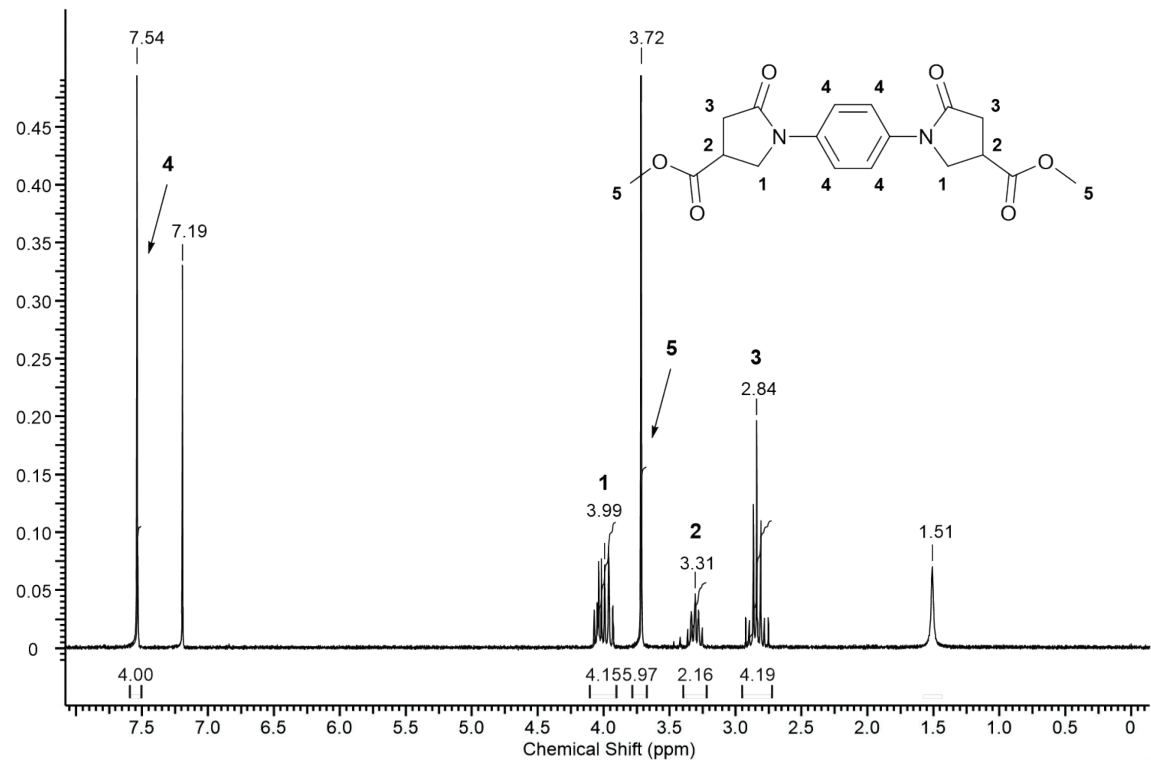

Figure $\mathrm{S} 5-12 .{ }^{1} \mathrm{H}-\mathrm{NMR}$ of $p$-Ph-BPDA-dm. 


\section{NMR analysis of $p-X y-B P D A-d m$}

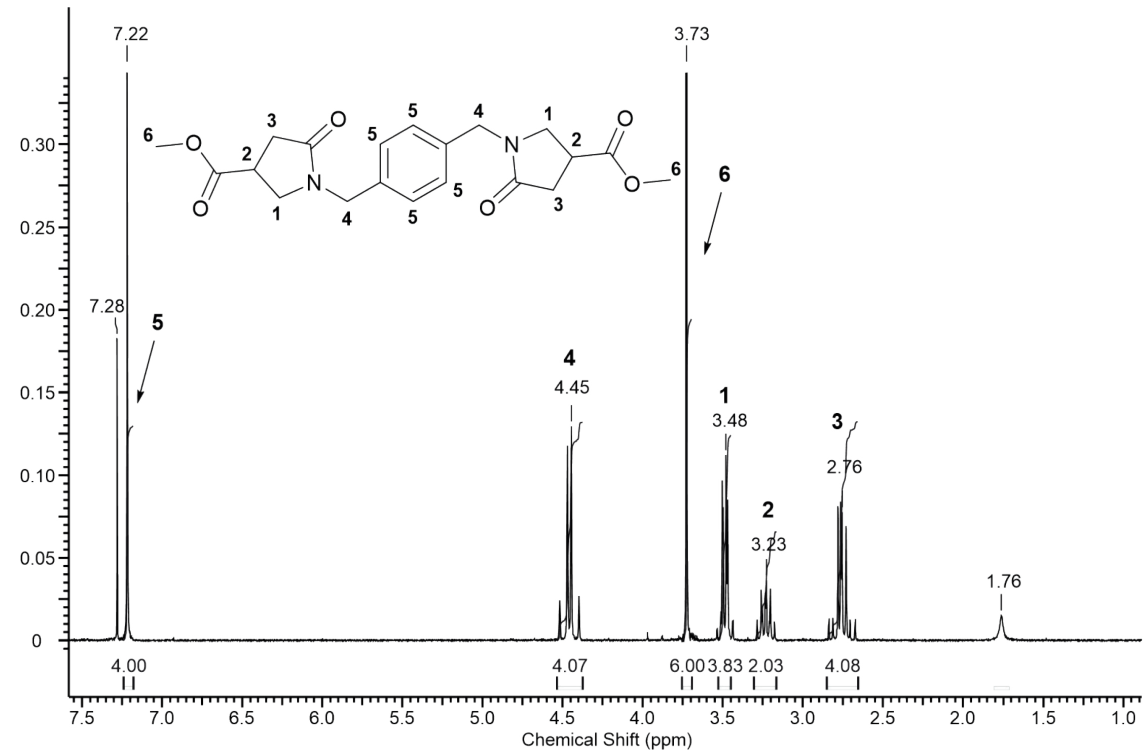

Figure S5 - 13. ${ }^{1} \mathrm{H}-\mathrm{NMR}$ of $p$-Xy-BPDA-dm.

\section{TGA analysis of monomers}

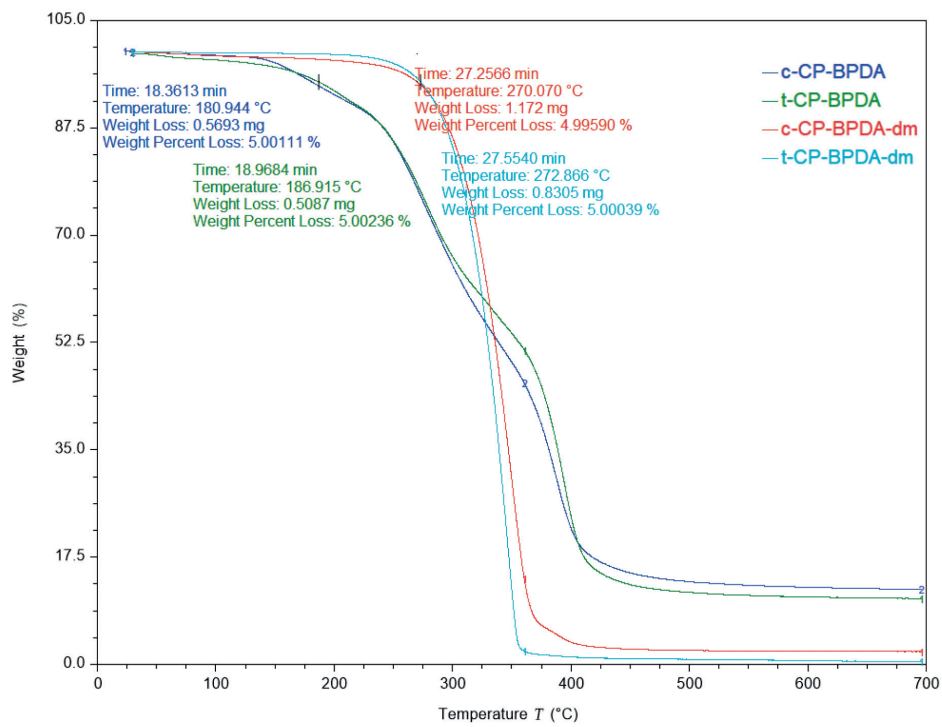

Figure S5 - 14. TGA of monomers c-CP-BPDA (light blue), $c$-CP-BPDA-dm (green), $t$-CP-BPDA (red), $t$-CP-BPDA-dm (blue). 


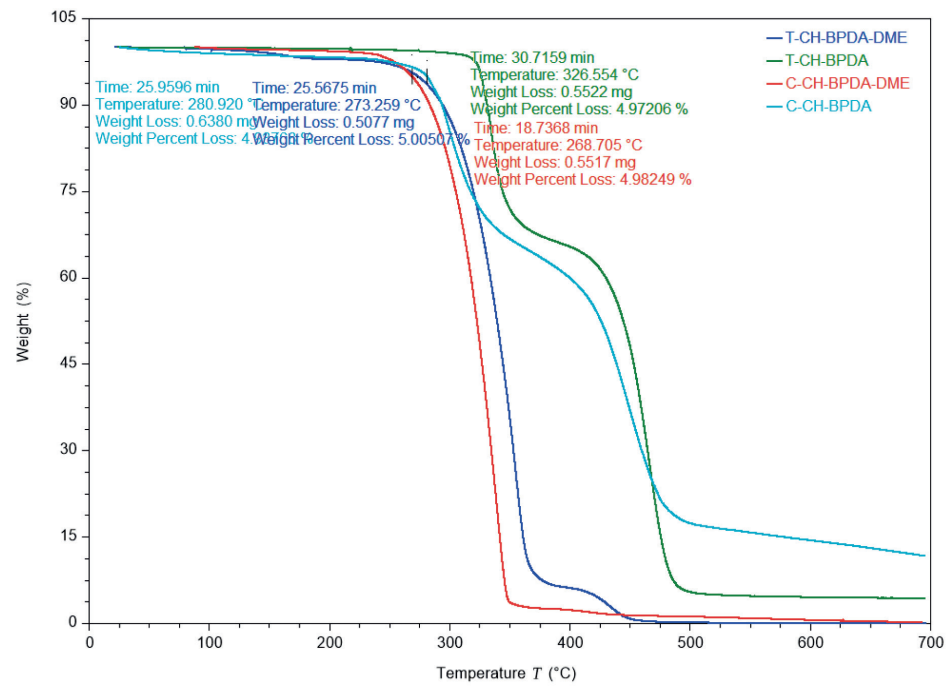

Figure S5 - 15. TGA of monomers $c-\mathrm{CH}-\mathrm{BPDA}$ (light blue), $c-\mathrm{CH}-\mathrm{BPDA}-\mathrm{dm}$ (red), $t$-CH-BPDA (green), $t$-CH-BPDA-dm (blue).

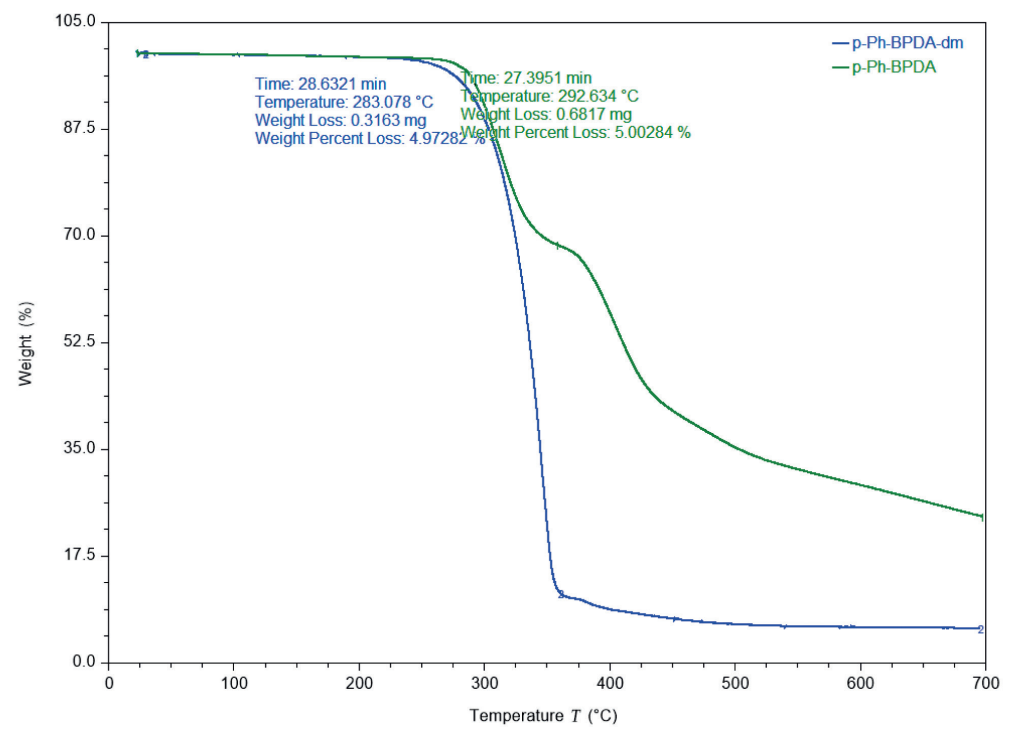

Figure S5 - 16. TGA of monomers $p$-Ph-BPDA (green) and p-Ph-BPDA-dm. 


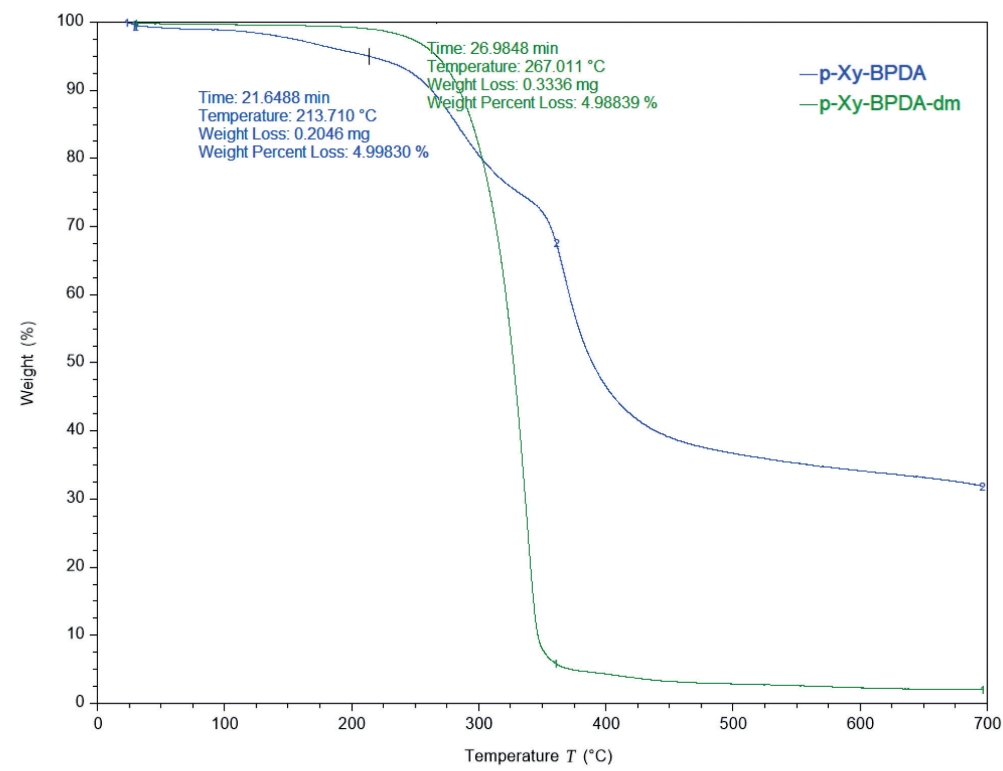

Figure S5 - 17. TGA of monomers $p$-Xy-BPDA (blue) and $p$-Xy-BPDA-dm (green).

\section{NMR analysis of organic BPDA-diamine salts}

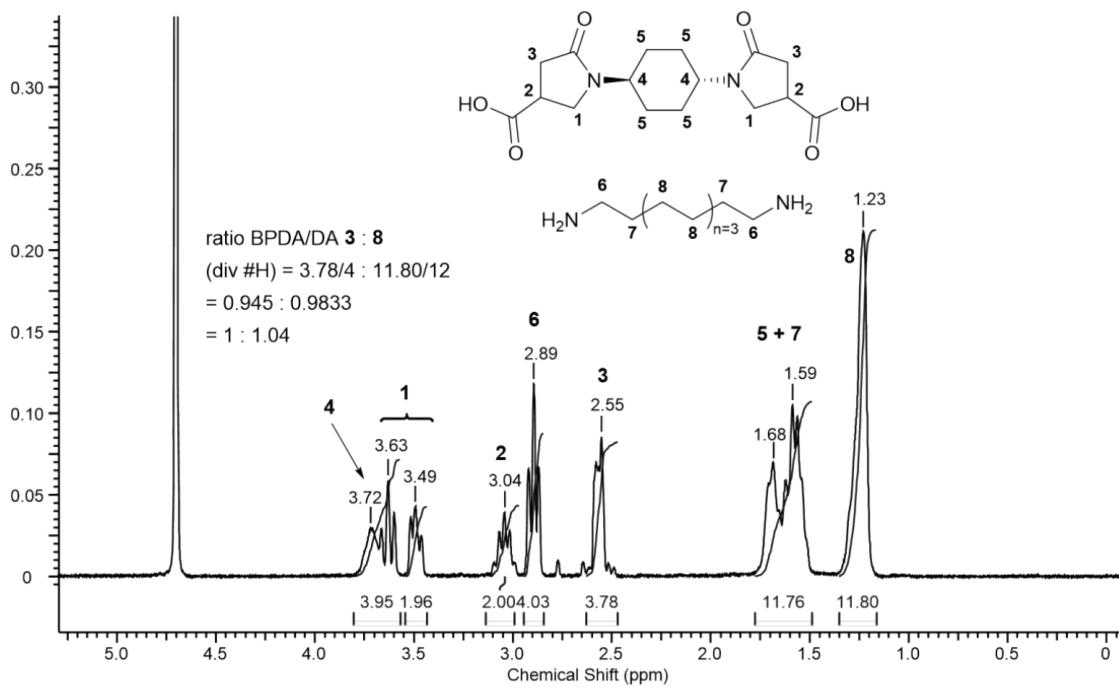

Figure S5 - 18. ${ }^{1} \mathrm{H}-\mathrm{NMR}$ analysis of organic salt of $t$-CH-BPDA and 1,10-decanediamine. 


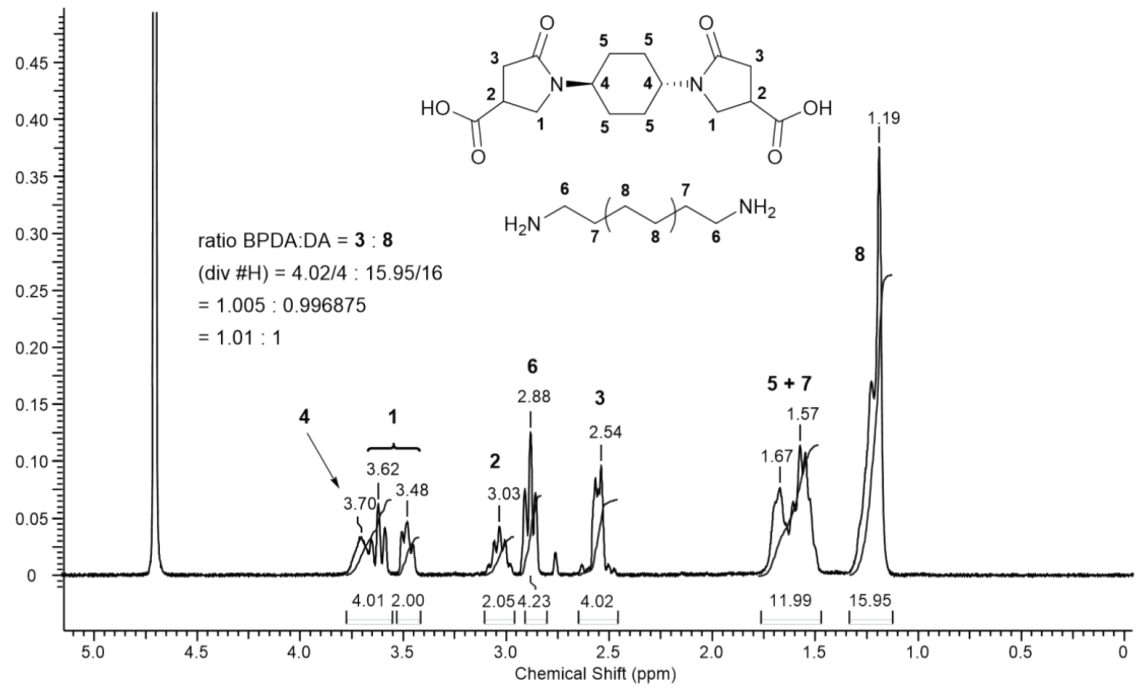

Figure S5 - 19. ${ }^{1} \mathrm{H}-\mathrm{NMR}$ analysis of organic salt of $t$-CH-BPDA and 1,12-dodecanediamine.

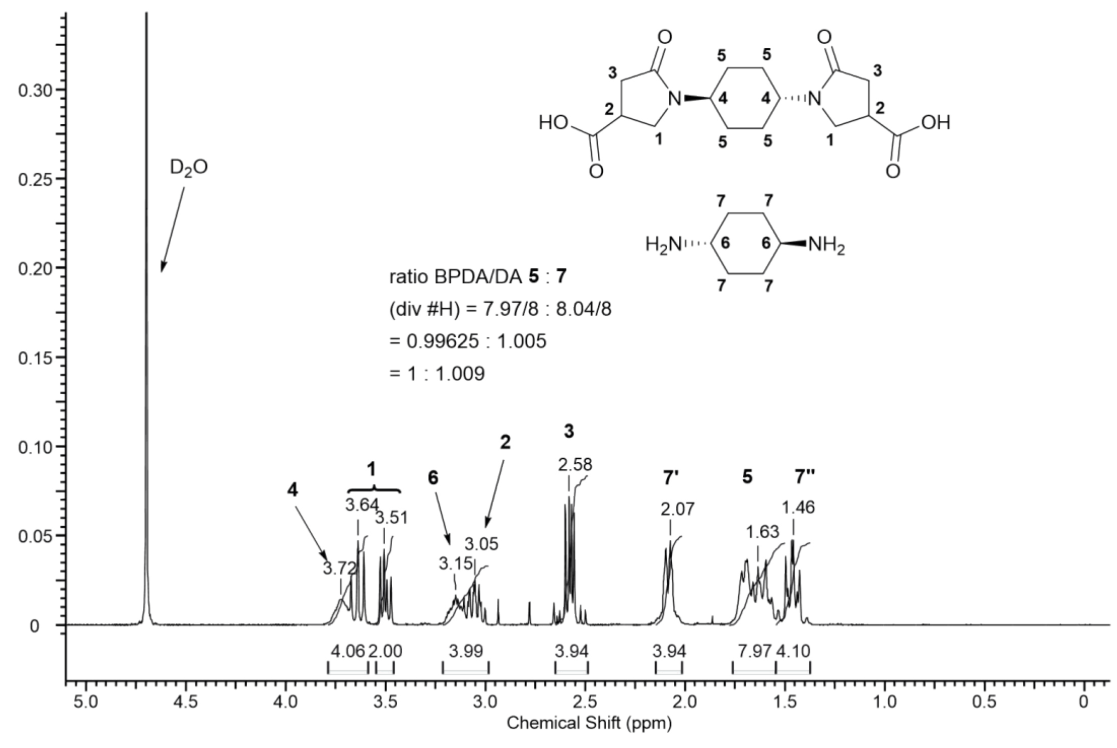

Figure S5 - 20. ${ }^{1} \mathrm{H}-\mathrm{NMR}$ analysis of organic salt of $t$-CH-BPDA and $t$-CHdiamine. 


\section{TGA analysis of organic BPDA-diamine salts}

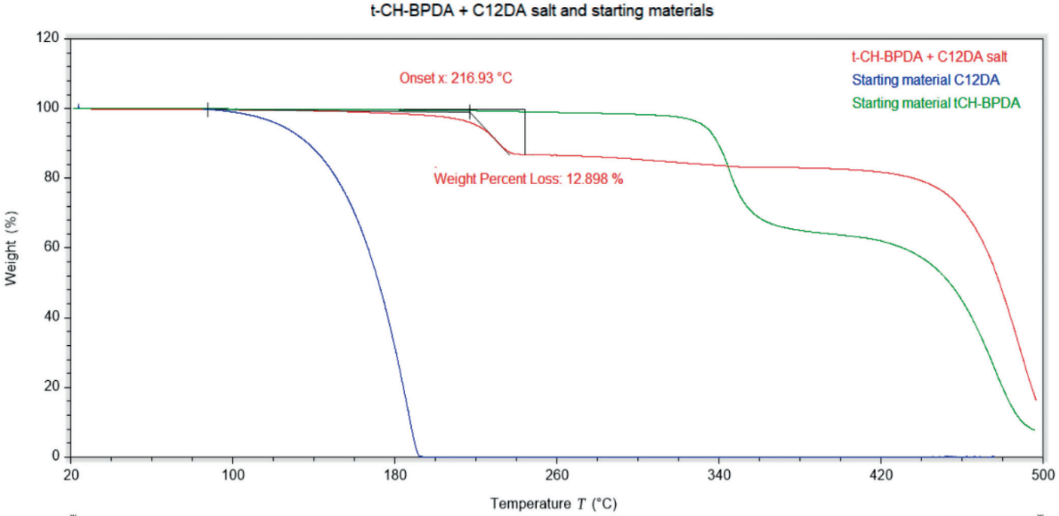

Figure S5 - 21. TGA analysis of starting materials 1,12-dodecanediamine (blue), $t$-CH-BPDA (green), and the organic salt of $t$-CH-BPDA with 1,12-dodecanediamine (red). Visible is the evaporation of 1,12dodecanediamine above $100{ }^{\circ} \mathrm{C}$, thermal decarboxylation of $t$ - $\mathrm{CH}$-BPDA above $320{ }^{\circ} \mathrm{C}$, and the change in thermal stability of the organic salt when heated.

\section{TGA analysis of BPDA-based polyamides}

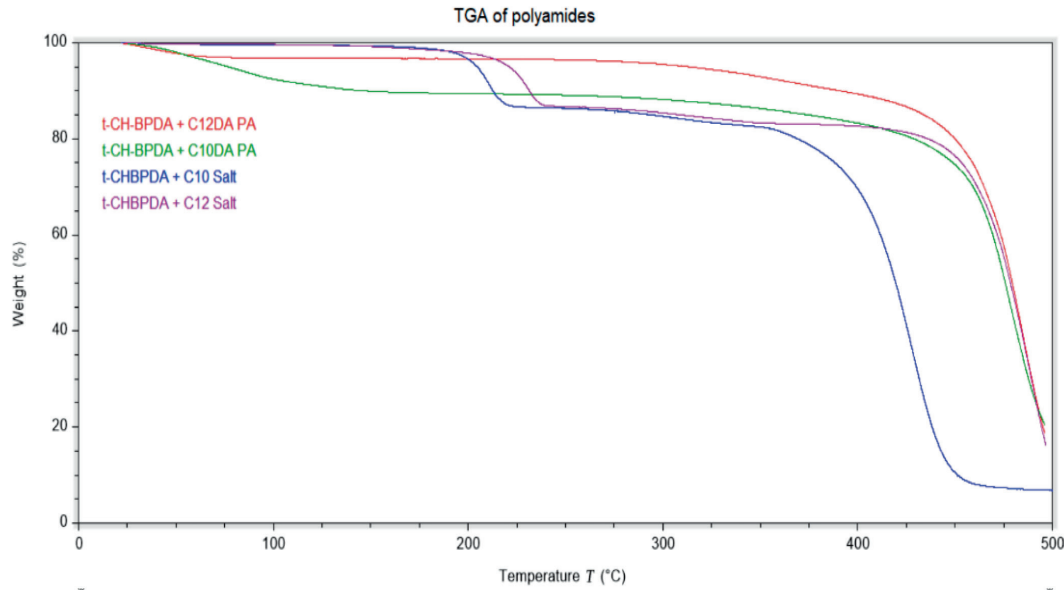

Figure S5 - 22. TGA analysis of organic salt of $t$-CH-BPDA with 1,10-decanediamine (blue), and the organic salt of $t$-CH-BPDA with 1,12-dodecanediamine (purple), and the corresponding polyamides (green, and red, respectively). 


\section{NMR analysis of BPDA-based polyesters}

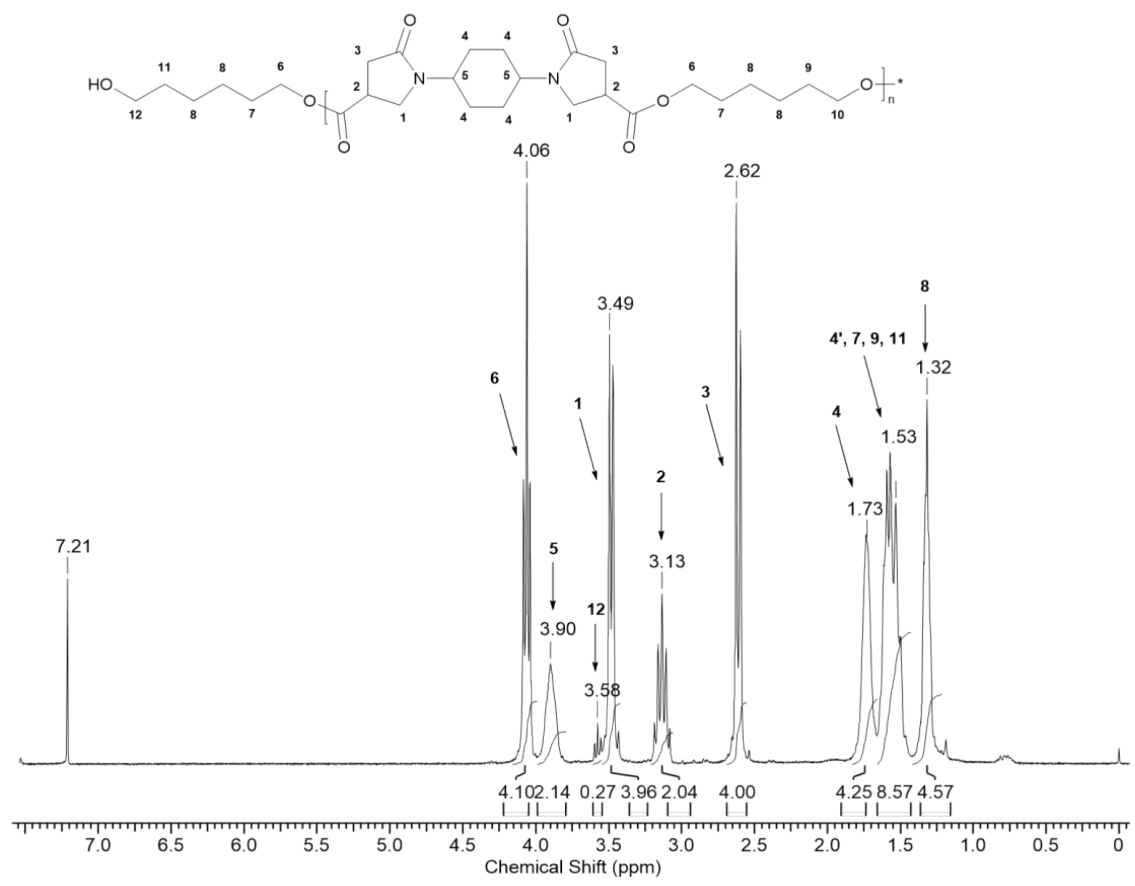

Figure $\mathrm{S} 5-23 .{ }^{1} \mathrm{H}$ NMR analysis of poly $\left([t-\mathrm{CH}-\mathrm{BPDA}]-\mathrm{C}_{6}\right)$.

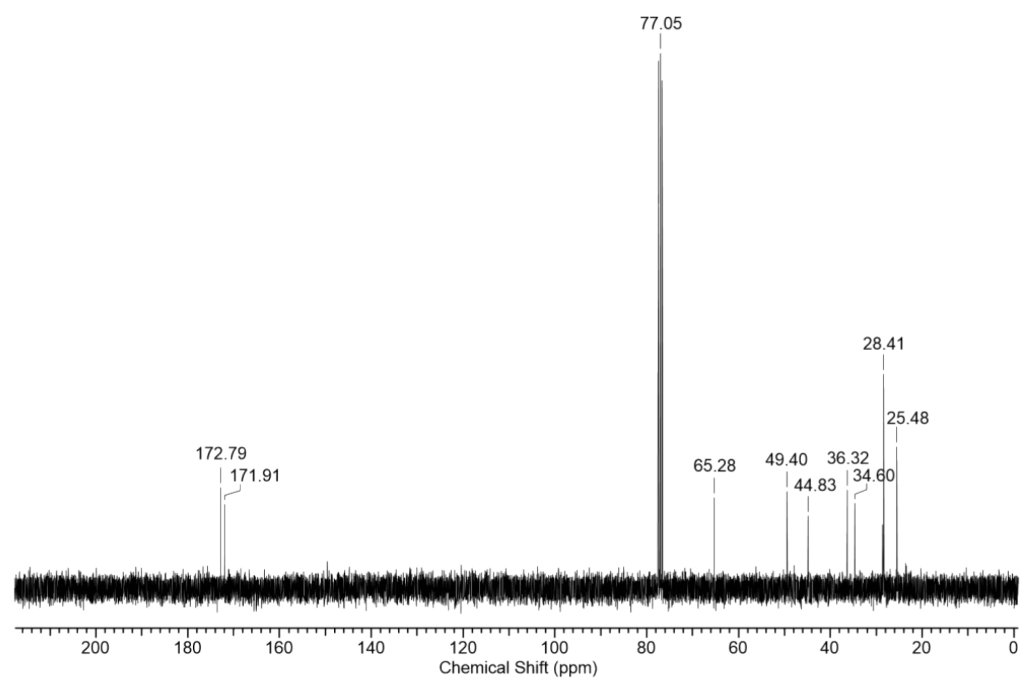

Figure S5 - 24. ${ }^{13} \mathrm{C}$ NMR analysis of poly([t-CH-BPDA $\left.]-\mathrm{C}_{6}\right)$. 


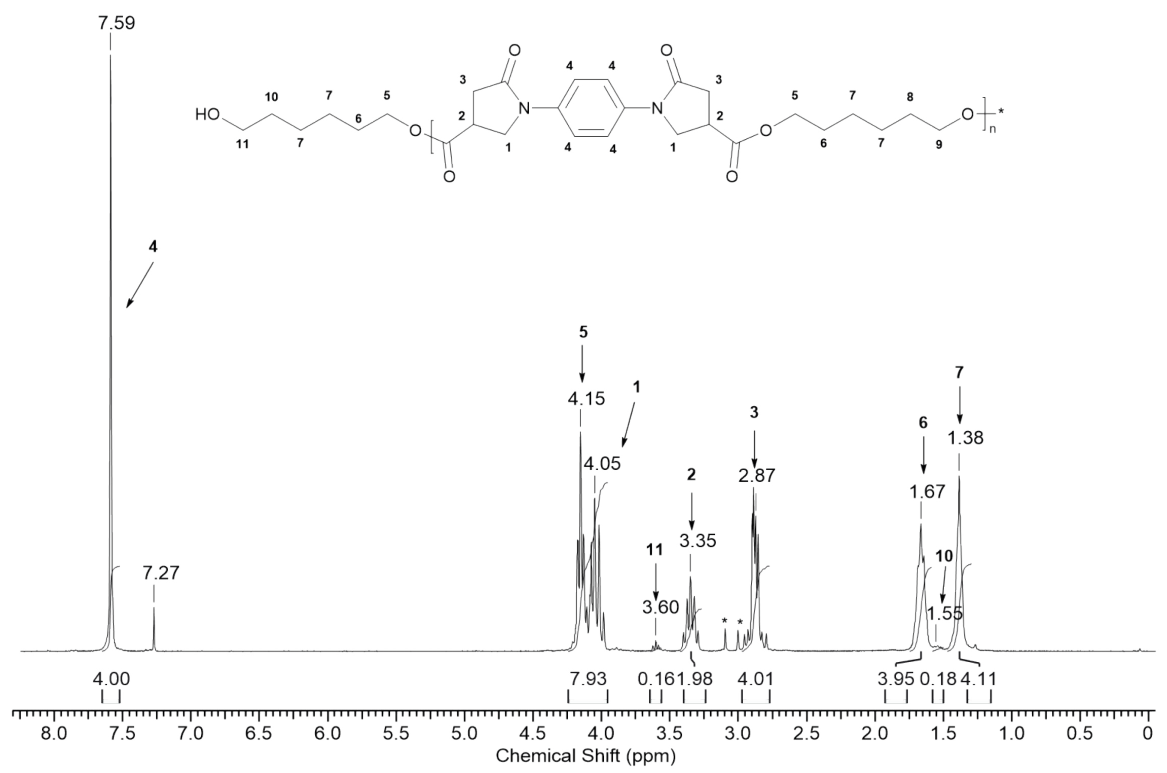

Figure S5 - 25. ${ }^{1} \mathrm{H}$ NMR analysis of poly $\left([p-\mathrm{Ph}-\mathrm{BPDA}]-\mathrm{C}_{6}\right)$, traces of DMF are denoted with an *.

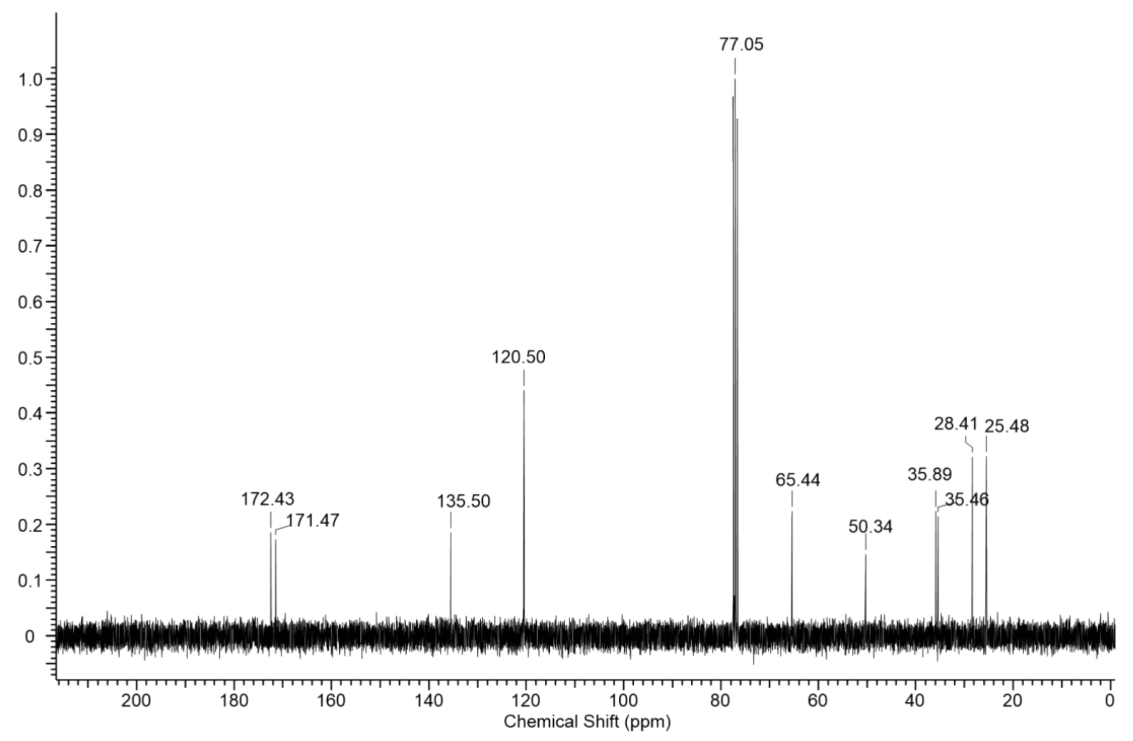

Figure S5 - 26. ${ }^{13} \mathrm{C}$ NMR analysis of poly $\left([p-\mathrm{Ph}-\mathrm{BPDA}]-\mathrm{C}_{6}\right)$. 


\section{IR analysis of BPDA-based polyesters}

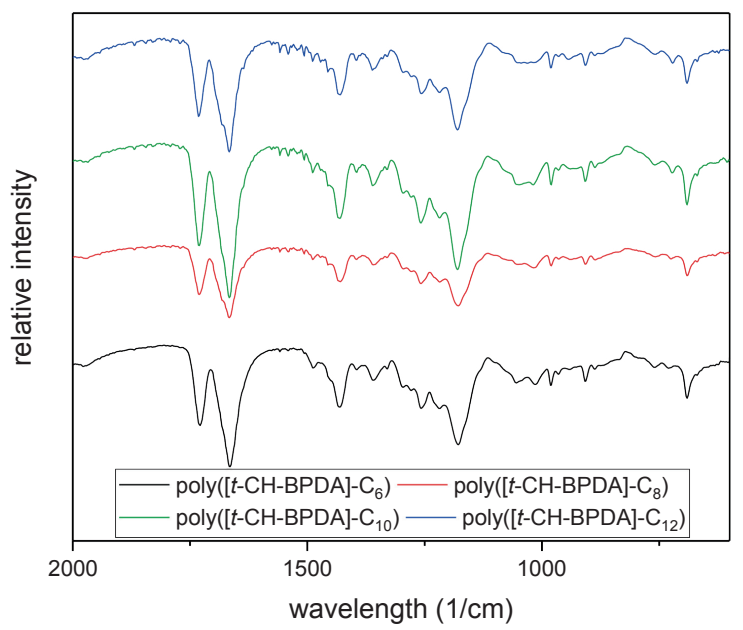

Figure S5 - 27. IR spectra for $t$-CH-BPDA-dm based polyesters, collected at room temperature. Poly $\left([t-\mathrm{CH}-\mathrm{BPDA}]-\mathrm{C}_{6}\right)$ (black), poly $\left([t-\mathrm{CH}-\mathrm{BPDA}]-\mathrm{C}_{8}\right)$ (red), Poly $\left(\left[t-\mathrm{CH}_{-}-\mathrm{BPDA}\right]-\mathrm{C}_{10}\right)$ (green), and poly $\left([t-\mathrm{CH}-\mathrm{BPDA}]-\mathrm{C}_{12}\right)$ (blue).

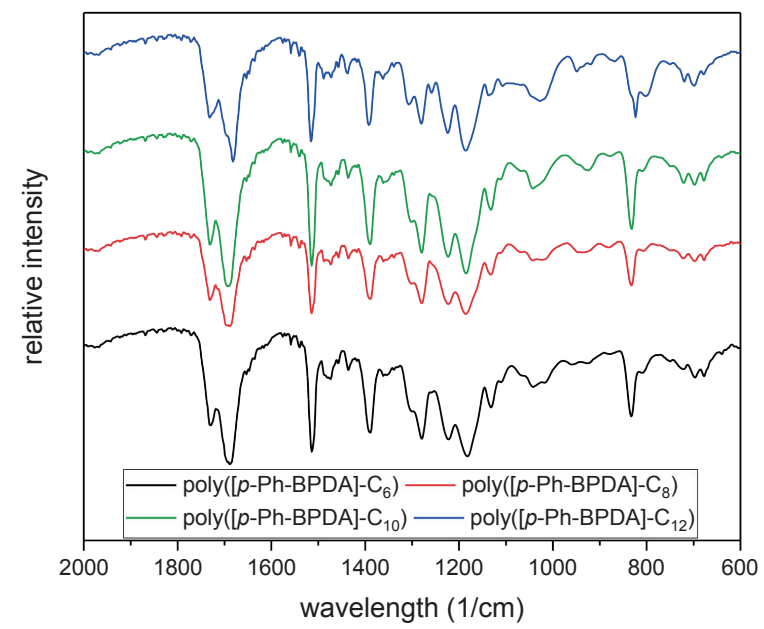

Figure S5 - 28. IR spectra for $p$-Ph-BPDA-dm based polyesters, collected at room temperature.

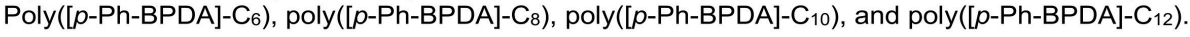




\section{DSC analysis of BPDA-based polyesters}

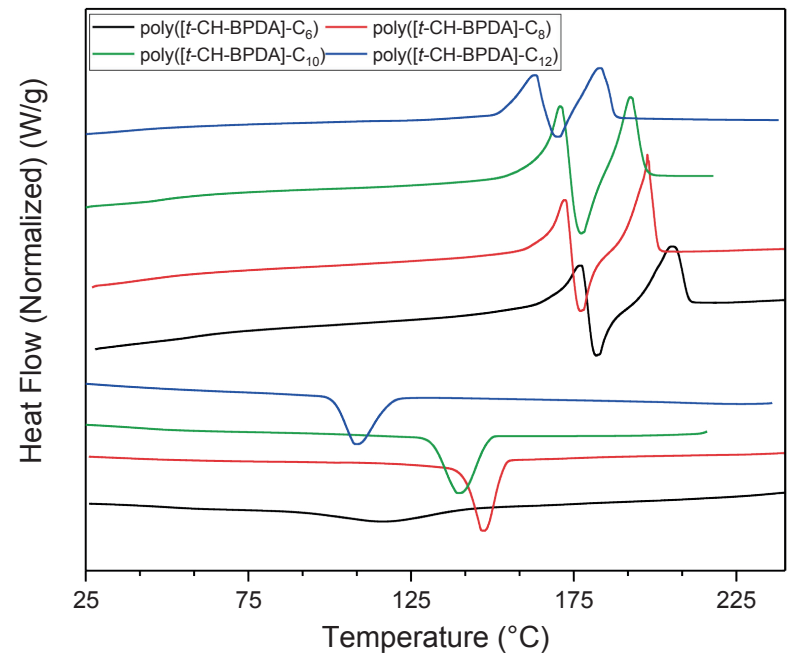

Figure S5 - 29. DSC thermograms of $t$-CH-BPDA-based polyesters. Bottom: first cooling of poly $\left([t-\mathrm{CH}-\mathrm{BPDA}]-\mathrm{C}_{12}\right)$ (blue, top), poly $\left([t-\mathrm{CH}-\mathrm{BPDA}]-\mathrm{C}_{10}\right)$ (green, $\left.{ }^{\text {nd }}\right)$, poly $\left([t-\mathrm{CH}-\mathrm{BPDA}]-\mathrm{C}_{8}\right)$ (red, $\left.3^{\text {rd }}\right)$, and poly $\left([t-\mathrm{CH}-\mathrm{BPDA}]-\mathrm{C}_{6}\right)$ (black, $\left.4^{\text {th }}\right)$. Top: second heating of poly $\left([t-\mathrm{CH}-\mathrm{BPDA}]-\mathrm{C}_{12}\right)$ (blue, top), poly $\left([t-\mathrm{CH}-B P D A]-\mathrm{C}_{10}\right)$ (green, $\left.{ }^{\text {nd }}\right)$, poly $\left([t-\mathrm{CH}-B P D A]-\mathrm{C}_{8}\right)\left(\right.$ red, $\left.3^{\text {rd }}\right)$, and poly $\left([t-\mathrm{CH}-\mathrm{BPDA}]-\mathrm{C}_{6}\right)$ (black, $\left.4^{\text {th }}\right)$. Heating and cooling rate of $10^{\circ} \mathrm{C} / \mathrm{min}$ was used.

\section{TGA water absorption test}

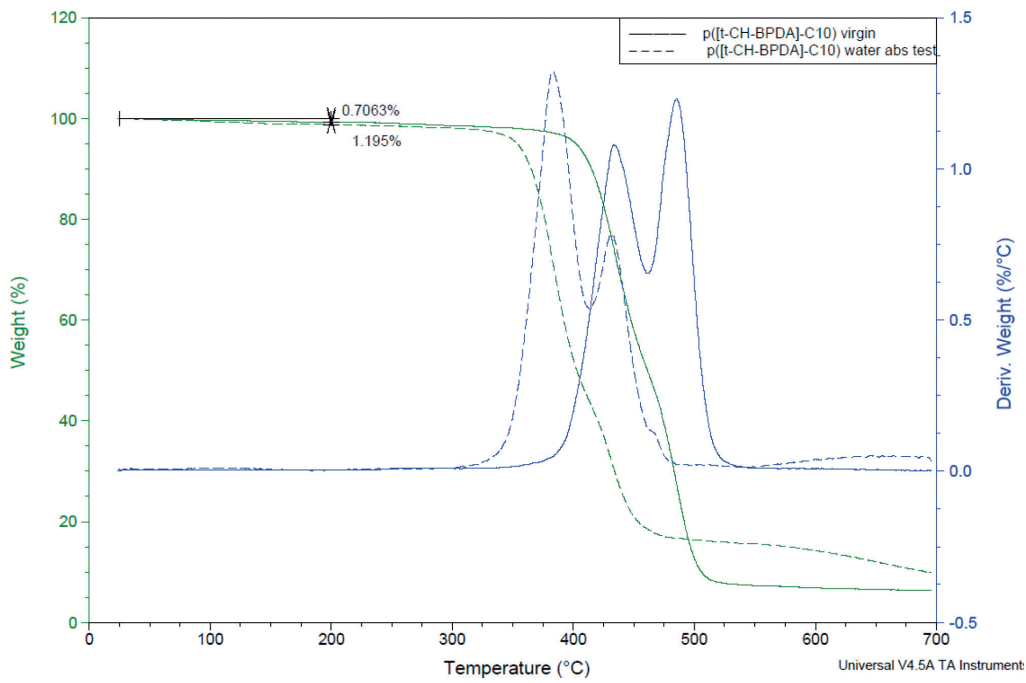

Figure S5 - 30. TGA analysis of water absorption of poly([t-CH-BPDA $\left.]-\mathrm{C}_{10}\right)$. Solid line: virgin material $(0.71 \mathrm{w} \%$ loss), dotted line: after 4 weeks in water ( $1.2 \mathrm{w} \%$ loss). 


\section{Chapter 6 On the mechanical performance of semi-crystalline bis(pyrrolidone) based polymers derived from itaconic acid.}

Manuscript in preparation

G.J. Noordzij, ${ }^{a, b}$ S. Rastogi, ${ }^{b}$ C.H.R.M. Wilsens ${ }^{b}$

${ }^{a}$ Chemelot InSciTe, Urmonderbaan 20F, NL-6167 RD Geleen, The Netherlands.

${ }^{b}$ Aachen-Maastricht Institute of Biobased Materials (AMIBM), Faculty of Science and Engineering, Maastricht University, Brightlands Chemelot Campus, 6167 RD Geleen, The Netherlands.

This chapter includes part of the bachelor internship research of Sanne van den Eijnde.

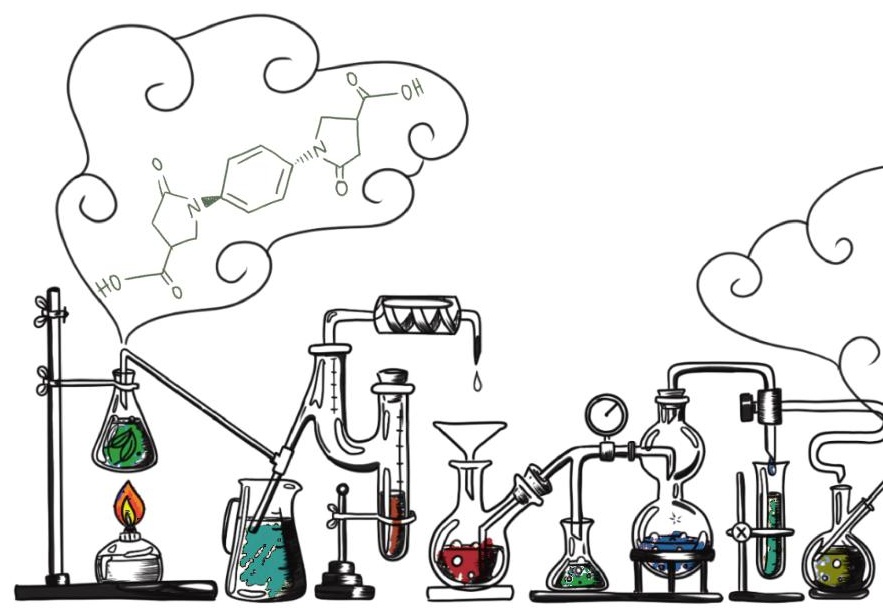




\section{Abstract}

The chapter addresses mechanical performance of the semi-crystalline bis-pyrrolidone-4carboxylic acids (BPDA) based polyesters described in chapter 5 of this thesis. Because of the brittle nature of the BPDA polyesters based on trans-1,4-cyclohexanediamine and 1,10dodecanediol (poly $\left.\left([t-\mathrm{CH}-\mathrm{BPDA}]-\mathrm{C}_{10}\right)\right)$, mechanical studies are found to be challenging. However, on copolymerization with 0.1-1.0\% EDTA crosslinker the mechanical performance of the polyester poly $\left([t-\mathrm{CH}-\mathrm{BPDA}]-\mathrm{C}_{10}\right)$ transforms from brittle to ductile. The transformation in the mechanical performance is related with the increase in the number of contact points between chains - chemical and physical entanglements. Though the addition of the cross-linker suppresses crystallization, it favors the formation of imperfect crystals suppressing the melting point by nearly $25^{\circ} \mathrm{C}$. The cross-linked amorphous films of poly ([t-CH-BPDA]-C $\left.{ }_{10}\right)$ having only $0.1 \% \mathrm{XL}$ could be cold-drawn up to $500 \%$ at room temperature. The pre-drawn amorphous samples, initially stretched up to $500 \%$, could be stretched further resulting into the uniaxial drawn films needing $94 \mathrm{MPa}$ stress to break, and having tensile modulus of $710 \mathrm{MPa}$. The increase in the energy to break is associated with the strain-induced crystallization perceived during the drawing process. On further annealing of the drawn films (at $60^{\circ} \mathrm{C}$ ) led to a higher degree of crystallization, causing jump in the tensile modulus from 710MPa to $3.1 \mathrm{GPa}$ and stress to break from $94 \mathrm{MPa}$ to $138 \mathrm{MPa}$. In contrast, the polymer having benzene moieties

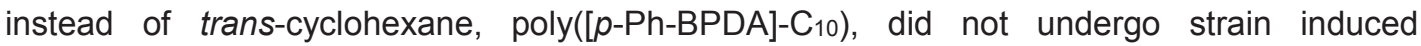
crystallization upon drawing. Nevertheless, drawn tapes of this polymer (up to $500 \%$ ) exhibit a tensile modulus of $3.65 \mathrm{GPa}$ and a strain at break of $362 \mathrm{MPa}$. The drawn film of poly $\left([p-P h-B P D A]-C_{10}\right)$ is found to regain its entropy on annealing above the glass transition temperature - leading to full shape-recovery within seconds. This shape-memory effect could be repeated 4 times without loss of mechanical performance.

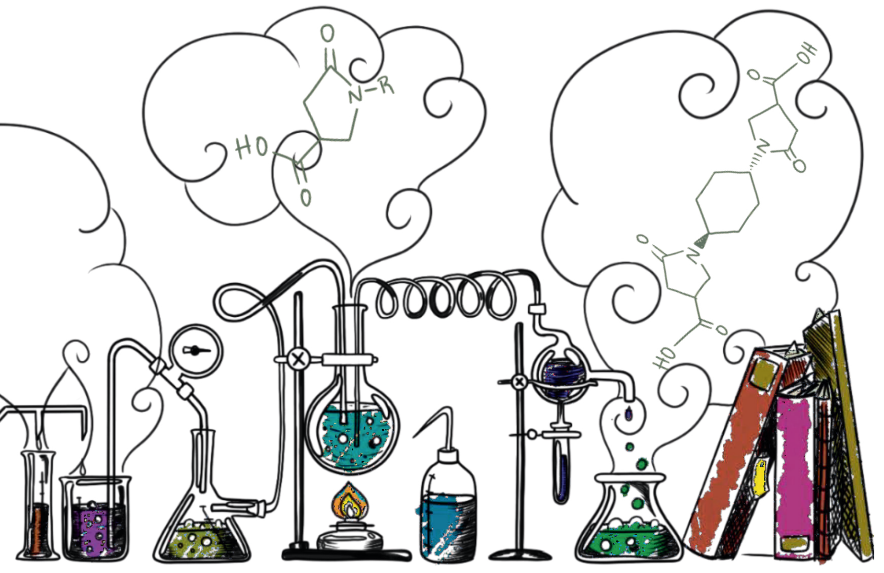




\section{Introduction}

In chapters 4 and 5 it is described how, through the aza-Michael addition-cyclization reaction, novel $N$ '-functionalized bis(pyrrolidone-2-carboxylic acid) (BPDA) have been synthesized and polymerized into various polyesters. Especially noteworthy are the polyesters based on trans-1,4-cyclohexanediamine $(t-\mathrm{CH})$ and para-phenylenediamine $(p-\mathrm{Ph})$, polymerized with long aliphatic diols such as 1,10-dodecanediol (Scheme 6-1), which are the first polymers of this class to show the capability of forming chain-folded crystals. ${ }^{1}$ Especially the polyesters based on $t$-CH-BPDA exhibits fast crystallization kinetics, and undergo a type of melt-reorganization-melt phase transition during heating between 160 and $210{ }^{\circ} \mathrm{C}$ (chapter 5). The polyester based on $p$-Ph-BPDA and 1,10dodecanediol (poly $\left(p-\right.$ Ph-BPDA-C$\left._{10}\right)$ ) also crystallizes and melts in standard DSC conditions, albeit with much slower rates, and without the observed phase-transition during the melt. Interestingly, both these polyesters also exhibit a very high stability in water, having minimal water-uptake and weight-loss after being submerged in water for prolonged times. The water-stability is unique since to date all reported BPDA-based polyesters suffered from high water uptake, which furthermore acts as a plasticizer, and causes hydrolytic degradation of the polymer films. ${ }^{2-6}$ The combined (fast) crystallization, high melting temperatures and high stability towards water makes these $t$-CH-BPDA and $p$-Ph-BPDA based polyesters highly promising materials for high performance applications.

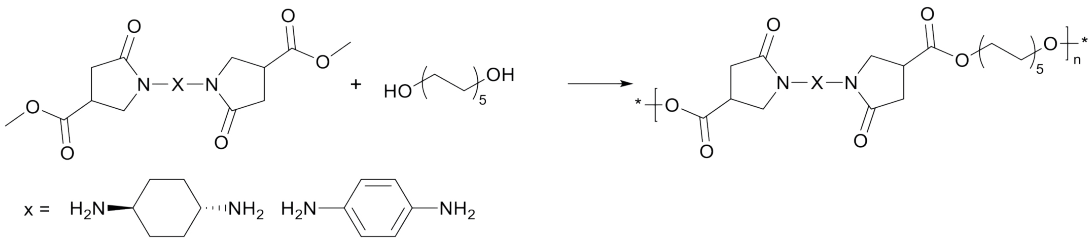

Scheme 6-1. Schematic overview of the polycondensation between either $t$-CH-BPDA-dm or $p$-Ph-BPDA-dm and $1,10-$ decanediol to obtain the BPDA-based polyesters used in this study.

For high-performance polymer materials it is essential that the polymers are tough, rather than brittle, and it is well known that crystallinity has a major effect on both the strength as the toughness of polymer materials. In order to assess the viability of these novel semi-crystalline BPDA-based polyesters towards high performance applications, this chapter describes preliminary mechanical properties, and the relation to crystallinity of the polyesters. To this end, polymer films of poly $\left(t-\mathrm{CH}-\mathrm{BPDA}-\mathrm{C}_{10}\right)$ and poly $(p-\mathrm{Ph}-\mathrm{BPDA}-$ $\left.C_{10}\right)$ have been prepared via vacuum-meltpressing, cut into dog bones and tested for mechanical performance via micro-tensile testing at different crystallization stages in the polymer films. 


\section{Materials and Methods}

\section{Materials}

Itaconic acid, para-phenylenediamine, 1,10-decanediol, ethylenediaminetetramethylacetate (EDTA), $\mathrm{Ti}(\mathrm{IV})$ isopropoxide and Irganox 1010 were obtained from Sigma Aldrich. Dimethyl itaconate and trans-1,4-cyclohexanediamine were obtained from TCl. Standard laboratory solvents were obtained from Biosolve. Toluene was distilled and stored over molsieves prior to use. Deuterated solvents were obtained from Buchem BV (Netherlands). The purchased compounds were used directly without further purification, unless otherwise specified.

\section{Synthesis methods}

Monomer synthesis was performed according to the protocols described in chapter 5 . The synthesis protocols for the dimethyl esters of $N, N^{\prime}$-trans-1,4-cyclohexane-bis(pyrrolidone-4-carboxylic acid), ( $t$-CH-BPDA-dm, Scheme 6-2, top), and $N, N^{\prime}$-trans-1,4-phenylene-bis(pyrrolidone-4-carboxylic acid), ( $p$-Ph-BPDA-dm, Scheme 6-2, bottom) are briefly recalled.
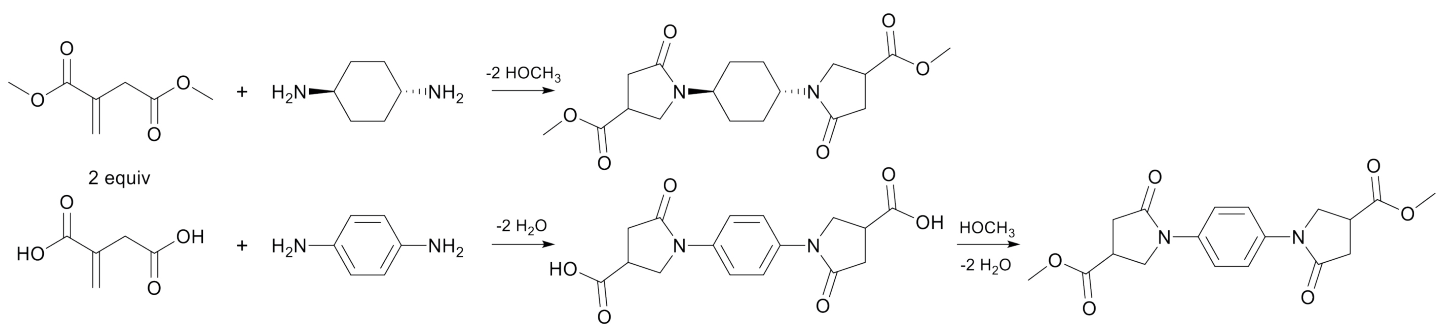

Scheme 6-2. Overview of the synthesis routes for the monomers $t$-CH-BPDA-dm (top) and $p$-Ph-BPDA-dm (bottom) used in this study.

\section{Synthesis of $t-C H-B P D A-d m$}

Trans-1,4-cyclohexanediamine $(25 \mathrm{~g}, 0.22 \mathrm{mmol})$ and dimethyl itaconate $(79.6 \mathrm{~g}, 0.5 \mathrm{~mol})$ were dissolved in $30 \mathrm{~mL}$ of methanol in a $250 \mathrm{~mL}$ round-bottom flask. The reaction mixture was stirred at reflux overnight, and after cooling the product was isolated after filtration as white crystals. Yield $90 \%(72.5 \mathrm{~g}) .{ }^{1} \mathrm{H}$ NMR $\left(\mathrm{CDCl}_{3}, 300 \mathrm{MHz}\right): \delta 3.97(\mathrm{~m}, 2.0 \mathrm{H}), 3.75(\mathrm{~s}, 6.01 \mathrm{H}), 3.54(\mathrm{~m}, 4.38 \mathrm{H}), 3.22(\mathrm{p}, 2.06 \mathrm{H}), 2.68(\mathrm{~m}, 4.29 \mathrm{H})$, $1.80(\mathrm{~m}, 4.22 \mathrm{H}), 1.59(\mathrm{~m}, 4.21 \mathrm{H}) .{ }^{13} \mathrm{C} \mathrm{NMR}\left(\mathrm{CDCl}_{3}, 300 \mathrm{MHz}\right): \delta$ 173.2, 172.0, 52.5, 49.4, 44.9, 36.2, 34.6, 28.4 .

\section{Synthesis of $p-P h-B P D A-d m$}

The synthesis of $p$-Ph-BPDA-dm was performed in a two steps. First, para-phenylenediamine (3.25 g, 30.1 $\mathrm{mmol}$ ) and itaconic acid $(7.83 \mathrm{~g}, 60.2 \mathrm{mmol})$ were loaded in a $100 \mathrm{~mL}$ round-bottom flask. Next, the reaction mixture was heated to $130{ }^{\circ} \mathrm{C}$ and stirred overnight. The product was obtained after recrystallization from water to yield $N, N^{\prime}$-trans-1,4-phenylene-bis(pyrrolidone-4-carboxylic acid), i.e. $p$-Ph-BPDA, as a white solid in $99 \%$ yield $(11.5 \mathrm{~g}) .{ }^{1} \mathrm{H}$ NMR $\left(\mathrm{CDCl}_{3}, 300 \mathrm{MHz}\right): \delta 7.55(\mathrm{~s}, 4.0 \mathrm{H}), 4.26(\mathrm{~m}, 4.04 \mathrm{H}), 3.60(\mathrm{p}, 2.09 \mathrm{H}), 3.20$

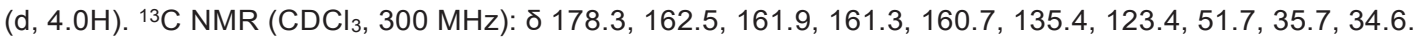

In the second reaction step, $5.1 \mathrm{~g}(15.3 \mathrm{mmol})$ of $p$-Ph-BPDA was suspended in $100 \mathrm{~mL}$ methanol and 2 $\mathrm{mL}$ of sulfuric acid in a $250 \mathrm{~mL}$ round-bottom flask. The methanol and generated water were removed in vacuo after refluxing for 72 hours. The obtained product was re-suspended in $100 \mathrm{~mL}$ methanol and again refluxed for 18 hours. After cooling the product was isolated in $81 \%$ yield as a grey solid after filtration $(4.45$ g). ${ }^{1} \mathrm{H} \mathrm{NMR}\left(\mathrm{CDCl}_{3}, 300 \mathrm{MHz}\right): \delta 7.54(\mathrm{~s}, 4.0 \mathrm{H}), 3.99(\mathrm{~m}, 4.15 \mathrm{H}), 3.72(\mathrm{~s}, 5.97 \mathrm{H}), 3.31(\mathrm{p}, 2.16 \mathrm{H}), 2.84(\mathrm{~m}$, 4.19H). 


\section{Polymer synthesis}

Melt-polycondensations with $p$-Ph-BPDA-dm or $t$-CH-BPDA-dm and 1,10-decanediol was achieved via a two-step melt polymerization: an initial esterification (oligomerization) step, followed by transesterification (polymerization) in the melt under reduced pressure ( $<0.01 \mathrm{mbar}$ ). Generally, the dimethyl ester and diol were loaded in a 1:1.05 molar ratio, including Irganox 1010 stabilizer in $0.05 \mathrm{mmol} \%$. For the cross-linked polymers based on $t$-CH-BPDA-dm, the desired ratio of EDTA was added $(0.10 .5,1.0$, or $2.5 \mathrm{~mol} \%)$ as replacement for the used dimethyl ester. The reaction flask with the reactants was purged 3 times with a vacuum $/ \mathrm{N}_{2}$ cycle before heating. For the oligomerization step the reaction mixture was gradually heated to $140{ }^{\circ} \mathrm{C}$ under a constant light $\mathrm{N}_{2}$-flow, after which the catalyst $(0.1 \mathrm{~mol} \%$ of $\mathrm{Ti}(\mathrm{IV})$ isopropoxide in toluene $)$ was added. The temperature was gradually increased to $180{ }^{\circ} \mathrm{C}$ and stirred 3 hours during which the condensate was distilled off. For the polymerization step the reaction mixture was heated to $210{ }^{\circ} \mathrm{C}$, and vacuum was applied for 4-6 hours to allow build-up of molecular weight. Lastly, the round bottom flask was placed under nitrogen atmosphere after which the polymers were removed from the hot reactor flask.

\section{Characterization methods}

Molecular weight $\left(M_{\mathrm{n}}, M_{\mathrm{w}}\right)$ and dispersity $(\Theta)$ of the polymers was determined from gel permeation chromatography (GPC). Experiments were performed on a Prominence-I LC-2030 equipped with a Shodex GPC KF-805L column. Analytical grade $\mathrm{CHCl}_{3}$ was used as mobile phase at $40{ }^{\circ} \mathrm{C}$, with a flowrate of 1 $\mathrm{mL} / \mathrm{min}$. Roughly $5 \mathrm{mg}$ of polymer was dissolved $1.5 \mathrm{~mL}$ of chloroform overnight under constant shaking, after which the samples were filtered over a $0.2 \mu \mathrm{m}$ PTFE syringe filter prior to injection. Thermal stability of compounds were performed via thermogravimetric analysis (TGA) using a TA Instruments Q500. Experiments were performed under a nitrogen atmosphere with a heating rate of $10{ }^{\circ} \mathrm{C} / \mathrm{min}$. Thermal transition temperatures of the polymers were analyzed via differential scanning calorimetry (DSC) using a TA Instruments DSC Q2000. Typically, two heating and cooling runs were performed at a rate of $10^{\circ} \mathrm{C} / \mathrm{min}$, the first heating was used to erase any thermal history in the samples. The glass-transition temperature $\left(T_{\mathrm{g}}\right)$, peak melt-temperature $\left(T_{\mathrm{m}}\right)$, peak crystallization temperature $\left(T_{\mathrm{c}}\right)$, and cold-crystallization temperature $\left(T_{\mathrm{cc}}\right)$ were obtained from the second heating and cooling run. DSC samples were prepared by loading 3-5 mg oven-dried samples in Tzero Hermetic Aluminum pans. Tensile tests were performed to assess the mechanical performance of the thermosets on small scale. To this end, a Linkam micro tensile stage, equipped with a constant force of $50 \mathrm{~N}$ load cell with accuracy of $0.01 \mathrm{~N}$ was used. Samples were cut into a flat dog bone type specimen with an effective gage dimensions of $13 \times 2 \times 1 \mathrm{~mm}$ (length $\times$ width $x$ thickness). The tensile tests were performed at a deformation rate of $100 \mathrm{~mm} / \mathrm{min}$. All the measurements were performed at room temperature. 2D-Wide angle $x$-ray diffraction (WAXD) analysis on the compression molded tensile bars was performed using a SAXSLAB Ganesha diffractometer using CuKa radiation $(\lambda=1.5406 \AA)$ and silver behenate $(d 001=58.380 \AA)$ as calibrant. Dynamic mechanical thermal analysis (DMTA) was performed on a Mettler Toledo DMA 1 Star System to identify the $T_{\mathrm{g}}$ of both the isotropic and oriented films. Temperature ramps were performed between 20 and $100{ }^{\circ} \mathrm{C}$ (heating rate of $5{ }^{\circ} \mathrm{C} / \mathrm{min}$ ) on films $2 \mathrm{~cm}$ in length at a frequency of $1 \mathrm{~Hz}$, an oscillatory stress of $0.1 \mathrm{~N}$ and a displacement of 10 micrometer were used for the experiments. 


\section{Results and Discussion}

Polymer synthesis and thermal characterization.

Preliminary studies on pressing films of poly $\left(t-\mathrm{CH}-\mathrm{BPDA}_{-} \mathrm{C}_{10}\right)$ indicated that the films were brittle, even with an average molecular weight $\left(M_{\mathrm{w}}\right)$ nearing $50 \mathrm{~kg} / \mathrm{mol}$ (Table 6-1). The cause for the observed brittle failure is likely to be a result of a too low number of entanglements per polymer chain, and thus a limited ability to delocalize the applied macroscopic stress along the molecular length scale. ${ }^{7}$ Therefore, in this work we introduce various concentrations of methylated EDTA cross-linker to A) increase the molecular weight of the polymer and $B$ ) to decrease the molecular weight between entanglements $\mathrm{Me}$. This way, EDTA is used as comonomer to increase the number of entanglements per polymer chain with the aim to facilitate a brittle to ductile transition in the mechanical performance of these materials. Copolymerizations with EDTA proved to be successful as the samples were partially (0.1 - $1.0 \mathrm{wt} \%$ EDTA) or fully (2.5 wt $\%$ EDTA) cross-linked. Note, as a result of this crosslinking process, the GPC data only reflects the soluble component of this material for the polymers containing $0.1-1.0 \mathrm{wt} \%$ EDTA (Table 6-1). Though, even with measuring only the soluble fractions, the effect of EDTA can clearly be seen by the sharp rise in $M_{\mathrm{w}}$ from $50 \mathrm{k} / \mathrm{mol}(0 \%)$ to $300 \mathrm{~kg} / \mathrm{mol}(1 \%)$. No GPC analysis was conducted on the polymer having $2.5 \mathrm{wt} \%$ EDTA as the cross-linked polymer could not be dissolved. The obtained polymers are named according to their composition, for example, the polymer containing $t$-CH-BPDA-dm, 1,10-decanediol, and $1.0 \%$ EDTA is abbreviated as poly $\left(t-\mathrm{CH}-\mathrm{BPDA}-\mathrm{C}_{10}\right)-1.0 \mathrm{XL}$. It is important to note that films poly $\left(p-P h-B P D A-C_{10}\right)$ were not brittle, and therefore did not require copolymerization with the cross-linker EDTA to increase the number of entanglements per polymer chain required for ductile macroscopic behavior.

Table 6-1. Overview of synthesized polymers based on 1,10-decanediol, their composition, numbers average $\left(M_{\mathrm{n}}\right)$ and weight average $(M \mathrm{w})$ molecular weight and dispersity $(\boxminus)$ from $\mathrm{CHCl}_{3}-\mathrm{GPC}$ results. ${ }^{\text {an }}$ Not measured due to insolubility.

\begin{tabular}{|c|c|c|c|c|c|}
\hline Polymer & Dimethyl ester & $\begin{array}{c}\text { EDTA } \\
\text { (mol\%) }\end{array}$ & $\begin{array}{c}M_{\mathrm{n}} \\
(\mathrm{kg} / \mathrm{mol})\end{array}$ & $\begin{array}{c}M_{\mathrm{w}} \\
(\mathrm{kg} / \mathrm{mol})\end{array}$ & $\boxplus(-)$ \\
\hline$p o l y\left(p-P h-B P D A-C_{10}\right)$ & p-Ph-BPDA-dm & 0 & 40.4 & 87.7 & 2.2 \\
\hline poly $\left(t-C H-B P D A-C_{10}\right)$ & $t$-CH-BPDA-dm & 0 & 14.4 & 49.6 & 3.5 \\
\hline poly $\left(t-C H-B P D A-C_{10}\right)-0.1 X L$ & $t-\mathrm{CH}-\mathrm{BPDA}-\mathrm{dm}$ & 0.1 & 22.8 & 74.6 & 3.3 \\
\hline poly $\left(t-C H-B P D A-C_{10}\right)-0.5 X L$ & $t-\mathrm{CH}-\mathrm{BPDA}-\mathrm{dm}$ & 0.5 & 17.7 & 63.5 & 3.6 \\
\hline poly $\left(t-C H-B P D A-C_{10}\right)-1.0 X L$ & $t-\mathrm{CH}-\mathrm{BPDA}-\mathrm{dm}$ & 1.0 & 22.2 & 303.4 & 13.7 \\
\hline poly $\left(t-C H-B P D A-C_{10}\right)-2.5 X L$ & $t-\mathrm{CH}-\mathrm{BPDA}-\mathrm{dm}$ & 2.5 & $-a$ & $-a$ & $-\mathrm{a}$ \\
\hline
\end{tabular}

As described in chapter 5 in this thesis, the BPDA-based polyesters, having $t$ - $\mathrm{CH}$ and $p$ $\mathrm{Ph}$ as the monomers, are the first BPDA-based polyesters to have semi-crystalline properties. Especially $t$-CH-BPDA based polyesters display interesting melting behavior as the polymer exhibits an endotherm upon heating, followed by an exotherm and a 
second endotherm. To recall, in chapter 5 we attributed the first endotherm to the melting of defected crystallites, which recrystallizes into more perfect crystals exhibiting an exotherm. The re-crystallized component melts on heating further, resulting into the second endotherm. Given this melting and recrystallization process occurring upon heating, we aimed to identify the effect of the presence of EDTA on the crystallization and melting behavior of poly $\left(t-\mathrm{CH}-B P D A-C_{10}\right)$. Note, as shown before in chapter 5 , poly $\left(p-P h-B P D A-C_{10}\right)$ is slow to crystallize under the chosen experimental conditions and exhibits very broad melting and crystallization transitions with low enthalpy (Table 6-2). Figure 6-1 displays the characteristic heating and cooling curves of the poly( $t-C H-B P D A-$ $\mathrm{C}_{10}$ ) series with various amount of the cross-linker EDTA taken at a heating and cooling rates of $10^{\circ} \mathrm{C} / \mathrm{min}$. As expected, the poly $\left(t-\mathrm{CH}-\mathrm{BPDA}_{-} \mathrm{C}_{10}\right)$ displays two endotherms: The first endothermic peak is observed at $170{ }^{\circ} \mathrm{C}$, which is followed by an exotherm attributed to crystallization and a second endotherm with a peak value at $196{ }^{\circ} \mathrm{C}$. The introduction of EDTA in the polymer results in an increase in the enthalpy of the first endothermic transition at $170{ }^{\circ} \mathrm{C}$ (Figure 6-1), while facilitating a decrease in the enthalpy in the second endothermic transition around $196{ }^{\circ} \mathrm{C}$. This data suggests that the presence of EDTA stimulates the formation of defected crystallites. Simultaneously, the total enthalpy of the melting-crystallization-melting transitions $\left(\Delta \mathrm{H}_{\mathrm{mcm}}\right.$, Table 6-2) decreases with increasing EDTA concentration (Table 6-2), suggesting that the presence of EDTA suppresses the overall ability of the material to crystallize.

Table 6-2. Overview of glass transition temperature $\left(T_{\mathrm{g}}\right)$, peak melting temperature $\left(T_{\mathrm{m}}\right)$, peak crystallization temperature $\left(T_{\mathrm{c}}\right)$, and enthalpy of the transitions $(\Delta \mathrm{H})$ observed during heating and cooling the polymers synthesized in this study in differential scanning calorimetry at a rate of $10{ }^{\circ} \mathrm{C} / \mathrm{min}$. a Only one melting transition observed for this polymer.

\begin{tabular}{|c|c|c|c|c|c|c|}
\hline Polymer & $T_{\mathrm{g}}\left({ }^{\circ} \mathrm{C}\right)$ & $T_{\mathrm{m}, 1}\left({ }^{\circ} \mathrm{C}\right)$ & $T_{\mathrm{m}, 2}\left({ }^{\circ} \mathrm{C}\right)$ & $\Delta H_{m c m}(J / g)$ & $T_{c}\left({ }^{\circ} \mathrm{C}\right)$ & $\Delta H_{c}(\mathrm{~J} / g)$ \\
\hline poly $\left(p-P h-B P D A-C_{10}\right)$ & 39.2 & 151.7 & - & 3.34 & 128.7 & 1.2 \\
\hline poly $\left(t-C H-B P D A-C_{10}\right)$ & 40.8 & 169.8 & 195.7 & 28.0 & 110.7 & 48.4 \\
\hline poly $\left(t-C H-B P D A-C_{10}\right)-0.1 X L$ & 42.9 & 172.2 & 196.3 & 24.5 & 121.6 & 46.1 \\
\hline poly $\left(t-C H-B P D A-C_{10}\right)-0.5 X L$ & 40.7 & 171.6 & 195.5 & 23.5 & 126.7 & 41.9 \\
\hline poly $\left(t-C H-B P D A-C_{10}\right)-1.0 X L$ & 40.9 & 169.4 & 194.4 & 21.7 & 118.7 & 37.9 \\
\hline poly $\left(t-C H-B P D A-C_{10}\right)-2.5 X L$ & 39.3 & 162.7 & 186.3 & 21.8 & 131.5 & 41.7 \\
\hline
\end{tabular}

Given that the EDTA acts as a cross-linker, it is likely to restrict the mobility of the polymer chains, thereby limiting the growth of the more 'perfect' crystals, thus favoring the growth of the crystals which melt around $170{ }^{\circ} \mathrm{C}$, and hampering the recrystallization towards the higher melting crystal phase. With respect to the crystallization during cooling from the melt, no clear trend is observed between the peak crystallization temperature and the EDTA content in the poly $\left(t-\mathrm{CH}-B P D A-C_{10}\right)$ copolymers. However, it is clear that the enthalpy of crystallization $\left(\Delta \mathrm{H}_{\mathrm{c}}\right.$, Table 6-2) decreases with increasing EDTA content, as expected from the decreasing enthalpy of the thermal transitions observed during heating. 

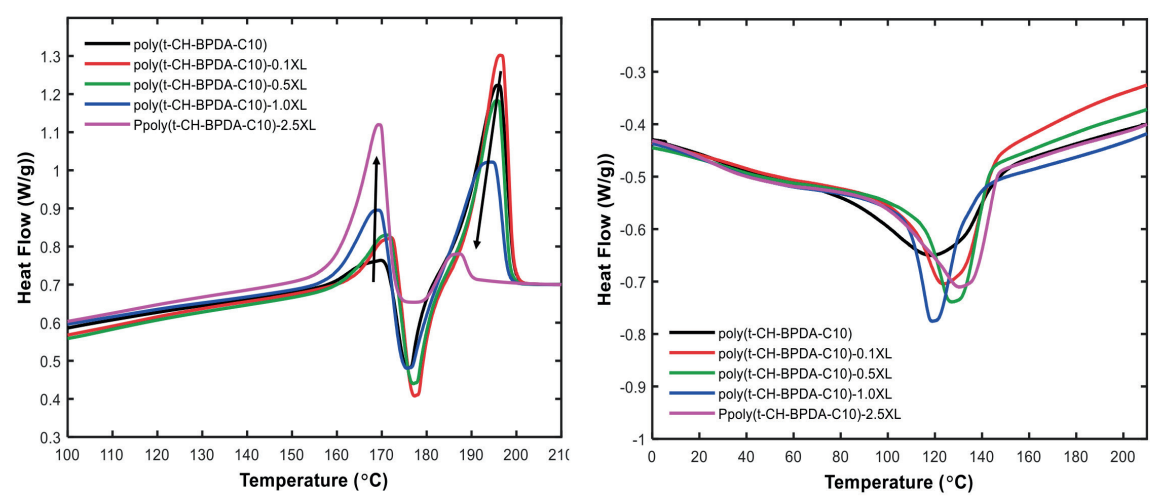

Figure 6-1. Left, characteristic melting behavior of the poly $\left(t-\mathrm{CH}-\mathrm{BPDA}_{-} \mathrm{C}_{10}\right)$ polymers having various amount of EDTA (second heating run). Right, crystallization observed while cooling from melt (first heating run). Note, prior to recording the first cooling run, the samples were heated to $220^{\circ} \mathrm{C}$ at a rate of $10^{\circ} \mathrm{C} / \mathrm{min}$ to erase all thermal history.

The polymer poly $\left(t-\mathrm{CH}-\mathrm{BPDA}_{-} \mathrm{C}_{10}\right)$ with $(0.1 \mathrm{wt} \%)$ and without EDTA were compression molded at $200{ }^{\circ} \mathrm{C}$ into thin films. The samples were prepared by rapid cooling from the melt to obtain amorphous polymer films. The quenched films were cut into dog bones, and were tested using a micro-tensile tester equipped with the load cells of $50 \mathrm{~N}$ at room temperature. It was observed that some samples cold-crystallized upon storage at room temperature, likely because the glass transition temperature is close to room temperature. Dog bone samples that were cold-crystallized, or purposely annealed, proved to be brittle, as can be seen by the low elongation and strain (Table 6-3). However, the mechanical performance of the poly( $\left.t-\mathrm{CH}-\mathrm{BPDA}-\mathrm{C}_{10}\right)-0.1 \mathrm{XL}$ changes drastically when subjecting it to tensile testing directly after processing, thereby avoiding cold-crystallization during storage.

Table 6-3. Overview of the obtained mechanical properties of the polymer films of poly $\left(t-C H-B P D A-C_{10}\right)-0.1 X L$ with the differentiation between normal dog bones pressed from the polymer film, or pre-drawn samples, and the differentiation between crystallized or non-crystallized samples.

\begin{tabular}{|c|c|c|c|c|c|c|}
\hline Polymer & pre-drawn & Crystallized & $\mathrm{E}(\mathrm{GPa})$ & $\sigma_{\text {break }}(\mathrm{MPa})$ & $\begin{array}{c}\text { Strain at } \\
\text { break (\%) }\end{array}$ & $\begin{array}{c}\text { number of } \\
\text { measurements }\end{array}$ \\
\hline poly $\left(t-C H-B P D A-C_{10}\right)$ & No & Yes & $0.55 \pm 0.08$ & $15.0 \pm 1.5$ & $5.3 \pm 0.34$ & 4 \\
\hline poly $\left(t-C H-B P D A-C_{10}\right)-0.1 X L$ & No & Yes & $0.74 \pm 0.02$ & $20.0 \pm 2.1$ & $3.6 \pm 1.8$ & 2 \\
\hline poly $\left(t-C H-B P D A-C_{10}\right)-0.1 X L$ & Yes & No & $0.71 \pm 0.07$ & $94.1 \pm 9.3$ & $58.1 \pm 20.5$ & 4 \\
\hline poly $\left(t-C H-B P D A-C_{10}\right)-0.1 X L$ & Yes & Yes & $3.1 \pm 0.1$ & $138 \pm 15$ & $32.9 \pm 4.5$ & 4 \\
\hline
\end{tabular}


In contrast to amorphous poly $\left(t-\mathrm{CH}_{-} \mathrm{BPDA}-\mathrm{C}_{10}\right)$, amorphous poly $\left(t-\mathrm{CH}-\mathrm{BPDA}-\mathrm{C}_{10}\right)-0.1 \mathrm{XL}$ allows for stretching up to the limit of the Linkam tensile stage ( $500 \%$ strain, Figure $6-2$, left). The samples undergoes deformation without showing any well-defined yield point, although it undergoes clear strain hardening at higher strains. The observation of strainhardening can be attributed to the cross-linked network in this polymer. The absence of a yield point is unexpected given that the glass transition temperature of this material is above room temperature $\left(40{ }^{\circ} \mathrm{C}\right)$. The absence of a yield point might originate from the absorption of a small amount of water (vapor), which is known to facilitate a plasticizing effect in amorphous BPDA based polymers. ${ }^{4}$ Given that the samples did not break during tensile testing, they were unloaded, cut into smaller pieces, and again subjected to mechanical analysis, which hereafter are called drawn tapes. Further mechanical tests on these drawn tapes were performed directly after tensile testing, and after annealing the samples for 1 hour at $60{ }^{\circ} \mathrm{C}$ (Figure 6-2, right). In general, we observe that drawn poly $\left(t-C H-B P D A-C_{10}\right)-0.1 X L$ tapes exhibit a tensile modulus $E$ of $710 \mathrm{MPa}$, and a yield point at 24.4 MPa and stretch well above $30 \%$ strain prior to failure. Furthermore, when annealing the drawn tapes to increase the crystallinity, the tensile modulus and stress at break increase to $3.1 \mathrm{GPa}$ and $138 \mathrm{MPa}$, respectively. Similar results were obtained for poly $\left(t-C H-B P D A-C_{10}\right)-0.5 X L$ and poly $\left(t-C H-B P D A-C_{10}\right)-1.0 X L$, which could both be stretched directly after compression molding. However, as no notable differences were observed in mechanical performance with increasing EDTA concentration, only the results from the poly $\left(t-\mathrm{CH}-\mathrm{BPDA}-\mathrm{C}_{10}\right)-0.1 \mathrm{XL}$ samples are discussed in detail.
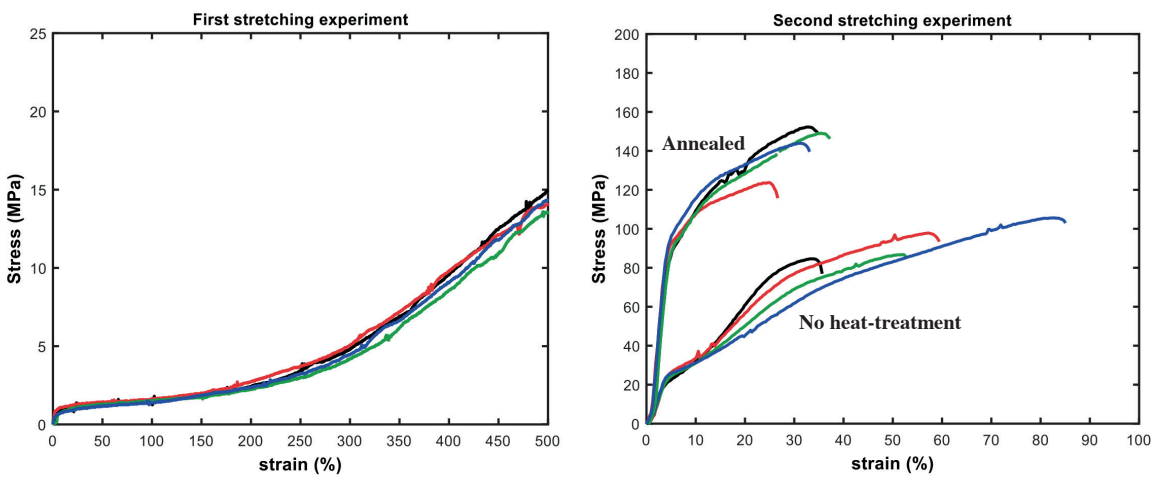

Figure 6-2. Characteristic stress-strain curves for poly $\left(t-\mathrm{CH}-B P D A-\mathrm{C}_{10}\right)-0.1 \mathrm{XL}$ in the amorphous state obtained directly after processing (left) and in a second stretching experiment of the drawn tapes before and after an annealing step at $60^{\circ} \mathrm{C}$ for 1 hour (right).

As is shown in Figure 6-3, the sample directly after compression molding (Figure 6-3A) is amorphous in nature, indicated by the presence of a broad isotropic halo and the absence of any diffraction peaks. Note, these samples display a second isotropic scattering signal close to the beam-stop (at $2 \theta=4, d=2.2 \mathrm{~nm}$ ), which corresponds to the length of the repeat unit of the poly $\left(t-\mathrm{CH}-B\right.$ PDA- $\left.\mathrm{C}_{10}\right)$ polymer (calculated estimation via ChemDraw 3D of $2.4 \mathrm{~nm}$ ). As can be deduced from Figure 6-3B, annealing of the amorphous dog bones lead to the formation of isotropic crystallites. Note: similar spectra were obtained for dog bones stored over time at room temperature. 2D-WAXD analysis 
on the drawn poly $\left(t-\mathrm{CH}-\mathrm{BPDA}_{10} \mathrm{C}_{10}-0.1 \mathrm{XL}\right.$ tapes (Figure 6-3C) (corresponding to the tensile experiment shown in Figure 6-2, left), suggests the presence of an anisotropic crystalline fraction which is apparent form the converging diffraction peaks towards the equator. Furthermore, the scattering signals originating from the electron density fluctuations between the $t$-CH-BPDA and the $\mathrm{C}_{10}$ spacer (hence, the polymers repeat unit), orient towards the meridian (drawing direction) and even display the presence of a second order diffraction signal (highlighted by the white arrow in Figure 6-3C). This strongly suggests that strain-induced crystallization occurs when stretching the poly $\left(t-\mathrm{CH}-\mathrm{BPDA}-\mathrm{C}_{10}\right)-0.1 \mathrm{XL}$ polymer, already at room temperature. Lastly, annealing of the drawn poly( $\left.t-\mathrm{CH}-\mathrm{BPDA}_{-} \mathrm{C}_{10}\right)-0.1 \mathrm{XL}$ samples results in a further increase in the crystalline content, detected by the further development of the diffraction signals (Figure 6-3D).
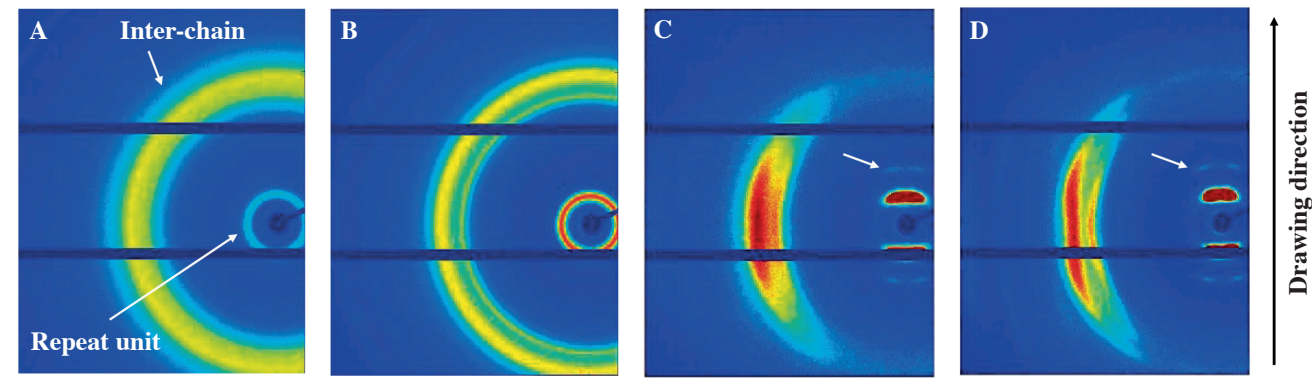

Figure 6-3. 2D-Wide angle $x$-ray diffraction patterns obtained for poly(t-CH-BPDA-C 10$)-0.1 \mathrm{XL}$ after $A)$ compression molding, B) compression molding and annealing, C) drawing, and D) drawing and annealing.

The origin of the enhancement in mechanical performance in the drawn poly $(t-C H-B P D A-$ $\mathrm{C}_{10)}-0.1 \mathrm{XL}$ samples is therefore likely related to the occurrence of strain induced crystallization. Such strain-induced crystallization of the amorphous poly $\left(t-C H-B P D A-C_{10}\right)-0.1 X L$ explains the sudden appearance of a yield point in the stretched samples and the increase in the tensile modulus. The modulus was further increased from $710 \mathrm{MPa}$ to 3.1GPa by annealing of the drawn tapes (Table 6-3), stimulating the growth of oriented crystals (Figure 6-3D), which indicates that indeed the strain-induced crystallization leads to desirable ductile properties, rather than brittle failure from isotopic crystallized samples. Overall, these findings demonstrate that the copolymerization with already low amounts of EDTA can effectively be used to increase the molecular weight of poly $\left(t-\mathrm{CH}-\mathrm{BPDA}_{-} \mathrm{C}_{10}\right)$ sufficiently to allow for drawing in the amorphous phase, and yield materials with promising mechanical performance. Though only for (crystallized) samples in the drawn state because the isotropically crystallized samples remain brittle. 


\section{Mechanical performance of poly $\left(p-P h-B P D A-C_{10}\right)$}

To recall, $\operatorname{poly}\left(p-\mathrm{Ph}-\mathrm{BPDA}-\mathrm{C}_{10}\right)$ having $M_{\mathrm{w}}$ of 87.7 proved sufficient for direct compression molding and tensile testing without being limited by the brittleness reported for poly $\left(t-\mathrm{CH}-\mathrm{BPDA}-\mathrm{C}_{10}\right)$. For this reason, no attempts were made to crosslink the poly $(p-$ $\left.\mathrm{Ph}-\mathrm{BPDA}-\mathrm{C}_{10}\right)$. The material was subjected to the same processing steps and mechanical analysis as performed for the poly $\left(t-\mathrm{CH}-\mathrm{BPDA}-\mathrm{C}_{10}\right)-0.1 \mathrm{XL}$ sample (Figure $6-4)$. Interestingly, despite the comparable $T_{\mathrm{g}}$ between poly $\left(t-\mathrm{CH}-\mathrm{BPDA}-\mathrm{C}_{10}\right)$ and poly $(p-$ Ph-BPDA- $\left.\mathrm{C}_{10}\right)$, poly $\left(p-\mathrm{Ph}_{-} \mathrm{BPDA}-\mathrm{C}_{10}\right)$ does display a yield point at $23.7 \pm 1.6 \mathrm{MPa}$ and a tensile modulus $E=1090 \pm 88 \mathrm{MPa}$. During the uniaxial deformation the sample is found to show necking and deformation by more than $400 \%$. It is apparent from the WAXD (Figure 6-5A), that prior to the uniaxial deformation the sample is found to be entirely amorphous, which is shown by the diffuse scattering signal in x-ray diffraction experiments. In combination to the isotropic halo attributed to the interchain distance, an isotropic halo close to the beamstop is also observed (at $5^{\circ} 2 \theta, 1.77 \mathrm{~nm}$ ), which is slightly shorter than the length of the repeat unit of poly $\left(t-\mathrm{CH}-B P D A-C_{10}\right)$ polymer (calculated estimation via ChemDraw 3D of $2.4 \mathrm{~nm}$ ).
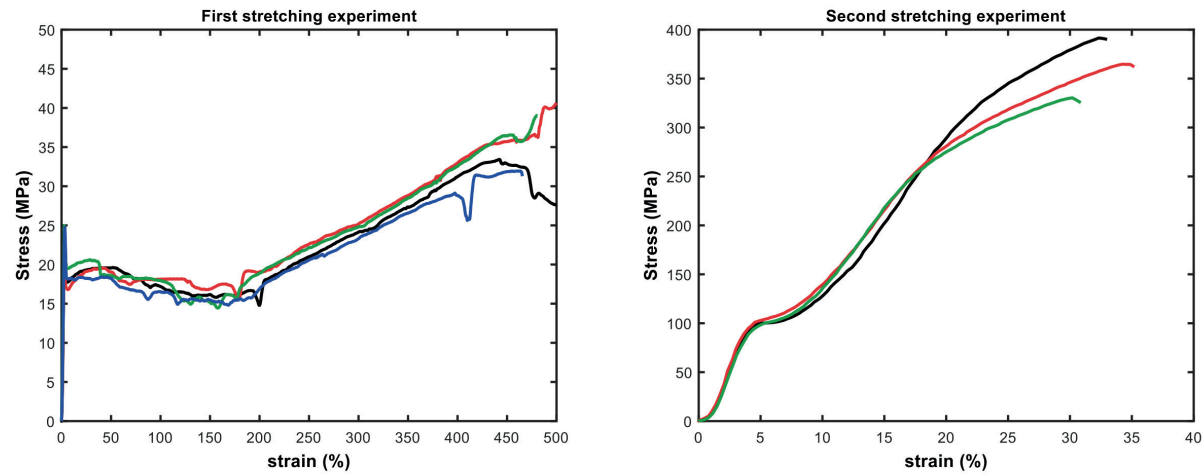

Figure 6-4. Characteristic stress-strain curves for poly( $\left.p-\mathrm{Ph}-\mathrm{BPDA}-\mathrm{C}_{10}\right)$ obtained directly after processing (left) and in a second stretching experiment of the drawn tapes (right).
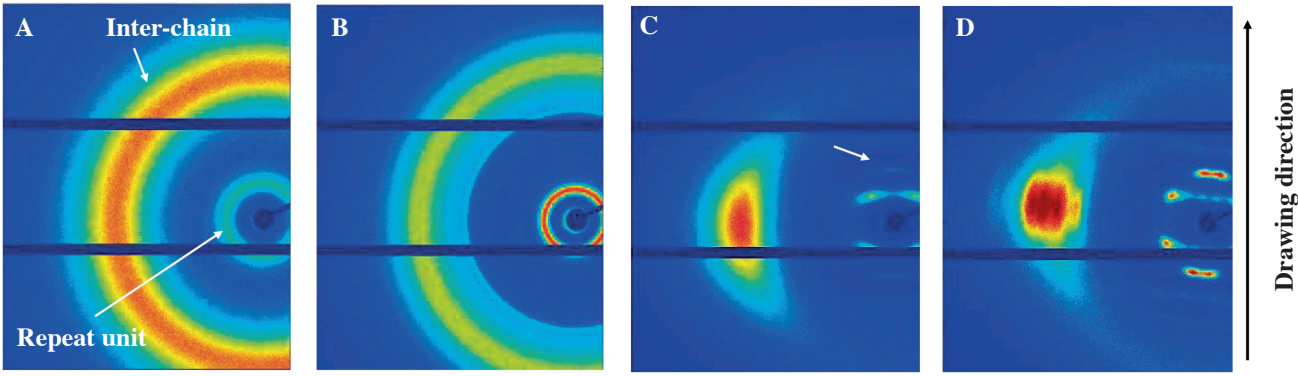

Figure 6-5. 2D-Wide angle x-ray diffraction patterns obtained from poly $\left(p-P h-B P D A-C_{10}\right)$ after $\left.A\right)$ compression molding, B) compression molding and annealing, C) drawn tapes of the amorphous compression molded sample at room temperature, and D) annealing of the drawn tape. 
Similar to poly $\left(t-\mathrm{CH}-\mathrm{BPDA}-\mathrm{C}_{10}\right)-0.1 \% \mathrm{XL}$, poly $\left(p-\mathrm{Ph}-\mathrm{BPDA}-\mathrm{C}_{10}\right)$ displays ductile deformation and readily undergoes strain-hardening during tensile deformation at room temperature (Figure 6-4, left). However no strain-induced crystallization is observed, indicated by the absence of diffraction peaks in the scattering signal corresponding to the inter-chain distance of the polymer chains (Figure 6-5C). Nonetheless, there exists a well-defined short-range order, as can be observed by the orientation of the scattering signal close to the beamstop, in combination with an higher order diffraction signal indicated by the white arrow in Figure $6-6 \mathrm{C}$. Similar to poly $\left(t-\mathrm{CH}-\mathrm{BPDA}-\mathrm{C}_{10}\right)-0.1 \mathrm{XL}$, the drawn amorphous samples were cut (drawn tapes), and subjected to a second drawing on the tensile tester (Figure 6-4, right). These drawn tapes have a yield tensile modulus of $\mathrm{E}=3.65 \pm 0.17 \mathrm{GPa}$ and a yield point at $100.5 \pm 0.5 \mathrm{MPa}$. More interestingly, these tapes can be stretched over $30 \%$ strain and fail at $362 \pm 30 \mathrm{MPa}$.

Though the drawn tapes are still amorphous after stretching (Figure 6-5C), the mechanical performance of these oriented poly $\left(p-\mathrm{Ph}-\mathrm{BPDA}-\mathrm{C}_{10}\right)$ samples is superior to the drawn poly $\left(t-\mathrm{CH}-\mathrm{BPDA}-\mathrm{C}_{10}\right)-0.1 \mathrm{XL}$ samples, in particular the stress at break. Note, crystallization of poly $\left(p-P h-B P D A-C_{10}\right)$ samples can be enforced by subjecting the samples to an annealing step at temperatures above the glass transition temperature (60 ${ }^{\circ} \mathrm{C}$ ), as can be observed for the polymer film (Figure 6-5B) and drawn tape (Figure 6-5D). However, annealing experiments of the stretched tapes proved challenging as they readily contracted back into their original shape, a feature well known to occur in shapememory materials.

The position of the integrated diffraction peak (Figure 6-6), close to the beam-stop, shows dependence on the mechanical and thermal treatment of the polymer films. The amorphous compression molded samples display a diffraction signal at $5^{\circ} 2 \varnothing$, corresponding to the d-spacing of $1.77 \mathrm{~nm}$. Interestingly, this diffraction signal shifts to $4.52^{\circ} 2 \varnothing$ after drawing, corresponding to a length of $1.96 \mathrm{~nm}$. The increase in periodic length (from 1.77 to $1.96 \mathrm{~nm}$ ) likely corresponds to the stretching of the polymer backbone. Annealing a drawn tape leads to some stress relaxation, however also gives rise to a sharp diffraction signal at $7.052 \varnothing$, corresponding to a length of $1.25 \mathrm{~nm}$. Given

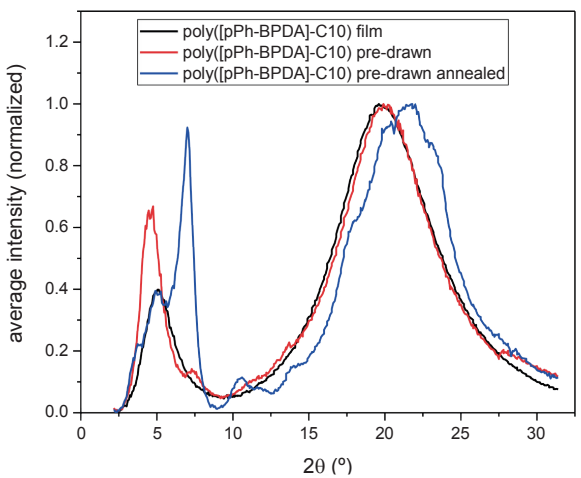

Figure 6-6. Integration of the WAXD diffraction signals of poly $\left(p-P h-B P D A-C_{10}\right)$ : amorphous film (black), pre-drawn (red) and pre-drawn annealed (blue). 
that the periodicity at a length of $1.25 \mathrm{~nm}$ is roughly half of the repeat unit in the extended chain configuration, we consider it likely that this diffraction signal arises from the periodic alternation between the BPDA and 1,10-decanediol subunits.

\section{Preliminary study on shape-memory behavior of poly $\left(p-P h-B P D A-C_{10}\right)$}

As briefly mentioned in the previous section, the pre-drawn poly $\left(p-P h-B P D A-C_{10}\right)$ samples readily contract back into their original dog-bone shape when heated to temperatures beyond their $T_{\mathrm{g}}$ (Figure 6-7). Considering there are no cross-links or semicrystalline domains in the pre-drawn sample, the shape memory behavior is likely merely a stress-relaxation process, similar to e.g. shrink-foils. During cold-drawing at room temperature the entangled network becomes partially aligned leading to an increase in $T_{\mathrm{g}}$, inherently also leading to a reduced entropy. The $T_{\mathrm{g}}$ increases from $39{ }^{\circ} \mathrm{C}$ (DSC) or $36{ }^{\circ} \mathrm{C}$ (DMTA, based on the peak value in E"), to $47^{\circ} \mathrm{C}$ (DSC) and $53^{\circ} \mathrm{C}$ (DMTA) during cold-drawing, as is shown in Figure 6-8. Due to the higher $T_{\mathrm{g}}$ the now oriented entangled network is kinetically trapped in this drawn state. Upon heating to above the $T_{\mathrm{g}}$ the network relaxes back to a random disordered state due to a gain in entropy, resulting in the observed shape-memory effect.
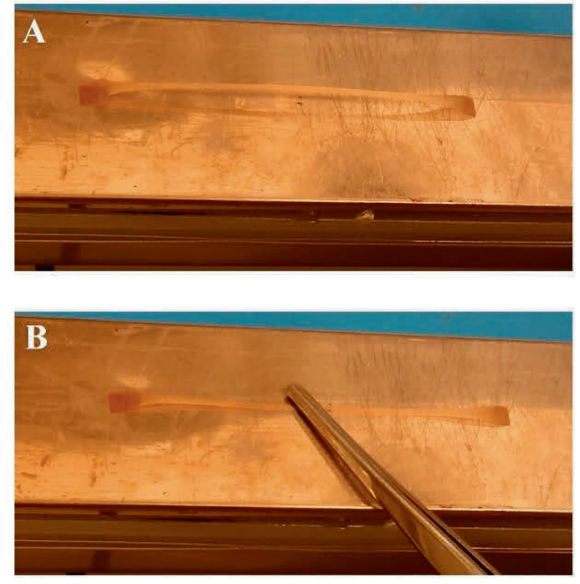
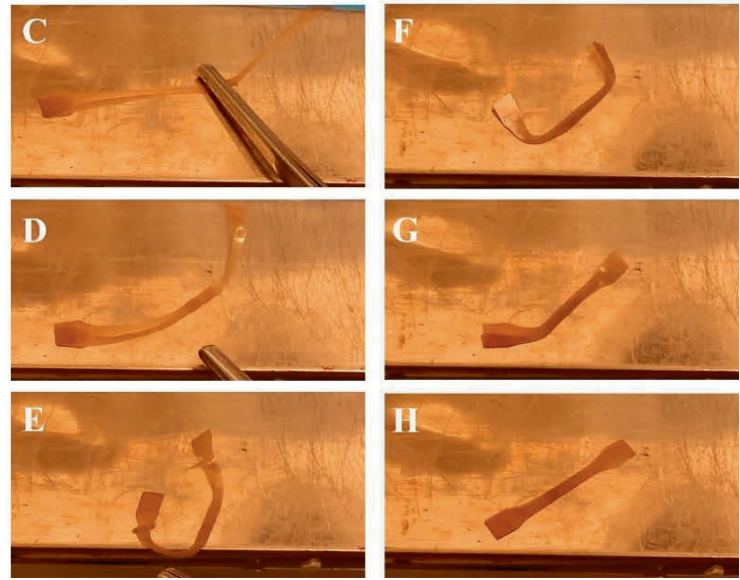

Figure 6-7. Images depicting the characteristic shape memory behavior of the oriented poly $\left(p-\operatorname{Ph}-\mathrm{BPDA}-\mathrm{C}_{10}\right)$ tape upon exposure to a Kofler bench with temperature above the glass transition temperature $\left(80-120^{\circ} \mathrm{C}\right)$. The relaxation proceeds within a few seconds following the order of the images $\mathrm{A}-\mathrm{H}$. Tweezers are used to bring the film in contact with the heated surface, after which the relaxation process proceeds. Note, the two sides of the samples have not been oriented as these were clamped in the Linkam tensile stage during the mechanical analysis.

Furthermore, from Figure 6-8 left it is apparent that a large overshoot in heat flow occurs when the of pre-drawn samples is heated beyond the $T_{\mathrm{g}}$. The overshoot in the heat flow is likely from the aforementioned stress relaxation and, hence, the contraction of the tape. As expected, the storage modulus of the oriented poly $\left(p-P h-B P D A-C_{10}\right)$ tape is significantly higher than that of the isotropic film, undoubtedly resulting from the polymer chains oriented along the drawing direction of the tape (and along the direction where 
the oscillatory stress is applied). However, upon heating beyond the $T_{\mathrm{g}}$ the storage does not decrease to the value of the isotropic film, instead it remains three orders of magnitude larger, suggesting that the sample still maintains its oriented structure. It is likely that the sample is unable to relax as it is fixed at both ends by the DMTA clamps. Irrespective of the origin of this shape-memory behavior, the orientation-relaxation process could be repeated up to four times prior to failure of the sample (Figure 6-9). These preliminary findings highlight a promising property of the novel poly $(p-\mathrm{Ph}-\mathrm{BPDA}$ $\left.\mathrm{C}_{10}\right)$ polymer.
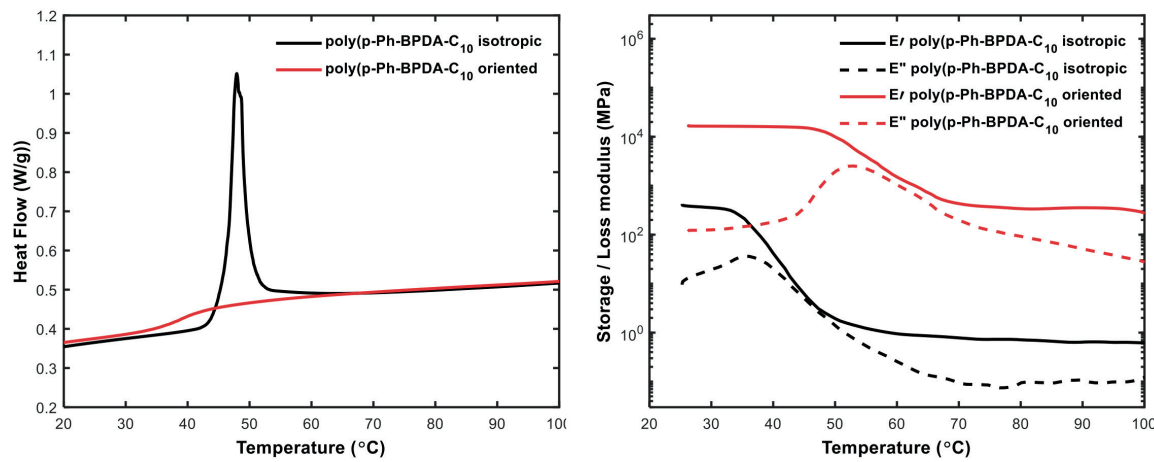

Figure 6-8. Left, heating run depicting the glass transition in the isotropic amorphous poly $(p-P h-B P D A-$ $C_{10}$ ) sample and in the oriented tape, obtained during heating at a rate of $10^{\circ} \mathrm{C} / \mathrm{min}$. Right, Storage ( $\left.E^{\prime}\right)$ and Loss (E") moduli of the same samples during a temperature ramp, as observed in DMTA analysis while heating at a rate of $5^{\circ} \mathrm{C} / \mathrm{min}$.

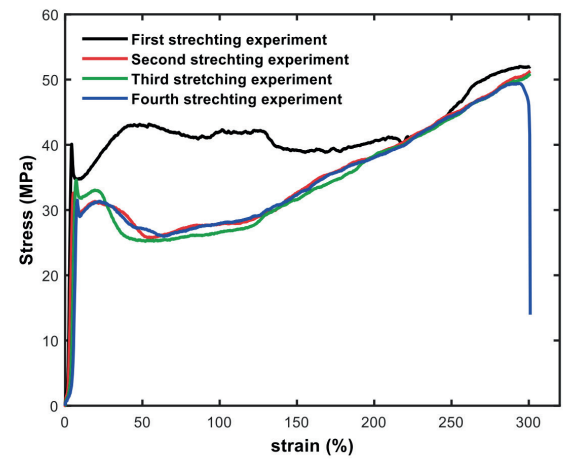

Figure 6-9. Stress vs strain plots of an isotropic amorphous poly $\left(p-\mathrm{Ph}-\mathrm{BPDA}_{\mathrm{C}} \mathrm{C}_{10}\right)$ film, which was stretched to $300 \%$ strain, taken out of the tensile tester, allowed to relax at temperatures above $T_{\mathrm{g}}$ and placed back in the tensile tester. This process could be repeated four times before failure occurred during stretching. 


\section{Conclusions}

In this work we report on the thermal behavior and mechanical performance of poly $(t-\mathrm{CH}-$ BPDA- $\left.\mathrm{C}_{10}\right)$ copolymers with various amount of EDTA as comonomer and of poly $(p-\mathrm{Ph}-$ BPDA-C 10 ). Both polymers, given that they have sufficiently high molecular weight and or contain cross-links, prove ductile during tensile testing at room temperature, although only when residing in the amorphous phase. Strain-induced crystallization occurs during tensile testing of the amorphous poly ( $p$-Ph-BPDA- $\left.\mathrm{C}_{10}\right)-0.1 \mathrm{XL}$, effectively enhancing the mechanical performance of the drawn samples (tapes). Annealing of these tapes increases crystallinity further and thereby yielding tapes with a tensile modulus of 3.1 $\mathrm{GPa}$ and a stress at break of $138 \mathrm{MPa}$. The poly $\left(p\right.$-Ph-BPDA- $\left.\mathrm{C}_{10}\right)$ polymer can be processed in the same fashion, although no strain-induced crystallization takes place. Nevertheless, the drawn amorphous poly $\left(p-P_{-}\right.$-BPDA- $\left.{ }_{10}\right)$ tapes still exhibit a tensile modulus of $3.65 \mathrm{GPa}$ and a stress at break of $362 \mathrm{MPa}$. Moreover, the poly( $p$-Ph-BPDA$\mathrm{C}_{10}$ ) tapes reverted back to their original shape upon heating beyond their glass transition temperature, allowing for their deformation without significant loss of properties, a feature similar to that of a shape-memory material. Overall, these findings demonstrate that the new semi-crystalline polymers based on the bispyrrolidone dicarboxylic acids from itaconic acid are displaying promising mechanical performance, in particular when processed into drawn tapes.

\section{Conflicts of interest}

There are no conflicts to declare.

\section{Acknowledgements}

This work was performed under the framework of Chemelot InSciTe and is supported by contributions from the European Regional Development Fund (ERDF) within the framework of OP-Zuid and with contributions from the province of Brabant and Limburg and the Dutch Ministry of Economy. 


\section{References}

1. Noordzij, G. J. et al. The aza-Michael reaction: towards semi-crystalline polymers from renewable itaconic acid and diamines. Polym. Chem. 10, 4049-4058 (2019).

2. Qi, P., Chen, H.-L., Nguyen, H. T. H., Lin, C.-C. \& Miller, S. A. Synthesis of biorenewable and water-degradable polylactam esters from itaconic acid. Green Chem. 18, 4170-4175 (2016).

3. Wang, R. et al. One-pot synthesis of biodegradable and linear poly(ester amide)s based on renewable resources. J. Appl. Polym. Sci. 133, 43446 (2016).

4. Roy, M., Noordzij, G. J., van den Boomen, Y., Rastogi, S. \& Wilsens, C. H. R. M. Renewable (Bis)pyrrolidone Based Monomers as Components for Thermally Curable and Enzymatically Depolymerizable 2-Oxazoline Thermoset Resins. ACS Sustain. Chem. Eng. 6, 5053-5066 (2018).

5. Ayadi, F., Mamzed, S., Portella, C. \& Dole, P. Synthesis of bis(pyrrolidone-4-carboxylic acid)-based polyamides derived from renewable itaconic acid-application as a compatibilizer in biopolymer blends. Polym. J. 45, 766-774 (2013).

6. Wang, Z. et al. Synthesis of fully bio-based polyamides with tunable properties by employing itaconic acid. Polymer (Guildf). 55, 4846-4856 (2014).

7. Meijer, H. E. H. \& Govaert, L. E. Mechanical performance of polymer systems: The relation between structure and properties. Prog. Polym. Sci. 30, 915-938 (2005). 


\section{Summary}

Apart from the many beneficiary product properties plastic materials have given us, the vast majority of these plastics are made from non-renewable fossil resources such as crude oil. The use of fossil fuel resources is under scrutiny due to the inherent rise in anthropogenic $\mathrm{CO}_{2}$ in the atmosphere, which is linked to the global warming and changing weather patterns we are currently facing. Therefore, a transition in the chemical industry's feedstock from nonrenewable fossil fuel resources towards renewable resources is a viable pathway to reduce the anthropogenic $\mathrm{CO}_{2}$ emissions in the future. Currently, roughly $15 \%$ of the extracted fossil fuel resources are being used in the production of chemicals and plastics. Therefore, transitioning to a renewable feedstock for the chemical and plastic industry can have a large impact on reducing anthropogenic $\mathrm{CO}_{2}$ emissions. Since plastics are here to stay - which is a good thing- many research groups focusses on how renewable resources can give us new renewable plastics with the desired beneficiary product properties.

At present lignocellulosic biomass, obtained from secondary biomass resources such as corn stovers and switch grass, is annually harvested at sufficient scales to provide raw materials for the chemical industry. The structural components of plants in this lignocellulosic biomass is a mixture comprising of cellulose, hemi-cellulose and lignin. The complexity of this mixture is due to different chemical structures of the components, and thereby inhibit direct drop-in replacement in the existing petrochemical plants. Therefore, worldwide enormous efforts are being made to be able to utilize these biomass "waste" streams, partially via thermal and/or chemical depolymerization into the chemical building blocks of these plant components. These biobased chemical building blocks can subsequently be (bio)chemically modified into completely new chemicals and building blocks. The research described in this thesis encompasses the use of two such potential building blocks obtainable from hemi-cellulosic biomass: 1,3-cyclopentanediol (chapters 1-3), and 1,3-cyclopentanediamine (chapters 4-6). The new building blocks are used to synthesize novel biobased polymers, with the goal to achieve beneficial physical and mechanical properties required for high performance polymer applications.

This PhD research was part of Chemelot InSciTe's HORIZONTAL research program, and performed in the laboratories of the Aachen-Maastricht Institute for Biobased Materials (AMIBM). Considering the functionality of the monomers (a diol and a diamine) and the focus of the AMIBM research group on high performance engineering plastics, the research focus is set out to prepare various polycondensates with these monomers. The term high-performance revolves around making materials for low volume applications, e.g. mechanical parts, where key parameters are semi-crystallinity and the related mechanical performance (preferably strong and ductile). An important factor in choice for engineering plastics rather than bulk plastics for e.g. packaging is the cost 
price: the cost of development for a new monomer and the related novel polymers will be too high to compete to "classical" bulk plastics such as PE, PP, PS and PVC.

Considering the limited availability of both 1,3-cyclopentanediol and 1,3-cyclopentanediamine as starting material the project was set out to start with the development of a small-scale polymerization and analysis tool (chapter 1). A 57-parallel thin-film polycondensation reactor was successfully developed with which polymerizations on ca. $10 \mathrm{mg}$ scale could be performed. Due to high rates of evaporation of monomers in thin-film conditions first the various monomers are synthesized into trimer prepolyesters. These prepolyesters are found to be highly efficient for high-throughput screening in $10 \mathrm{mg}$ scale, where influence of catalyst, temperature, time, and co-monomer is readily studied. The small-scale polymerization is readily combined with analysis techniques which require small amounts of material such as TGA, DSC, GPC, MALDI-ToF-MS, FTIR, and NMR. However, mechanical performance such as tensile testing, DMTA and rheology of these polymers could not be studied since more material is required (in the order of grams).

The structure-property relationship between the chemical nature of the cyclopentane ring and the polymer properties is readily studied for the polymers obtained from the smallscale polymerization setup. The structure-property relationship is compared to industrially relevant cyclic monomers such as 1,4-cyclohexanediol and diamine, and 1,4-cyclohexanedimethanol, which is a common trend throughout this entire thesis. A comparative study (chapter 1) for thermal transition temperatures show that polyesters having cyclopentanediol exhibit lower $T_{\mathrm{g}}$ and $T_{\mathrm{m}}$ than cyclohexanediol, but a similar $T_{\mathrm{g}}$ and $T \mathrm{~m}$ to cyclohexanedimethanol, albeit with a hampered crystallinity. The lowering in thermal properties and crystallinity is likely caused by the less stable half-boat conformation of cyclopentane compared to the stable boat-conformation of cyclohexane structures. Furthermore, 1,3-cyclopentanediol display the characteristic cis/trans behavior where polymers with mainly the trans isomer are semi-crystalline in nature, and those with increasing cis-isomer crystallization is hampered, and eventually lost while reaching concentrations of $40 \%$ cis-isomer.

A limitation for the use of 1,3-cyclopentanediol in high-temperature meltpolycondensation is that both isomers are found to be inherently thermally instable (chapter 2). Trans-1,3-cyclopentanediol can be used up to ca. $220{ }^{\circ} \mathrm{C}$, but at higher temperatures dehydration of the end-groups takes place. This dehydration gives rise to unsaturated bonds which during prolonged heating causes various side-reactions leading to o.a. cross-linking. Cis-1,3-cyclopentanediol is found to be highly unstable to elevated temperatures: Already at $180^{\circ} \mathrm{C}$ severe polymer degradation takes place, and preliminary mechanistic investigations suggest this happens via intramolecular hydrogen-bonding, which leads to the complete loss of the cyclopentanediol moiety in the polymer, leaving the acid end groups. These acid end groups in turn catalyze the dehydration and chain-scission reactions, thus effectively autocatalyzing the polymer degradation. These temperature limitations indicate that 1,3-cyclopentanediol cannot be 
used directly in the polycondensation with the industrially relevant terephthalic acid or 2,5-furandicarboxylic acid because of the too high melting temperature. This effectively means that cyclopentanediol is not capable of competing with cyclohexanediol and cyclohexanedimethanol for the envisioned engineering plastics.

The potential of 1,3-cyclopentanediol as comonomer in the production of biobased polymer PEF is studied for the effect on $T_{\mathrm{g}}, T_{\mathrm{m}}$ and gas-barrier properties (chapter 3 ). Industrially relevant cyclic diols in the co-polymerization of PEF typically give a rise in $T_{\mathrm{g}}$ due to the rigidity of the ring-structure, but also hamper crystallization. For copolymerizing 1,3-cyclopentanediol it is found that the $T_{\mathrm{g}}$ is actually lowered while also crystallinity is hampered, confirming previous findings that the rigidity of cyclopentanediol is inferior to cyclohexane counterpart. Preliminary oxygen permeability studies on polymeric films of the co-polymers indicate that there is a lowering of overall barrier properties, which is typical for the co-polymerization of cyclic diols in PEF. Normally with the copolymerization of industrially relevant cyclic diols there is a trade-off between increase in $T_{\mathrm{g}}$ and slight decrease in barrier properties. However our findings indicate that 1,3-cyclopentanediol lowers both, and is therefore deemed as not suitable for this kind of applications.

Preliminary results of direct polycondensations with 1,3-cyclopentanediamine indicate similar results to polyesters based on 1,3-cyclopentanediol: The thermal properties are similar to the 1,4-cyclohexanedimethylamine counterpart, albeit with hampered crystallization. Though these polyamides are difficult to characterize due to solubility issues, and limited thermal stability, a different approach was taken. The diamines are transformed into dicarboxylic acids by a benign reaction with two equivalents of renewable itaconic acid (chapter 5). The resulting 1,3-cyclopentanediamine based bis(pyrrolidone carboxylic acid) (BPDA) is thermally stable and is readily polymerized with aliphatic diols to generate polyesters. The resulting polyesters are amorphous in nature, with a $T_{\mathrm{g}}$ around $30^{\circ} \mathrm{C}$ and readily absorb water. The benign transformation of instable diamines to thermally stable dicarboxylic acid intermediate is highly promising, therefore a mini-review on this topic was written and published (chapter 4).

Although the BPDA-based polyesters based on 1,3-cyclopentanediamines do not lead to advantageous polymeric properties, BPDA-based polyesters based on trans-1,4cyclohexanediamine and para-phenylenediamine are found to be highly promising as polymers for high-performance applications (chapter 5). The BPDA-based polyesters based on trans-1,4-cyclohexanediamine or para-phenylenediamine polymers exhibit semi-crystalline behavior. The semi-crystallinity behavior, with a moderate $T_{\mathrm{g}}\left(30-45^{\circ} \mathrm{C}\right)$ and high $T_{\mathrm{m}}\left(170-210^{\circ} \mathrm{C}\right)$, and high crystallization rates have never been described before for this class of polyesters. These polymers have a high (potential) biomass content $(\sim 75-80 \%)$, and are readily synthesized via standard polycondensation techniques. Preliminary mechanical performance and crystallization is studied to assess the potential use of this new class of semi-crystalline polymers (chapter 6). For both polymers, given that they have sufficiently high molecular weight, are found to be ductile 
at room temperature when in the amorphous phase, and can be cold-drawn up to $500 \%$. When isotropically crystallized the polymer materials displayed brittle failure with a low modulus. However, annealed drawn tapes of the trans-cyclohexane based polymer lead to oriented semi-crystalline and tough fibers with a tensile modulus ranging from $710 \mathrm{MPa}$ to $3.1 \mathrm{GPa}$ and stress to break ranging from $94 \mathrm{MPa}$ to $138 \mathrm{MPa}$. The polymer based on para-phenylenediamine can be processed in the same fashion, although no straininduced crystallization takes place during the cold-drawing. Nevertheless, these colddrawn amorphous tapes still exhibit a tensile modulus of $3.65 \mathrm{GPa}$ and a stress at break of $362 \mathrm{MPa}$. Moreover, these drawn tapes revert to their original shape upon heating beyond their glass transition temperature, allowing for their deformation without significant loss of properties, a feature similar to that of a shape-memory material. 


\section{Valorization addendum}

The common goal in this $\mathrm{PhD}$ research was to develop high performance polymer materials from new biobased building blocks. High performance polymers are a group of polymers which have good mechanical, thermal, and chemical properties, which are retained under various "working" conditions: The performance should be good and stable during e.g. mechanical loading, change in temperature or medium. Currently such high performance properties are often gained by (co-)polymerization of rigid building blocks such as cycloaliphatic 1,4-cyclohexanediol and 1,4-cyclohexanedimethanol. These building blocks are typically used as (co-)monomer to increase, amongst others, the thermal stability and melt-strength of polymers. A well known example is the copolymerization of 1,4-cyclohexanedimethanol in PET which strongly enhance the toughness. The rigid cyclohexane-ring gives rise to these beneficial properties, however currently most of such rigid (co-)monomers used are sourced from petrochemical. Hence, this research is set out for the development of (novel) renewable cycloaliphatic monomers, which can be used as a renewable alternative.

As already described in detail in chapters 1-3 in this thesis, in our hands polyesters based on the potentially renewable building block 1,3-cyclopentanediol could not be used in performance polymers. This is mainly related to the inherent thermal instability of the hydroxyl end-groups limiting the temperature for melt-polycondensation. Also the obtained polyesters with 1,3-cyclopentanediol were not stable, which resulted in degradation upon melt, thus effectively preventing melt-processing. Further use of 1,3cyclopentanediol as co-monomer proved challenging to synthesize high molecular weight polyesters, and did not result in result in beneficial properties on e.g. the glass transition temperature $\left(T_{\mathrm{g}}\right)$.

In contrast, the developed cyclic monomers based on the cascade aza-Michael additioncyclization between itaconic acid and various diamines proved to be highly interesting (Chapter 4, this thesis). The use of cyclic diamines such as trans-1,4cyclohexanediamine $(t-\mathrm{CH})$ and para-phenylenediamine $(p-\mathrm{Ph})$ yielded tri-cyclic bispyrrolidone monomers: $t$-CH-BPDA and $p$-Ph-BPDA (Chapter 5 , this thesis). Polyesters based on these BPDAs with 1,10-dodecanediol have good thermal properties, likely originating from the rigid tri-cyclic BPDA moieties. Especially the drawn tapes of poly([t$\left.\mathrm{CH}-\mathrm{BPDA}]-\mathrm{C}_{10}\right)$ and poly $\left([p-\mathrm{Ph}-\mathrm{BPDA}]-\mathrm{C}_{10}\right)$ show very promising mechanical properties (Chapter 6 this thesis). Indeed, this chapter beautifully shows how the polymer properties are determined by 3 factors: chemistry, molecular weight, and processing. With these 3 parameters right we obtained brittle to ductile transition (molecular weight), brittle to strong crystals (processing), increase in $T_{\mathrm{g}}$ (processing), high melting (chemistry) and water resistant (chemistry) properties for a new class of polyester, which is $\sim 80 \%$ biobased. Even though the development of these polymers is still in the very beginning, 
we can think of two possible target applications: strong fibers, and heat-shrink polymers tunable for the shrink temperature (e.g. body temperature) with high water stability and biocompatibility.

\section{$\operatorname{Poly}\left([t-C H-B P D A]-C_{10}\right)$}

The good mechanical properties of drawn tapes of poly $\left([t-\mathrm{CH}-\mathrm{BPDA}]-\mathrm{C}_{10}\right)$ with oriented crystals can be at the basis of further development of these fibers. Current drawing experiments have been performed at room temperature (r.t.), with a separate annealing step at $60^{\circ} \mathrm{C}$. Annealing in the drawn state lead to the formation of the oriented crystals, giving an increase in mechanical properties, whilst remaining ductile. This batch-wise process is undesirable for larger scale processing, hence a logical next step would be to study the crystallization rate during drawing. This can be performed by in situ XRD measurements (e.g. at synchrotron facilities at Grenoble or Alba), where the effect of draw rate at various temperatures can be measured on the crystallization. The results of these measurements can then function as a base for the production of fibers from a meltextrusion spinning line as present within the AMIBM laboratories (Figure 1). During meltextrusion the molten polymer can be pushed through a spin-plate with small nozzles, from which the polymer can be pulled into small fibers. The fibers pass through an air quench step to produced amorphous fibers, similar to how the polymer films described in Chapter 6 are quenched. Subsequently these quenched amorphous fibers can be drawn by the rotating godets at various temperatures, to obtain the desired orientation and crystallization.

There is a wide range of applications for polymeric fibers, including producing yarns for various textile products. The direct applications for fibers (or tapes) from our developed polyester is still uncertain, as first several key parameters need to be studied. These include the temperature stability, (effect of) moisture absorbance, chemical stability, biocompatibility and biodegradation/biotoxicity. Though we estimate the temperature stability is high ( $T_{\mathrm{m}}$ of oriented crystals is around $180^{\circ} \mathrm{C}$ ), moisture absorbance is minimal, and therefor has a high stability in aqueous environment (stable against hydrolysis at r.t.). In terms of biocompatibility preliminary cytotoxicity tests have been performed on amorphous isotropic films of both developed polyesters in collaboration with the MERLN institute (Rebeca Rivero, dr. Matt Baker). The polymer films were tested with a Presto Blue cell viability assay via cell-contact measurements on L929 cells. L929 cells were seeded in 96-well plates, and after one day a piece of the polymer was placed in contact with the cells and incubated for 24 and $48 \mathrm{~h}$ at $37^{\circ} \mathrm{C}$. After those times the polymer piece was removed, and the Presto Blue solution was added to the wells and further incubated for $1 \mathrm{~h}$ at $37^{\circ} \mathrm{C}$. The fluorescent intensity between the control (polystyrene), poly $([p-$ 
Ph-BPDA $\left.]-\mathrm{C}_{10}\right)$ (polymer 1) and poly $\left(\left[t-\mathrm{CH}-\mathrm{BPDA}-\mathrm{C}_{10}\right)\right.$ (polymer 2) are not significant after $48 \mathrm{~h}$, indicating absence of toxicity (Figure 2).

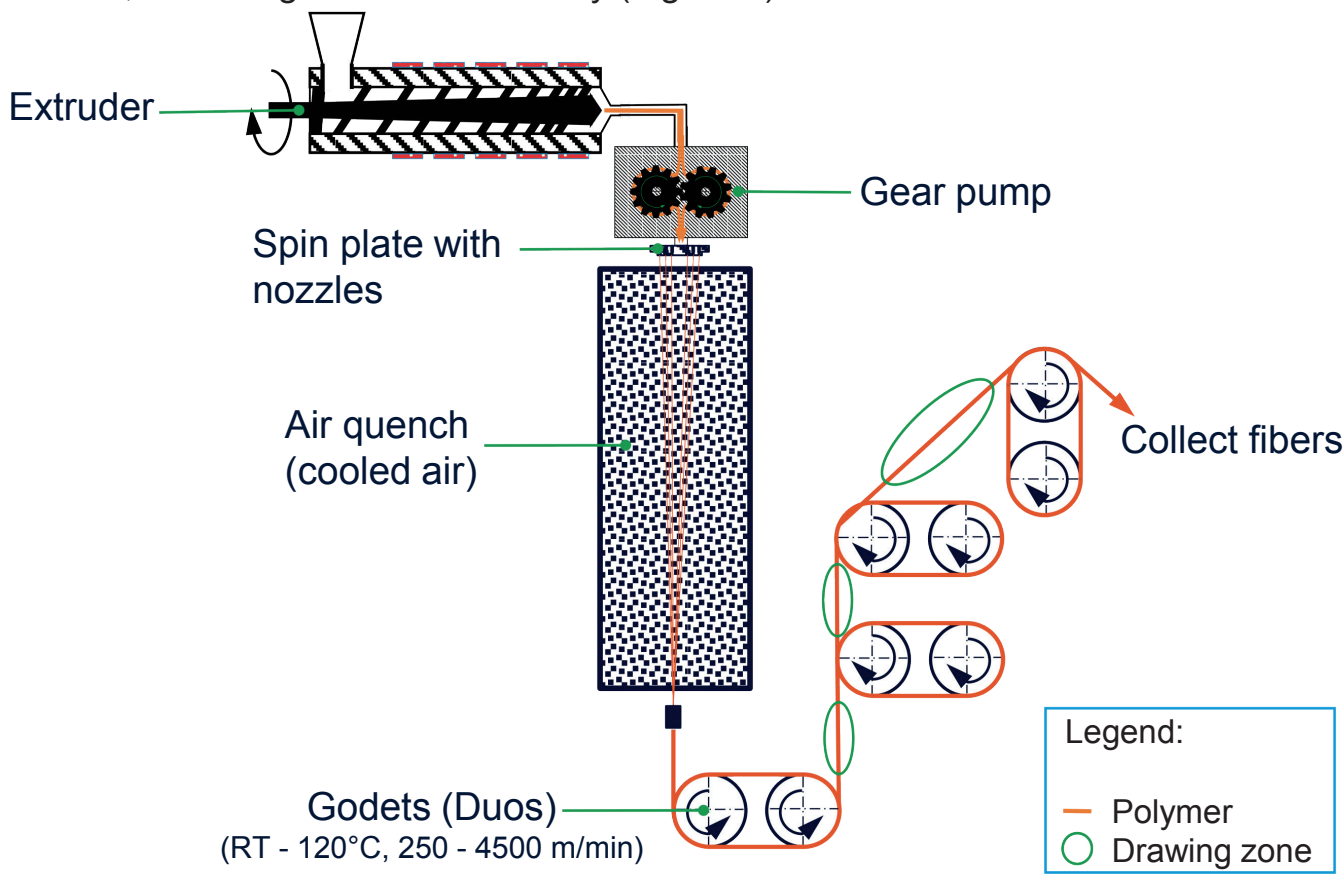

Figure1. Schematic overview of melt-extrusion line to produce oriented polymeric fibers as present in the laboratories of AMIBM (image courtesy of Stefan Hermanns). Melt-extruded polymer if pumped through a spin plate with nozzles, from which polymer fibers are drawn. The fibers are first quenched by cooled air, and subsequent drawing steps are realized on multiple rotating godets, which can rotate at different speeds and temperatures to obtain the preferred draw ratio and crystallization.

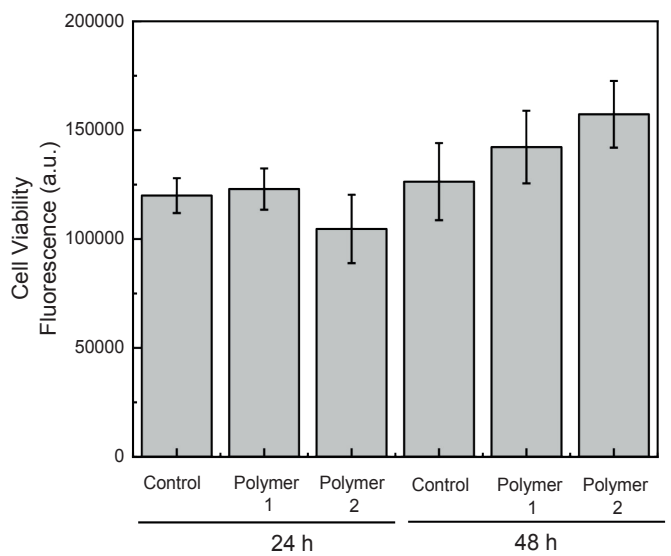

Figure 2. Presto Blue cell viability assay via cell-contact measurements on L929 cells for our developed polyesters poly $\left([p-P h-B P D A]-C_{10}\right)$ (polymer 1$)$ and poly $\left([t-C H-B P D A]-C_{10}\right)$ (polymer 2$)$, controlled against polystyrene. After $48 \mathrm{~h}$ there is no significant difference between our polymers and the control, indicating absence of toxicity for cell-contact. 
Though these (bio)toxicity experiments are very preliminary, it is at least good to know that cells do not immediately die upon contact with our developed polyesters. A next step would be to perform controlled biodegradation (e.g. in enzymatic solution) or hydrolysis (e.g. in hot water), and study the biotoxicity of the obtained products. If biotoxicity indeed proofs to be low it is also important to study whether cells or not can grow on the polyester surface. If so this can open up interesting possibilities for in vivo applications for our developed polyesters.

\section{Poly([p-Ph-BPDA]-C 10$)$}

In contrast to the poly $\left([t-\mathrm{CH}-\mathrm{BPDA}]-\mathrm{C}_{10}\right)$ drawn tapes, the drawn tapes of poly $([\mathrm{p}-\mathrm{Ph}-$ BPDA]-C ${ }_{10}$ ) proved much harder to crystallize in the drawn state. Rather, we observed the rapid shape memory behavior as soon as the oriented tapes are heated to above their $T_{g}$. Such shape memory behavior is typical for polymers which are kinetically trapped in the stretched state, and applications are commonly found in heat shrink foils. Also, as discussed in Chapter 6 , the $T_{\mathrm{g}}$ changes as a function of the drawing ratio, and as described in Chapter 5 , the $T_{\mathrm{g}}$ also changes depending on the diol spacer length. It would be interesting if the temperature trigger for the shape memory behavior around body temperature $\left(38-40{ }^{\circ} \mathrm{C}\right)$. This can be achieved by increasing to diol spacer length to e.g. $\mathrm{C}_{12}$ diol, after which the draw ratio will determine the final trigger temperature. If the biocompatibility of this polymer is indeed good, there are some interesting in vivo applications for heat shrink materials which shrink at body temperature. For example, there are applications where an expanded tubular polymer is placed over a (repaired) blood vessel, which after a mild temperature treatment contracts and forms a fixed bandage over the blood vessel. One important thing to investigate is need for mechanical performance of these heat shrink foils during application. As we have seen in Chapter 6 , the drawn state of poly $\left([t-\mathrm{CH}-\mathrm{BPDA}]-\mathrm{C}_{10}\right)$ is a tough material, but the amorphous isotropic state (as it will be after heat shrinkage) is soft and readily deforms.

\section{Scaling up}

Scaling up and marketability is highly dependent on pricing and availability of the used monomers. The main bottle-neck is trans-1,4-cyclohexanediamine $(t-\mathrm{CH})$, which is not produced as commodity chemical, and is highly expensive: currently ranging around $€ 1000 /$ kilo at commercial suppliers. However, its precursor, and also one of our monomers, is para-phenylenediamine ( $p$-Ph) which is produced cheaply on industrial scale. At this moment $p-\mathrm{Ph}$ is not available from biomass, and thereby limiting the biobased content in our developed polyesters to about $80 \%$ (atom-based). An interesting research project to set up would be to develop a biobased route towards $p$-Ph. This could be initiated with a research collaboration with the start-up BioBTX, which could produce the required raw material from biomass. In turn, this can be used as a drop-in in existing chemical plants to produce $p$-Ph. Further hydrogenation towards $t-\mathrm{CH}$ and subsequent separation of the isomers is currently performed batchwise in low quantities. However, 
with a potential market pull from our developed polymers it can be worthwhile to develop a route and perform a cost-optimization to estimate the costs of the final polymer if these routes are developed.

\section{End of life?}

A very important factor in the ecological footprint of polymers is not only the sourcing, but also the end-of-life options. Currently, a lot of money is invested to enhance the wastemanagement and recycling technologies to obtain clean waste-streams of plastic ready to be reprocessed. The recycling efficiency is determined by two main parameters: the amount of material that can be recycled (\% of total volume), and purity of the recycled fraction. Unfortunately, at the current technology level, introducing new (biobased) plastics to the market will inherently lead to an even more complex waste stream of plastic materials to sort, potentially negatively influencing the recycling efficiency. However, it is not very relevant to highlight this too much at this stage, as the total market volume at this moment for biobased plastics is hovering around $1 \%$. Furthermore, recyclers use near-infrared (NIR) technologies to identify and separate the different plastics in the recycling stream. The newly developed biobased plastics have an unique NIR signal, and will easily be removed from the waste streams, avoiding pollution of the waste streams of e.g. PET, PE, PP. More importantly, the majority of the plastics produced today do not follow the "design to recycle" principle, which is visible in the large variety of additives and multilayer filaments, effectively reducing the recycling efficiency by making "inferior mixed granulates". So if the potential increase of complexity of the plastic waste streams would be an argument against biobased plastics on the market, then the same holds true for the production of mixed-plastic (packaging) on the market which are not designed to recycle.

Alternatively, we can learn from the current end-of-life options for the biobased polymer PLA. Even though PLA can excellently be recycled, the current production and market volume of PLA is so low that installing a separate waste-stream at plastic recyclers is not worth the investment. Rather, many PLA products are sold and labelled as industrial compostable, which means that consumers can dispose of their PLA products in the green bin at home. Although from an environmental point of view recycling is of course much better than composting, at the current production scales industrial composting is a viable alternative. The use, and end-of-life, of PLA can function as an excellent example for the development of other high-performance biobased polymers. With this in mind it would be good, if larger scale production of our developed polyesters will happen, to study whether industrial composting is a viable end-of-life solution. Obviously when production volumes further increase direct recycling routes should be developed for all new (biobased) polymers, perhaps via new deposit-schemes as currently exists for some PET bottles, or by installing extra NIR-separating lines at the plastic recyclers. 


\section{Dankwoord}

And so my adventure to Chemelot (no not Camelot) and Maastricht has come to an end!

Allereerst wil ik mijn begeleiders Karel en Sanjay bedanken. Sanjay, thank you for "taking me in" into the brand new research group that was at the time being set-up at UM. A fresh start for me was exactly what I needed, and I would say it worked out great in this new group! So thanks for the opportunity, and good luck with your future endeavors. Karel, chef, enorm bedankt voor alles! We zijn van directe collega's naar "student/begeleider" rol gegaan, maar eigenlijk is daar nooit iets van te merken geweest. Het was ontzettend fijn om met jou samen te werken, ook al moest ik jou de scheikunde af en toe uitleggen, jij hebt mij enorm goed geleerd om de vertaalslag te maken van labresultaat naar publicatie! Dat was het stuk leercurve wat ik enorm nodig had, en gaandeweg heb ik zowaar ook nog het e.e.a. geleerd van jouw vak; polymeer engineering! Fijn dat wij altijd als gelijke de gedachtewisseling aan konden gaan, dat, en jouw rust, siert je enorm!

Of course finishing up the PhD degree doesn't go without the active participation of the reading committee and corona-members. So a special thank you to Prof Maarten Honing, Dr. Matt Baker, Prof Rint Sijbesma and Prof Stefan Bon for their time in assessing my $\mathrm{PhD}$ thesis and taking part in the corona. Rint, lang geleden heb ik in jouw groep aan de TU/e stage gelopen, en ook al had ik destijds besloten mijn PhD onderzoek elders voort te zetten ben ik blij dat je op deze manier toch nog betrokken bent bij mijn promotie.

En dan iemand die eigenlijk als eerste had moeten staan, maar soms loopt het anders dan je van te voren verwacht. Bart, bij deze nogmaals enorm bedankt voor jouw mateloze inzet bij SPM, en bij mijn oorspronkelijke PhD project aan de TU/e. Het blijft natuurlijk enorm jammer hoe de dingen gelopen zijn, maar later werd het ook weer duidelijk; uiteindelijk vinden we allemaal onze weg weer. En ik ben enorm blij dat ik je op deze manier toch nog kan bedanken, bij jou ben ik de weg naar biobased polymeerchemie begonnen, en daar wil ik voorlopig niet mee stoppen!

I would like to thank my direct and indirect colleagues from Chemelot InSciTe for the collaboration throughout these 4 years. It was an interesting experience to start such a massive project from 5 different points at the same time, and discuss results from so many different scientific angles. Thanks Carin, Christian, Myrto and Vladan for the collaborative research. Thanks Henk, Natascha, Paul, PQ and Solomon for steering the projects and meetings. And thanks Annabel, Emiel and Ingrid for the administrative, organizational and steering force at Chemelot InSciTe.

Bij het opzetten van een nieuwe vakgroep hoort natuurlijk enorm veel inzet van allerlei partijen. Vooral Yvonne wil ik bedanken, niet alleen voor jouw voortdurende inzet, maar 
ook voor alle gezellige en leerzame gesprekken die we gehad hebben. Veel succes met jouw onderzoeksgroep en aanstaande promotie tot professor! Natuurlijk ook veel dank naar Richard, Wouter en later Ermo om alles in de goede banen te sturen. For all the early PhD's; Ola, Marie, Varun, Christian, Vahid, Gijs, Manta, Andrea, Monika, Anne and Martien, thanks for the good times and making sure the institute could grow! There've been many other colleagues in centercourt which have made it a great time with lovely atmosphere to work there; thanks Carlos, Dario, Feiji, Huaqin, Ramiro, Jules, Lawrence, Luciënne, Milo, Mitch, Mohan, Pouya and Renato! Paul, Jurrie, Nils bedankt voor jullie inzet en technische ondersteuning, Arjen, Bea, Nico en Will, bedankt voor het labonderhoud en jullie altijd goede humeur! Marcelle bedankt voor de goede administratieve bijdrage. Nils, chapeau jongen, het was super om je te leren kennen en met je samen te werken, ik kom nog wel een keertje terug om met je Lego te spelen. For all the others in AMIBM, thank you for the efforts you've put in AMIBM, it has been a pleasure seeing the institute grow, and slowly mature. I wish you, and AMIBM, a pleasant future!

Also during the years l've had the pleasure to work with many students, in practical courses, project groups but moreover also with supervision of the internship/graduation thesis research. Thank you (in order of appearance) Joshua, Yara, Catherine, Daan, Natasja, Vincent and Sanne for your trust and time. Many of you have found your way back on one of the publications!

Lieve familie, het was natuurlijk een kleine schok dat ik naar het o zo diepe zuiden ging verhuizen! Weliswaar hebben wij elkaar minder gezien dan gebruikelijk, maar de steun en verbondenheid is over de jaren alleen maar toegenomen. Enorm bedankt daarvoor! Anne, zussie, gelukkig hebben wij nog zeer regelmatig samen kunnen borrelen, stappen en dansen de afgelopen jaren. Bedankt voor de goede en gezellige avonden en nachten, en dat er nog maar vele mogen volgen! Pap, ik heb het enorm fijn gevonden hoeveel wij elkaar zijn blijven zien de afgelopen jaren. Tijdens vele wandelingen hebben wij altijd alles wat met het leven komt kunnen bespreken en delen, bedankt voor deze onvoorwaardelijke tijd en steun!

Lieve Uranymusvriendjes, ik ben natuurlijk weggaan van een ongelooflijk lekker en goed gevuld sociaal leven in Utrecht, voornamelijk dankzij jullie! Vooral onze "er was eens"groep, hoeveel wij wel niet gedeeld hebben! Bassie, Maaike, Martin, Tinus en Thijs: naar Maastricht gaan was natuurlijk een deur openen naar een ietwat ander sociaal leven, maar heerlijk hoeveel contact we nog steeds hebben. Thijs, wij zijn vooral in de laatste jaren enorm dicht naar elkaar gegroeid! Enorm bedankt voor de ontelbare deugden en ondeugden die we samen uitgespookt hebben afgelopen jaren, mooie tijd! En ook fijn dat jij, en nu met Diandra, mijn vaste logeeradresje in Utrecht is geworden, altijd een lekker ontbijtje! Martin en Inge, jullie ook bedankt voor de betrokkenheid, bezoekjes, en vele pannenkoekontbijtjes. Maaike, voor ons zat er nog een extra stuk afscheid bij voor mijn vertrek naar het zuiden, maar ik vind het heel fijn hoe wij nu weer met elkaar gaan. Bassie, bedankt voor de leuke vakanties, en we moeten snel maar onze roadtrip af 
maken! Voor de andere Uranymusvriendjes: ik had nooit verwacht dat we zoveel jaar na het afstuderen nog zoveel contact zouden hebben. Uiteraard heb ik wel het een en ander moeten missen, maar als we elkaar dan zo nu en dan nog eens tegenkomen is het altijd weer top zoals vanouds! Dus Anne, Atze, Claudia, Dina, Esther, Fabio, Feike, Kevin, IrisB, IrisH, Jan, Japse, Jasper, Jeroen, Jeroentje, Johanna, Kim, Lars, Loek, Maaike, Maarten, Malou, Marlijn, Nol, Paul, Rianne, Rienko, Ronne, Sander, Suus, TimB, TimF, en Tom bedankt en tot snel! ${ }^{1}$

En bij een nieuwe stad hoort natuurlijk ook weer een nieuw leven, en die is rijk gevuld geweest! Het begon met vrijwilligerswerk bij het asiel, een superleuke ervaring voor 1,5 jaar, vooral bedankt naar Liz en Brigitte! En ik heb er nog onbedoeld een kat aan overgehouden ook, dat had ik nooit gedacht! Social life continued with many international friends: Fabio, Frank, Gustavo, Leah, Luan, Sashi and especially Adrian, a big thanks for all the fun evenings filled with good conversations, dinners and drinks. Ook Anne, bedankt voor alle bezoekjes en gezellige spelletjesavonden! And then there is PhD Academy! It shouldn't be a big surprise that I quickly joined the social committee to organize more fun filled evenings! Thanks for the many good years and happy memories Bruna, Carey, Evelyn, Inès, Jan, Margaux, Mike, Martien, Tate and Timme! We shall toast again soon! Beth, sometimes it's crazy how fast a new friendship can form, but we managed! Thanks for the many crazy nights, fun parties and good times! People from BackForward and the bootcamp sessions, thanks for helping me recover from the Saturday-morning hangovers caused by the previously described people! Especially Germano, grazi buddy! It was great knowing you, both in doing sports as in having lengthy philosophical talks, l'll miss that!

En dan is er natuurlijk carnaval. Ik had als rasUtrechter nooit kunnen denken dat ik het ooit zou gaan vieren, danwel zo leuk zou gaan vinden, maar het tegendeel is bewezen! Frank en Marc, bedankt voor de jaarlijkse organisatie voor het bijeenbrengen van altijd weer een gezellige groep mensen, en voor mijn introductie aan vastelaovond in Mestreech! Thijs, elk jaar meerdere dagen meefeesten, genieten! En natuurlijk ook de andere vaste bezoekers Maaike en Rianne, altijd gezellig! Hopelijk volgend jaar weer, maar dan moeten we helaas wel een ander logeeradresje vinden.

Andrea, Goose and Varun, it's amazing how we went from random office mates to such close friends! Varun it was awesome to share a superb mansion in the middle of Maastricht with you, there where all the action was, and our house often functioned as living room or gathering place for our friends, thanks! The 4 of us really did so many things together, I'll fondly remember the dinners, drinks, roadtrips, holidays, beautiful beaches, angles, shots, tickling Varun, and screaming sessions while playing Mario Party. Thanks for all the good times, l'll miss you dearly. Manta, it took some time before I understood what all your different headshakes meant, but I'm very glad you came to work with us. From research and lab-buddies to life-long friends, I'm sure about that!

1) Vrij aangepast aan de hand van de thesis van Dr. Nol. 
Thanks for being there, and the crazy amounts of delicious(!) food you always cook. Good luck and all the best now that you are finally together with your hubby. Martien, last but definitely not least, we also went from colleagues to very dear friends. You to thanks for all the good times, good food, fun boardgames, and long talks with a drink or two. Thanks and all the best to you.

And remember, this is a goodbye, not a farewell!

Cheers,

Geert 


\section{Curriculum Vitae}

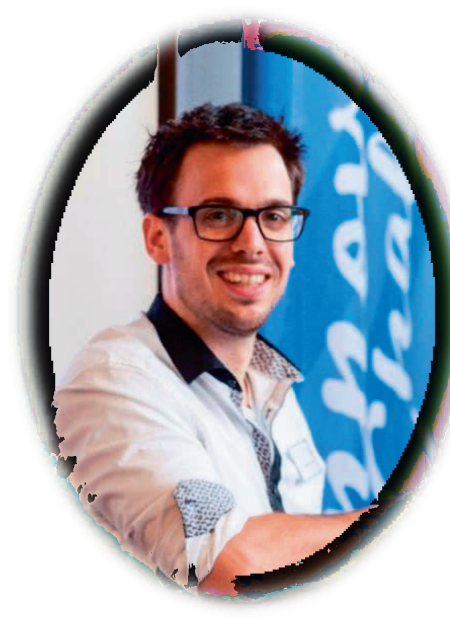

Geert Noordzij was born on July $10^{\text {th }}, 1987$ in Utrecht, The Netherlands. In 2010 Geert obtained his Bachelor of Applied Sciences from the Hogeschool Utrecht in the field of Chemistry - Research \& Development. During his bachelors he did an industrial internship at Henkel (Germany), and an academic bachelor thesis at the Institute for Chemical Research of Catalonia (ICIQ, Spain) in the group of Prof. dr. P.W.N.M. van Leeuwen. After a brief period of working in industry Geert did his Masters of Sciences from 2011-2013 at the University of Wageningen in the field of Molecular Life Sciences Physical Chemistry. During his masters he did his master thesis research with Dr. Tom Wennekes and Prof. dr. Han Zuilhof (Wageningen University), and his internship with Prof. dr. Rint Sijbesma (Eindhoven University of Technology). After obtaining his MsC in 2013 Geert started his PhD in the field of biobased polymer chemistry at the Eindhoven University of Technology under the supervision of Dr. Bart Noordover. Later in 2015 Geert started a new PhD research in the field of biobased polymer chemistry at the Maastricht University under the supervision of Dr. Karel Wilsens and Prof. Dr. Sanjay Rastogi. The work of the latter is described in this manuscript to obtain the degree of Doctor at the University of Maastricht.

On a personal and professional level Geert has a strong passion for sustainability, chemistry and plastics. With an academic background in biobased polymer chemistry, he has enjoyed working in diverse multinational teams performing research and development towards novel biobased polymers. He is looking forward to strengthen and use his expertise and enthusiasm to realize the needed transformations towards sustainable solutions, products, and processes for our society and our environment.

\section{Awards \& Prizes during PhD}

- 02-03 October 2018 - Best oral lecture price @ Chemelot InSciTe Annual meeting

- 20 April 2018 - Best poster price @ Applied Biobased Materials conference, Aachen Maastricht Institute for Biobased Materials (AMIBM)

- 09-10 April 2018 - Keltan Innovation Award, hosted by Arlanxeo, member of winning team from AMIBM (Maastricht University) 


\section{List of publications}

\section{Publications that have contributed to this thesis:}

Noordzij, G.J., Rastogi, S \& Wilsens, C.H.R.M. On the mechanical performance of semicrystalline bis(pyrrolidone) based polymers derived from itaconic acid. Manuscript in preparation

Noordzij, G.J., Rastogi, S., Adons, D., Peeters, R. \& Wilsens, C.H.R.M. Synthesis, thermal behavior and preliminary oxygen barrier properties of copolymers of 2,5-furandicarboxylic acid, 1,3-cyclopentanediol and ethylene glycol. Submitted

Noordzij, G.J. \& Wilsens, C.H.R.M. Cascade aza-Michael cyclizations towards renewable and multifunctional carboxylic acids for melt-polycondensation. Frontiers in Chemistry, 7, 729 (2019). Part of the research topic: Sustainable Polymers: Where Are We? Where Do We Go?

Noordzij, G.J., van den Boomen, Y.J.G., Gilbert, C., van Elk, D.J.P., Roy, M., Wilsens, C.H.R.M. \& Rastogi, S. The aza-Michael reaction: towards semi-crystalline polymers from renewable itaconic acid and diamines. RSC Pol. Chem., 10, 4049-4058 (2019).

Noordzij, G.J., Dietz, C.H.J.T., Leoné, N. Wilsens, C.H.R.M. \& Rastogi, S. Small-scale screening of novel biobased monomers: the curious case of 1,3-cyclopentanediol. RSC Adv., 8 , 39818-39828 (2018).

\section{Other publications by the author:}

Noordzij, G.J., Roy, M., Bos, N., Reinartz, V. \& Wilsens, C.H.R.M. Improving the hydrolysis rate of the renewable and biodegradable poly(hexamethylene sebacate) through copolymerization of a bispyrrolidone based dicarboxylic acid. MDPI Polymers, 11, 1654 (2019). In special issue: Sustainable Polymeric Materials from Renewable Resources.

Roy, M., Noordzij, G.J., van den Boomen, Y., Rastogi, S. \& Wilsens, C.H.R.M. Renewable (bis)pyrrolidone based monomers as components for thermally curable and enzymatically depolymerizable 2-oxazoline thermoset resins. ACS Sustain. Chem. Eng., 6, 5053-5066 (2018).

Pepels, M.P.F., Hermsen, I., Noordzij, G.J. \& Duchateau, R. Molecular structure-catalytic activity relationship in the ring-opening polymerization of (macro)lactones. ACS Macromolecules, 49, 3, 796-806 (2016). 


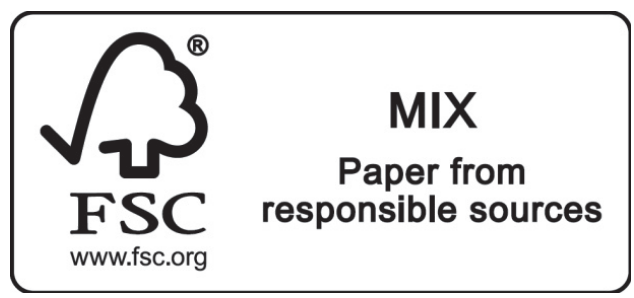




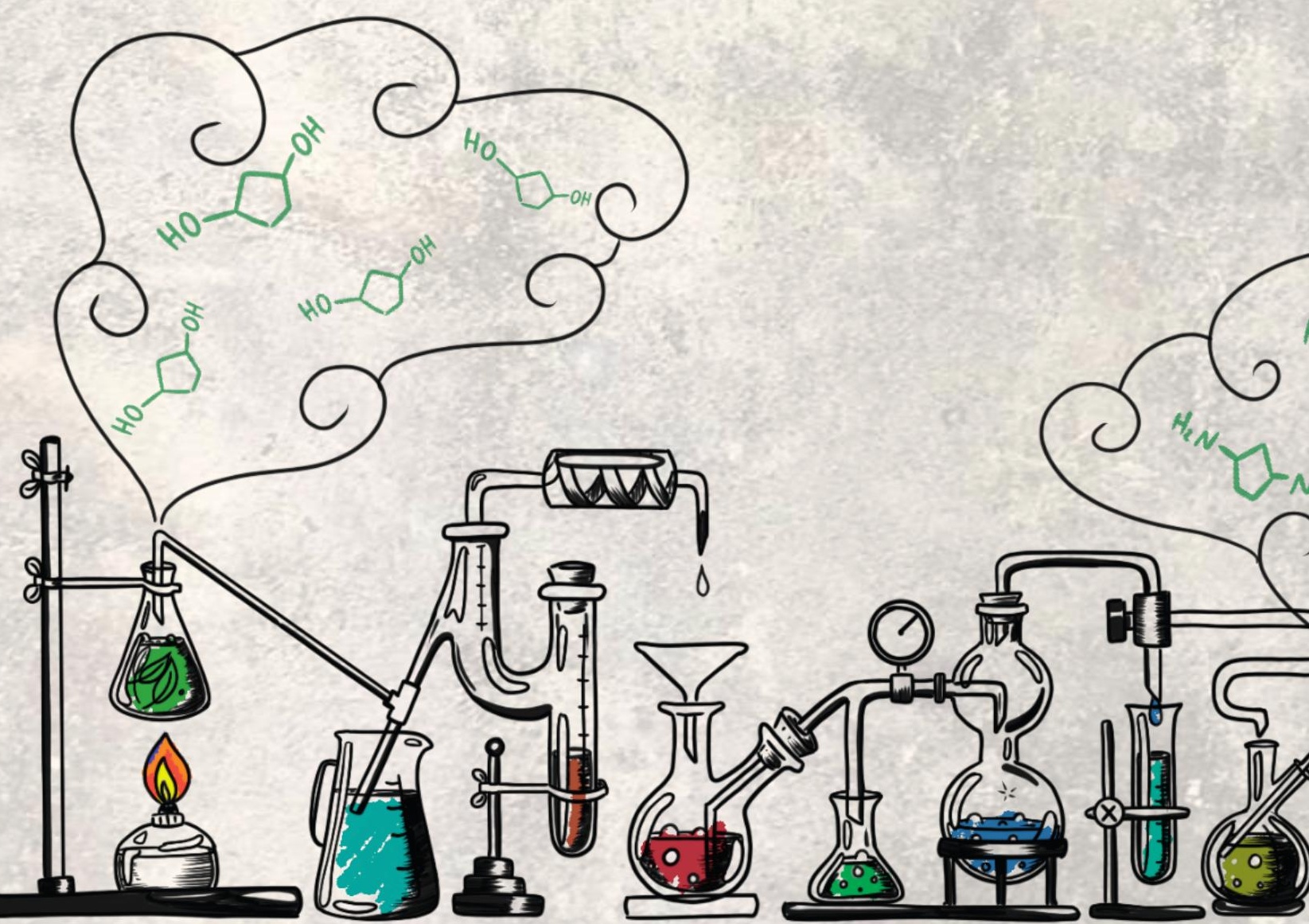

